\title{
Xiao Yingying
}

\section{Une subjectivité fluide}

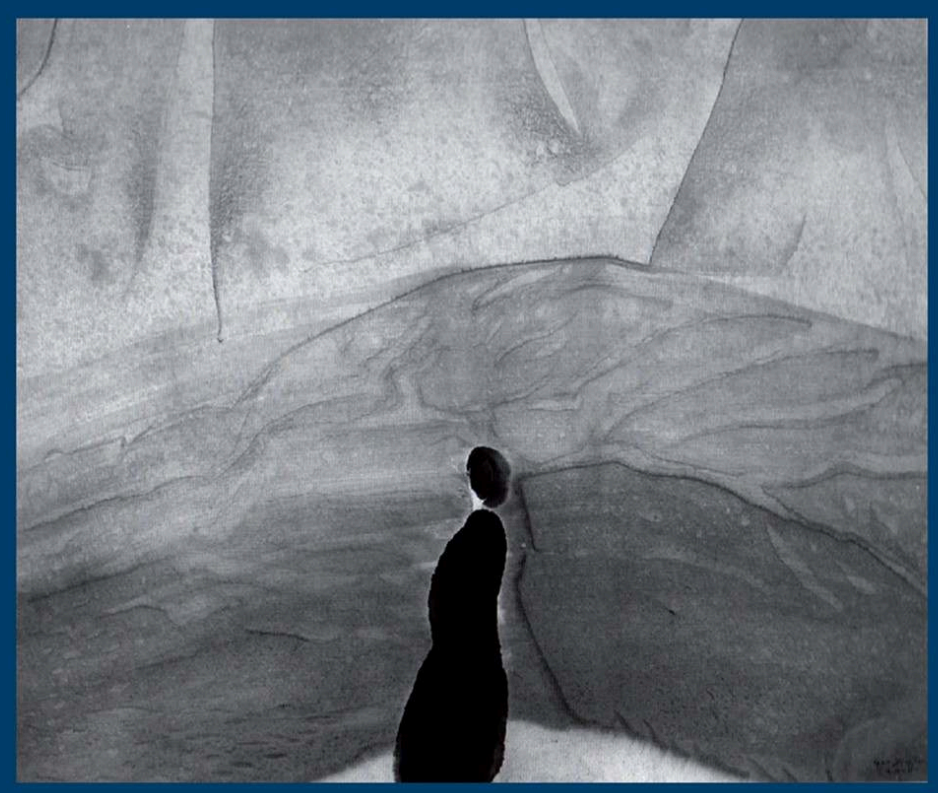

Préface de Léon Vandermeersch

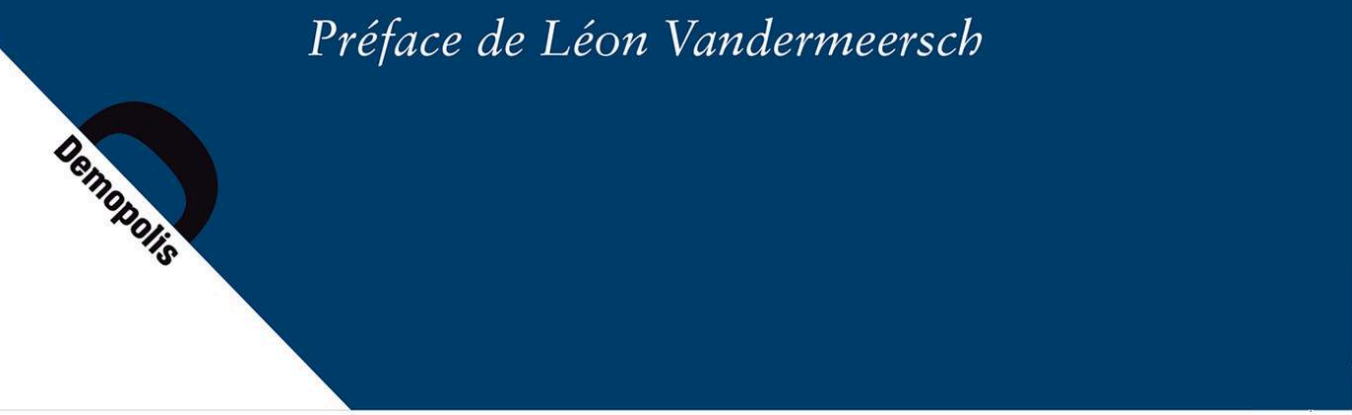




\section{Une subjectivité fluide}

Modernité et perception esthétique à travers les ouvrages de Gao Xingjian

\section{Yingying Xiao}

DOI : 10.4000/books.demopolis. 1518

Éditeur : Demopolis

Année d'édition : 2017

Date de mise en ligne : 18 janvier 2019

Collection : Quaero

ISBN électronique : 9782354571580

\section{Donertition Qbooks}

http://books.openedition.org

\section{Édition imprimée}

ISBN : 9782354571177

Nombre de pages : 313

\section{Référence électronique}

$\mathrm{XIAO}$, Yingying. Une subjectivité fluide: Modernité et perception esthétique à travers les ouvrages de Gao Xingjian. Nouvelle édition [en ligne]. Paris : Demopolis, 2017 (généré le 02 octobre 2020). Disponible sur Internet : <http://books.openedition.org/demopolis/1518>. ISBN : 9782354571580 . DOI : https:// doi.org/10.4000/books.demopolis.1518

(C) Demopolis, 2017

Conditions d'utilisation :

http://www.openedition.org/6540 


\section{UNE SUBJECTIVITÉ FLUIDE MODERNITÉ ET PERCEPTION ESTHÉTIQUE À TRAVERS LES OUVRAGES DE GAO XINGJIAN}




\section{«QUAERO »}

Collection dirigée par Jean-Christophe Tamisier

\section{Parmi nos parutions:}

Le Vietnam. Une histoire de transferts culturels, Hoai Huong Aubert-Nguyen et Michel Espagne (dir.)

Psychanalyser en langues. Intraduisibles et langue chinoise, Barbara Cassin et Françoise Gorog (dir.)

Illustration de couverture:

Perspective (2000), détail C Gao Xingjiang

CC Éditions Demopolis, 2017

67, rue Saint-Jacques

75005 Paris

www.demopolis.fr

ISBN : 978-2-35457-117-7 
XIAO YINGYING

\section{UNE SUBJECTIVITÉ FLUIDE MODERNITÉ ET PERCEPTION ESTHÉTIQUE À TRAVERS LES OUVRAGES DE GAO XINGJIAN}


Cet ouvrage a été publié avec le soutien du laboratoire d'excellence Transfers (programme Investissements d'avenir ANR-10-IDEX-0001-02 PSL* et ANR-10-LABX-0099) 


\section{Préface}

Dans la culture occidentale, la création littéraire est originellement fabrication de récits. Telle est la signification étymologique du grec epopoia (épopée en français), composé des mots poia ("fabrication », dérivé du verbe poiein, "faire ») et epos (" récit», dérivé de e-phonein, " émettre en parlant »). La littérature européenne commence en effet avec l'Illiade et l'Odyssée, les deux grandes épopées homériques. Ces deux épopées ont été créées en Grèce avant l'apparition de l'écriture, donc oralement, et leur oralité originelle n'a nullement été abolie par leur transformation en texte écrit. C'est que l'écriture qui a servi à opérer cette transformation était une écriture alphabétique, notant les phonèmes, c'est-à-dire l'oralité elle-même. Ainsi, dans la culture occidentale, où l'écriture est phonématique, il n’y a pas de différence de nature entre langage parlé et langage littéraire, mais différence seulement de degré de sophistication.

Dans la culture chinoise, il en va tout autrement. La création littéraire y est "sculpture de dragons dans l'esprit des graphies (文心 雕 龙)", autrement dit non pas fabrication de récits, mais modelage de graphies en forme de ce qui peut figurer de façon merveilleuse (en dragons) le sens (l'esprit) de ces graphies. C'est que l'écriture y est idéographique, c'est-à-dire refaçonnage des idées par ajustement de symboles graphiques de manière à "sculpter " des images du sens des choses bien plus subtiles que celles que fabrique à l'emporte-pièce la langue parlée, outillée seulement de phonèmes. Dans cette écriture, les graphies, extrêmement sophistiquées, sont autonomes par rapport aux mots de la langue parlée; les prononciations accrochées à ces graphies ne leur sont que des accessoires de lecture. 
À partir d'une différence de nature aussi radicale, la littérature chinoise ne pouvait que se déployer dans des formes profondément étrangères à la littérature occidentale. Cependant, à l'époque moderne, les avancées décisives de la culture européenne dans les sciences et techniques ayant valu à cette culture une influence irrésistible, la littérature chinoise, en dépit de cette profonde hétérogénéité, a été pénétrée de courants littéraires d'inspiration occidentale. C'est dans ce contexte que l'œuvre de Gao Xingjian a pris corps, d'une façon qu'étudie Xiao Yingying avec beaucoup de perspicacité dans les pages qui suivent, que je suis heureux de recommander aux lecteurs.

Le premier effet de l'influence de la littérature occidentale sur la littérature chinoise a été massif: l'abandon par celle-ci de la forme d'expression écrite propre à l'idéographie, à la langue graphique (wenyan), en faveur de l'emploi exclusif du baihua, une sorte de logographie (表词文字). Celle-ci avait été extraite sous les Tang de l'idéographie originelle, déjà sous une influence occidentale, l'influence du bouddhisme indien, pour donner naissance à une littérature de langue parlée. Sans être alphabétique, cette écriture logographique, en replaçant la création littéraire entièrement dans le domaine de la parole, l'a beaucoup rapprochée de la littérature occidentale. Au lieu de "sculpter des dragons " sémantiques comme les auteurs des textes du wenxuan文词, les créateurs de la littérature en baihua, comme ceux de la littérature occidentale, se sont plu à fabriquer des récits. Tels furent d'abord les bianwen 变文, puis les xiaoshuo 小说. Et de même que dans la littérature occidentale la tragédie grecque sort de l'épopée homérique formulée théâtralement, de même des récits en baihua est sorti le zaju 杂剧 de l'époque des Yuan, où se décèle également l'influence indienne.

Rien d'étonnant à ce que, dans ce contexte, le roman et le théâtre aient été pareillement cultivés par Gao Xingjian. Il les a cultivés dans la forme nouvelle que leur avait donnée Lu Xun en les chargeant d'une dimension occidentale de subjectivité, ignorée aussi bien du baihua prémoderne que du wenyan. Cependant, dans cette dimension, Gao Xingjian fait un pas de plus que ses contemporains chinois, sous l'influence plus particulière de la littérature française contemporaine, qu'il connait bien pour en avoir été le traducteur. Ce qui a 
révolutionné celle-ci dans la seconde moitié du xx siècle, c'est justement la crise de la prédominance en Occident de la subjectivité dans l'expression littéraire, aussi forte qu'implicite depuis toujours. Une crise qui a conduit au nouveau roman et au théâtre de l'absurde. Chez Nathalie Sarraute et Robbe-Grillet, le nouveau roman n'est plus formé par le récit d'événements que traversent des personnages dont la subjectivité que crée l'auteur fait les sujets de l'histoire; il est maintenant formé de la forme même de la narration, dont l'auteur travaille essentiellement sa manière de saisir les choses. Quant au théâtre de l'absurde, de Ionesco, Becket, Genet, en déstructurant la psychologie des personnages qu'il met en scène, il réduit la subjectivité qui sous-tend les drames humains à n'être qu'une mécanique chaotique.

Cette remise en cause de l'expression traditionnelle romanesque et théâtrale de la subjectivité fait écho, littérairement, à la remise en cause philosophique de la conception cartésienne du sujet pensant. On y retrouve le point de vue de Merleau-Ponty quant à la primauté de la perception sur la conscience: ce n'est pas le moi qui pense mais quelque chose qui pense dans le moi.

Xiao Yingying montre comment tout l'œuvre de Gao Xingjian, romans et drames mais aussi encres sur papier, reflète ce courant de la culture française contemporaine. Ce qui fait peut-être de lui un écrivain trop français, même en chinois, pour être en Chine aussi apprécié que l'Occident l'a montré en le couronnant du prix Nobel.

Léon Vandermeersch 



\section{Introduction}

The more substantial an individual's aesthetic experience is, the sounder his taste, the sharper his moral focus, the freer - though not necessarily the happier - he is.

Joseph Brodsky, Discours à Stockholm

Tu n'es pas un dragon, tu n'es pas un insecte, tu n'es ni l'un ni l'autre, ce non-être, c'est toi, ce non-être n'est pas une négation, mieux vaut dire que c'est une réalisation, une empreinte, une dépense, un résultat, avant un épuisement total, c'est-à-dire la mort: tu n'es rien autre qu'un message de la vie, une expression, une parole dite envers le non-être. Gao Xingjian, Le livre d'un homme seul

\section{LA QUESTION DE LA SUBJECTIVITÉ}

Comment peut-on interroger la subjectivité en chinois alors qu'elle est si " incertaine »? Cette incertitude ne provient pas seulement de la subjectivité elle-même, mais aussi de ce sur quoi elle s’appuie. Si la subjectivité s’implique d'emblée dans le concept de "sujet " et dans la pensée de l'Être qui fondent la philosophie occidentale, qu'en est-il de la zhutixing (主体性) - traduction chinoise de la subjectivité - à quoi se réfère-t-elle dans la pensée chinoise qui échappe au dualisme sujet/objet et à la pensée ontologique? Si ce sont des événements historiques et culturels, comme la Réforme, les Lumières et la Révolution française, qui forgèrent la subjectivité en tant qu'essence de la notion d'individu, quel rôle auront joué, par comparaison, les mouvements de modernisation qui ont marqué le 
$\mathrm{xx}^{\mathrm{e}}$ siècle en Chine? Comment, sous quel régime spécifique la subjectivité pouvait-elle s'installer dans une pensée chinoise traditionnelle où la notion même d'individu n'a pas sa place?

Certes, dans la pensée chinoise, il y a aussi des notions variées du moi: chez Confucius, chez Laozi et Zhuangzi et dans le bouddhisme chán $n^{1}$, mais ces auteurs n'ont pas adopté de parti pris subjectif ou objectif, ni conçu le moi comme forme ou comme matière. Le moi confucéen est entrevu à travers les relations sociales ou privées, tandis que les formes du moi chez Laozi et Zhuangzi et dans le chán sont plus proches - elles traduisent la quête d'une disponibilité (chez Laozi et Zhuangzi) ou d'une vacuité (dans le chán) par détachement du soi-même afin d'atteindre un état de non-moi ou un état de moi impersonnel.

Le moi dans la pensée chinoise est ainsi fluide et constant à la fois. Fluide, parce qu'il n'y a pas un " quelque chose » de stable qui puisse constituer le propre du moi - c'est une perspective essentielle de la pensée chinoise de voir la nature des choses (y compris le moi) comme l'eau qui coule ou la vapeur qui flotte. Comme le dit Léon Vandermeersch: «la pensée chinoise saisit la nature des choses non pas comme sub-stancielle, c'est-à-dire, fondamentalement stable, mais comme sub-mutationnelle, c'est-à-dire comme fondamentalement changeante ${ }^{2}$.»

Le moi est constant également - non parce qu'il se transforme perpétuellement, mais parce que la façon de l'éprouver est constante: grâce à ce moi a-ontologique, la perception du moi qui est à la fois en moi et impersonnelle, sans être entravée par la distinction de chaque individu, se transfère comme l'" essence " de l'esthétique chinoise de siècle à siècle et de génération en génération. Plus précisément, la façon de penser le moi qui ne se focalise pas sur le moi et la façon de percevoir le moi qui n'assure pas de ce qui est en moi rendent, au contraire, la possibilité d'avoir aussi bien un moi qu'un non-moi.

1. Le chán (禅) est une école bouddhiste née en Chine à partir du v ve siècle, très influencée par la pensée taoïste. Le chán s'est transmis de Chine au Japon, sous le nom de zen (禅), hanja en Corée (禪) et au Vietnam sous le nom de thiền (禅).

2. VANDERMEERSCH, L., Les deux raisons de la pensée chinoise. Divination et idéographie, Paris, Gallimard, 2013, p. 111. 
Ici, les œuvres de Gao Xingjian - écrivain, dramaturge, peintre, cinéaste et aussi critique de littérature et d'art, lauréat du prix Nobel de littérature en 2000 - prises sous l'angle de la culture, s'offraient comme un terrain privilégié pour un parcours d'enquête. Parce que la poursuite de la subjectivité et la quête du moi sont chez lui au premier plan, mais aussi parce que sa façon de percevoir et d'observer le moi repose en même temps sur la perception esthétique traditionnelle en Chine et sur la construction d'un moi influencé par la notion occidentale de l'individu. Gao peut être considéré comme un auteur transculturel, et pas seulement parce qu'il écrit aussi en français. Chez lui, les deux influences de l'Occident et de la Chine, qui sont presque opposées, fonctionnent à la fois en tension et en harmonie à travers ses ouvrages.
\end{abstract}

La vie personnelle de Gao se divise en deux périodes: l'une en Chine (avant 1987) et l'autre en Allemagne et en France (en Allemagne en 1987, en France depuis 1988). L’année 1987 partage sa vie littéraire en deux, un avant (inauguré en 197833) et un après. La Chine, en tant que contexte de ses œuvres, diffère selon cet avant et cet après. Dans la première période, la Chine correspond à une expérience réelle, politique et éthique; dans la seconde, où elle ne subsiste que dans son souvenir et son imagination, elle renvoie plutôt à une expérience esthétique et culturelle. Sous ce rapport, la pièce de théâtre La Fuite écrite en 1989 peut être classée dans la première période, puisqu'elle concerne le mouvement du 4 juin; et La Montagne de l'âme (1982-1989) comme Une canne à pêche pour mon grand-père (1986) dans la seconde puisqu'il s'agit de textes plutôt esthétiques et travaillant le sensible; quant au Livre d'un homme seul où le contexte chinois implique une expérience à la fois politique et esthétique, en raison de la date d'écriture (1996-1998), il est à classer dans la seconde période.

Aussi ce travail se composera-t-il de deux parties: la première évoquera le contexte de la modernisation chinoise et le

3. Gao avait écrit antérieurement des pièces de théâtre, mais toutes ont été brûlées par lui-même pendant la révolution culturelle (1966-1976). 
développement, en lien complexe avec elle, de la subjectivité-moi chez Gao: ce dernier, à la fois, s'engage dans la modernisation et s'en retire; la partie suivante s'efforcera de cerner la notion de subjectivité-moi dans le cadre de son esthétique: il s'agira de comprendre comment Gao crée un style fondé sur le mode de percevoir chinois, tout en absorbant la pensée spéculative occidentale. La question du moi ne se focalisera plus sur la question " qui suis-je? » mais sur la question « où suis-je?".

\section{La modernité et la modernisation: l'individu et le collectif}

Les premières œuvres (nouvelles et pièces de théâtre) que Gao publie, au début des années 1980, s'inscrivent dans la deuxième vague d'occidentalisation/modernisation que connaît alors la Chine. À travers ces œuvres, il apparaît que la poursuite de la subjectivité-moi (comme pour les autres intellectuels et écrivains de cette époque) revient avant tout à la question de savoir comment envisager les dilemmes Chine/Occident et tradition/modernité, qui obsèdent depuis longtemps l'intelligentsia chinoise: l'enjeu est de sortir d'un certain enfermement sur elle-même de la pensée chinoise. Chez Gao, pionnier de l'« école moderne » du début des années 1980, la recherche de la subjectivité-moi s'incarne d'emblée dans cette question de la modernité.

Wang Hui, chercheur en pensée et littérature chinoises contemporaines, affirme dans son livre La Montée de la pensée chinoise moderne qu'il y a deux conceptions de la modernité chinoise ${ }^{4}$. L'une, en tentant de renverser le dualisme Orient (Chine)/Occident, construit son propre discours sur la modernité chinoise ou asiatique indépendamment de la modernité occidentale. Wang Hui se réfère à des auteurs tels que Liang Suming ou John King Fairbank, ainsi qu'à l'école de Kyoto (à Naito Konan notamment), en quête d'une modernité pourvue d'une motivation intrinsèque dans le cadre extrême-oriental, et de manière privilégiée autour de la pensée chinoise. L'autre conception s'efforce, à l'instar de Max Weber, dans

4. WANG, H., 《中国现代思想的兴起 》Zhongguo xiandai sixiang de xingqi (La montée de la pensée chinoise moderne), Beijing, Sanlian, 2004, p. 2-23. 
Confucianisme et Taö̈sme, de penser la modernité chinoise dans un cadre rationnel - plus précisément, celui du rationalisme issu de la tradition du capitalisme européen - propre à servir d'outil de mesure, d'observation et d'estimation des civilisations chinoise et indienne. Selon Weber, il y aurait eu déjà, en Chine, une sorte de "rationalisme politique " à l'époque des royaumes combattants ( $\mathrm{v}^{\mathrm{e}}$ siècle av. J.-C.). Manquant du support d'un rationalisme économique, ce rationalisme politique aurait trouvé sa fin dans la forme politique de l'empire, Qin et Han. Et Weber d'en conclure: la pensée chinoise ne pouvait accueillir un esprit capitaliste tel qu'il a émergé dans le cadre éthique et moral de la religion réformée européenne ${ }^{5}$.

De ces deux conceptions - l'auteur de La montée de la pensée chinoise moderne penche d'évidence vers la première -, l'une accentue la particularité chinoise d'une " modernité » enracinée dans le néoconfucianisme; l'autre, partagée par la majorité des sinologues à la fin du XIX ${ }^{\mathrm{e}}$ siècle et au début du $\mathrm{XX}^{\mathrm{e}}$ siècle, s'adosse à l'universalisme de la raison. Il y a opposition entre ces deux interprétations de la modernisation chinoise, qui s'inscrivent d'emblée dans le conflit entre un nationalisme issu de l'ethnocentrisme et un eurocentrisme issu du logocentrisme.

Ces deux tendances opposées se rencontrent encore chez des chercheurs contemporains, en Chine comme à l'étranger. Elles ne traduisent pas seulement une difficulté théorique à définir la modernité chinoise; leur conflit, dès les prémices de la modernisation chinoise, se joue sur un fond d'anxiété: comment préserver ou reformuler une identité culturelle propre, dans le cadre de cette tension, d'une part, de la morale et l'éthique traditionnelles avec la démocratie, et, d'autre part, avec la science occidentale.

Les nombreuses études de la modernité occidentale la montrent ambiguë, transitoire, fugitive, contingente ${ }^{6}$. La modernité chinoise présente les caractéristiques inverses. Avec le but bien clair de sortir

5. Ibid., p. 2-9; voir aussi WeBER, M., Confucianisme et Taoïsme, traduit de l'allemand par Jean-Pierre Grossein et Catherine Colliot-Thélène, Paris, Gallimard, 2000.

6. Voir "Qu'est-ce que les Lumières » dans Dits et écrits // de Michel Foucault, et Five Faces of Modernity: Modernism, Avant-garde, Decadence, Kitsch, Postmodernism de Matei Calinescu; également, All that is Solid Melts into Air - The Experience of Modernity de Marshall Berman. 
de l'impasse et de développer l'économie, la science, la politique et la culture, la modernité chinoise, notamment après 1949, a visé le destin du pays et de la nation, mais négligé toute recherche de l'indépendance de la personnalité. Elle apparaît de ce fait formelle, idéologique et, selon le mot de Wolfgang Kubin, «totalitaire ${ }^{7}$ ». Il est certain, en tout cas, que la modernité chinoise, tout au long du $\mathrm{xx}^{\mathrm{e}}$ siècle, a mis l'accent sur la politique et la morale plutôt que sur l'individu.

Lorsque les intellectuels chinois transposent et introduisent la notion d'individu, au moment où la Chine rencontre l'Occident à la fin du XIX ${ }^{\mathrm{e}}$ siècle et au début du $\mathrm{xx}^{\mathrm{e}}$, ils la conçoivent comme le marqueur privilégié de la modernité et la situent à l'opposé de la notion traditionnelle du moi. Le paradoxe est que, manquant de la source philosophique et de la filiation historique et culturelle d'Occident, les intellectuels chinois se retournent, à la fois inévitablement et volontairement, vers l'éthique traditionnelle et reprennent sous son égide la responsabilité de la nation et du pays - en phase avec la situation sociopolitique de la Chine d'alors. Ainsi, la volonté de faire place à la subjectivité individuelle est incorporée aussitôt à la quête de l'identité de la nation. C'est pourquoi la notion d'individu paraît souvent d'un emploi ambigu chez les écrivains de l'époque du 4 mai 1919 comme chez ceux des années 1980, notamment lorsqu'il s'agit d'envisager la liberté individuelle.

Ainsi, lorsque les intellectuels des années 1980, héritiers du mouvement du 4 mai, s'efforcent d'imaginer et de produire une littérature moderne pour sortir de la projection de la révolution culturelle, ils se retrouvent dans une situation similaire à celle de leurs devanciers, s'agissant du rapport pays, nation/individu - le premier terme va toujours à l'encontre du second. Ce dilemme du "moderne ", avec écartèlement entre la Chine et l'Occident s'incarne, chez une majorité des écrivains chinois du début des années 1980, dans le conflit idéologique entre le "réalisme " et "l'école moderne"(现代派): cela révèle justement l'« illégalité "

7. KUBIN, W., Die Chinesische literatur im 20, Jahrhundert, traduit de l'allemand en chinois: 《二十世纪中国文学史》Ershi shiji zhongguo wenxueshi, Shanghai, Huadong shifan daxue, 2008, p. 253. 
de s'exprimer en tant qu'individu dans la littérature d'alors. Cette ombre politique et morale omniprésente se projette sur les ouvrages de Gao à cette époque, bien que ce dernier souligne maintes fois sa place marginale dans la société et sa position non engagée dans la politique. Cette tendance " apolitique " de Gao lui colle d'ailleurs, paradoxalement, une étiquette politique. Ce n'est pas le propre de Gao: c'est un phénomène culturel et politique assez remarquable dans la littérature chinoise du $\mathrm{xx}^{\mathrm{e}}$ siècle.

À travers la longue période qui s'étend du 4 mai aux années 1980, sur l'exemple des ouvrages de Lu Xun à Gao Xingjian, on perçoit l'évolution du modernisme chinois. Ces deux auteurs cristallisent et promeuvent deux sortes de modernité: l'un, anticonfucianiste, n’abandonne jamais les impératifs " confucéens " de prendre responsabilité pour tout le monde (天下), et de se battre pour un futur "plus lumineux " de la Chine; l'autre a recours à une autre source de la pensée chinoise, le taoïsme et le bouddhisme, et vise la modernité dans un cadre esthétique plus qu'éthique.

Si le modernisme est un train roulant à toute vitesse, ce que nous voulons observer, ce n'est pas simplement quand et comment Gao monte dans ce train, mais comment il cherche sa propre place une fois monté dans le train, et comment, de quel point de vue il regarde défiler le paysage, chinois et occidental, et, en même temps, de quelle façon ce paysage se projette dans ses yeux.

\section{De qui suis-je? à où suis-je?: le sujet percevant a-ontologique}

Dans la plupart des romans et des pièces de théâtre que Gao a écrits dans sa deuxième période, les noms propres des personnages sont remplacés par les pronoms personnels " je ", " tu » et " il/elle ». Ces pronoms personnels révèlent et réalisent une autoprojection du moi. Par exemple, dans La Montagne de l'âme, les trois voix narratives principales tissent une relation où " tu » est l'ombre de " je ", et " elle » est l'ombre de « tu ». Ce déploiement désunifiant du moi dans une triple perspective libère l'approche d'une subjectivité qui conjoint le en-moi et le hors-moi, et favorise des expressions inédites du soi. Ce jeu à l'assise pronominale est, sur le double plan linguistique et psychologique, universel: «ce qui me paraît intéressant, 
c'est que ce triple niveau cognitif ne fait défaut à aucune langue de l'humanité. Cela montre que les trois pronoms transcendent les différences ethniques et linguistiques pour refléter la structure profonde de la conscience humaine ${ }^{8}$.»

Cette " narratologie des prénoms personnels " (nous la développerons dans deuxième partie) pose le problème de la perception dans l'expérience esthétique: comment perçoit-on « je », « tu » et « il/ elle " comme un seul et un même sujet-moi? Qui sera le vrai sujet percevant dans le processus de la perception? S'agit-il d'un « je » en moi ou bien d'un « je » hors du moi?

Rappelons ce que disait Merleau-Ponty: « De sorte que, si je voulais traduire exactement l'expérience perceptive, je devrais dire qu'on perçoit en moi et non pas que je perçois ${ }^{9}$. " Il semblerait que les pronoms personnels de Gao soient proches de cet on anonyme qui ne se réfère chez Merleau-Ponty ni au moi ni à l'autre, mais à un autre moi. Il y a, en tout cas, une similitude entre les deux approches sous le signe de cet autre moi: cet autre qui me fait éprouver l'expérience de la sensation mais ne me fait pas éprouver moi-même comme auteur de cette perception.

La différence est que là où Merleau-Ponty cherche une " nouvelle ontologie » dans son exploration de la perception, Gao construit un monde sensible qui repose largement sur la manière de percevoir selon l'esthétique traditionnelle chinoise. Aussi, si Gao croise Merleau-Ponty lorsque la question ontologique " qui suis-je? » est changée en la question contraire à toute substantialisation « où suisje? ", ils n'en divergent pas moins aussitôt: Merleau-Ponty change le monde de la raison et de la logique en un monde de la perception (le corps, le regard, l'intuition) propre à faire l'objet de la philosophie, alors que la perception, chez Gao, reste toujours un en-dehors de ce qui constitue l'objet pensé et le sujet pensant.

En effet, la question " qui est le vrai sujet percevant " n'appelle pas chez Gao la réponse d'un qui substance. Cette question motive plutôt un détachement radical de toute perspective ontologique; il

8. GAO, X. J., De la création, Paris, Seuil, 2013, p. 43.

9. Merleau-Ponty, M., Phénoménologie de la perception, dans Maurice Merleau-Ponty, Euvres, Paris, Gallimard (Quarto), 2010, p. 905. 
s'agit de faire advenir une subjectivité fluide qui s'incarne dans un sujet impersonnel percevant - ou bien dans un non-moi/sans moi percevant. Si dans les langues européennes "qui » est omniprésent dans la grammaire et la logique de la pensée, dans la langue chinoise, ce " qui » peut être absent et remplaçable sans qu'on aille à l'encontre de la structure du langage ou de la pensée: le je dans la langue et la pensée chinoises n'éprouve pas la nécessité d'être un qui. Lorsque Gao appelle son style de narration " courant de langage " en référence évidente au " courant de conscience ", cela ne veut pas dire que chez Gao « je " soit où " je parle ». Ce " je » ne s'identifie pas au je-sujet linguistique de Benveniste: "C'est dans et par le langage que l'homme se constitue comme sujet ${ }^{10}$. " Pour Gao, « je parle » n'implique pas qu'il y ait un certain « je " qui se construise à partir du langage, ni un certain lieu dans la pensée ou dans la linguistique où " je " se trouve - « je parle " pointe vers un « je » fluide qui se trouve dans plusieurs lieux.

Les expérimentations littéraires et artistiques de Gao inaugurent un regard nouveau sur la façon traditionnelle de penser et de percevoir. Si nous disons que la question " où " est une question a-ontologique, c'est parce que cet " où » exige une pensée qui ne cherche pas à se focaliser sur l'« objet " pensé. François Jullien remarque que dans la pensée chinoise, il y a un "entre " qui échappe à la philosophie occidentale, et qui constitue en revanche le goût fondamental dans l'esthétique chinoise ${ }^{11}$. Cet « entre ", est aussi une question d'، où »: il n'a rien en propre ni ne possède de statut, mais s'ouvre un passage où le moi ou les moi peuvent se déployer.

10. BenVENISTE, É., Problèmes de linguistique générale I, Paris, Gallimard, 1966, p. 259. 

PREMIÈRE PARTIE

\section{LA MODERNITÉ AMBIGUË: COMMENT LA SUBJECTIVITÉ S'IMPLANTE DANS LA LITTÉRATURE CHINOISE MODERNE}





\section{Y a-t-il du modernisme en Chine au début des années 1980 ?}

Le phénomène effrayant avec les écrivains chinois d'aujourd'hui, c'est qu'ils adoptent continuellement de nouveaux termes dont ils ne fournissent pas de définitions. Écrire longuement sur soi-même est expressionniste. Écrire sur les autres est réaliste. Écrire des poèmes sur les jambes des jeunes filles est romantique. Interdire les poèmes sur les jambes des jeunes filles est du classicisme. Tandis que "Une tête tombe du haut du ciel ", "Un bouf se tient debout sur une tête ", "L'amour! " ou " Des éclairs verts courent sur la mer! "sont du futurisme.

Lu Xun, $L^{\prime} E x$-voto ${ }^{1}$

\section{L'ÉCOLE MODERNE}

\section{Entre « école moderne " et réalisme: ambiguïtés}

En 1981, Gao publie une brochure intitulée Premier essai sur la technique du roman moderne, en souhaitant y expliquer la méthode employée dans la rédaction de ses nouvelles, alors peu comprises et confrontées à des difficultés de publication. Dix-sept chapitres composent cette petite brochure, dont le premier concerne «l'évolution du roman ». Or ce titre ne correspond pas au fond: commençant par une introduction sommaire et en même temps très vague sur l'histoire de l'évolution du roman, ce premier chapitre semble expliquer pourquoi l'auteur consacre ce livre à la « technique du

1. LU, X., "L'ex-voto» dans Fuvres complètes de Lu Xun VI, Beijing, Renmin wenxue chubanshe, 1996, p. 18; nous traduisons. 
roman » sous l'angle de l'art de la narration: « le formalisme n'est pas bon, mais il faut quand même estimer la forme ${ }^{2}$." Puis, Gao parle dans les chapitres suivants de l'art de la narration (notamment à travers l'emploi des pronoms personnels " je ", " tu " et " il/elle ») de la construction de l'intrigue, des notions de temps et d'espace, et de la perception de la vérité... Bref, l'art du roman se réfère aux courants du modernisme occidental tels que le courant de conscience, le symbolisme et l'absurde.

Ce Premier essai sur la technique du roman moderne attira immédiatement l'attention et suscita un débat autour de l'« école moderne ". Wang Meng, l'un des plus prestigieux écrivains de l'époque postrévolutionnaire, futur ministre de la culture (19861989), écrivit aussitôt une lettre à Gao et la fit publier plus tard dans Le monde du roman ${ }^{3}$. Montrant ainsi qu'il considérait Gao Xingjian lui-même comme un écrivain de l'école moderne, il admettait en même temps ne pas savoir vraiment quel était l'objet de cette école. Pour en démontrer la légitimité, Wang fournit un argument bien vague: le réalisme - la seule forme littéraire " autorisée " à cette époque -, est venu aussi d’Occident. La même année (1982), trois écrivains, Feng Jicai, Li Tuo et Liu Xinwu publient dans Littérature de Shanghai leurs correspondances, intitulées respectivement « La littérature chinoise a besoin de l'« école moderne » - lettre de Feng Jicai à Li Tuo ", "Le " roman moderne " ne s’identifie pas à l'« école moderne " - lettre de Li Tuo à Liu Xinwu " et « Nous avons besoin de réfléchir calmement - lettre de Liu Xinwu à Feng Jicai ${ }^{4}$. " Parmi eux, Feng est le plus enthousiaste: il loue chaleureusement le livre de Gao, donnant sans hésiter son adhésion à l'« école moderne » :

J'ai entendu dire que [la brochure de Gao] était un bestseller. Jusqu'alors, peu d'écrivains se sont avancés dans le jardin du « roman

2. GAO, X. J., Premier essai sur la technique du roman moderne, Guangzhou, Huacheng chubanshe, 1981, p. 2.

3. WANG, M., 《王蒙给高行健的信 » (《Lettre de Wangmeng à Gao Xingjian »), dans « 小 说界》Xiaoshuo jie (Le monde du roman), 1982, n² 2, p. 232 et p. 252.

4. Voir FENG, J. C., 《中国文学需要现代派冯骥才给李陀的信》, LI T., 《现代小说'不 等于现代派' - 李陀给刘心武的信 》, LIU X.W., 《需要冷静地思考-刘心武给冯䩀才的 信», dans 《上海文学》 Shanghai Wenxue (La littérature de Shanghaı), 1982, n 8, p. 88-98. 
moderne ", on dirait que [la brochure] surgit comme un joli cerf-volant qui se lance dans le ciel désert, quelle chose surprenante ${ }^{5}$ !

À force d'impressionner les cercles littéraires et les lecteurs, le Premier essai sur la technique du roman moderne attira l'attention de la très officielle Association des écrivains de Chine (中国作家协会). Son vice-président, Feng Mu, critiqua la brochure, dénonçant un livre " absurde ", " réactionnaire " et ennemi de la littérature réaliste socialiste. Ensuite, le journal officiel Wenhui Bao (文汇报) organisa une réunion, dite « conférence concernant la question du modernisme et réalisme » (现代主义与现实主义问题 讨论会) où furent convoqués ${ }^{6}$ Gao Xingjian, Wang Meng, Feng Jicai, Li Tuo, Liu Xinwu et une dizaine d'autres écrivains, afin de critiquer leur attitude « incorrecte " par rapport à l' " école moderne ». Puis, le Wenhui Bao publia la lettre anonyme d'un lecteur, critiquant derechef le Premier essai et l'école moderne qui menaçaient le futur de la littérature chinoise...

Le piquant, dans ce débat autour de l' "école moderne ", c’est que les deux partis opposés n'auraient su ni l'un ni l'autre définir ce qu'elle était réellement. Ni les conservateurs qui tentaient de sauvegarder le réalisme, ni les écrivains qui se prétendaient rénovateurs et applaudissaient l'avènement de la " nouvelle » forme, n'avaient une connaissance plus claire que cela de l' "école moderne " ou du modernisme. Wang Meng en fit l'aveu dans son autobiographie publiée en 2007:

Je veux reconnaître par honnêteté, qu’à cette époque, parmi ceux qui croyaient être offensés ou, au contraire, ceux qui préconisaient l'« école moderne ", et ceux qui critiquèrent sévèrement l'« école moderne ", personne n’en connaissait véritablement le sens. On ne la connaissait pas, mais on tenait à faire du bruit, en parler, la critiquer, lutter contre, se positionner et se battre maintes fois contre les mou-

5. Ibid., p. 88.

6. GAO, X. J., 《隔日黄花》, dans Ne pas avoir de -isme, «没有主义», Taibei, Lianjing ( 联经), 2001, p. 177-186; WANG, M., «王蒙自传» (L'Autobiographie de Wang Meng), livre II, chapitre 17, Guangzhou, HuaCheng chubanshe, 2007, p. 160-168; LIU X. C. (刘锡 诚), 《1982: 现代派的风波》(《1982: la désaccord autour d'école moderne »), dans 《南方文坛》 Nanfang Wentang (Le Monde littéraire du Sud), 2014, $n^{\circ} 1$; l'article entier est publié sur internet : http://www.dqlib.com.cn:8007/Template/classic/Journal_ActicleList. aspx? JournalISSN=1003-7772 \& JournalNianhao $=2014 \&$ Journalqihao $=1$ 
lins à vent... Bref, on se lançait dans un combat furieux et épuisant, sans savoir pourquoi, c'est franchement un paysage particulier que notre monde littéraire ${ }^{7}$.

En 2008, dans l'interview parue dans Métropole du sud (Nanfang Dushibao, 南方都市报), Gao a expliqué l'origine du titre de son essai:

Je n'ai même pas osé parler de «l'art du roman moderne », le terme " art » était trop sensible à cette époque, il serait impliqué dans beaucoup d'autres choses. J'ai fait exprès de prendre le titre de « Premier essai sur la technique du roman moderne », en le projetant dans le domaine technique du roman afin d'avoir le plus de sécurité possible ${ }^{8}$.

La prudence de Gao était bien raisonnée, mais sa stratégie ne fonctionna pas comme prévu: le renouvellement esthétique proposé provoqua quand même un contre-coup idéologique: l'essai de Gao atteignit sa cible même s'il s'était abstenu de tirer ouvertement ${ }^{9}$.

Dans son article « Ge Ri Huang Hua ${ }^{10}$ (隔日黄花) », qui retrace le conflit entre réalisme et école moderne, Gao impute ce conflit à la fossilisation de l'idéologie et à l'intervention politique dans le domaine littéraire. Certes, c'est là un phénomène notable de la littérature chinoise du $\mathrm{xx}^{\mathrm{e}}$ siècle. Mais au regard de l'histoire des années 1980, il semble que la politique ne suffise pas à expliquer pourquoi le réalisme occupe une telle place - souveraine - en Chine et pourquoi l'ambiguïté régnait dans l'école moderne, tant à l'égard du modernisme lui-même que du réalisme également.

L'“ école moderne " n’est jamais clairement définie par Gao; même la signification du "roman moderne " reste assez confuse dans le Premier essai sur la technique du roman moderne. Juste, au premier chapitre, l'auteur en énumère quelques caractéristiques:

7. WANG, M. L'Autobiographie de Wang Meng, op. cit., livre II, p. 167 ; nous traduisons.

8. GAO, X. J., 《对诺贝尔文学奖获得者的专访：高行健 》 (“L'Entretien avec le lauréat du prix Nobel en littérature : Gao Xiangjian ») ; voir l'article intégral sur internet : http:// houhongbinvip.blog.163.com/blog/static/13165675620091024430815/

9. Premier essai sur la technique du roman moderne devint l'une des cibles du mouvement de «lutte contre la pollution spirituelle » (清除精神污染), qui s'enclencha au début des années 1980 et prit fin en 1983.

10. GAO, X. J., 《隔日黄花》, 《没有主义» Ne pas avoir de -isme, op. cit., p. 177-186. 
Le roman ne nécessite pas de raconter une histoire;

Le roman n'a pas forcément besoin d'intrigue;

Le roman n'appelle pas à modeler le caractère du personnage;

Il n'est pas non plus nécessaire de faire un récit du personnage et de

son environnement, on trouvera dans le roman d'autres manières d'écrire ${ }^{11}$.

Derrière ces assertions tempérées, formulées sur le mode de la négation, certains modèles du modernisme occidental sont pointés: Jean-Paul Sartre a montré dans son premier roman La Nausée, que l'intrigue n'est pas nécessaire ${ }^{12}$; Franz Kafka a prouvé par son écriture que la caractérisation du personnage est accessoire; les écrivains du courant de conscience ont renversé dès le début du $\mathrm{xx}^{\mathrm{e}}$ siècle le récit traditionnel en faisant de la pensée du sujet, et non plus du monde " objectif », la vraie réalité.

Cependant, Gao n’oppose jamais frontalement le réalisme au modernisme, comme le fit Brecht luttant contre Lukacs dans les années 1930, ou comme le font, au contraire, les réalistes conservateurs qui voient dans le modernisme un monstre envahissant. Il illustre ainsi l'ambiguïté qui, chez les écrivains de l'école moderne chinoise, en tient pour une sorte de complémentarité entre modernisme et réalisme, et amène ces écrivains à se distinguer des modernistes occidentaux.

Le réalisme - ce vieil adversaire du modernisme - ne s'est jamais contenté, notamment sous l'influence de la pensée marxiste, d'occuper seulement le domaine esthétique: il est l'allié naturel de la politique, avec son principe d'identification de l'esthétique à la conception du monde. Fredric Jameson a montré que c'est la contradiction interne du réalisme lui-même qui provoqua le fameux débat entre Brecht et Lukacs au sujet du réalisme et du modernisme ${ }^{13}$. Car le réalisme prétend trouver l'expression d'une expérience

\footnotetext{
11. GAO, X. J., Premier essai sur la technique du roman moderne, op. cit., p. 4.

12. Sans doute, Joris-Karl Huysmans avait déjà prouvé dans son roman À rebours, en 1884, que l'intrigue n'est pas un facteur nécessaire; mais Sartre influença beaucoup les écrivains et les intellectuels chinois après la révolution culturelle, tandis que Huysmans est resté méconnu en Chine jusqu'à présent.

13. JAMESON, F., «Reflection in Conclusion», dans Aesthetics and Politics, London, Verso, 1980, p. 212.
} 
esthétique qui se donne comme fonction de dévoiler la vérité - cet art représentatif croit qu'il s’identifie à la vérité tout en oubliant qu'il est essentiellement une fiction. Que de fois la phrase célèbre de Stendhal a-t-elle trouvé un écho chez les réalistes: « un roman, c'est un miroir que l'on promène le long d'un chemin »! Mais cette métaphore qui identifie le roman au reflet du monde finit cependant par substituer « l'objectivité » à la fiction, donc au roman lui-même. À la fin du XIX ${ }^{\mathrm{e}}$ siècle, avec le marxisme et sa doctrine du matérialisme historique (les forces productives déterminent une certaine situation des rapports de production, et aussi des formes de conscience sociale déterminées, y compris dans l'art et la littérature), le réalisme trouve de quoi se parfaire en "réalisme socialiste ".

En Chine, « décrire la réalité » (写实) est depuis longtemps de tradition dans les romans, sans avoir jamais été érigé en un concept tel que celui de réalisme. De même, le concept de fiction n’apparaît pas avant la rencontre avec la littérature occidentale, à la fin du XIX ${ }^{\mathrm{e}}$ siècle. En d'autres termes, la notion de « fiction " en Chine ne peut pas s'identifier à celle des romans occidentaux: non seulement parce qu'il s'agit d’un mélange de légendes (传奇), de contes (传说) et de mythes populaires (神怪故事) — une " fiction collective " mais aussi parce que cette " fiction " ne s'oppose pas au souhait de " décrire la réalité ". Jacques Pimpaneau va jusqu’à soutenir que le concept de littérature n'existe pas en Chine, car les écrivains chinois n’ont cessé de faire de la littérature comme M. Jourdain faisait de la prose: sans le savoir ${ }^{14}$.

De fait, dans le premier chapitre de L'Histoire du roman chinois, Lu Xun (鲁迅) confirme d'emblée que les premiers récits « romanesques " chinois ont découlé des notes que les annalistes de la cour prenaient en recueillant bruits et murmures dans la rue ${ }^{15}$ (街谈巷语). Andrew H. Plaks met également en évidence, en citant Lu Xun et Li Zhi (李贽), un rapport assez étroit entre romans

14. PimpaneaU, J., Chine, histoire de la littérature, Arles, Philippe Picquier, 1989, p. 84.

15. LU, X., «中国小说史略》Zhongguo xiaoshuo shilue (L'Histoire du roman chinois), recueilli dans 《鲁迅全集 » Luxun quanji (Euvres Complètes de Lu Xun), Vol. 9, Beijing, Renminwenxue chubanshe, 1973. 
traditionnels et grands mémoires historiques ${ }^{16}$ (par exemple Shiji “史记 »). Si fasciné que soit Gao par la littérature occidentale, cette tradition est profondément enracinée chez lui et l'aidera à former plus tard son propre style, notamment dans La Montagne de l'âme.

Il y a, dans les romans chinois, une sorte de fiction ouverte et une façon de « décrire la réalité » également ouverte: les romanciers traditionnels n'ont sans doute pas l'intention de distinguer les récits qui sont fictifs de ceux qui " représentent la vérité » dans leur roman. Rappelons l'apophtegme du Rêve dans le pavillon rouge: quand on tient le faux pour vrai, le vrai à son tour est faux ${ }^{17}$. Ainsi Cao Xueqin, l'auteur de ce roman, écrit:

Des propos insensés comblant des fascicules

Des pleurs amers, souvent, plein le creux de la main

On dit que l'auteur est ridicule

Mais qui saura goûter la saveur ${ }^{18}$ [de ces propos]?

C'est peut-être la raison pour laquelle le réalisme ne se heurte pas en Chine autant qu'en Occident à une interrogation sur la fiction et la description de la " vérité ». D’autant plus que l'esthétique n’y est pas formée en tant que catégorie et n’y est pas « indépendante » de la sociopolitique et de l'éthique comme en Occident. C'est pourquoi aussi le réalisme a pu rapidement prendre "le pouvoir du discours " dans le mouvement moderniste en Chine, comme le sinologue américain David Der-wei Wang (王德威) le rappelle, et a été accepté sans beaucoup de dissidences par les intellectuels et les écrivains au début du $x x^{e}$ siècle, grâce à son prestige de style des Lumières face à l'obscurantisme ${ }^{19}$. Qui plus est, avec le marxisme importé d'URSS, la littérature du réalisme a pris une place particulière en faisant étroitement corps avec le système politique. Après la fondation de la République populaire, le réalisme devint la norme littéraire et

16. PLAKS, A. H., «Towards a Critical Theory of Chinese Narrative », dans Chinese Narrative: critical and Theoretical Essays (editor PLAKS, A. H.), New Jersey, Princeton University, 1977, p. 309-352.

17. CAO, X. Q., Le Rêve dans le pavillon rouge, I, Paris, Gallimard, p. 116.

18. Ibid., p. 13. Nous modifions la traduction des deux derniers vers.

19. WANG, D. W., 《写实主义小说的虚构: 茅盾, 老舍, 沈从文》(Xieshizhuyi xiaoshuo de xugou: Maodun, Laoshe, Shencongwen), Shanghai, Fudan Chubanshe, 2001. 
quasiment la seule façon officiellement autorisée de s'exprimer en littérature; il en fut ainsi pendant toute l'époque maoïste et durant les premières années du postmaoïsme.

Pour ces deux raisons, l'influence traditionnelle et le contexte politique, la littérature "réaliste " a promu une représentation littéraire qui efface la limite entre la fiction, la réalité et la vérité, et identifie l'idée de l'auteur à la parole du protagoniste. L'alliance nouée avec la politique et la morale prédispose la littérature à les servir, par sa manière d'unir étroitement la forme et le contenu - plus précisément, la forme se soumet au contenu. Cette littérature atteint son sommet pendant la révolution culturelle: par exemple dans le roman Jinguang dadao ("金光大道 ", La Voie lumineuse) de Hao Ran, dont les héros sont des figures sublimes et parfaites, et leurs adversaires des êtres effroyables et abominables, et où la voix omnisciente du narrateur représente la voix de la justice.

Mais le réalisme socialiste, de même que le réalisme révolutionnaire, devient finalement le témoin fossilisé d'une réalité limitée à ce qu'en " autorise " l'orientation de la politique. Arrivé à ce point, le réalisme qui prétendait représenter la réalité/vérité n’offre paradoxalement plus qu'une littérature irréaliste et figée. Du coup, l'école moderne du début des années 1980, qui met en avant l'expérience personnelle afin de montrer la "vraie sensation ", peut-elle apparaître plus réelle et s'impose comme le reflet de l'époque.

Les questions n'en subsistaient pas moins: quel est le caractère propre de l'école moderne? Que signifie le roman moderne: un concept historique $^{20}$ (roman de nos jours) ou un concept de révolte (roman contre le mariage de la politique et de la littérature), ou bien une catégorie possédant certains caractères particuliers?

\section{La recherche de la forme}

Chez Gao, l'expérience du "modernisme " a naturellement impacté la forme. Étudiant le français, s'intéressant spontanément

20. Dans son Premier essai sur la technique du roman moderne, bien que Gao introduise l'expression «roman moderne » dans le chapitre de «l'évolution du roman », il ne lui attribue aucune dimension temporelle précise; aucune catégorie de temps, aucun cadre géographique ne sont mentionnés, même si «moderne » renvoie en soi à un cadre temporel et fait appel en ce sens. 
à l'art littéraire, Gao s'interroge d'emblée sur la forme romanesque. S'étant doté d'une connaissance solide des mouvements littéraires occidentaux contemporains, il put réaliser que les professeurs d'université n'avaient introduit dans leurs programmes que les romans occidentaux classiques des XVIII et $\mathrm{XIX}^{\mathrm{e}}$ siècles: personne ne parlait des romans contemporains. Grâce à sa connaissance de la langue française, Gao lut pour ainsi dire tout le corpus de la littérature occidentale à la bibliothèque de l'université, y compris des documents de consultation réservée. Il écrit dès alors beaucoup ${ }^{21}$ mais ses amis écrivains le découragent de publier ses manuscrits qui tendent au formalisme et seraient inévitablement considérés comme dangereux et politiquement incorrects. Gao mit ainsi un pied dans le " roman moderne " dès les années 1950, mais il lui fallut attendre 1978, la période post-maoïste pour réintroduire subtilement l'école moderne: les anciennes générations d'écrivains d'alors n’osaient plus parler de "modernisme " après ces années de bouleversement, et les jeunes n'en avaient même pas connaissance.

En 1979, Gao accompagne l'écrivain Ba Jin (巴金), en tant qu'interprète, lors d'un voyage officiel en France. À Paris, il voulut rendre visite aux " grands maîtres » du modernisme:

Je voulais voir Duras, on m'a dit qu'elle était très vieille; et j'ai dit que Jean Genet m’intéressait beaucoup, on m’a répondu qu'il était agonisant à l'hôpital. Je me suis rendu compte tout à coup de la gravité de l'écart temporel entre la Chine et l'Occident. Les modernistes occidentaux étaient déjà comme Lu Xun de notre pays, décrits dans les manuels scolaires comme événement historique ${ }^{22}$.

Cette visite parisienne fut un grand choc pour Gao; elle lui donna matière à s'exprimer, dans les débats ultérieurs, sur la réalité de l'« école moderne » chinoise eu égard au modernisme occidental:

De nos jours, pourquoi discutons-nous encore de la légitimité de l'« école moderne »? L'" école moderne » et le modernisme ne sont plus en Occident quelque chose de moderne. Ces gens-là [les modernistes

21. Gao avait écrit une dizaine de pièces de théâtre, un scénario et plusieurs nouvelles qu'il a brûlés pendant la révolution culturelle.

22. GAO, X. J., LI, O. F., LI, T. et CHENG, A., «作家四人谈》 ( La discussion entre quatre écrivains »), dans 《文学自由谈» Wenxue Ziyoutan, TianJin, 1986, n5, p. 25-36; nous traduisons. 
occidentaux] devraient communiquer avec Ba Jin, et non pas avec nous $^{23}$.

Gao s’aperçut très vite de ce " décalage horaire " entre Chine et Occident: au début des années 1980, alors que le modernisme est considéré comme quelque chose de dépassé, et que le postmodernisme ou le poststructuralisme prévalent en Occident, la Chine est en train de flotter et de polémiquer autour du réalisme et du modernisme.

Mais si Gao place Lu Xun et Ba Jin au même niveau que Duras et Genet, en y voyant les représentants des " modernistes » de génération antérieure, les deux groupes d'écrivains n’en sont pas pour autant si comparables, pour le style et pour le fond. Le « décalage horaire " ne peut résumer toute la différence existante entre l'école moderne chinoise des années 1980 et le modernisme occidental.

En réalité, le modernisme s’était déjà fait jour en Chine dans les années 1920 avec une inflexion particulière. Ce modernisme chinois se différencie contextuellement du modernisme occidental, issu de Baudelaire, Proust, Kafka et T. S. Eliot, qui prit son essor à la fin du $\mathrm{XIX}^{\mathrm{e}}$ et au début du Xx ${ }^{\mathrm{e}}$; il fait suite au mouvement de la " nouvelle culture ». Les premiers écrivains modernistes chinois, qui constituent l'école de la « nouvelle perception », tels Mu Shiying (1912-1940), l'auteur de Shanghai de hubuwu (Le foxtrot à Shanghai), Shi Zhecun (1905-2003), l'auteur de Jiangjun de Tou (La tête du générale) et Liu Na'ou (1905-1940), l'auteur de Dushi fengjingxian (Paysage urbain) durant les années 1920 et 1930, introduisent et adaptent le symbolisme, l'expressionnisme et la psychanalyse (transités par le Japon). Ils sont très rapidement considérés comme les représentants scripturaires de la bourgeoisie capitaliste, à l'opposé du réalisme considéré comme la littérature de gauche (左翼文学). Globalement, le modernisme ne s'est donc pas déployé amplement dans la première moitié du $x^{\mathrm{e}}$ siècle. C'est le roman réaliste, comme on l'a dit précédemment, qui s'impose comme le courant littéraire dominant en Chine, particulièrement à partir de 1949, la naissance de la République populaire marquant le commencement de la littérature chinoise contemporaine.

À la fin des années 1970 et au début des années 1980, en cherchant à relégitimer le "modernisme ", les écrivains de l'école

23. Ibid., p. 27. 
moderne sont obligés, d'une part, de chercher un compromis avec le réalisme-socialiste auquel ils s'opposaient, et, d'autre part, de trouver le moyen de dialoguer avec la culture contemporaine occidentale: ainsi, ils doivent à la fois se soumettre à l'impératif que le récit reflète l'idéologie, selon le « leitmotiv de l'époque (时代主旋 律) ", et le concilier avec la nouvelle forme narratologique. D’où, en particulier, la contradiction relevée entre forme et contenu dans les premières créations littéraires de Gao.

Sa première longue nouvelle, Étoile dans la nuit froide ( 寒夜的 星辰 »), écrite en 1978, relate l'expérience d'un vieux communiste pendant la révolution culturelle. Prisonnier de ses camarades au nom de la Révolution, le héros souffre de la mort de sa femme, de celle de ses amis et de sa séparation d'avec ses enfants; dévasté par la «stigmatisation » et la violence de l'« autocritique et de la critique publique ", il meurt finalement maltraité à l'hôpital.

L'Étoile dans la nuit froide est écrite à la première personne du singulier: "je ", l'ami du héros, est le seul interlocuteur de ce vieux communiste, et il se fait l'interprète des fragments du journal intime du héros. Gao fera appel à l'un des pères du socialisme, Engels, pour légitimer son style et sa divergence par rapport à celui du réalisme socialiste:

Engels n'agrée pas que le personnage soit le porte-voix de l'esprit de l'époque [...] Je crois qu'Engels avait raison. La littérature peut représenter l'esprit de l'époque, mais ne doit pas nécessairement le propager directement à travers le personnage, ce qui finirait par en simplifier les richesses et réduirait sa vie à un seul thème... La perspective de l'Étoile dans la nuit froide est celle du narrateur « je " qui se fixe sur les derniers dix ans de ce vieux communiste. Dans ce cas-là, le narrateur « je » ne raconte pas ce qu'il ne savait pas, et ne joue pas le rôle d'un narrateur omniscient ${ }^{24}$.

Dans cette nouvelle, en modifiant la perspective du narrateur, Gao essaye de dénouer le lien entre le personnage principal et le narrateur: ce dernier n'est plus le "microphone » de l'« esprit du temps ». Néanmoins, malgré cette volonté, Gao ne parvient pas totalement à faire disparaître le narrateur omniscient. L’ami du « vieux

24. GAO, X. J., dans la postface d'Un pigeon s'appelle bec rouge, Beijing, Shiyue wenyi chubanshe, 1984, p. 336-337. 
communiste " qui fait office de narrateur est un personnage qui de ce point de vue en sait trop: il est plus qu'averti des souvenirs, des sensations personnelles et même du processus psychologique de son cher ami.

Gao a perçu sans doute cette contradiction entre la nouvelle forme qu'il cherchait et un contenu politiquement contraint. Le vieux communiste d'Étoile est le seul héros chez Gao qui attache sa vie étroitement à la politique, et qui soumet sa vie privée à son identité sociale. Conséquence, le compromis nécessaire entre l'écriture privée (le journal et le souvenir personnel) et le " grand idéal » du héros qui voulait rendre au peuple la responsabilité de chercher le bonheur pour le plus grand nombre, détermine une incohérence entre le récit fragment, psychanalytique, et le récit tendant à une " grande narration ".

Un pigeon s'appelle bec rouge (《有只鸽子叫红唇儿»), l'autre nouvelle écrite en octobre 1980, juste avant le Premier essai sur la technique du roman moderne, et après sa visite à Paris, raconte les histoires d'amours brisées, de vies bouleversées et d'esprits torturés de six jeunes amis pendant que se déroule le cataclisme politique et économique des années 1950-1970. Kuaikuai, un jeune génie, est forcé de se séparer de son amoureuse Yanping, à cause de son père « droitiste " (右派); Yanping est obligée de se marier avec un jeune homme issu du même milieu communiste, qu'elle quittera finalement pour tenter de rejoindre Kuaikuai qui mourra de surmenage au travail après la révolution culturelle. Un autre protagoniste surnommé Coq, croyant qu'il vit dans une grande époque, s'engage à plein dans le mouvement. Il perd son amie Xiaoling qui ne supporte pas la rupture avec sa famille exigée par le jeune homme, et se noie dans une rivière. Zhengfan, le personnage sans doute le plus lucide, abandonne les études supérieures et devient ouvrier pour nourrir sa mère et sa jeune sœur. Cette dernière, par folie et naïveté, se jette dans la révolution et devient indifférente à la violence, mais finalement souffre de malaise vis-à-vis des gens affectés par ce mouvement.

Le rôle du narrateur est ici plus varié que celui du narrateur de l'Étoile: tantôt il raconte l'histoire aux lecteurs, tantôt il discute avec les six protagonistes en tant que personnage, tantôt il dialogue avec chacun d'eux, vivant ou mort, à travers le temps et l'espace et selon son bon vouloir. Aussi cette nouvelle est-elle composée de 
discours, directs ou indirects, et de séries de correspondances entre les personnages racontant leur propre histoire: la voix réunie des six personnages permet finalement au narrateur de fabriquer l'histoire " comme multi-parties de composition musicale ${ }^{25}$ ». Un pigeon s'appelle bec rouge est ainsi l'essai d'une " polyphonie " qui sera plus tard la forme principale des pièces de théâtre de Gao, telles que L'Homme sauvage, La Fuite et Quatre quatuors pour un week-end. Le désaccord entre la forme et la thématique est très adouci dans cette nouvelle. Il n’y a plus de héros parfait, mais des jeunes anodins, excités, peureux, flegmatiques ou simplement perplexes devant leur époque.

Entre un récit à tendance subjective et un événement objectif, le médiateur est indispensable. Dans l'Étoile, le narrateur-personnage accepte de tendre au rôle de narrateur omniscient, qui s'impose finalement contre la volonté de l'auteur. Également, dans Un pigeon, le changement de la position du narrateur ne peut aboutir. Gao se permet d'intervenir comme un personnage au début de l'histoire. Rappelons que le premier chapitre commence par la voix de l'auteur:

La parole de l'auteur

Ce n'est pas une nouvelle écrite de la manière traditionnelle, bien qu'elle raconte toujours l'histoire du destin humain.

Il y aura six personnages dans la nouvelle. En été 1957, Kuaikuai, Coq et Zhengfan ont bientôt fini leurs études au lycée. Kuaikuai, le plus jeune parmi eux, avait seulement 16 ans. Il y a aussi trois autres filles: Yanping, Xiaoling et la petite $\operatorname{sœur}^{26}[\ldots]$

Il semble que cet auteur-narrateur est inspiré par le rôle d'“ annonceur-narrateur (说书人) " du xiqu (l'opéra traditionnel, 戏曲) et du roman chinois traditionnel. Andrew H. Plaks remarque que cet "annonceur-narrateur " provient toujours du "narrateur » des mémoires d'histoire (par exemple, Taishigong 太史公 de Shiji) qui apparaît à la fois comme l'annonceur objectif déployant l'histoire et comme le critique/analyste de l'histoire ${ }^{27}$. Mais cet " annonceur-narrateur" des romans et des opéras (xiqu 戏曲)

25. GAO, X. J., Un pigeon s'appelle bec rouge, Beijing, Shiyue wenyi chubanshe, 1984, p. 337 26. GAO, X. J., Un pigeon s'appelle bec rouge, op. cit., p. 1.

27. Plaks, A. H., 《中国叙事学》(La Narratologie chinoise), Beijing, Beijing Daxue Chubanshe, 1995, p. 16. Ce recueil a servi de base aux séminaires de Plaks à l'université de Beijing en 1989. 
s'attribue plus de liberté: il entre et sort librement du récit/intrigue, soit qu'il prédise l'histoire aux spectateurs, soit qu'il critique les personnages, soit qu'il explique le monde psychologique du personnage. Dans Un pigeon s'appelle bec rouge, cet annonceur-narrateur établit même une " convention " entre personnage et lecteur:

Selon le principe du roman traditionnel, il faut un héros. Nous pourrions élire Kuaikuai, ce génie qui meurt précocement [...] Ce livre cite principalement les propres paroles de six protagonistes. S’y ajoute la parole du narrateur qui dialogue parfois avec les protagonistes; les lecteurs qui s'ennuieraient de ces compléments pourront s'en affranchir. Il convient que l'auteur respecte les différents goûts des lecteurs ${ }^{28}$.

Cette combinaison des rôles sera reprise et développée dans les pièces de théâtre comme Chronique du Classique des montagnes et des mers et les romans La Montagne de l'âme et Le livre d'un homme seul - ces romans proposeront une narratologie particulière usant de la " trinité » des pronoms personnels « je », « tu » et « il/elle », qui révèle le doute de Gao, s’interrogeant sur le rapport entre subjectivité et perspective: la subjectivité correspond-elle à une perspective?

\section{La littérature des cicatrices ${ }^{29}$ ?}

Les effets produits par la quête d'un nouveau style dans ces deux nouvelles ont été ignorés par les critiques de l'époque ${ }^{30}$, qui rattachèrent ces textes à la " littérature des cicatrices ${ }^{31}$ ". Li Shifei, le directeur de la maison d'édition de Huacheng, en se préparant à publier les deux nouvelles, pensa d'ailleurs qu'elles relevaient trop de ladite littérature des cicatrices et proposa à Gao d'ajouter quelques autres nouvelles pour atténuer la sensation doloriste.

28. GAO, X. J., Un pigeon s'appelle bec rouge, op. cit., p. 2.

29. La littérature des cicatrices (伤痕文学) est apparue en Chine à la fin des années 1970, donnant une vision crue et directe de la société chinoise, marquée notamment par le sinistre de la révolution culturelle.

30. À l'exemple de la critique des deux nouvelles par l'écrivain Feng Yidai (冯亦代) : une critique qui porte seulement sur l'histoire, non pas sur la forme que Gao expérimentait et qui était assez particulière dans la littérature chinoise d'alors. Voir “这也是历史” ("C'est aussi l'histoire »), dans Dushu ( «读书»), Beijing, 1983, nº 7 .

31. Su Chen : 《高行健在花城起步 » (《Gao Xingjian s'est enclenché à Huacheng »), dans 《粤海风》Yue naifeng, 2008, nº 6, p. 53-55. 
Il faut relativiser cette catégorisation. Certes, ces deux nouvelles relatent l'une et l'autre les souffrances de la révolution culturelle, mais l'intention d'Un pigeon s'appelle bec rouge n'est pas simplement d'évoquer la mémoire de l'époque: il s'agit encore de chercher, à travers les intrigues fragmentées et les perceptions des personnages, la subjectivité de l'auteur et celle de la "nouvelle littérature ». D’autant plus qu'au niveau de la langue et du style, Un pigeon ne porte pas les marqueurs de la mise en accusation de la Bande des Quatre, et se distancie du langage post maoïste.

La littérature des cicatrices, surgissant au terme de la révolution culturelle, exorcise le sinistre en exprimant une blessure sentimentale et le mécontentement face à la réalité. Ce mouvement littéraire éphémère répondait au besoin de défoulement collectif en exposant la plainte "du sang et des larmes " formulée par les victimes de l'époque, mais en même temps il prévenait la mentalité officielle: la catharsis opérait en visant la Bande des Quatre considérée comme responsable de la révolution culturelle; la littérature des cicatrices aidait l'autorité à désigner une cible concrète, et ce faisant se rapprochait de la représentation réaliste asservissant l'esthétique au jugement de valeur. Les premières œuvres de la littérature des cicatrices apparurent à la fin des années 1970; elles employaient souvent une sorte de langage révolutionnaire lorsqu'elles accusaient la révolution culturelle: phrases remplies par des points d'interrogation menant directement à la " conclusion » rituelle et simpliste que la Bande des Quatre empoisonnait la nation. Parmi les plus connues, on peut citer L'Enseignant de la classe ("班主任 》) de Liu Xinwu, ou Cicatrices ( “伤痕 ") de Lu Xinhua, dont l'image de martyr ou de victime servait d'axe narratif. Autour de cette dichotomie catastrophe/ martyr, ces auteurs accusaient la violence patente de la révolution via une autre violence, latente dans le texte: leur langage, calque paradoxal du langage révolutionnaire maoïste, porte une vision hiérarchique qui sublime la valeur de la nation et de l'État: l'intérêt de ces derniers est toujours supérieur à celui de l'individu. La littérature des cicatrices se conforme donc à un modèle identique: en montrant les souffrances, en prenant le point de vue optimiste romantique, elle se consacre à nouveau au destin de la nation et du 
pays et n'en doute point; elle l'impose encore et toujours comme un futur lumineux et commun à tous les Chinois.

Si Étoile dans la nuit froide est encore quelque peu en phase avec la littérature des cicatrices, Un pigeon s'appelle bec rouge n'y adhère plus: à la fin de la nouvelle, en dialoguant avec l'annonceur-narrateur, un des protagonistes, Coq, avoue que la littérature sert peu de guide à la vie. Ce qui signifie, en fait, que l'auteur fait émerger la conscience de l'individu qui refuse d'être assimilé à une quelconque idéologie.

\section{QU'EST-CE QUI SOUTIENT LA NOUVELLE FORME?}

\section{Dans un parc}

De 1982 jusqu'à la veille de son exil en 1987, les nouvelles que Gao écrit apparaissent comme une collection d'exercices de style: les récits fragmentaires évoquant une sensation personnelle se substituent aux intrigues (Sur la mer (海上), 1983; Insulte (侮辱), 1983; La crampe (抽筋), 1984); les histoires se dévident sous forme de dialogue ou de monologue sans voix du narrateur [La pluie, la neige et l'autre (雨、雪及其他), 1982; HuaDou (花豆), 1982; Mère (母亲), 1983; Dans un parc (公园里), 1983; Une canne à pêche pour mon grand-père (给我老爷买鱼竿), 1986]. S'ajoutent les descriptions « indifférentes » d'une scène quotidienne [En route (路上), 1982; Vingt-cinq ans après (二十五年后), 1982; Couronne de fleurs (花环), 1983; Le cordonnier et sa fille (鞋匠和他女儿), 1982; Le Temple de Yuan En (圆恩寺), 1983; L'Accident (车祸), 1983].

Ces nouvelles n'éclairent pas de façon trop insistante l'arrière-plan historique et interviennent peu dans les problèmes sociopolitiques; elles tentent surtout de décrire des fragments d'émotions en expérimentant les différentes formes possibles de narration dans tout l'éventail de rôles du narrateur. Par exemple, Dans un parc commence par un dialogue:

- Il y a longtemps déjà que je n’ai plus flâné dans un parc. Je n’ai jamais de temps libre et ça ne me dit rien non plus.

- Tout le monde en est là, le travail fini, on fonce chez soi, on est toujours pressé.

- Je me souviens, quand j'étais gosse, j'adorais venir dans ce parc faire des roulades sur l'herbe. 
— Amené par tes parents ${ }^{32}$.

La voix du narrateur n’est plus le vecteur nécessaire à la promotion de l'intrigue; elle est presque absente; l'histoire se déploie à travers le dialogue de deux protagonistes et exige aussi la participation du lecteur: la disparition de la voix du narrateur oblige le lecteur à remettre en question toutes les confidences. Puis un récit s'insère soudainement dans le courant de la conversation:

Sous les arbres qui bordent les deux côtés de la grande allée est plantée une haie de cyprès bien taillés; sur la pente, de l'autre côté des cyprès, une jeune fille vêtue d'une robe et portant un sac à main rouge s'assied sur un banc de pierre ${ }^{33}$.

Le dialogue coule selon son propre rythme, mais la scène à la fille s'insère maintes fois:

— Le soleil va bientôt se coucher.

- Oui, c'est beau.

- Je n'aime pas ce genre de beauté dans un cadre artificiel.

- Mais tu disais que tu adorais venir au parc.

- C'était quand j'étais petit. Depuis, j'ai vécu en montagne, j'ai été bûcheron dans la forêt vierge, pendant sept ans.

[...]

La fille en robe se lève de son banc, scrute l'extrémité de l'allée, pardelà les cyprès impeccablement taillés. Quelques personnes s'en viennent de ce côté-ci; parmi elles, un jeune homme de haute taille dont les cheveux longs couvrent les tempes [...]

- Ça fait longtemps que je n'ai pas vu un coucher de soleil aussi beau, on dirait que le ciel prend feu.

— On dirait un incendie ${ }^{34}[\ldots]$

L’intrigue ne se développe pas seulement au gré du dialogue des deux partenaires, elle est aussi dynamisée par l'action de la fille en robe rouge qui opère un contraste: les deux se parlent, la fille garde le silence; les deux restent assis, la fille est active; les deux n’apparaissent pas concrètement, la fille est décrite en détail dans son apparence. Ce type de contraste - le récit parallèle qui est très

32. GAO, X. J., 《公园里》 (《Dans un parc ») ; la version chinoise a paru dans «南方文学 》 Nanfangwenxue, 1985, n² 4, regroupée avec cinq autres nouvelles de Gao dans Une canne à pêche pour mon grand-père, L' Aube, 1996, traduit du chinois par Noël Dutrait, p. 43.

33. Ibid., p. 44.

34. Ibid., p. 44-45. 
développé dans les pièces de théâtre et les romans de Gao -, offre plusieurs visions en décomposant la perspective du narrateur, afin d'observer plus directement l'état psychologique et sentimental des personnages. Ainsi, ce à quoi les deux partenaires font face n'est plus un monde extérieur comme dans les romans réalistes, mais un monde intérieur représenté par la fille en robe rouge qui " dialogue » sans parole avec les deux dialogueurs. Dans ce " dialogue ", les deux côtés (les deux partenaires du dialogue et la fille en robe) sont comme deux sujets intériorisés: en parallèle au début, et se croisant à la fin (la fille en robe attire finalement l'attention des deux partenaires). Le lecteur est lui aussi invité à intervenir dans l'histoire en la construisant. Le monde objectif - le réseau de relations, le statut social - perd de son importance. Aussi n'y a-t-il pas de nom propre dans la nouvelle: l'emploi libre des pronoms personnels convient mieux à un tel changement rapide de la perspective. Les « je ", " tu », « il/elle ", à la place des noms de personnages, désignent les intervenants, mais impliquent aussi bien tout un chacun, y compris le lecteur lui-même. Il en va ainsi dans les quatorze nouvelles ci-dessus mentionnées, dont douze sont animées par des protagonistes anonymes.

Pour autant que le nom propre renvoie d'abord à une identité sociale discernable, sa disparition révèle le désir de se retirer de cette relation et de se tourner vers le monde intérieur. Il y a une tendance à marginaliser et à estomper progressivement l'identité sociale chez Gao: dans Étoile, le héros, le vieux communiste, est ministre; dans Un pigeon, les protagonistes sont les six jeunes amis, étudiants ou ouvriers; dans ses deux premières pièces de théâtre, Signal d'alarme et L'Arrêt d'autobus, les héros sont un jeune chômeur (Heizi) et des voyageurs sans nom. De même, dans les quatorze nouvelles écrites plus tard (La Crampe, La Pluie, la Neige et l'Autre, Dans un parc et Une canne à pêche pour mon grand-père), l'identité des protagonistes devient encore plus équivoque et plus floue. Passant du supérieur hiérarchique aux personnages anonymes et marginalisés, Gao s'éloigne de plus en plus du réalisme socialiste. Lorsque le récit de la vie individuelle se détache ainsi de la chaîne sociopolitique, il gagne en dimension esthétique et conquiert une nouvelle liberté. 
Mais, pour autant, cette tendance à la personnalisation esthétique n'est en rien le révélateur d'une indifférence de l'individu envers la société, telle celle qu'éprouve le héros de L'Étranger de Camus, ni d'un antagonisme envers elle comme celui d'Érostrate chez Sartre. Dans un parc ne prône pas la rébellion contre la morale et l'éthique, et ne cherche pas non plus à atteindre le style de l'esthétique pure. Dans sa recherche et expérimentation d'un nouveau style, Gao n'oublie pas la douleur produite par les mouvements sociaux, exprimée par la bouche d'un personnage: "si je me suis mariée avec lui, c'est parce que je ne pouvais pas trouver mieux. Ce sont ses parents qui se sont débrouillés pour me faire revenir en ville ${ }^{35}$ ». Dans Le Cordonnier et sa fille, l'auteur accuse un père ignare et têtu qui interdit à sa jeune fille de prendre contact avec aucun jeune homme, causant ainsi une tragédie mortelle; quant à En route, c'est une satire des bureaucrates; et Vingt-cinq ans après montre une scène mélancolique où le protagoniste rencontre son ancienne amoureuse après tous les bouleversements de l'époque. Gao reste donc dans l'ambiguïté du "nouveau »: en tendant à la nouvelle esthétique dite moderniste, il garde ce qui dérive de la tradition réaliste - l'humanité et la compassion.

Par comparaison avec ses nouvelles, le théâtre de Gao a d'ailleurs davantage tendance à intervenir dans les problèmes sociaux. Ses deux premières pièces, L'Arrêt d'autobus et Signal d'alarme, écrites en 1981-1982, sont considérées comme les premières pièces d'avant-garde jouées en Chine; elles transmettent bien "l'ambiance " de l'époque. L'Arrêt d'autobus raconte, entre réalisme et absurde, comment un groupe de passagers désireux de se rendre à la ville attend en vain depuis dix ans dans un arrêt d'autobus abandonné; Signal d'alarme décrit la bataille psychologique qui se livre dans le cœur d'un jeune chômeur, HeiZi, complice d'un brigand des voies ferrées, lorsqu'il rencontre par hasard, dans le wagon de marchandises qu'il a l'intention de piller, son vieil ami venant d' " hériter » du poste d'employé du chemin du fer, accompagné de celle qu'il aime - la famille de la fille refuse leur relation à cause de son chômage.

35. Ibid., p. 50. 


\section{L'innovation de la forme et la réforme de la société}

Zhang Xudong (张旭东) remarque, dans sa monographie Chinese Modernism in the Era of Reforms, que l'apprentissage de la culture occidentale dans les années 1980 en Chine consiste en un exercice d'imagination collective chez les intellectuels concernant le «nouveau monde " - un monde imaginaire représentant la culture occidentale avancée. Zhang estime aussi que le "style " était la façon la plus commode et la plus facile de participer au «nouveau monde ${ }^{36}$ ». Certes, par l'innovation du style et de la forme, les intellectuels chinois d'alors se "connectent " directement à leurs homologues occidentaux. Mais en même temps, à la fin des années 1970 et au début des années 1980, l'école moderne imagine avoir à viser un nouveau futur à partir de la réalité chinoise au lieu de construire un "nouveau monde " en suivant l'Occident; dans la poursuite de la nouvelle forme, les modernistes chinois n'ont pas renoncé à leur intérêt pour la réalité.

D'ailleurs, Gao lui-même estime que l'innovation formelle n'était pas sans importance eu égard précisément à la réalité: « À l'époque, en Chine, tout débat sur la forme a revêtu, pour la société, un sens aigu $^{37}$. " En effet, l'une des raisons importantes pour laquelle on appelle l'époque des années 1980 la "nouvelle ère ", c'est justement la réforme du style qui provoqua la réflexion sur la littérature et même sur la politique - la question de la démocratie. Gao l'a confirmé: " [...] pour dire les choses autrement, comme il n'était pas facile de "dire vrai", pour éviter de toucher directement à des tabous, il fallait faire un détour, en parler par le biais de la forme ${ }^{38}$. "

Concluant sur les traits culturels des années 1980, Gan Yang, chercheur en pensée et littérature chinoises contemporaines, montre que

36. ZHANG, X. G., Chinese Modernism in the Era of Reforms, Duke University Press, 1997, p. 334: "'Style'proves to be the most convenient and the cheapest way of experiencing and participating in the new world. More often than not, it is through the cultural products of the contemporary West that the social grammar of the modern is captured by the social imagination ». Zhang est professeur en littérature comparée à l'université de New York.

37. GAO, X. J., et YANG, L., Visite à Gao Xingjian et Yang Lian, traduit du chinois par Chantal Chen-Andro, d'après la conversation de Gao avec Yang en automne 1993 à Sydney, Paris, Caractère, 2004, p. 21.

38. Ibid., p. 19. 
la réforme économique n'était pas encore devenue un objet de discussion parmi les intellectuels; leurs débats se concentraient presque tous dans le domaine des sciences humaines; la philosophie et la littérature occidentales ${ }^{39}$ étaient l'objet de toutes les attentions. Mais pas de façon " gratuite ": les discussions des années 1980, y compris sur la forme "moderniste ", répondaient au sentiment de la nécessité d'une réforme sociale - dont l'une des conséquences les plus connues a intégré la mémoire collective planétaire: Tian’an men en 1989.

Ainsi, la volonté de réforme du style n'évoluait jamais dans le seul domaine esthétique pur, entité abstraite qui est trop loin de la réalité en Chine. Le soin apporté au style était le marqueur de la poursuite de nouvelles idées. De ce point de vue, les pronoms personnels qui remplacent les noms propres et qui n'apparaissent presque jamais au pluriel chez Gao (il y a " je », « tu », « il/elle ", mais pas « nous » et " vous »), révèlent qu'il privilégie l'individu au détriment de la volonté collective du nationalisme et du patriotisme.

Comme l'innovation formelle était une façon indirecte de contrecarrer la politique totalitaire et d'en sortir, elle ne pouvait désormais ignorer la relation à nouer avec la politique et la réalité sociale. Ce rapport entre l'école moderne et la société est à l'antipode du modernisme occidental, dont le tropisme " antisociété » est un caractère distinctif essentiel. Il est à se demander, dans le cas chinois, quel sens pourra revêtir l'innovation formelle, une fois levés les interdits.

Le modernisme, aux yeux de Gao, se signale par l'imbrication de la forme et du contenu et par le fossé d'incohérence qui finit par séparer la théorie esthétique et la réalité du monde. Après sa visite à Paris en 1979, Gao voit dans cet état de faits le symptôme de la crise du modernisme en Chine. Il écrit un article deux mois après le voyage, intitulé "La douleur de la littérature moderne française », qui s'ouvre sur les questions sartriennes: « Qu'est-ce que la littérature? À quoi sert-elle? Ce sont les questions que la littérature française se pose à elle-même après la Seconde Guerre mondiale ${ }^{40}$

39. ZHA, J. Y. (dir.), «八十年代访谈录》 (Interviews des années 1980), Beijing, Sanlian shudian (三联书店), 2006, p. 196.

40. GAO, X. J., 《法兰西现代文学的痛苦》 (《La douleur de la littérature moderne française »), dans 《外国文学研究》 Waiguo wenxue yanjiu, 1980, n 1, p. 51-57; nous traduisons d'après la version chinoise. 
[...] ». En s'appuyant sur l'idée que « l'idéologie est le reflet de l'infrastructure économique de la société dans la superstructure ${ }^{41}$ ", Gao n'était pas en symbiose avec Sartre, bien que son article ait été certainement inspiré par Qu'est-ce que la littérature?: "Sartre propose une littérature engagée [...] Il voit que l'on a besoin de la violence révolutionnaire pour changer la société, il souffre pourtant de la destruction et de la mort produite par les conflits violents. De même, il appelle la réaction, mais il se débat en même temps dans la spéculation de la morale et ne peut s'en sortir ${ }^{42}$. " Néanmoins, Gao n'était pas non plus très positif concernant l'adversaire de Sartre, Alain Robbe-Grillet. Ce romancier représentatif du nouveau roman ne faisait que se débattre dans le bourbier de la forme: "Cette poursuite extrême de la forme conduit le roman à sa destruction ${ }^{43}$."

Gao s'est servi de la littérature moderne française comme d'un miroir pour contempler la littérature chinoise: ni la littérature engagée ni la pure réforme du style ne pouvaient désormais répondre aux questions qu'il posait à la littérature moderne française, et également à la littérature chinoise hésitante au seuil du modernisme, ainsi qu'à lui-même en tant qu'écrivain "moderniste ». De plus, dans le contexte sociopolitique influencé par la pensée marxiste avec impératif de cohérence entre la culture et l'idéologie, chercher uniquement une réforme du style apparaît une impasse aux yeux de Gao. Le besoin est celui d'une narratologie " ouverte ", d'un texte " ouvert » accueillant des contenus variés, à l'opposé du texte " arbitraire " portant le sceau d'une idéologie autoritaire. Situation ambiguë: l'individualisme qui fut la motivation interne du modernisme occidental n'avait pas encore trouvé sa base théorique, sociale ou historique à cette époque en Chine. La quête de la forme, manquant de motivation interne mais poussée par la confrontation idéologique et l'intervention sociale, était condamnée à perdre paradoxalement sa force propre une fois que la tension entre modernisme et réalisme se détendrait.

41. Ibid., p. 57.

42. Ibid., p. 52

43. Ibid., p. 56. 
À cet égard, deux ou trois ans seulement après le débat autour du Premier essai sur la technique du roman moderne, les conservateurs du réalisme font un compromis avec les écoles modernes: en 1985, la pièce d'avant-garde La visite d'un mort à un vivant dont l'auteur, Liu Shugang (刘树钢), obtient le grand prix du théâtre, est considérée comme " une bonne combinaison de la tradition et du moderne ". La même année, les nouvelles de Liu Suola Tu n’as pas de choix (“你别无选择”) et de Xu Xing Variation sans thème (《无主题变奏》) sont publiées, et considérées comme l'acte de naissance de vraies œuvres modernistes en Chine.

Pour autant, il semble que les écoles modernes ne l'aient pas emporté au final. Ainsi, la littérature d'avant-garde, après avoir conquis la place dominante au théâtre et dans le roman, perd vite de son importance: au début des années 1990, le théâtre expérimental ne parvient plus à attirer l'attention du public. S'agissant des romans modernistes, la situation n'était pas meilleure. Perdant de plus en plus de lecteurs et menacés par les nouveaux impératifs de la commercialisation, les écrivains d'avant-garde cessent d'écrire, se réconcilient avec le réalisme ou s'orientent vers la démarche universitaire, notamment à la fin des années 1990.

Pour en revenir à Gao, c'est dès le milieu des années 1980 - alors que l'école moderne dont il fut l'un des initiateurs alimente une nouvelle vague de modernisation/occidentalisation et donne corps à l'un des plus remarquables courants littéraires de la Chine contemporaine — qu'il commence à prendre ses distances.

\section{QU'EST-CE QUE LA MODERNITÉ CHINOISE?}

\section{Un modernisme retardé?}

Gao Xingjian fit une communication intitulée "Le modernisme retardé et la littérature chinoise actuelle " lors du colloque "Littérature chinoise moderne et modernisme " qui eut lieu à Hong Kong en 1987, à la veille de son exil en Europe. Dans la version publiée, Gao présente le résumé qui suit de la diffusion de la littérature moderne occidentale en Chine du 4 mai 1919 aux années 1980: 
Les mouvements de littérature moderniste occidentale se sont développés de la fin du XIX ${ }^{\mathrm{e}}$ siècle jusqu'à la Deuxième Guerre mondiale. La littérature chinoise au cours de la même période, c'est-à-dire depuis ce qui a précédé et suivi le 4 mai, jusqu'aux années 1930, années de prospérité, a cherché à combiner le romantisme et le réalisme critique (批评现实主义) occidentaux des XVIII ${ }^{\mathrm{e}}$ et XIX ${ }^{\mathrm{e}}$ siècles avec la réalité sociale chinoise, jetant ainsi les bases solides de la littérature moderne chinoise. À part Lu Xun (鲁迅), Li Jinfa (李金发) et quelques autres, presque tous les écrivains chinois ont échappé à l'influence du modernisme occidental. Le discours sur le modernisme, déjà révolu en Occident, a eu lieu vers le début des années 1980, années du renouveau de la littérature chinoise. Ce modernisme occidental retardé et les courants littéraires occidentaux apparus après la Deuxième Guerre mondiale ont stimulé énormément notre littérature contemporaine en quête de renouvellement. Un courant appelé « école moderne » à la tête duquel se tiennent des écrivains de la nouvelle génération a alors déferlé dans la littérature chinoise ${ }^{44}$.

L'hypothèse du "modernisme retardé » fait donc fonds sur l'image d'une modernité du 4 mai réduite à l'écho de l'esprit des Lumières, à la manière du romantisme et du réalisme des XVIII et XIX ${ }^{\mathrm{e}}$ siècles, et d'une " école moderne " des années 1980 inspirée par le modernisme occidental proprement dit. Quelques lignes plus loin, avant de résumer les caractéristiques du modernisme chinois de son époque, Gao commence par parler de la réalité de la société:

Le modernisme dans la société industrialisée occidentale, est une révolte contre la tradition culturelle chrétienne. La Chine dont plus de $80 \%$ de la population est paysanne, dispose d'une tradition culturelle orthodoxe enracinée, basée sur le rationalisme éthique confucéen. L'évolution de la littérature vers l'époque moderne, a certainement des points communs avec celle de la littérature occidentale, mais il n'est pas possible qu'elle prenne un chemin identique à son homologue occidental.

Ce qui apparaît dans la littérature chinoise contemporaine, dite l'“ école moderne ", en général, comporte beaucoup de différences avec le modernisme occidental. Il faut analyser de manière concrète l’« école moderne » qui est issue de la réalité chinoise avec ses com-

44. GAO, X. J., 《迟到的现代主义与当今中国文学 》 (《Le modernisme retardé et la littérature chinoise actuelle »). La version chinoise a paru dans Critique de la littérature ( «文 学评论 »), 1988, n 3; elle est reprise dans 《没有主义», Taibei, 2001, p. 108-119. Nous prenons la version française traduite par Liu Fang, dans Littérature chinoise, trimestre 1, 1989, p. 58, avec certaines modifications tenant à la version chinoise de référence. 
posantes de culture traditionnelle. D’abord l'école moderne réaffirme l'existence de l'individu, au lieu de le renier, comme l'a fait le modernisme occidental. L'école moderne apprécie avec une émotion pathétique nietzschéenne la valeur de la personnalité de l'homme, au lieu de disséquer assez froidement la nature humaine. Elle s'oppose à l'éthique féodale et valorise la rationalité de l'amour sexuel, mais refuse de rejeter toute l'éthique et de repousser tout amour sexuel. Elle dénonce l'absurde de notre vie sans pourtant le considérer comme l'existence même. Elle renie la raison et affirme le subconscient, mais ne prête aucune attention, à l'irrationalité. Elle préconise le culte de la virilité sans, bien sûr, jamais suspecter la virilité comme on suspecte la nature humaine. Elle exagère son sentiment de solitude, mais ne le dirige pas vers le nihilisme. Gémissant ou pas, elle éprouve toujours une profonde souffrance. Parfois elle se moque d'elle-même et garde pourtant quelques traces de pitié et d'héroïsme crucifié. Le point de départ du modernisme occidental, c'est le doute sur l'ancien humanisme, tandis que l'" école moderne » chinoise, empreinte d'esprit romantique, tâche de retrouver dans la réalité sociale chinoise l'humanisme perdu depuis un certain temps ${ }^{45}$.

Telles que les décrit Gao, les grandes caractéristiques de l’école moderne ne diffèrent guère de celles de la littérature du 4 mai: même si les influences occidentales relèvent dans l'un et l'autre cas de strates distinctes, on retrouve en partage l'esprit romantique et l'humanisme. Pour Gao, la ressemblance des deux époques est à chercher dans un facteur principal: les conditions socio-économiques. Tout au long du $\mathrm{xx}^{\mathrm{e}}$ siècle, la Chine se présente comme une société agricole en cours d'industrialisation, ainsi que Li Zehou ${ }^{46}$ le rappelle: "les Chinois luttaient contre les privations, leur priorité était la recherche de la nourriture, et non celles du sens de la vie ${ }^{47}$ ". Rien à voir avec le contexte occidental de naissance du "modernisme ", fruit de l'urbanisation.

Il y a deux points remarquables dans ce texte de Gao. En premier lieu, le « décalage " temporel qu'il constate entre le modernisme

45. Ibid., p. 59 ; nous mettons en gras la dernière phrase.

46. LI, Z. H. (李泽厚, 1930 -) est un spécialiste chinois de la philosophie allemande (Kant) et de l'histoire intellectuelle chinoise. II est considéré comme un des intellectuels les plus importants des années 1980.

47. LI, Z. H., «中国现代思想史论 » (De l'histoire de la pensée de la Chine moderne), Beijing, Sanlian shudian, 2008, p. 278. 
chinois depuis le 4 mai et le modernisme occidental ne se réfère pas à un temps linéaire. En effet, les courants littéraires (romantisme, réalisme, symbolisme, influences de Nietzsche ou de Freud, et aussi dadaïsme et surréalisme) qui se succèdent en Occident, sont importés ou réimportés presque simultanément en Chine, au début du $\mathrm{xx}^{\mathrm{e}}$ siècle comme au début des années 1980. Comme l'explique Muriel Détrie:

En Europe, la modernité est apparue comme l'aboutissement d'une séquence suivie de mouvements littéraires: la modernité selon Baudelaire venait dépasser l'opposition du classicisme et du romantisme qui s'étaient succédé, et le modernisme du début du xx $x^{\mathrm{e}}$ siècle venait clore la série romantisme-réalisme-naturalisme-symbolisme qui avait marqué tout le siècle précédent ${ }^{48}$.

De ce point de vue, les deux époques (4 mai et années 1980) partagent une même expérience de la perception du temps quant au cours de l'occidentalisation: les séquences linéaires " passé-présent-futur » vécues en Occident sont compressés en en Chine dans la catégorie du "présent ".

Deuxième point: lorsque Gao qualifie le modernisme chinois de " modernisme retardé », en reliant étroitement la naissance de l'« école moderne " au contexte socio-économique et en situant là l'origine de la divergence des modernistes chinois avec ceux d'Occident, il montre qu'il définit la modernité — au moins la modernité de la littérature - dans le cadre global de la modernisation de la société. Ce point de vue correspond à la conception générale que partagent les intellectuels des années 1980, en accord avec deux prémisses: la culture est en cohérence avec l'économie, comme sont en cohérence l'esprit et la matière. Rappelons ce que Ye Junjian (叶君建, 1914-1999, professeur en littérature et traducteur de Hans Christian Andersen) disait dans la préface au Premier essai sur la technique du roman moderne: "Prenons les informations de la littérature mondiale actuelle avec laquelle nous communiquons et dans laquelle nous intervenons, c'est aussi un aspect non négligeable de notre modernisation ${ }^{49}$.»

48. CURIEN, A., (dir.), Écrire au présent, débats littéraires franco-chinois, Paris, Maison des sciences de l'Homme, 2004, p. 247.

49. GAo, X. J., Premier essai sur la technique du roman moderne, op, cit., p. 6. 


\section{L'individualisme au début des années 1980 interprété par Li Zehou}

Du 4 mai aux années 1980, la littérature chinoise - du moins celle qui témoigne de la construction d'un pays moderne - s'attarde peu sur la contradiction qui se creuse entre développement scientifique et existence humaine, ou entre rationalisation et subjectivation; à la différence de la modernisation occidentale, elle s'implique trop dans la poursuite de la démocratie et du développement social pour cela. Le commentaire de C. T. Hsia sur les écrivains du 4 mai (ils concentrent trop leur regard sur la politique et la société chinoises et cette focalisation les empêche de voir au-delà comme le font les écrivains modernistes occidentaux contemporains ${ }^{50}$ ) convient tout aussi bien pour dresser le portrait des écrivains du début des années 1980.

Li Zehou a vu dans la littérature des années 1980 la descendante de celle du 4 mai, leur point commun étant la défense de l'humanité; il voulait y retrouver, pour la littérature des année 1980, la légitimité de l'« individualisme » via l'époque du 4 mai, en déclarant renoncement à la révolution culturelle issue de "l'idéologie de la féodalité paysanne empruntant la forme de la révolution ${ }^{51}$ ».

Toujours selon Li, après la longue histoire de la société féodale et une courte période semi coloniale, le fait que la Chine se hâte d'entrer dans la société socialiste sans passer par le stade du capitalisme pose le problème de l'acceptation et l'intégration des idées occidentales dans la culture chinoise:

50. HSIA, C. T. argumente en décrivant le caractère des intellectuels du 4 mai dans $A$ History of Modern Chinese Fiction, p. 536 : «It would seem that he is equally concerned with spiritual sickness, but whereas every modern writer of England, America, France, and Germany automatically identifies the sick state of his contry with the state of man, in the modern world, the Chinese writher sees the conditions of China as peculiarly Chinese and not applicable elsewhere. He shares with the modern Western writer a vision of disgust if not despair, but since his vision does not extend beyond China, at the same time he leaves the door open for hope, for the importation of modern Western or Soviet ideas ans systems that would transform his country from its present state of decadence. If he had the courage or insight to equate the Chinese scene with the condition of modern man, he would have been in the mainstream of modern littérature. » 51. LI, Z. H., De l'histoire de la pensée de la Chine moderne, op. cit., p. 1-46; nous traduisons du chinois. 
Pour ainsi dire, la construction sociale et psychologique issue de cette longue histoire féodale, n’a pas été détruite par l'idée de la démocratie et de l'individualisme provenant de la société capitaliste. Les anciennes puissances et les idéologies restent irréductiblement dans notre couche profonde de la conscience et même de l'inconscient, et se reconstruisent sous le nom d'anti-individualisme et d'anti-libération capitaliste, en se déguisant en collectivisme socialiste ${ }^{52}$.

L'idéologie de la féodalité paysanne que Li accuse la théorie marxiste d'avoir réveillée et couverte est, à n'en point douter, la culture confucéenne. D’après Li, cette idéologie de la féodalité paysanne atteint l'acmé de son expression pendant la révolution culturelle, en tant qu'ennemie sans concession de l'individualisme et de la démocratie nourris par l'économie-culture capitaliste. La révolution de 1966-1976, marquée par l'anéantissement total de la culture traditionnelle, notamment celle du confucianisme, est ainsi considérée paradoxalement par Li comme une résurgence virulente de la vieille idéologie, dissimulée sous « l'aspect du collectivisme socialiste ". Qu'en est-il au vrai de ce vieux fantôme traditionnel qui viendrait s'immiscer dans le processus de modernisation et de rencontre avec l'Occident? Si par tradition il faut entendre une structure culturelle sédimentaire influant sur le mode d'action et le mode de pensée dans la vie quotidienne, on ne peut ni la rejeter comme un objet extérieur, ni la tenir pour quelque chose d'immobile.

La pensée de Li Zehou se faisait l'écho d'une opinion majoritaire chez les intellectuels au début des années 1980: il faut poursuivre le chemin de la modernisation via l'accueil de l'influence occidentale. À la différence du jeune Lu Xun, Li croit que l'idée de Neisheng ${ }^{53}$

52. Ibid., p. 35.

53. Neisheng et Waiwang forment une paire de notions qui se trouve au centre du confucianisme. Voici l'explication d'Anna Cheng: "La famille étant perçue comme une extension de l'individu et l'État comme une extension de la famille, et le prince étant à ses sujets ce qu'un père est à ses fils, il n'y a pas de solution de continuité entre éthique et théorie politique, la seconde n'étant qu'un élargissement de la première à la dimension communautaire. Confucius convertit ainsi l'autorité du prince en ascendant de l'homme exemplaire, de même que le " décret céleste » est converti de mandat dynastique en mission morale. En conséquence, la pensée confucéenne a toujours opéré sur le double registre de la « culture morale personnelle » qui vise à la « sainteté intérieure » (Neisheng) et la charge d'« ordonner le pays » qui tend à l'idéal institutionnel de la « royauté extérieure » (Waiwang) ». Voir CHENG, A., Histoire de la pensée chinoise, Paris, Seuil, 1997, p. 79. 
(内圣) qui demande une haute exigence morale à l'individu, a peu de chance d'être réalisé alors que l'Occident se contente d'une contrainte minimale accordant la loi à l'individu en tant que citoyen. Il s'ensuit que le moralisme confucéen qui repose sur l'humanité ne correspond plus du tout au système politique et juridique de la société moderne caractérisé par le contrat social ${ }^{54}$.

Pourtant, en rejetant l'idée de Neisheng, Li ne délaisse pas celle de Waiwang (外王) comme idéal politique:

Du 4 mai à nos jours, la littérature moderne fondée sur la langue orale, qui balaie les immondices féodaux et qui nettoie l'esprit national, hérite en même temps des éléments positifs de notre tradition, tel que le sentiment de patriotisme et de responsabilité envers le peuple et le pays... [puisque] même si nous nous trouvons déjà dans une situation de paix, faire prospérer notre pays, c'est-à-dire, la modernisation, est toujours la première mission pour les Chinois ${ }^{55}$.

En d'autres termes ce que Li propose, l'" individualisme ", est une notion qui dans la filiation chinoise n'est pas identique à l'individualisme qu'on trouve chez Locke ou chez Descartes, ni même à celui de Don Quichotte ou de Faust. Même si l'individualisme chez Li met l'accent sur la valeur de l'individu et sur le contrat social, la modernisation du pays et de la nation est toujours « la première mission ». Il semble que Li ne s'est pas posé la question de savoir, dans le cas où la modernisation de la Chine n'aboutirait pas, si la recherche de la valeur propre à l'individu échouerait elle aussi ?

Il reste encore un paradoxe chez Li, de même que chez tous les intellectuels ou presque des années 1980. La notion d'individu n'est jamais prise sous l'angle des résistances à la modernisation; la réflexion sur la conscience de l'individu ne conduit pas à la quête de sa libération en le détachant de l'état immature qu'il s'impose à luimême, ni à la conquête du droit de cet individu, mais à un nouveau compromis: l'individu s'implique dans le processus de modernisation où la figure de l'individu s'unit encore une fois à la figure de la nation-État. La modernité de l'individu — autrement dit, la subjectivité individuelle - est laissée au second plan et n'est représentée

54. LI, Z. H., De l'histoire de la pensée de la Chine moderne, op. cit., p. 44.

55. Ibid., p. 42-45. 
que par une modernité globale: la culture, la politique, le développement de la nation et du pays.

\section{Le moi submergé et le moi expansif}

Une soumission de la subjectivité de l'individu à la modernisation du pays ou, pour le dire autrement, " une combinaison étrange où cohabitent la subjectivité et la conscience collective ${ }^{56}$ » se projettent dans les ouvrages de Gao comme une ombre immense. Combien de fois Gao déclare-t-il qu'il la rejette, combien de fois explique-t-il encore pourquoi il y renonce: une telle tradition demeure omniprésente comme le fantôme qui n'a plus de corps-entité, mais reste toujours là. Nous verrons les traces de ce questionnement dans ses essais, les pièces de théâtre L'Autre rive, La Fuite et son dernier roman Le Livre d'un homme seul. Cela ne révèle pas seulement la difficulté de détacher le moi individuel du moi collectif, mais aussi la difficulté de définir l'" intellectuel chinois ". Et qu'est-ce donc que le moi lorsqu'il se dépouille du costume de l'« intellectuel chinois »?

\section{Le nous et le moi}

Six ans après la publication de La poursuite d'un théâtre moderne, dans une communication à un colloque sur le thème "Le pays, la société, l'individu " tenu à l'université de Stockholm, Gao exprime à nouveau son doute à l'égard du sujet « nous »:

Je me méfie quand on parle au nom du collectif, mais ce qui m'angoisse, c'est que je serais tué sans aucun bruit à ce nom de collectif. L'« intellectuel chinois » est également un substantif collectif, que je ne peux représenter et par lequel j'ai peur d'être représentés7.

Ce «nous " révèle ainsi l'obstacle difficile à franchir pour les intellectuels chinois depuis l'époque du 4 mai:

$\mathrm{Au}$ moment où ils confirment leurs propres valeurs, ils doivent se charger du fardeau de sauver le pays et le peuple: est-ce le destin obligé des intellectuels chinois? Pourraient-ils avoir un meilleur destin autrement? Pourraient-ils, lorsqu'ils affirment la valeur d'individu indépendant, ne pas être les sauveurs ni comme les sacrifiés ${ }^{58}$ [du pays]?

56. JIN, S. Y., «L'écriture subjective dans la littérature chinoise contemporaine », op. cit. 57. GAO, X. J., «个人的声音》 (《La voix de l'individu»), dans «没有主义» (Ne pas avoir de-isme), Taibei, Lianjing, 2001, p. 97 ; nous traduisons.

58. Ibid., p. 98. 
Selon Gao, ce qui produit cette fatalité tragique chez les intellectuels chinois n'est pas seulement dû à la politique et à la culture, mais aussi à la faiblesse des intellectuels eux-mêmes. Les effets d'une connaissance impropre de la notion d'individualisme et de ce qu'ils sont eux-mêmes se révèlent dans la mise en scène d'un « moi » expansif et enthousiaste:

Car ce que signifie "l'individu" pour les intellectuels chinois du 4 mai, ce n'est qu'une passion romantique qui n'est pas encore digérée en connaissance raisonnable: cet «individu " provient plutôt de la philosophie nietzschéenne du surhomme que du rationalisme français des Lumières; il s'agit plutôt d'un sentiment tragique de l'individu suprême que de l'individualisme. Ce moi qui vient de se réveiller est tellement fragile: une fois qu'il se confronte à la nation et au pays ou bien à l'intérêt collectif de classe, il s'y intègrerait avec peu de difficulté, puis s'enflerait comme un grand Moi, et se prétendrait le porte-parole de la nation, du pays et de la classe. Ainsi l'indépendance de l'esprit individuel s'engloutit dans la conscience de la nation et de la classe. C'est pourquoi, le radicalisme révolutionnaire de Lu Xun, le libéralisme de Hu Shi, l'enthousiasme de Guo Moruo pour le communisme et la capitulation de l'impérialisme de Zhou Zuoren, se trouvent tous finalement à l'abri du drapeau "sauver le pays et le peuple ", même si parfois c'est seulement pour se sauver soi-même. Cela est le caractère particulier des intellectuels chinois ${ }^{59} \ldots$

Notons-le, le romantisme est un thème que Gao critique autant que le nationalisme, le patriotisme et le totalitarisme: il signifie un autre pouvoir absolu né de l'autoperception imaginaire. Car le romantisme "a tenté d'ériger " le dieu moi » en place des pouvoirs religieux, politique et clanique ainsi que de l'intérêt national ${ }^{60}$ ».

Mais comment un tel moi romantique et enthousiaste peut-il être intégré si facilement par une idéologie et être submergé par le moi collectif partout en Chine, du 4 mai à la révolution culturelle, et même au début des années 1980 ? De quoi s'agit-il, qu'est-ce donc que l'individualité et la subjectivité pour les intellectuels chinois? Habermas cite Friedrich Schlegel afin de démontrer que dans l'art romantique, il y a un moi décentré « pour lequel tous les liens sont

59. Ibid., p. 99 .

60. GAO, X. J., «没有主义» (Ne pas avoir de -isme), op. cit., p. 136; voir aussi Visite à Gao Xingjian et Yanglian, op. cit., p. 31. 
rompus et qui ne peut vivre que dans la félicité que procure la jouissance de soi-même ${ }^{61}$ ». Il semble que ce n'est pas le cas du moi «romantique » dans le contexte chinois: il ne s'agit pas d'un moi qui rompt les liens avec les autres, mais d'un moi pour lequel la notion d'autre est impensable.

\section{Le moi romantique}

Gao a imaginé un dialogue entre lui-même et le fantôme de Mao Zedong dans Le Livre d'un homme seul, en faisant voir combien la pensée de Mao le fait sombrer dans la dépression:

Aujourd'hui, tu écris paisiblement, ce que tu veux dire à cet empereur qui a dominé des centaines de millions de personnes, c'est que, comme tu es minuscule, l'empereur qui est en toi ne peut plus dominer qu'une seule personne: toi-même. Aujourd'hui, prononçant publiquement ces paroles, tu es sorti de l'ombre de Mao, mais la chose ne fut pas facile ${ }^{62}$.

En effet, le concept du moi chez Mao a été très influencé par ce moi « romantique » qui apparaît particulièrement dans sa jeunesse, entremêlé à une pensée très traditionnelle quant à la question du rapport moi/univers.

Dans le commentaire que le jeune Mao a fait entre 1917-1918, de l'essai Le système de l'éthique de l'auteur allemand Friedrich Paulsen, il déclare que l'individu possède une valeur suprême. Car, « s'il n’y a pas d'individu, il n’y aura pas d'univers (宇宙), ainsi, la valeur de l'individu est plus grande que celle de l'univers ${ }^{63}$ ». À travers ce "syllogisme " qui paraît évidemment faux, on voit pourtant comment le je de Mao qui provient de la pensée traditionnelle se greffe sur la notion occidentale d'individu.

Je dans la culture chinoise n'est pas pensé dans la position vis-àvis de l'autre — déjà, l'utilisation de « je » et du « tu » apparaît assez

61. HABERMAS, J., Le Discours philosophique de la modernité, traduit de l'allemand par Rainer Rochlitz et Christian Bouchindhomme, Paris, Gallimard, 1988, p. 21.

62. GAO, X. J., Le Livre d'un homme seul (traduit du chinois par Noël et Liliane Dutrait), Paris, l'Aube, 2000, p. 510.

63. MAO, Z. D., 《伦理学原理批注》 (Commentaires sur 'Du système de l'éthique), voir 《毛泽东早期文稿》(Premiers écrits de Mao), Hunan, Hunan Renmin Chubanshe, 1989, p. 141-142 et p. 153 : les commentaires de Mao sont écrits sur les marges des pages du Système de l'éthique. 
tardivement dans la langue classique, tandis qu'" elle ", n'est pas employé avant le $\mathrm{xx}^{\mathrm{e}}$ siècle ${ }^{64}$. Chez Confucius et aussi dans la pensée du taoïsme, le je n'implique ni un sujet ni un moi psychologique, mais un état ouvert du moi. Cet état ne tient ni à un certain point de vue, ni à un parti-pris, ni à une forme stable, mais se transforme en suivant les changements de l'univers: en ce sens, un tel moi peut brouiller la limite entre soi et l'autre, et permet une extension sans frontières jusqu'à s'unir au ciel/univers (天人合一). C'est la raison pour laquelle nous disons que la relation moi-univers s'établit sur une certaine ignorance d'autrui. Il est compréhensible que lorsque ce moi rencontre le je de la philosophie occidentale fondé sur le dualisme sujet/objet, moi/autre, il se rabat facilement sur le parti-pris du je égocentrique, mais néglige en même temps la contrainte du moi.

Ainsi, Mao remplace le rapport moi-univers par « je suis l'univers » lorsqu'il découvre le moi-sujet dans la pensée de Paulsen: « Je tenais jadis à la théorie de "sans moi" où il n'y a que l'univers. Maintenant je me rends compte que j'avais tort: parce que je suis l'univers ${ }^{65}$ ". Cela peut expliquer la conclusion de Mao: « La valeur de l'individu est plus grande que la valeur de l'univers. » Quant à la valeur suprême de l'individu, selon Mao, il faut « la réalisation du moi » pour l'atteindre. Il s'agit d'une autonomie de la morale: «Je crois que l'on n'a que le devoir pour soi-même [...] J'ai le devoir de mettre en pratique ce que ma pensée atteint. C'est-à-dire, sur ce dont j'ai conscience, j'ai le devoir de le pratiquer ${ }^{66}$. " Rappelons le moi similaire qui correspond au surhomme de Nietzsche chez Lu Xun. En fait, un tel moi romantique apparaît chez beaucoup d'autres intellectuels et écrivains au début du Xx ${ }^{\mathrm{e}}$ siècle: Guo Moruo ${ }^{67}$ en est sans doute un exemple typique.

64. C'est Liu Ban'nong qui a utilisé pour la première fois « elle » dans son poème écrit en 1920 《教我如何不想她》 (Comment pourrais-je ne penser pas à elle?), dans LIU B. N., «刘半 农诗选 » (poèmes choisis de Liu Ban'nong), Beijing, Renmin wenxue chubanshe, 1958. En effet, 她 (elle) est un caractère, pris dans le Dictionnaire de Kangxi ( 《康熙字典》), signifiant « sœur », Liu l'a utilisé en tant que pronom personnel « elle » pour ne pas confondre avec « il ». 65. MAO, Z. D., «讲堂录》 (Notes de cours), cité par Li Zehou, dans De l'histoire de la pensée de la Chine moderne, op. cit., p. 131.

66. MAO, Z. D., Commentaires du système de l'éthique ( «伦理学原理批注 »), op. cit., p. 230231.

67. 郭沫若 (1892-1978), poète, écrivain, dramturege, archéologue, spécialiste de la Chine antique et aussi l'homme politique, l'auteur du poème romantique Déesse (Nüshen). 
Certes, derrière ce moi expansif, il y a des exhortations morales et éthiques traditionnelles, qui se fondent sur la supposition d'une nature humaine identifiée à la bonté Ren (仁) - le Ren, concept le plus essentiel du confucianisme, propose un travail permanent sur soimême (修身) tel que l'on soit à même d'étendre sa mansuétude à son entourage (ou aux autres) sans demander forcément la réciprocitée ${ }^{68}$. Cette bonté implique en effet, dès le début, une relation "inégale " qui s'appuie sur une morale d'autonomie ou d'autoperfection.

Il ne serait pas incompréhensible que ce moi autonome rejoigne le moi romantique: ils impliquent tous les deux l'ignorance de l'autre. De plus, l'homme visant l'autoperfection n'est pas le contraire d'un moi expansif - au contraire, ils se reflètent l'un dans l'autre et révèlent une tendance dangereuse à « la liberté absolue et une fausse individualité ». Car, d'une part, la confusion du devoir et du droit de l'individu permet au moi extensif sans limite de trouver la " logique » qui lui fait substituer sa propre volonté à celle des autres au prétexte de les « sauver »; d'autre part, par manque d'un point d'appui rationaliste, cette « individualité » admet une certaine déficience de la moralité pour rester un individu indépendant en face de la volonté collective, au même prétexte sublime de "sauver le pays, sauver le peuple».

En précisant la question du moi qui est effectivement le point-clé de la modernité, Gao se méfie de ce « moi romantique »:

Le romantisme, en prônant le «moi», l'avait opposé à Dieu, aux masses, afin d'affirmer pleinement sa valeur. Où commence la « modernité » de la littérature moderne? Précisément avec la remise en cause du «moi » romantique. Une telle attitude est le signe de la naissance de la littérature moderne. Aussi, derrière la succession de toutes sortes de formes, trouve-t-on un fil conducteur, une même question qui se détache et à laquelle sont confrontés nos contemporains: celle de la connaissance du "moi ", de la remise en cause du " moi », des recherches sur le « moi » et non celle du « moi » comme mythe, tel que le voyait le romantisme ${ }^{69}$.

C'est pourquoi Nietzsche lui apparaît comme le symbole de la passion romantique extravagante, et non de l'esprit moderne:

68. CHENG, A., Histoire de la pensée chinoise, Paris, Seuil, 1997, p. 68-71.

69. GAO, X. J., et YANG, L., Visite à Gao Xingjian et Yang Lian, op. cit., p. 29-30. 
Pour moi, le modernisme ne commence pas avec Nietzsche, mais après lui, et ce qu'on entend par "modernité » est précisément la remise en question de Nietzsche ${ }^{70}$.

Le moi romantique révèle en effet un statut immature sous lequel on ne peut se connaître proprement soi-même. C'est pourquoi les intellectuels chinois n’ont pas distingué le soi du pays. Aussi Gao conclut-il:

Les intellectuels chinois sont courageux: rien ne peut les arrêter lorsqu'ils combattent contre l'éthique féodale et le pouvoir bureaucratique. En revanche, ils ont les mains liées devant cette folie moderne du culte de la nation ${ }^{71}$.

À travers les critiques que Gao formule à l'encontre des intellectuels chinois - la passion du patriotisme ou du nationalisme, la conscience ambigüe de soi-même ainsi que du pouvoir totalitaire et de l'attitude radicale en face de la tradition - apparaît en filigrane ce qu'il cherche: c'est la subjectivité individuelle qui lui donnera la possibilité de se débarrasser d’un tel culte du « collectif ».

\section{La littérature ne peut être que la voix d'un individu}

Tout au long du $\mathrm{Xx}^{\mathrm{e}}$ siècle en Chine, les ouvrages philosophiques traduits de l'Occident les plus populaires sont sans doute ceux de Nietzsche et de Rousseau, avec leurs concepts respectifs du surhomme et de la volonté générale ${ }^{72}$. Les deux auteurs, dont les pensées s’opposent à maints égards, sont pourtant estimés en Chine, à l'époque du 4 mai et dans les années 1980, sur un pied d'égalité. Quant aux œuvres du modernisme contemporain ou du postmodernisme, elles n’ont pas été traduites jusqu’aux années 1990. La

\section{Ibid.}

71. GAO, X. J., «La voix de l'individu », dans «没有主义» (Ne pas avoir de -isme), Taibei, Lianjing, 2001, p. 101 ; nous traduisons du chinois.

72. Dans son discours inaugural en tant que premier président de la République de Chine, Sun Zhongshan (孙中山) proclamait: "Subvertir le gouvernement autocratique de Manchu, consolider la République de Chine, chercher le bonheur du peuple, c'est la volonté générale des citoyens de la Chine 》 ( 《颠覆满清专制政府, 巩固中华民国, 图谋民生 幸福, 此国民之公意》). Ici, la 《 volonté générale » est issue de la pensée de Rousseau. Quant à la pensée du « surhomme » de Nietzsche, elle fut diffusée rapidement en Chine grâce à Lu Xun. Néanmoins, leurs œuvres ne sont pas toutes traduites en chinois, et les intellectuels comprennent les deux auteurs à la lumière de la culture chinoise. 
diffusion des théories de Nietzsche et de Rousseau reflète l'identité de désir des intellectuels des années 1980 par rapport à l'époque du 4 mai: les concepts de la modernité sont semblables: il s'agit d'un désir qui mêle paradoxalement le "surhomme " de Nietzsche et la "volonté générale " de Rousseau, afin de développer la société. En d'autres termes, on cherche la conscience de l'individu sous l'angle du « surhomme ", mais on se place en même temps dans une dynamique où la modernisation de la littérature chinoise suppose la quête de la démocratie et de la science, et où le concept de subjectivité individuelle s'intègre dans celui de nation. Pour les écrivains des années 1980 comme avant pour ceux du 4 mai, ce qui est en cause n'est pas le souci de l'existence personnelle, mais le souci du pays et du destin de la nation.

Gao ne souscrit ni à Nietzsche ni à Rousseau — pour le dire plus précisément, il ne rejoint pas ce que les intellectuels chinois comprennent de Nietzsche et Rousseau. Il s'agit selon lui d'un « esprit romantique " attribuant à la littérature en elle-même le rôle dirigeant et la souveraineté spirituelle:

Si Nietzsche a déclaré la mort de Dieu, c'était le «moi» qu'il respectait; la littérature chinoise d'aujourd'hui, elle, n'a aucun besoin encore une fois de substituer le moi à Dieu [...] On se trouve à une époque où l'ancienne conception de la valeur disparaît très vite. Pour notre littérature, il vaut mieux connaître plus à fond notre moi et la littérature elle-même. Il ne faut pas surestimer la littérature qui n'est qu'une des représentations de la culture de l'humanité; les hommes de lettres ne sont ni des réprimandeurs ni des saints auréolés. Une fois expulsée de l'esprit de nos hommes de lettres la conscience inadéquate d'être des héros-créateurs du monde, nous atteindrons une littérature véritable, ni emphatique, ni maniérée ${ }^{73}$.

Cette volonté de détrôner la littérature aussi bien que le " moi ", à partir de laquelle Gao développe sa pensée, le conduit à la défiance envers la modernisation en Chine. Mais, en même temps, la compréhension que Gao a de Nietzsche reste toujours dans l'ombre portée des intellectuels des années 1980.

73. GAO, X. J., «Le modernisme retardé et la littérature chinoise actuelle », dans Littérature chinoise, op. cit., p. 59-60. 
Gao n'approuve pas non plus l'attitude anti-tradition de l'époque du 4 mai, ni les critiques radicales de son époque, comme celle de Liu Xiaobo ${ }^{74}$, le futur lauréat du prix Nobel de la paix. Il reconnaît au contraire l'impossibilité de déraciner la tradition:

Critiquer ce qui a été critiqué et ainsi de suite, ressemble à une fleur diabolique qui ne porte pas de fruits, il est donc du devoir de la littérature chinoise de s'en dégager, une fois pour toutes [...] Quand on discute de la façon dont la littérature chinoise doit se conformer à son temps, il est inévitable de prendre position vis-à-vis de la culture traditionnelle [...] Certes, la modernisation de la littérature chinoise contemporaine ne peut éviter de se baser sur sa culture traditionnelle nationale ${ }^{75}$.

Néanmoins, la tradition que Gao met en exergue n'est pas la tradition du « mainstream " politisée, mais celle d'une culture originelle, marginale et a-politique:

Né dans le bassin du fleuve Jaune et considéré comme la culture traditionnelle orthodoxe de Chine, le rationalisme confucéen n'est qu'une idéologie politique et éthique qui a contribué largement à la consolidation et à la prospérité des empires féodaux chinois, tout en recréant toujours le développement de la littérature et de l'art [...] La prospérité de la littérature et de l'art dans l'histoire chinoise a pris ses sources principales dans d'autres domaines de la culture chinoise: la philosophie taoïste de la nature ainsi que la culture primitive et folklorique qui s'étend du sud au nord de notre territoire et à travers toute l'histoire de la nation chinoise. Le bassin du fleuve Bleu constitue le plus grand berceau de cette culture. Par ailleurs, en amont du fleuve Jaune, du golfe Bohai jusqu'aux régions littorales de la mer Orientale, dans les contrées des minorités nationales du Sud-Ouest, du Nord-Ouest et du Nord-Est, la culture régionale ou minoritaire a beaucoup contribué à la culture de la nation chinoise. J'appelle cette dernière culture la culture non lettrée. En dehors de tout cela, la religion, en tant qu'élément composant de l'idéologie, est toujours inséparable de la culture traditionnelle d'une nation; tout comme le christianisme l'est de la culture européenne, l'islam, de celle des Arabes, le bouddhisme, de celle de l'Inde. Le taoïsme, né à

74. Par exemple: LIU X. B., 《危机, 新时期文学面临危机》Weiji, xinshiqi wenxue mianlin weiji ("Crise, la littérature de la nouvelle époque envisage la crise »), dans «深 圳青年报》Shenzhen qinnianbao (Journal de la jeunesse de Shenzhen) du 2 octobre 1986.

75. GAO, X. J., « Le modernisme retardé et la littérature chinoise actuelle », dans Littérature chinoise, op. cit., p. 60 
l'intérieur de la Chine et le bouddhisme introduit de l'Inde ont tous deux profondément influencé la formation de la culture traditionnelle chinoise. La doctrine mystérieuse liée à la philosophie taoïste et à l'école du Dhyâna a exercé une influence imperceptible sur la psychologie esthétique des fonctionnaires-lettrés de l'ancienne Chine et de nos intellectuels d'aujourd'hui. Elles-mêmes sont ainsi devenues un mode de sensation et de pensée très originales ${ }^{76}$.

Une telle déclaration vantant une nouvelle « origine " culturelle est sans doute la conséquence de son long voyage dans le sud-ouest de la Chine en 1983-1984. La pièce de théâtre L'Homme sauvage (1984) est un exemple d'application de cette idée d'exploiter la source de la culture folklorique. Un écologiste quitte sa femme à la ville et vient dans la forêt de Linqu pour aller sur les traces de l'homme sauvage. Il rencontre donc toutes sortes de personnes qui s'intéressent à l'homme sauvage ou qui y cherchent leurs intérêts: le journaliste, l'explorateur, le scientifique et les braconniers. Au fur et à mesure que se déploie son enquête, cet écologiste en vient à douter que l'homme sauvage existe vraiment, le seul témoin du fait n'étant qu'un petit garçon. En témoignant de la destruction par la civilisation moderne du système écologique ainsi que de la disparition de la civilisation sauvage liée à la nature, il se rend compte que l'homme sauvage n'est que la représentation de leurs convoitises, y compris la sienne. À la fin de la pièce, l'homme sauvage apparaît dans le rêve du petit garçon et communique avec lui via un « langage » naturel.

Il y a dans cette pièce de théâtre une sorte de regard lyrique sur la civilisation primitive et, en même temps, une légère tristesse quant à ce retour impossible. Mais ce qui est le plus remarquable est la découverte de la culture de $\mathrm{Chu}^{77}$, aussi ancienne que la culture de $\operatorname{Han}^{78}$, mais ignorée depuis longtemps dans la tradition " mainstream ». Gao atteste de la persistance de la culture de Chu à travers les anciens chants, tel La légende des ténèbres, et aussi la vie quotidienne de villageois. Henry Zhao disait qu'il y a « un esprit libre sans aucune entrave " et « un enthousiasme à entrer dans le fond

76. Ibid., p. 61

77. 楚文化, la culture régionale ou minoritaire et taoïste, née dans le bassin du fleuve Bleu.

78. 汉文化, la culture centrale ou confucianiste née dans le bassin du fleuve Jaune. 
de la culture ${ }^{79}$ ". L'ambition de Gao ne s'arrête pas là: il ne veut pas seulement découvrir la culture de $\mathrm{Chu}$, mais aussi la reconstruire et la revaloriser comme une culture aussi originelle et féconde que la culture Han. Dès lors, la culture Chu devient l'une des sources les plus inspirantes de sa vie littéraire: du roman (La Montagne de l'âme) aux pièces de théâtre (Classiques des montagnes et des mers, Cité d'enfer,...) et aussi à l'opéra (La Neige en août).

En ce sens, Gao se rapproche - tout en gardant ses distances de "la littérature qui recherche ses racines (寻根文学)", comme en témoigne en 1985 son texte ainsi dénommé par allusion au titre d'un article de Han Shaogong (韩少功), « Les racines de la littérature " paru la même année ${ }^{80}$. Il ne s'agit pas d'un courant organisé sous un slogan littéraire ou politique, mais plutôt d'un phénomène littéraire représenté par des romans de la même tendance, paraissant presque simultanément en Chine, du nord au sud, de l'est à l'ouest. À la manière de l'allégorie et du symbolisme, les écrivains exploitent l'inconscient archaïque et l'inconscient collectif dans le contexte de la culture originelle. Par exemple, Le village de Bao (“小鲍庄 》) de Wang Anyi (王安忆) réinterprète la notion d'humanité (Ren, 仁) à travers l'histoire d'un petit garçon qui est le signe de Ren; alors que dans $B a B a B a$ de Han Shaogong, la critique ne porte pas seulement sur la culture de Chu mais aussi sur toute la culture chinoise: le protagoniste Bingzai, le petit idiot immortel est le signe symbolique d'une culture appesantie par une inertie " éternelle ", qui stagne dans le même endroit, s'y enferme, reste insensible au temps qui passe et répète le même mode de pensée de génération en génération. "La littérature qui recherche ses racines " propose ainsi des points de vue variés qui sont les bienvenus pour réexaminer autrement la culture chinoise, mais, en même temps, elle témoigne du choc ressenti à envisager de nouveau ses propres cultures et révèle, a contrario, la force du désir de poursuivre de la modernisation.

79. ZHAO, H., Erya (尔雅), 2001, p. 89. Voir aussi sa version anglaise Towards a Modern Zen Theatre, London, School of Oriental and African Studies, 2000, p. 82.

80. HAN, S. G., « Les racines de la littérature 》 ( «文学的根 》), dans Écrivain ( 《作家 »), $1985, n^{\circ} 4$. 
Chen Xiaoming (陈晓明), chercheur en littérature chinoise contemporaine, le montre bien, ce courant littéraire est avant tout de la littérature de zhiqing envoyés à la campagne ${ }^{81}$. La plupart des écrivains de ce courant littéraire partagent la même expérience: ils ont dû quitter la ville - l'univers modernisé/occidentalisé - et se sont retrouvés à vivre à la campagne où le poids mais aussi les séductions de la culture locale de cette Chine " authentique " ne pouvaient qu'accentuer le contraste avec l'Occident de référence chez les intellectuels.

Le choc entre deux cultures et aussi entre la réalité et l'illusion, la réflexion sur les origines culturelles mais aussi le désir de la modernisation sont les réactions les plus directes peut-être, et instinctives de ces " exilés ». Ainsi, conclut Chen:

[...] dans la littérature qui recherche ses racines, la mémoire personnelle de zhiqing est agrandie jusqu'à la mémoire du collectif, de l'époque et de la nation, ainsi la narration du souvenir personnel se transforme en narration de l'histoire de la nation [...] à partir de ce point de vue, les écrivains de «la littérature qui recherche ses racines » ont raison de se prétendre les sujets de la nation/histoire et les porte-parole de la culture du «tiers monde » dialoguant avec la culture occidentale ${ }^{82}$.

Pour Gao, la rencontre de l'Occident et de la tradition ne constitue pas un choc culturel: elle n'est qu'une expérience personnelle qui ne donne pas lieu à des jugements de valeur et même à la redécouverte des mémoires collectives. Voici comment il présente son positionnement entre les deux cultures: "J'estime plus convenable de m’en tenir à la croisée des cultures oriento-occidentales et à celle du passé et d'aujourd'hui ${ }^{83}$.»

81. Les «Zhiqing à la campagne (知青下乡) » désignent un ensemble de douze à dixhuit millions de jeunes Chinois envoyés des villes à la campagne depuis les années 1950 jusqu'à la fin de la révolution culturelle, suite à une initiative de Mao. Environ la moitié de la génération des jeunes urbains fut concernée. Ce mouvement a débouché sur une crise de société, les jeunes voulant à tout prix retourner à la ville. On en parle souvent comme d'une " génération perdue ». Gao a été personnellement pris dans cette tourmente.

82. CHEN, X. M., 《中国当代文学主潮 》 (Les courants littéraires de la littérature chinoise contemporaine), Beijing, Beijing daxue chubanshe, 2009; voir le chapitre 13.

83. GAO, X. J., « Le Modernisme retardé et la littérature chinoise actuelle », dans Littérature chinoise, op. cit., p. 62. 
Mais, comment se positionne-t-il entre l'Orient et l'Occident, notamment lorsqu'il se trouve pris dans une tradition où l'esthétique s'incorpore à l'éthique? Fondamentalement, la modernité chinoise et la modernité occidentale sont-elles compatibles?

\section{Auteur apolitique et politisé, moderne et héritant de la tradition}

Les ouvrages de Gao, dans les années 1980, s'associent souvent aux nouveaux courants littéraires apparaissant à l'époque, mais s'en distinguent en même temps: comme Étoile dans la nuit froide à « la littérature des cicatrices ", Premier essai sur la technique du roman moderne et ses nouvelles, ainsi que sa pièce de théâtre et L'Arrêt d'autobus à "l'école moderne", L'Homme sauvage à "la littérature qui recherche des racines ». Gao refuse d'être classé dans un quelconque courant: "Je n’ai jamais approuvé l'appellation "école moderne" qui m’a été imposée; ni ne suis prêt à figurer dans le rang des partisans de "la recherche des racines" 84 " .

Si Gao refuse d'être étiqueté, c'est sans doute, tout d'abord, de par sa volonté de garder son indépendance - de ne pas être submergé par la voix collective ni prétendre la représenter. C'est aussi parce que sa conception de la littérature se différencie tant de la littérature traditionnelle comme " véhicule de la Voie " (文以载道), que de celle du $x^{e}$ siècle comme outil de la propagande politique.

De fait, une tendance résolue à l'apolitisme et à l'individualisme, deux caractéristiques essentielles de Gao, le distingue des autres écrivains chinois de son temps. Cette même tendance le conduit à un paradoxe. L'une des idées qui animent les ouvrages de Gao est qu'il importe de combattre l'idéologie et l'éthique, deux maux dont il tente de se détacher. Notamment, dans son dernier roman, Le livre d'un homme seul (1999), la politique se présente comme un métaterme auquel il résiste sans cesse mais qui l'obsède toujours: il y va de la confrontation entre l'individu et le collectif, entre le pouvoir de la foule et le pouvoir de l'individu, entre la libération personnelle et la responsabilité pour le pays et la nation.

84. Ibid. 
Chen Xiaoming soutient, non sans pertinence, que l'apolitisme de Gao l'a politisé malgré lui:

Son caractère politique est représenté justement par son effort de se débarrasser d'entraves politiques, et sa recherche d'une manière personnelle d'exprimer ce qui s'enracine dans le fond de son esprit: cela est une politique étrange. C'est aussi le reflet de la littérature chinoise politisée $e^{85}$.

Gao a beaucoup parlé de Lu Xun, qui fait particulièrement référence à ses yeux. D'un côté, il le considère comme l'un des rares écrivains chinois modernistes; de l'autre, Gao critique l'engagement de Lu Xun dans la politique; il y gaspille, pense-t-il, son talent littéraire: "l'écrivain Lu Xun est étouffé par le politique Lu Xun ». En fait Lu Xun est tout simplement comme lui-même (au moins durant les années 1976-1999): contraint de se débattre dans la contradiction entre la politique et la littérature.

Par ailleurs, Gao ne souscrit pas à l'antitraditionalisme de $\mathrm{Lu}$ Xun:

Le mouvement du 4 mai a déjà critiqué la culture féodale. Pas un de nos contemporains n'a pu le faire aussi radicalement que Lu Xun. Or, ce que Lu Xun n'avait pas la possibilité de faire ou d'achever, c'était d'envisager cette culture traditionnelle avec un œil contemporain ${ }^{86}$.

Mais peut-être, de ce point de vue, Gao soupçonne-t-il entre eux deux une égale sensibilité à la modernité, amenée à s'exprimer sous des modalités contradictoires. Il s'agit toujours d'" envisager cette culture traditionnelle avec un œil contemporain ». Si Lu Xun a réagi par l'antitraditionalisme à l'impact des idées occidentales modernes au début du $\mathrm{xx}^{\mathrm{e}}$ siècle, lancer un regard nouveau sur la culture chinoise est la réponse détournée de Gao, via la tradition, au déferlement du modernisme. En d'autres termes, l'influence occidentale se marque chez les deux écrivains, s'agissant de " retour " vers la tradition, de deux façons opposées: la réinterpréter ou la contrecarrer.

85. GAO, X. J., 《从现代主义到没有主义 》 («Du modernisme à ne pas avoir d'-isme »), dans «二十一世纪》 Ershiyi shiji (Twenty-first Century), 2000, n 62, p. 34.

86. GAO, X. J., « Le modernisme retardé et la littérature chinoise actuelle », dans Littérature chinoise, op. cit., p. 60 
Bien que l'Occident et la tradition revêtent des connotations différentes chez Lu Xun et chez Gao, et qu'ils les influencent différemment, les deux écrivains et aussi penseurs, en tant que les plus sensibles et plus subtils modernistes de leurs époques respectives, celle du 4 mai et celle des années 1980, font apparaître une filiation de l'un à l'autre par rapport à la modernité - ou comme une sorte de multi modernité s'opposant parfois à elle-même.

\section{LE PARADOXE DU MOI CHEZ GAO}

\section{La fuite}

La pièce de théâtre La Fuite, écrite en 1989, raconte l'histoire qui se déroule dans la nuit de la place Tian'anmen (4 juin) entre trois personnages qui ne se connaissent point mais qui ont tous participé à ce mouvement. Gao a subi immédiatement des critiques, à la fois du côté des autorités chinoises et du côté du mouvement démocratique $^{87}$. Car le dramaturge ne souscrit ni à la répression de la dictature ni au sacrifice d'individus pour le pays au nom de la démocratie. Cette pièce qui touche un sujet si sensible, de la politique et de la réalité, apparaît avant tout comme une déclaration personnelle de Gao: chacun n'est le responsable que de lui-même et non le responsable d'un «nous " brandi au nom du pays, de la nation ou de la volonté collective.

La Fuite commence par montrer deux jeunes, un homme et une fille qui fuient la place et se cachent dans un entrepôt désaffecté. Puis un homme d'âge mûr, qui s'est enfui de son appartement pour échapper à la police et ne pas être arrêté devant sa fille, s'abrite dans ce même endroit. Ils échangent quelques mots sur la situation: un conflit surgit tout de suite entre le jeune homme et l'homme d'âge mûr. Celui-ci regrette que les étudiants et les citoyens, y compris luimême, n'aient pas pensé à organiser la retraite avant que les armes n'entrent en action et restent sans rien faire devant la répression — il s'agit d'« un véritable suicide... héroïque mais naïf et stupide ».

87. Cette pièce fut commandée par un théâtre américain : lorsque ce dernier a demandé à Gao de la modifier, sans doute pour raison de political correctness, Gao a refusé tout net et l'a retirée. 
Tandis que le jeune homme tient à croire que c'est le prix nécessaire à payer pour pousser le processus démocratique du pays:

Le jeune homme: La lutte du peuple pour obtenir sa liberté triomphera tôt ou tard. Même si nous devons payer le prix du sang. L'homme: Et pourquoi, dans ce cas, as-tu fui devant les chars? Le jeune homme: Je ne voulais pas d'un sacrifice inutile. L'homme: Mais qui donc fera ce genre de sacrifice alors? Des gens comme moi peut-être? Tu parles au nom du peuple mais tu ne sais pas te diriger toi-même. Tu parles de victoire finale mais tu ne parviens pas à te vaincre toi-même. Cette lutte pour la liberté n'entraînera que la mort: elle équivaut au suicide. S'il n’y a plus de vie, quel sens peut encore avoir une hypothétique victoire finale? La vérité, c'est que toi et moi devons fuir ${ }^{88}$ !

Il s'agit davantage d'une discussion sur la philosophie de chacun que sur la politique. Les deux personnages s'affrontent sur la question $\mathrm{du}$ " moi » et du « nous ». Le jeune homme représente un moi romantique et héroïque auquel l'homme d’âge mûr renonce. Ce dernier ne veut s'engager ni dans le mouvement ni dans l'idéologie. La fuite, pour lui, n'est pas une action lâche, au contraire, c'est une rébellion, une façon de sauvegarder la volonté individuelle indépendante face à la volonté collective:

L'homme: Sans doute. Mais je suis suffisamment conscient pour refuser d'être joué par d'autres. Je compte sur ma propre volonté, immuable et indépendante. C'est pourquoi... je fuis!

Le jeune homme (calme et haineux): En fait, tu nous fuis aussi et tu fuis le mouvement démocratique.

L'homme: Je fuis toute prétendue volonté collective.

Le jeune homme: Si tout le monde était comme toi, ce pays n'aurait plus d'espoir.

L'homme: Quel pays? Le pays de qui? Ce pays a-t-il assumé ses responsabilités envers toi et moi? Pourquoi devrais-je être responsable de lui? Je ne suis responsable que de moi-même.

Le jeune homme: Et tu peux regarder l'anéantissement de notre nation sans sourciller?

L'homme: Je n'ai qu'un but: me sauver moi-même. Si un jour cette nation doit s'éteindre, tant pis: qu'elle s'éteigne!... Tu as d'autres questions? Ou l'interrogatoire est terminé?

Le jeune homme (hagard): Tu es un...

88. GAO, X. J., Gao Xingjian théâtre 1, Belgique, Lansman, 2000, p. 15. 
L'homme: Un individualiste? Un nihiliste? Je ne suis aucun "iste”, et je n'ai pas besoin de croire en un "isme” quelconque. Je suis un être vivant, qui ne peut supporter d'être massacré... ou poussé au suicide ${ }^{89}$.

$\mathrm{Hu}$ Yaoheng, ami de Gao et professeur en théâtre à Taiwan, remarque que cet homme d'âge mûr ressemble aux personnages de Brecht (ceux de Mère courage et ses enfants, par exemple), puisqu'ils sont tous les "héros d'anti-héros ${ }^{90}$ ". Henry Zhao estime que la déclaration de cet homme doit être comprise comme la plaidoirie pour l'exil de Gao lui-même ${ }^{91}$. Le personnage, pour autant qu'il est la figure de l'auteur, révèle la complexité de celui-ci: via ce personnage, Gao se représente dans le mouvement de Tian'anmen dont il était absent, et par sa bouche exprime son propre désir de fuir. La fuite n'est-elle pas le propre de la destinée humaine? " C’est à la fuite que nous sommes confrontés, c'est notre destinée, à toi et à moi. (En murmurant) La destinée humaine, c'est perpétuellement la fuite ${ }^{92}$ ! "

Mais cette fuite n’implique-t-elle que le désir d'échapper à la politique, à l'éthique ou à la volonté collective? Il y a une scène où la jeune fille et le jeune homme parlent, synchroniquement et parallèlement, pour exprimer leur ressenti de la répression de cette nuit:

La jeune fille: Je regarde tomber de gros flocons, de gros flocons de neige. Ils font disparaître complètement la place et les rues. Comme un grand linceul, ils recouvrent la ville.

Le jeune homme (sur un ton parodique): Je vois un soleil noir. Une lumière blême éclaire les familles endeuillées.

La jeune fille (obstinée): Je regarde tomber de gros flocons, de gros flocons de neige. La ville est blanche et silencieuse...

Le jeune homme: Tous restent debout sur la place blanche où se déroule l'oraison funèbre en hommage aux martyrs innombrables sacrifiés dans un lac de sang.

89. Ibid., p. 28-29.

90. Hu, Y. H. (胡耀恒), 《百年耕耘的丰收-高行健戏剧六种》, Taibei, Dijiao Chubanshe ( 帝教出版社), 1996, p. 36-37.

91. ZHAO, H., 《高行健与中国实验戏剧-建立一种现代禅剧》, Taibei, Erya, 2001, p. 106. Aussi dans Towards A Modern Zen Theatre. Gao Xingjian and Chinese Theatre Experimentalism, London, School of Oriental \& African Studies, 2000, p. 97.

92. GAO, X. J., Gao Xingjian théâtre 1, op. cit., p. 16. 
La jeune fille: Dans ce monde tout blanc, nous grimpons sur un mur en ruine couvert d'herbes sauvages. L'air est pur et glacial. De gros flocons, de gros flocons de neige tombent...

Le jeune homme (entrant progressivement dans le jeu): Je me relèverai de ce fleuve de sang. Je veux vivre jusqu’à ce jour!

La jeune fille: Tu me tiens par la main et nous grimpons sur le mur détruit. La neige recouvre les rues.

[...]

Le jeune homme: Nous allons fuir cette obscurité. J'ai toujours rêvé de ce jour où aura lieu la grande cérémonie commémorative en l'honneur de ceux qui se seront sacrifiés pour le pays...

[...]

La jeune fille: Je dois être élégante pour paraître lors de ces soirées de fête. (Au bord de la flaque, elle allume le briquet qu'elle tend à deux mains) Je veux voir mon corps sur la surface du miroir ${ }^{93}$.

Comme une manière d'incitation (xing, 兴), la neige provoque une fuite sans fin qui pousse la fille à s'échapper de la place et de la ville, et la fait aller jusqu'au mur en ruine. Ainsi, en suivant la neige, le ressenti de la jeune fille dérive du mouvement démocratique aux souvenirs et à l'imagination amoureuse. La neige est présente dans presque tous les œuvres de Gao: La Montagne de l'âme, Le Livre d'un homme seul, L'Autre Rive, Au bord de la vie, La Neige en août, Quatre quatuors pour un week-end... Par la grâce de la neige qui est cristallisation de l'eau et qui peut se transformer librement en solide, en liquide ou en gaz, Gao exprime son éloge et son désir de la fuite.

La neige emblématise une fuite qui échappe au monde extérieur et s’opère dans le monde intérieur. Dans cette pièce de théâtre, la fille est amenée par la neige à entrer dans ses souvenirs et dans son imagination, tandis que le jeune homme, qui ne dialogue pas réellement avec elle, tient un discours parallèle et est entraîné finalement par son propre discours: il se tourne aussi vers son monde intérieur. Puis l'homme d'âge mûr les rejoint:

La jeune fille: J'aimerais qu'un miroir couvre tous les murs afin de pouvoir m'admirer et de voir tomber les gros flocons, les gros flocons de neige. Je marche dans la neige, Je mets mon cops à nu. Mon corps et mon âme sont transparents comme après l'amour, dans un instant radieux où je m'oublie totalement.

93. Ibid., p. 37 . 
Le jeune homme: Je suis assis au bord d'une mer déferlante et je regarde l'océan à perte de vue. Se succédant comme des chaînes de montagne, les vagues se brisent sur la grève, sous un ciel gris-acier. Une traînée d'écume blanche bondit sur le noir des vagues.

L'homme: Tu marches au milieu de fleuve des morts, gardant cette petite lueur obscure dans le cœur. Je continue à voir cette image comme dans mon rêve. En fait, je n'arrive plus à distinguer si c'est un rêve, un mirage ou une simple sensation ${ }^{94}$.

Pour autant, le monde intérieur n'est pas le refuge du moi. Les trois personnages ont beau se plonger chacun dans son univers intime, le moi est ce dont on ne peut jamais s'échapper. La neige, faisant image, le révèle aussitôt : chez Gao, elle apparaît souvent lorsque le personnage se torture lui-même ${ }^{95}$.

Ainsi dans La Fuite, la jeune fille révèle ce secret du moi lorsque l'homme d'âge mûr a envie de partir: au moment où la fille console le jeune homme:

L'homme: Non, ne recommençons pas. Je veux fuir... me fuir moimême.

La jeune fille: Alors ouvre la porte, pars et laisse-nous! Aurais-tu peur de la mort, toi aussi?

L'homme (hésitant): Je ne crains pas particulièrement la mort. Ce qui m'effraie, c'est le désespoir... le désespoir avant la mort, qui pousse les gens à s'entredéchirer dans une sorte de danse hystérique.

La jeune fille: Ce qui te fait peur, c'est toi-même! Tu peux fuir n'importe où: jamais tu ne pourras échapper à toi-même ${ }^{96}$ !

C'est la raison pour laquelle Gao déclare que l'enfer, c'est le moi:

Fuir, c'est se sauver soi-même, mais le plus difficile est sans doute de fuir les ombres que l'on porte en soi; si l'on n'a pas de perception claire du moi, on risque de se perdre dans son propre enfer et de ne jamais voir le jour jusqu'à sa mort. Cet enfer intérieur, ce sont les illusions qui étouffent les hommes et les conduisent à la destruction ${ }^{97}$.

94. Ibid., p. 38-39.

95. Par exemple, à la fin d'Au bord de la vie, quand la femme est épuisée par sa propre interrogation sur le moi, la neige apparaît comme une réponse : " (Le vieillard s'arrête devant la pierre, s'appuyant sur la canne, comme pour s'asseoir, mais hésite, en regardant voltiger un flocon de neige, invisible en fait, qui tombe finalement juste devant ses pieds. II enlève son chapeau, fait semblant de recevoir de la neige) », voir GAO, X. J., Gao Xingjian théâtre 1, op. cit., p. 85.

96. GAO, X. J., Gao Xingjian théâtre 1, op. cit., p. 55.

97. GAO, X. J., «Littérature et liberté », dans De la création, traduit par Noël Dutrait, Paris, Seuil, 2013, p. 305. 
Gao, qui lutte contre toutes les idéologies politiques, contre la volonté collective afin de garder la liberté et l'indépendance de la subjectivité individuelle en se détachant de la responsabilité et en sortant de l'enthousiasme du moi héroïsé, se trouve désormais face à ce moi qui, si précieux et si cher jadis, n’est en réalité qu'un monstre-insecte kafkaïen, solitaire et désemparé: le moi " chaos ", l'appelle-t-il.

Bien que la subjectivité individuelle chez Gao se trouve avoir des ressemblances avec la subjectivité du modernisme occidental, elle s'en distingue non seulement en raison des singularités personnelles, mais du fait de la pensée et de la culture qui en sont le soubassement.

Lorsque la littérature moderniste occidentale se gausse de la valeur de l'être humain, de la raison, de la religion, de tout ce qui était considéré comme solide et véritable, elle se focalise sur le « moi », car le subjectivisme occidental n'échappe pas au moi, même lorsqu'il l'évide et le torture. L'inconscient freudien, la dérision, l'ironie et la dévalorisation à l'égard de l'existence n'impliquent pourtant pas l'ignorance de l'Être: c'est encore et toujours le « moi ", cet indéracinable moi qui perdure en changeant la direction de son regard. C'est-là le piège du " moi » qui ne peut que s'enfoncer dans l'ontologie omniprésente: l'Être sans fin.

\section{Entre le moi chaos et le moi qui ne s'appuie ni sur le subjectivisme ni sur l'objectivisme}

Si la modernité occidentale est fondée sur " une conscience du nouveau " par rapport aux concepts de raison et de subjectivité individuelle ${ }^{98}$ issus du propre fonds européen (judéo-chrétien, philosophique, etc.), la modernité chinoise du $\mathrm{xx}^{\mathrm{e}}$ siècle quant à elle se fonde plutôt sur l'irruption de la nouveauté dans l'éthique/politique et la subjectivité collective. Alors, qu'est-ce " qui se tient au-dessous » de la modernité chez Gao? Comment interprète-t-il la notion de subjectivité/moi dans ses ouvrages?

98. HABERMAS, J., Le Discours philosophique de la modernité, traduit de l'allemand par Rainer Rochlitz et Christian Bouchindhomme, Paris, Gallimard, 1988, p. 1-26. 
Habermas souligne que Hegel fut "le premier à ériger en problème philosophique la rupture de la modernité avec les suggestions normatives du passée ${ }^{99}$ ", pour avoir découvert le principe du temps moderne: la subjectivité. Hegel explicite la subjectivité par la " liberté » et par la "réflexion »: "Ce qui fait la grandeur de notre temps, c'est que l'on a reconnu la liberté, la propriété de l'esprit, le fait qu'il est en soi auprès de soi $^{100}$. » Selon Habermas, les événements historiques clefs qui ont imposé ce principe de la subjectivité sont "la Réforme, les Lumières et la Révolution française ": par la Réforme, "la foi religieuse devient réflexive " — dans la solitude de la subjectivité, « le monde divin s'est changé en une réalité instaurée par nous-mêmes "; les Lumières et la Révolution proclament les droits de l'homme et le code Napoléon impose le principe de liberté ${ }^{101}$.

En référence sans doute à ce contexte, Habermas énonce que le vrai visage de la raison est " une subjectivité qui assujettit tout en étant elle-même asservie ${ }^{102}$ ». De même, la structure de la subjectivité adhère à la logique de la connaissance:

Sa structure en tant que telle est saisie dans la philosophie, à savoir comme subjectivité abstraite dans le cogito ergo sum de Descartes et dans la figure de la conscience de soi absolue chez Kant. Il s'agit là de la structure propre au rapport à soi du sujet connaissant qui se penche sur lui-même en tant qu'objet, afin de se saisir comme dans une image spéculaire, précisément de manière « spéculative » ${ }^{103}$.

Mais, se demande Habermas,

si le principe de la subjectivité et la structure de la conscience de soi qui lui est inhérente sont des sources suffisantes pour satisfaire le besoin d'orientations normatives; si elles sont suffisantes autrement dit, non seulement pour "fonder ", d’une façon générale, la science, la morale et l'art, mais pour stabiliser une formation historique qui s'est émancipée de toute obligation historique. Dès lors se pose la question suivante: est-il possible de tirer de la subjectivité et

99. Ibid., p. 18.

100. Ibid., p. 19

101. Ibid., p. 20

102. Ibid., p. 5.

103. Ibid., p. 21-22. 
de la conscience de soi des critères empruntés au monde moderne et en même temps aptes à permettre de s'orienter dans ce monde, ce qui veut dire aussi: aptes à critiquer une modernité en lutte avec elle-même. Comment peut-on, à partir de l'esprit de la modernité, construire une figure intérieure idéale qui ne se contente pas d'imiter les multiples formes historiques sous lesquelles la modernité est apparue, ni de s'imposer à ces formes d'un point de vue extérieur ${ }^{104}$ ?

Les interrogations d'Habermas viennent sans doute de l'autoréférence d'un sujet qui se fait lui-même objet et qui cherche à se connaître mais, en même temps, se réfère à lui-même: dans cette connaissance de l'autonomie du sujet lui-même où la subjectivité révèle inévitablement qu'elle n’est qu' « un principe unilatéral ${ }^{105}$ » et qu'elle " perd la force de produire spontanément le monde ${ }^{106}$ ».

Michel Foucault, quant à lui, renonce à cette subjectivité — plus précisément, à l'autoréférence et à l'autonomie du sujet - en rompant avec le moi héroïsé, et aussi avec les Lumières. Mais, aux yeux de Habermas, ce n'est qu'une autre impasse:

L'embarras dans lequel la philosophie du sujet plonge les sciences humaines est critiqué de manière lumineuse par Foucault; il montre bien comment, en fuyant l'aporétique à laquelle conduisent les autothématisations contradictoires d'un sujet qui entreprend de se connaître lui-même, elles ne font que s'enfoncer un peu plus dans un scientisme autoréifiant ${ }^{107}$. [Car] à l'objectivisme de la maîtrise de soi, là, correspond, ici, le subjectivisme de l'oubli de soi ${ }^{108}$.

Mais y a-t-il une autre manière de connaître le moi, qui ne soit engluée ni dans le subjectivisme ni dans l'objectivisme?

La division dualiste n'existe pas dans la pensée traditionnelle chinoise. Lorsque Gao remonte dans sa propre culture en parcourant une autre voie que celle du confucianisme, il y trouve une

104. Ibid., p. 23-24.

105. Ibid., p. 24.

106. Habermas, J., La Pensée postmétaphysique. Essais philosophiques, traduit de l'allemand par Rainer Rochlitz, Paris, Armand Colin, 1993, p. 247.

107. HABERMAS, J., Le Discours philosophique de la modernité, op. cit., p. 348.

108. Ibid. 
pensée originelle qui supporte sa notion de l' « individu » sans s’appuyer ni sur le subjectivisme ni sur l'objectivisme.

Gao construit sa notion de la subjectivité à l'aide du taoïsme et du bouddhisme chinois chán (禅), où le vrai moi se trouve dans l'état de non-moi. Cette pensée inspire d'emblée sa théorie de la « fuite " (隐逸). En effet, la fuite est aussi une tradition pour les lettrés chinois lorsqu'ils sont étouffés par la réalité politique: elle consiste à se retirer et à se réfugier dans la pensée taoïste et bouddhiste afin d'y chercher la liberté de l'individu. Zhuangzi appelle cela « accomplir le « $\operatorname{corps}^{109}$ » et prendre soin de la vie (完身养生) ", c'est une valeur primordiale qui l'emporte sur la force du règne politique:

L’essence du Dao sert à préserver la personne, ses restes à s’occuper des affaires du pays, ses déjections à gouverner l'empire. À ce point de vue, la tâche du souverain et du roi ne représente que le travail accessoire de la sagesse, elle ne contribue en rien à l'accomplissement de la personne et à prendre soin de la vie. Alors que les gens de nos jours sont prêts à sacrifier leur vie et leur personne pour les choses extérieures. N'est-ce pas lamentable ${ }^{110}$ ?

Ainsi, cette liberté se fonde sur l'oubli du soi. Chez Laozi et Zhuangzi aussi bien que dans le bouddhisme chinois chán, l'obstacle le plus grand à « la disponibilité/liberté totale ${ }^{111}$ (zizai, 自在)", est le

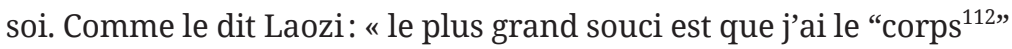
(贵大患若有身)», et c'est pourquoi « l'homme à l'état supérieur est

109. II ne s'agit pas du corps qui est opposé à l'âme comme dans le dualisme « corps/ âme », mais d'un « corps » qui n'est plus conçu comme une substance matérielle.

110. En chinois: «道之真, 以治身, 其绪余以为国家, 其土苴以治天下。由 此 观之, 帝王之功, 圣人之余事也, 非所以完身养生也。今世俗之君子, 多危 身弃生以殉物, 岂不悲哉。»（《庄子 - 让王»）, Zhangzi, chapitre XXVIII; nous le traduisons en comparant la traduction de Jean Levi (Les E Fuvres de maitre Tchouang, Paris, Paris, Édition de l'Encyclopédie des nuisances, 2006) et de Liou Kia-hway (L'É Euvre complète de Tchouang-tseu, Paris, Gallimard, 1969).

111. François Jullien traduit cette "liberté totale (zizai) » par « disponibilité », une disponibilité qui concerne « prioritairement des biens, des avoirs ou des fonctions. Elle n'a guère pris de consistance, en revanche, du côté de la personne ou du sujet [...] un sujet qui dès l'abord présume et projette, choisit, décide, se fixe des fins et s'en donne les moyens »: voir dans Cinq concepts proposés à la psychanalyse, Paris, Grasset, 2012, p. 23.

112. LAOZI, «老子道德经注校释(王貆注) » Laozi Daodejing, Beijing, Zhnghua shuju, 2008, p. 28. 
sans soi ${ }^{113}$ (至人无己). Pour atteindre ce zizai qui s'incarne dans le Dao chez les taoïstes et dans la vacuité (空) chez les bouddhistes, il faudrait se détacher de soi-même - en autres termes, " renoncer à son initiative de "sujet ${ }^{114}$ " ".

Le moi chez Gao est complexe, écartelé entre les deux cultures. Pour lui, la modernité commence par la remise en cause du « moi ", celui par lequel la crise moderne a été provoquée:

La littérature moderne laisse de côté les questions éthiques du bien et du mal qui, à l'origine, ne faisaient aucun doute, tandis que l'analyse du moi conduit à la schizophrénie ${ }^{115}$.

Le « moi » douteux est donc le point clé chez Gao, comme chez la majorité des écrivains modernes. Mais en même temps, la notion du moi est influencée par la pensée taoïste et bouddhiste: il s'agit du renoncement à l'engagement politique, du détachement vis-à-vis de la volonté collective, et, en somme, de se trouver en marge de la société. C'est la raison pour laquelle, en parlant du moi-chaos, Gao n'éprouve ni la panique ni la perplexité, ni non plus l'incapacité de connaître ce moi obscur.

\section{Le non-isme et le paradoxe de la liberté de l'individu}

Chez Gao, la liberté de l'individu ou de la subjectivité ne s'incarne nulle part dans des conditions extérieures ni dans des connotations intérieures ${ }^{116}$. Tel est son principe de la liberté de l'individu: " ne pas avoir de-isme » ou " non-isme»:

113. ZHUANGZI, 《庄子集释(郭慶藩撰)》, Zhuangzi Jizhu, Beijing, Zhnghua shuju, 2004, p. 17 ; nous prenons la traduction d'A. C. Graham, Chuang-tzu. The Inner Chapters, London, Boston, Allen \& Unwin, 1981, p. 45.

114. JULLIEN, F., Cinq concepts proposés à la psychanalyse, op. cit., p. 25.

115. GAO, X. J., " Ne pas avoir de -isme », dans Le Témoignage de la littérature, op. cit., p. 36.

116. Comparons avec les connotations que Hegel propose pour accompagner le terme de subjectivité, en tant que fondé sur la liberté et la réflexion : a) I 'individualisme : dans le monde moderne, c'est la singularité infiniment particulière qui est en droit de faire valoir ses prétentions; b) le droit à la critique : le principe du monde moderne exige que ce que chacun doit accepter lui apparaisse comme quelque chose de justifié ; c) l'autonomie de l'action : il appartient aux temps modernes de vouloir répondre de ce que nous faisons ; 
Dans meiyou zhuyi, ce que j'appelle " ne pas avoir de-isme ", meiyou, "ne pas avoir", peut être considéré comme le groupe verbal, et zhuyi, "-isme ", comme un substantif, le tout formant une tournure indéfinie. Considérer " ne pas avoir de-isme " comme une construction verbe-complément, ou en faire une locution, wuzhuyi, "pas de -isme ", est aussi possible. Mais l'interpréter comme substantif, le "sans-isme ", risque d'être compris comme une sorte de -isme, au même titre que le nihilisme, et cela n'est pas adéquat.

Dans « ne pas avoir de -isme ", " ne pas avoir " constitue une condition préalable, et il ne faut pas faire du néant une condition préalable; sans condition préalable, il n'y aura naturellement pas de conclusion, et il n'y aura plus aucun -isme ${ }^{117}$.

Noël Dutrait croit que ce « ne pas avoir de -isme », n’est pas un concept philosophique, mais « une simple observation, guidée par le bon sens, qui témoigne d'une certaine posture face à l'activité humaine et politique ${ }^{118}$ ». Car la philosophie révèle d'emblée le système ou la construction de la pensée que Gao tente de contourner:

Ne pas avoir de -isme ne vise pas à construire une théorie, mais n’équivaut pas à se taire. Cela ne consiste qu'à parler sans point de départ ni point d'arrivée, à parler sans aboutir à aucune conclusion $^{119}$.

Cette déclaration n’évoque-t-elle pas une nostalgie des taoïstes, notamment dans le premier chapitre du Daode Jing ( Le Dao vraiment Dao est autre qu'un Dao constant $\left.{ }^{120} »\right)$ ?

Cependant, ne pas avoir de-isme ne revient pas à ne pas avoir d'opinion, à ne pas avoir de point de vue, à ne pas avoir de pensée, simplement, ce point de vue, cette opinion, cette pensée n'exigent aucune

d) enfin, la philosophie idéaliste elle-même: pour Hegel, c'est l'œuvre des temps modernes pour autant que la philosophie saisit l'Idée qui a conscience d'elle-même. Voir Le Discours philosophique de la modernité, op. cit., p. 20.

117. GAO, X. J., «Préface de l'auteur », dans «没有主义 » (Ne pas avoir de -isme), op. cit., recueillie et traduite par Noël et Liliane Dutrait, dans Le Témoignage de la littérature, op. cit., p. 7.

118. Dutrait, N., « Ne pas avoir de -isme », dans Perspectives chinoises, Hong Kong, 2010, $n^{\circ} 2$, p. 8-14.

119. «Préface de l'auteur », op. cit., p. 7-8.

120. LAOZi, 《老子道德经注校释(王貆注) » Laozi Daodejing, op. cit., p. 1. La traduction est empruntée à J. J. L. Duyvendak, Tao Tö King, le livre de la voie et de la vertu par Lao Tseu, Paris, Librairie d'Amérique \& d'Orient, 1953, p. 3. 
démonstration, aucun peaufinage, ne deviennent pas un système, on parle en vain - mais l'homme qui vit dans ce monde, excepté le muet, ne peut pas ne pas parler; c'est pourquoi la force de ne pas avoir de -isme n'est rien d'autre qu'un discours sans résultat ${ }^{121}$.

Il semble que ce soit un paradoxe d'affirmer, d'une part, que l' " on parle en vain » et, d'autre part, que l'on « ne peut pas ne pas parler ». Gao souligne quelques lignes plus loin que « ne pas avoir de -isme, c'est le droit élémentaire d'être un être humain » et " la condition minimale de la liberté individuelle de l'homme d'aujourd'hui ». Mais il n’est pas évident de déduire d’une " prémisse " plus ou moins taoïste, " ne pas avoir ", la conclusion: " avoir un certain droit »même s'il s'agit du droit d'être libre de toute contrainte. En ce sens, " ne pas avoir de -isme » ne se limite pas à « un discours sans résultat », comme Gao le prétendait.

Ce « ne pas avoir de -isme " semble proche du zizai - la disponibilité ou la « liberté totale » - , car les deux idées révèlent avant tout une attitude consistant à prendre de la distance par rapport à soi-même, afin de s'observer. Gao l'appelle « l'attitude de froide observation ", une attitude qui "vient de la tradition culturelle chinoise, les écrivains occidentaux étant davantage enclins à recourir à l'analyse psychologique et à l'expérience ${ }^{122}$ ". Pourtant, Gao constate aussi un paradoxe entre la disponibilité du « non-agir » et le moi qui veut avoir le droit de ne pas avoir de-isme:

Le « non-agir » de la philosophie de Laozi et de Zhuangzi, et la mise à l'écart du monde préconisée par le bouddhisme sont trop négatifs; tout compte fait, je veux bien, moi aussi, agir un peu, mais je ne suis ni taoïste ni bouddhiste, et je ne fais qu'adopter une attitude d'observateur $^{123}$.

Mais suffira-t-il d' "agir un peu " pour atteindre la disponibilité/liberté recherchée? Le paradoxe apparaît d'emblée dans le changement du point de départ: le moi fluide, qui ne délimite pas clairement la frontière ni entre individu et collectif ni entre moi et autrui, est remplacé par un moi qui prend la conscience de soi-même

121. Ibid., p. 8

122. GAO, X. J., « Ne pas avoir de -isme », dans Le Témoignage de la littérature, op. cit., p. 37. 123. Ibid., p. 37. 
et se distingue des autres. Les deux concepts du moi conduisent à deux libertés différentes, mais Gao change la "prémisse » et garde la " conclusion ». Il s'agit ainsi d'un paradoxe niché entre la tradition chinoise et le moderne occidental. Gao s'interroge:

Si un intellectuel chinois qui vit en Occident n'adopte pas de -isme et n'a pas recours uniquement à la tradition chinoise pour trouver un réconfort, peut-il encore protéger son indépendance d'esprit ${ }^{124}$ ?

En effet, la liberté de l'individu en Occident insiste plus sur l'effet collectif que sur la disponibilité taoïste qui est un état intérieur et qui ne dépend point d'autrui. C'est pourquoi Gao se demande encore: " La démocratie garantit-elle la liberté individuelle ${ }^{125}$ ? ":

La liberté de l'individu dans la vie moderne subit aussi de nombreuse restrictions touchant à ses conditions d'existence; en plus de pressions politiques et des contraintes sociales, il y a des limites sur le plan économique et éthique, et même des troubles d'ordre psychologique. Dès lors qu'un individu est vivant, il ne peut échapper totalement à ces problèmes existentiels ${ }^{126}$.

Même s'il existe en réalité pour l'individu une possibilité de liberté indépendante, Gao n'est pas sans avoir des doutes. Rappelons que ce que l'homme d'âge mûr dans La Fuite cherche est très exactement une liberté de soi-même qui ne dépende ni du mouvement social ni d'une idéologie et ni des autres. Mais en même temps, par la bouche de la jeune fille, Gao se demande si cette liberté qui renonce à tout n'offre pas qu'un moi désert.

La liberté totale de l'individu ne nous rend pas le paradis: voilà qui nous conduit à retourner à la question de l'origine culturelle du moi et de la subjectivité. Car chez Gao, la poursuite de la liberté de l'individu ne conduit pas au " connais toi toi-même » delphique. Même si la notion d'individu s'écarte chez lui de la catégorie traditionnelle et emprunte les concepts de sujet et de subjectivité à l'Occident, elle ne renvoie pas à l'Être de l'ontologie européenne, mais à un " existant » qui se passionne pour la vie elle-même...

124. Ibid.

125. GAO, X. J., De la création, Paris, Seuil, 2013, p. 296.

126. Ibid., p. 298. 



\title{
Autour des notions de temps et d'ombre de la subjectivité
}

\author{
Mise en regard de la modernité \\ chez Gao, Beckett et Lu Xun
}

Si une angoisse encore m'étreint, c'est de sentir cet impalpable instant glisser entre mes doigts comme les perles du mercure. Laissez donc ceux qui veulent se séparer du monde. Je ne me plains plus puisque je me regarde naître. Je suis heureux dans ce monde car mon royaume est de ce monde. Nuage qui passe et instant qui pâlit. Mort de moi-même à moi-même.

Albert Camus, Carnets I

\section{LES CONCEPTS DE SUJET ET DE TEMPS DANS L'ARRÊT D'AUTOBUS ET EN ATTENDANT GODOT}

\section{Un présent éternel et un présent absent}

La première pièce de théâtre de Gao, L'Arrêt d'autobus («车 站 ", de 1981, écrite à la même époque que le Premier essai sur la technique du roman moderne), a été considérée par des critiques chinois comme du théâtre de l'absurde, comme une version chinoise d'En attendant Godot ${ }^{1}$ dont l'auteur, Samuel Beckett, est considéré

\footnotetext{
1. On trouvera notamment ce genre de critiques de l'époque chez Chen Shouzu (陈 瘦竹) qui disait dans son article 《论荒诞戏剧的衰落及其在我国的影响 》 (《De la décadence du théâtre de l'absurde et de son influence dans notre pays [en Chine] », recueilli dans 《社会科学评论》 Shehui kexue pinglun, 1985, nº 11) que l'auteur de L'Arrêt d'autobus n'était en fait que l'apprenti d'En attendant Godot. On trouvera aussi les plus acerbes commentaires dans « «车站 》三人谈 » ( L'entretien entre trois personnes à propos de L'Arrêt d'autobus », dans 《戏剧报 》 Xijubao, 1984, n³ 3, dont les auteurs sont Tangyin 唐因, Du Gao 杜高 et Zheng Bo'nong 郑伯农), qui ne voient dans L'Arrêt qu'une imitation du théâtre de l'absurde.
} 
souvent comme, à la fois, l'un des derniers modernistes et l'un des premiers postmodernes. Certes, L'Arrêt d'autobus a bien été influencé par En attendant Godot: Gao reconnaît avoir lu cette pièce avant d'écrire L'Arrêt d'autobus; elle l'avait beaucoup touché2.

L'Arrêt d'autobus présente un groupe de passagers qui attendent l'autobus à un arrêt désaffecté - personne ne semble le savoir. En attendant l'autobus, les passagers papotent sur ce qu'ils veulent faire dans la ville. Soudain, un an s'est passé, les passagers sont perplexes et se demandent s'il faut continuer à attendre l'autobus. Puis dix ans s'envolent encore. Les passagers se trouvent toujours au même endroit: ils se plaignent de l'autobus qui ne viendra jamais, excepté un homme silencieux, d'âge mûr qui part à pied aussitôt. Finalement, ceux qui restent décident de partir ensemble à pied vers la ville pour ne plus perdre de temps.

Bien que le temps soit le référent clé dans les deux pièces, celle de Beckett et celle de Gao, la signification en est radicalement différente chez les deux auteurs. Dans En attendant Godot où l'allusion religieuse est très présente, le rapport du sujet au temps semble être à la fois opposé et proche de celui que définit saint Augustin, que Beckett a beaucoup lu. Dans Les Confessions, saint Augustin expose, sans doute pour la première fois dans l'histoire de la pensée, le propre du sujet-moi dans son rapport avec le temps: "Je mesure le mouvement des corps à l'aide du temps, et le temps luimême, ne puis-je donc le mesurer ${ }^{3}$ ? " Le temps est la mesure du sujet, et réciproquement: à partir de là, saint Augustin établit un rapport cohérent entre les deux. Ainsi il déclare: « je sais que si rien ne passait, il n’y aurait pas de temps passé; si rien n'arrivait, il n’y aurait pas de temps à venir; si rien n'était, il n'y aurait pas de temps présent ${ }^{4}$. " Puis il va encore plus loin: "Quant au présent, s’il était toujours présent, s'il n'allait pas rejoindre le passé, il ne serait pas du temps, il serait l'éternité ${ }^{5}$. Pour saint Augustin, le temps et le

2. GAO, X. J., La Poursuite d'un théâtre moderne, op. cit., p. 167.

3. Augustin (saint), Les Confessions, traduites par Joseph Trabuco, Paris, Flammarion, 1964, p. 275.

4. Ibid., p. 264

5. Ibid. 
sujet sont en telle cohérence que le temps n'est rien d'autre qu'une distension de l'âme $e^{6}$.

Beckett déploie sur la scène un "présent éternel ", dans la mesure où il n’y a pas de passé différencié: " rien n'était » dans l'attente de Vladimir et d'Estragon; ils ne savent pas qui est ce Godot qu'ils attendent, ils ne savent pas non plus pourquoi l'attendre. Le temps n'a pas disparu à cause de ce "non-sens »: il est là, mais ne s'incarne qu'au présent. Puisque hier est comme aujourd'hui, demain sera identique à aujourd'hui: le temps devient le véritable ennui pour les deux vagabonds. Si la pensée de saint Augustin implique, de façon religieuse, une cohérence entre sujet/conscience de soi et le temps, alors Vladimir et Estragon n'en sont effectivement pas sortis. Beckett ne voulait peut-être qu'aller jusqu'au bout de cette cohérence en abolissant le sens du temps, mais il évidencie finalement un autre rattachement indéfectible: le temps devient une prison éternelle pour le sujet. La critique que formule Beckett à propos de Proust est le meilleur commentaire de ses propres ouvrages, où la situation vécue est une impasse pour l'homme qui la vit: "Nous ne nous échappons ni des heures ni des jours. Pas davantage de demain que d'hier ${ }^{7}$. " C'est que la notion de temps est née de la conscience de l'Être et qu'elle y adhère impitoyablement.

C'est pourquoi le temps est comme un secret conventionnel, un "non dit " faisant presque tabou dans la pièce. Quand Vladimir touche ce point de secret du temps, par mégarde, Pozzo se met immédiatement en colère:

Pozzo (soudain furieux). - Vous n'avez pas fini de m'empoisonner avec vos histoires de temps? C'est insensé! Quand! Quand! Un jour, ça ne vous suffit pas, un jour pareil aux autres, il est devenu muet, un jour je suis devenu aveugle, un jour nous deviendrons sourds, un jour nous sommes nés, un jour nous mourrons, le même jour, le même instant, ça ne vous suffit pas ${ }^{8}$ ?

Dans L'Arrêt d'autobus, rien de tel: le temps n'est ni métaphore de la prison éternelle, ni objet indissociable du sujet. Le rapport

6. Ibid., p. 275.

7. BECKETT, S., Proust, traduit de l'anglais par Edith Fournier, Paris, Minuit, 1990, p. 24.

8. BECKett, S., En attendant Godot, Paris, Minuit, 1952, p. 126. 
entre lui et le sujet reste équivoque. Cet histoire de passagers attendant en vain un autobus qui ne viendra jamais pointe l'expérience collective éprouvée pendant les dix ans de la révolution culturelle plutôt qu'elle n'est la métaphore du temps perdu. Il y a une sensation d'absence du présent: il semble que la ville soit le signe du futur et que l'autobus absent qui aurait dû prendre en charge les passagers est ce présent qui n'existe pas. En d'autres termes, les passagers se trouvent abandonnés dans un "non-temps".

Il y a donc deux sortes différentes de métamorphose du temps respectivement chez Beckett et chez Gao. Dans En attendant Godot, le temps reste stagnant et devient une prison pour le sujet; dans L'Arrêt d'autobus, les passagers se situent dans une faille du temps sans s'en rendre compte et sans la percevoir. Il y a une tendance similaire à la perte du futur, au renoncement forcé et plus au moins comateux à ce dernier: chez Beckett, le présent devient quelque chose d'inextricable qui demeure perpétuellement; chez Gao, le présent échappe aux passagers. Il est donc indiscutable que la sensation de l'absurde est au rendez-vous dans les deux pièces, mais il n'est pas moins évident que l'absurde, dans les deux cas, se fonde sur des conventions partagées entre l'auteur et les spectateurs qui ne sont pas du tout les mêmes, et qui relèvent de psychologies sociales fort distinctes.

Ainsi, les questions se posent différemment ici et là: comment le temps peut-il exister en dehors du sujet, ou, réciproquement, le sujet peut-il exister en dehors du temps dans L'Arrêt d'autobus? Pour quelle raison le sujet n'arrive-t-il pas à se détacher du temps dans En attendant Godot?

\section{Le rapport entre le sujet et le temps}

Gao a fortement insisté sur le fait que L'Arrêt d'autobus n'est en rien une imitation d'En attendant Godot; comme il le souligne, «les personnages d'En attendant Godot sont des signes abstraits, alors que dans L'Arrêt d'autobus, ils sont au contraire des personnages concrets $^{9}$ ». À la vérité, la clef de la différence et dans le mode d'inscription du désir dans le rapport au temps.

9. GAO, X. J., La Poursuite d'un théâtre moderne, op. cit., p. 127. 
La critique que Beckett fait de Proust peut être considérée, on l'a dit, comme une bonne interprétation de ses propres ouvrages, notamment En attendant Godot. Quand Beckett lit $\grave{A}$ la recherche du temps perdu, il trouve que Proust condamne ses personnages à être les victimes de la " circonstance prépondérante qu'est le temps ":

[...] des victimes comme certains organismes inférieurs qui n'ont conscience que de deux dimensions et qui, se trouvant soudain confrontés au mystère de la hauteur, en deviennent les victimes - les victimes et les prisonniers. Nous ne nous échappons ni des heures ni des jours. Pas davantage de demain que d'hier. Nous ne nous échappons pas d'hier car hier nous a déformés, à moins que nous ne l'ayons déformé. Peu importe la couleur du moment, il y a eu déformation. Hier n'est pas un jalon que nous aurions dépassé, c'est un caillou des vieux sentiers rebattus des années qui fait partie de nous irrémédiablement, que nous portons en nous; lourd et menaçant. Ce n'est pas seulement qu'hier nous a un peu plus épuisés; nous sommes différents, nous ne sommes plus ce que nous étions avant la calamité d'hier ${ }^{10}$.

Ce que Beckett appelle " accomplissement ", c'est le moment où le sujet s'identifie à l'objet de son désir ${ }^{11}$. Mais la fissure qui, selon Beckett, est perçue par Proust sur l'apparence de l'accomplissement, est générée inévitablement par le temps: "Nos désirs d'hier, qui valent pour notre moi d'hier, ne valent plus pour notre moi d'aujourd'hui ${ }^{12}$. " Le désir devance toujours le temps, tandis que le sujet désirant est déjà mort dans la poursuite de son désir — « sans doute plusieurs fois en route ${ }^{13}$ ". Estragon et Vladimir sont sans doute deux exemples de "sujet mort »: ils font semblant d'être des sujets désirants mais ne savent même pas ce qu'est Godot. Pour ainsi dire, ce qu'ils attendent n'est même pas un objet désiré; Godot n'existe qu'en syllabes phonétiques. Dès lors, l'« attente " perd son sens propre - atteindre un but - et se réduit à une convention tacite: elle est là, elle impose sa figure à la fois quotidienne et arbitraire à laquelle il n'est pas possible d'échapper et qui conduit au bourbier

10. BeCKetT, S., Proust, op. cit., p. 23-24.

11. Ibid., p. 24.

12. Ibid., p. 23-24.

13. Ibid., p. 24. 
du néant: « Que l'objet soit bien ou mal disposé, voilà qui n’a aucune réalité, aucune signification ${ }^{14}$."

Adorno considère que Beckett procède à une liquidation et à une exécution du sujet ${ }^{15}$. De fait, Beckett tente de renoncer non seulement au sens de l'objet désiré, mais aussi au sens du sujet lui-même, en mettant en évidence une fissure entre le sujet et le temps. Mais cette fissure ne peut apparaître que parce qu'il y a du " fissurable ", parce qu'existe culturellement un préalable de la cohérence temps/ sujet.

Si En attendant Godot révèle un tel double non-sens, du sujet et de l'objet, dans L'Arrêt d'autobus, les désirs des passagers sont en revanche très concrets et - dans un monde normal - faciles à combler. Pour le vieil homme, une partie d'échecs; pour le voyou, le yaourt de dernière marque; pour la jeune fille, un rendez-vous amoureux, pour le jeune garçon portant lunettes, le cours d'anglais; pour la mère, rejoindre sa famille; pour le menuisier, un travail à la ville et pour le directeur Ma, un repas auquel il est invité. Bref, toutes ces « bagatelles " n'ont rien à voir avec un profond thème philosophique universel, ni avec le tragique de la condition humaine. D’ailleurs, les dix ans d'attente n'abîment pas leurs désirs futiles; les personnages restent motivés et optimistes - y compris le voyou et le bureaucrate typique, le directeur Ma. L'attente des passagers ne provoque pas ce sentiment d'anxiété qui peut conduire au néant, et le temps perdu ne fait pas surgir de grande réflexion sur le rapport entre le sujet et le temps.

Concluons: même si l'Arrêt d'autobus emprunte effectivement la forme et le thème de l'" attente " à En attendant Godot, et exprime aussi des doutes sur son époque, cette pièce de théâtre ne partage ni le même rapport entre le sujet désirant et l'objet désiré, ni la difficulté beckettienne: Gao ne s'inscrit pas dans la même école de l'absurde. Hors la scène, les deux dramaturges n'ont pas les mêmes perspectives sur le rapport sujet/temps; perspectives qu'ils partagent chacun avec sa culture originelle. C'est pourquoi, à l'opposé

14. Ibid., p. 23.

15. Adorno, T. W., Note To Literature, Columbia, Columbia University Press, Reprint édition, 1991. 
d'En attendant Godot où le sujet ne fait que se profiler sur le temps, dans L'Arrêt d'autobus, le sujet existe en dépit de l'absence de présent: la "logique " est ici celle d'un rapport incohérent entre le temps et le sujet.

Même si En attendant Godot opère une double renonciation, à l'éternité théologique et à l'ontologie - sur laquelle s'est fondé le concept du temps occidental - la notion du temps ne sort finalement pas chez Beckett du champ relationnel subjectivisme/ objectivisme. Ce temps naît, comme le veut la tradition, dans la relation du sujet et de l'objet, il ne surgit pas de "mon rapport avec les choses ", pour emprunter l'expression de Merleau-Ponty ${ }^{16}$. Dans Godot, le sujet qui se projette sur le temps se trouve dans la même situation que Tantale, qui subit en permanence une rupture d'équilibre « à cette différence près que nous cédons, nous, à la tentation, et que le motif perpétuel de nos désillusions présente peut-être une plus grande variété17 $"$.

La notion occidentale de temps n'existe pas dans la pensée chinoise avant le XIX ${ }^{e}$ siècle. Les Chinois l'ont adoptée il n'y a qu'une centaine d'années. Dans la tradition, le temps est compté sur la base du cycle sexagésimal (天干地支) et du cercle des « saisons »; il n’y a ni de début, ni de terme: il n'est besoin ni d'un point de départ pour marquer l'origine de l'homme, ni d'un terme pour exercer le jugement final. Il y a là une bifurcation première et essentielle, voire la source évidente de l'« écart » de compréhension entre pensée occidentale et pensée chinoise:

Partie d'une conception cosmogonique, la Grèce a dépassé celle-ci, à l'âge de la philosophie, par l'onto-théologie: en pensant l'Être ou Dieu; de là, le statut fondateur qu'elle accorde à l'éternel, celui-ci étant le socle sur lequel elle a stabilisé le cosmos qu'elle a tiré du chaos. Tandis que, déployant une conception cosmologique qui n'a jamais été hantée par le chaos, l'« origine » n'étant pour elle qu'une indifférenciation primitive, la Chine en approfondit la pensée en celle du procès du monde et de sa régulation, qu'elle appelle le tao ou la « Voie ». De là, l'attention qu'elle porte au « constant » comme principe d'une harmonie continue révélant la cohérence propre à tout

16. Merleau-PontY, M., Phénoménologie de la perception, Gallimard, 1945, p. xc471.

17. BECKETT, S., Proust, op. cit., p. 24. 
réel, tout réel étant en procès; en même temps que le caractère non théorique de sa pensée du pérenne ${ }^{18}$.

De même, la notion de sujet n'a jamais fait l'objet de discussions dans la pensée chinoise traditionnelle. Sans le récif constitué par le sujet, il y n'aurait tout simplement pas, dans le long courant du temps, d'émergence d'un quelconque " présent » à l’opposé du passé ou du futur.

Aussi, bien que le sujet/moi chinois soit pris dans le courant de la " modernisation " et fasse plus ou moins sienne la notion de temps importée d'Occident (à l'instar de Dieu et de l'Être), les choses ne sont pas égales: la spéculation sur le «temps " dans la pensée chinoise moderne et dans la littérature se démarque de ce qu'elle est en Occident. En d'autres termes, le concept de temps en Chine, même modernisé et occidentalisé, garde toujours ses distances ${ }^{19}$; il y a écart.

Dans En attendant Godot, le renoncement à l'immortalité promise par la religion et la dérision envers la notion de temps impliquent de détrôner Dieu, en qui s’incarnait jadis la vérité absolue. Ainsi le temps ne devient-il qu'un concept abstrait — il révèle le destin comparable et lié du moi-sujet: c'est de là que naît l'absurdité. Dans

18. JULLIEN, F., Du «temps », éléments d'une philosophie du vivre, Paris, Grasset \& Fasquelle, 2001, p. 20-21. En ce qui concerne la notion de temps dans la philosophie contemporaine occidentale, l'auteur révèle donc la relation indissociable avec la notion d'éternité. «Certes, la philosophie n'a cessé de dire son dédain, au Xxe siècle, pour cette notion, renvoyée au registre de la foi, et à l'approche ontologique du temps qui en a découlé (chez Bergson aussi bien que chez Heidegger) : l'éternité ne serait que le concept vide d'un être permanent qui, loin d'être à l'origine du temps, est en fait dérivé de notre expérience ordinaire de la temporalité. Reste qu'on ne peut oublier que c'est aussi par elle, l'éternité, que nous avons édifié la notion de temps; dès lors, et quelles que soient les critiques ultérieures à son égard, notre concept du temps en porte la trace indélébile, ne serait-ce que dans sa propre possibilité : l'héritage est si bien assimilé que nous ne le percevons plus, mais nous en dépendons toujours. »

19. On peut interpréter cela comme un phénomène général lorsqu'il s'agit d'adopter la catégorie occidentale au cours du processus de modernisation. Voir Du «temps ", éléments d'une philosophie du vivre, op. cit., p. 36: "Les penseurs chinois d'aujourd'hui ont euxmêmes été gagnés, au contact de l'Occident, à cette idée d'un concept de droit du temps. Car, d'une façon générale, le concept une fois rencontré, sa commodité éprouvée, on ne saurait désormais s'en passer, et cela au prix des pires distorsions, comme toute l'histoire de la pensée chinoise et japonaise du $x x^{e}$ siècle le prouve assez; le concept n'était peutêtre pas universel dans sa compréhension, mais il le paraît désormais de par l'uniformité de son usage. » 
L'Arrêt d'autobus, le sentiment de l'absurdité est plutôt provoqué par l'expérience commune, à la fois rocambolesque et réelle, vécue par tout un chacun à l'époque de la révolution culturelle. En dépit de l'influence des pièces de théâtre occidentales contemporaines (celles de Brecht, d'Antonin Artaud et bien sûr de Beckett), L'Arrêt d'autobus montre que le temps est très précieux et cher pour les passagers. Il n’y a ni perplexité à l'égard de la théologie, ni nécessité de s'appuyer sur l'ontologie: le temps est là comme quelque chose qui a sa propre réalité, sans lien avec la notion de vie ou de mort. Lorsque les passagers perçoivent qu'un an s'est envolé d'un coup, ils ne cherchent ni la cause de la perte de temps, ni la raison pour laquelle ils attendent en vain l'autobus: au contraire, ils parlent de leurs "capacités " et de leurs mérites (le talent aux échecs ou le savoir-faire en tant qu'artisan), et de leurs besoins ou désirs (participer aux examens, se rendre à un rendez-vous amoureux) afin de prouver leur « légitimité » à prendre l'autobus pour aller à la ville. Le temps n'est pas un concept abstrait; il est celui de la vie quotidienne où se distinguent des moments liés aux circonstances de la vie.

\section{La montre et les temps modernes}

Il y a deux notions de temps entremêlées dans L'Arrêt d'autobus. Elles sont représentées respectivement par le groupe de passagers et par l'homme silencieux: un homme d'âge mûr qui ne se joint jamais aux conversations des autres passagers et qui n'attend pas en vain le bus absent, puisqu'il part seul aussitôt. Cet homme silencieux, beaucoup de critiques supposent que c'est la figure de l'auteur lui-même. Mais pour Gao, il est plutôt la représentation d'un autre moi chez les passagers:

Il existe dans l'esprit de chaque passager comme une voix intérieure. Lorsque la jeune fille dit que cet homme silencieux lui jette un coup d'œil, c'est en effet qu'elle perçoit qu'il la voit - car ce qu'elle voit n'est qu'elle-même ${ }^{20}$.

En tant que figure du subconscient individuel, l'homme d'âge mûr compose alors le scénario latent qui contraste avec le scénario patent où les passagers représentent une conscience collective:

20. GAO, X. J., La Poursuite d'un théâtre moderne, op. cit., p. 187. 
deux notions de temps s'opposent. L'une, celle des passagers, présente le point de vue du mécanisme historique: le progrès permanent conduira à un but supérieur (par exemple, le communisme est l'étape supérieure de la société humaine). Mais cette perspective s'entremêle en même temps à une tradition psychosociale qui s'incarne dans "le moment-occasion " (时机). Une occasion qui se manifestera d'elle-même dans le cours du temps sans que le sujet la cherche par lui-même. Cela explique pourquoi, lorsque les passagers réalisent qu'un an s'est envolé, ils ne réagissent pas, ne veulent ni retourner chez eux, ni chercher une autre moyen pour aller à la ville.

L'autre point de vue sur le temps est celui de l'homme silencieux; ce point de vue est porteur d'un doute sur la notion de temps se dirigeant vers un grand but et sur celle de progrès continu. Ainsi, lorsque cet homme part tout seul, il montre bien quelle est son attitude - et aussi celle de l'auteur — vis-à-vis du temps " moderne »: "La société continue toujours à progresser: cette idée depuis un siècle paraît bien douteuse ${ }^{21}$. »

Il convient de mettre en parallèle les deux scènes qui suivent d'En attendant Godot et de L'Arrêt d'autobus pour comparer la façon dont les personnages révèlent leur notion du temps.

\section{En attendant Godot:}

Pozzo. - Mais c'est tout naturel, c'est tout naturel. Moi-même, à votre place, si j'avais rendez-vous avec un Godin... Godet... Godot... enfin vous voyez qui je veux dire, j'attendrais qu'il fasse nuit noire avant d'abandonner. (Il regarde le pliant.) J'aimerais bien me rasseoir, mais je ne sais pas trop comment m'y prendre.

Estragon. - Puis-je vous aider?

Pozzo. - Si vous me demandiez, peut-être?

Estragon. - Quoi?

Pozzo. - Si vous me demandiez de me rasseoir.

[...]

Pozzo. - Vous avez sans doute raison. (Il se rassied.) Merci, mon cher. Me voilà réinstallé. (Estragon se rassied. Pozzo regarde sa montre.) Mais il est temps que je vous quitte, si je ne veux pas me mettre en retard.

Estragon. - Le temps s'est arrêté.

21. GAO, X. J., «没有主义» (Ne pas avoir de-isme), op. cit., p. 13. 
Pozzo (mettant sa montre contre son oreille). - Ne croyez pas ça, monsieur, ne croyez pas ça. (Il remet la montre dans sa poche.) Tout ce que vous voulez, mais pas ça.

[...]

Pozzo. - [...] Merci, messieurs, et laissez-moi vous - (il fouille dans ses poches) - vous souhaiter - (il fouille) - vous souhaiter - (il fouille) mais où ai-je donc mis ma montre? (Il fouille.) Ça alors! (Il lève une tête défaite.) Une véritable savonnette, messieurs, à secondes trotteuses. C'est mon pépé qui me l'a donnée. (Il fouille.) Elle est peutêtre tombée. (Il cherche par terre, ainsi que Vladimir et Estragon. Pozzo retourne de son pied les restes du chapeau de Lucky.) Ça, par exemple!

Vladimir. - Elle est peut-être dans votre gousset.

Pozzo. - Attendez. (Il se plie en deux, approche sa tête de son ventre, écoute.) Je n'entends rien! (Il leur fait signe de s'approcher.) Venez voir. (Estragon et Vladimir vont vers lui, se penchent sur son ventre. Silence.) Il me semble qu'on devrait entendre le tic-tac.

Vladimir. - Silence!

(Tous écoutent, penchés.)

Estragon. - J'entends quelque chose.

Pozzo. - Où?

Vladimir. - C'est le cœur.

Pozzo (déçu). - Merde alors ${ }^{22}$ !

Dans L'Arrêt d'autobus:

Le menuisier. - Excusez-moi, vous avez l'heure à votre montre?

Celui qui porte des lunettes (regarde sa montre, étonné). - Comment?

Comment...

Le menuisier. - Elle ne marche plus?

Celui qui porte des lunettes. - C'est mieux si elle ne marche plus... Comment c'est possible, un an s'est passé!

La fille. - Tu mens!

Celui qui porte des lunettes (regarde encore sa montre). - C'est vrai, Nous avons déjà attendu à cet arrêt une année pleine!

(Le loubard met son index dans la bouche, et siffle un coup)

Le vieil homme (Il leur jette un regard appuyé). - Vous déconnez.

Celui qui porte des lunettes. - Je ne déconne pas, regardez votre montre.

Le menuisier. - Ne sifflez pas, mais ce n'est pas possible.

La mère. - Pourquoi ma montre marque qu'il est seulement deux heures quarante?

22. BECKETT, S., En attendant Godot, op. cit., p. 49-64. 
Le loubard. - (se rapproche). Elle s’arrête!

Le menuisier. - Ne criez pas, (se tourne vers le vieil homme). Regardez votre montre.

Le vieil homme. - (En tremblant, il sort enfin sa montre de sa poche.)

Ce n'est pas correct?

Le loubard. - Vous la regardez à l'envers.

Le vieil homme. - Une heure... dix. Ma montre s’arrête.

[...]

Le directeur Ma. - (Agite son poignet et l'écoute). Ma montre s'arrête aussi.

La mère. - Regardez la date, n'est-ce pas votre montre avec le calendrier?

Le directeur Ma. - Le $48^{\mathrm{e}}$ jour dans le $13^{\mathrm{e}}$ mois - c'est bizarre, ma montre est de marque Omega importée!

[...]

Celui qui porte des lunettes. - La mienne est une montre électronique, elle ne se trompe pas. Regardez, elle marche toujours. Je l'ai achetée l'année dernière, elle ne s'arrête jamais. C'est une montre multifonctions, qui indique bien l'année, le mois, le jour, l'heure, la minute et la seconde. Regardez, c'est sûr qu'une année pleine s’est passée!

Le menuisier. - Tu paniques tout le monde. Même si ta montre est électronique, et alors? Il est possible que ta montre électronique ne marche pas bien.

Le vieil homme. - Mais nous ne pouvons pas, ne pas croire la science. L'électronique est la science, la science ne nous ment pas. Maintenant c'est l'époque électronique! Certainement, c'est quelque chose en nous qui ne va pas.

La mère. - Ça veut dire que, nous avons attendu l'autobus à cet arrêt pendant une année pleine?

Celui qui porte des lunettes. - Oui, vraiment une année. Un an et trois minutes une seconde, deux secondes, trois secondes, quatre secondes, cinq secondes, six secondes... Regardez, elle marche toujours $^{23}$.

Pozzo est tourmenté par le temps, même s'il veut s'en détourner: il ne peut rester à attendre Godot comme Estragon et Vladimir, mais il ne sait pas non plus pourquoi il doit partir. La montre de Pozzo est un signe particulier: elle représente un concept du temps au sens traditionnel (la montre de Pozzo est héritée de son grandpère) - autrement dit, un temps qui adhère à cette raison à laquelle

23. GAO, X. J., «车站 》 (L'Arrêt d'autobus), Taibei, Lianhe Wenxue, 2001, p. 44-47. Nous traduisons. 
les personnages renoncent en déclarant leur ennui. Aussi la montre révèle-t-elle le doute de Pozzo sur le temps: elle n'est mentionnée que deux fois dans la pièce, négativement. La première fois, Pozzo sort sa montre et l'écoute, puis déclare sa méfiance ( « on peut croire tout ce qu'on veut, mais pas ça »). La deuxième fois, Pozzo perd sa montre, il la cherche partout et croit qu'elle est dans son ventre (l'action signifie sans doute qu'il cherche le temps/la raison en lui-même). Puis il se rend compte que le tic-tac n'est que la pulsation de son cœur. Beckett montre de manière allégorique que Pozzo se méfie du temps mais qu'il ne peut pas non plus s'en détacher: la montre intériorisée devient une partie de son corps, mais aussi de son âme - le cœur.

La montre a également plusieurs significations pour les passagers de L'Arrêt d'autobus: il y a d'abord les temps variés marqués par chacune des montres des passagers (deux heures quarante, une heure dix, le $48^{\mathrm{e}}$ jour dans le $13^{\mathrm{e}}$ mois). Par ailleurs, les montres un modèle ancien quelconque pour le vieil homme ou la nouvelle montre luxueuse du directeur $\mathrm{Ma}$ - se sont arrêtées pendant l'attente de l'autobus, à l'exception de la montre électronique du jeune portant lunettes. Autrement dit, seule la montre électronique révèle quelque chose d'incontestablement " correct ". C'est une scène comique plutôt qu'absurde ou tragique. Voici le "syllogisme " que le vieil homme démontre: l'électronique est la science, la montre électronique est la science, la science ne nous ment pas, donc la montre électronique ne nous ment pas; de plus, nous sommes à l'époque de l'électronique, la science ne nous ment pas, l'époque de l'électronique ne saurait avoir tort, donc, ce sera "quelque chose en nous qui ne va pas ".

Si le trajet entre l'arrêt et la ville est aussi la métaphore du processus de modernisation, l'autobus qui ne vient jamais n'implique pas seulement un présent absent, mais aussi l'absence du vecteur culturel pour aller à «l'autre rive ", si bien que les passagers restent toujours au point de départ en imaginant leurs vies dans la ville: tout cela révèle que la difficulté de la modernisation vient aussi du sujet lui-même, qui n'a pas encore pris conscience d'être un individu indépendant. De même, si la montre est un signe de modernité, la variation des temps indiqués par les montres révèle le disparate des attitudes adoptées par les passagers face au temps moderne. La montre électronique, symbole de la science et mesure la plus 
rassurante pour savoir quel est le "vrai » temps, démontre pourtant une réalité absurde et justifie la désillusion où s'engloutissent les rêves et les désirs des passagers. Ce doute ne porte pas seulement sur les dix ans de la révolution culturelle; il atteint le temps moderne en lui-même et la soumission à celui-ci - pour le dire plus précisément, le temps linéaire pourvu d'un aboutissement.

Il y a deux rapports opposés entre le sujet et le temps. Celui du " rattachement " chez Beckett: le temps est le destin impossible à éviter pour le sujet, il est l'ombre étouffante aussi bien que la prison éternelle. Et celui de l'" incohérence " chez Gao: le temps est presque quelque chose d' "indifférent " pour les passagers, bien qu'ils veuillent le rattraper. Autrement dit, il y a toujours tendance à ce que le temps passe à côté de l'homme, ou que l'homme passe à côté du temps. C'est pourquoi, à l'opposé d'En attendant Godot où le sujet se projette sur le temps, L'Arrêt d'autobus montre une sorte de parallélisme du sujet et du temps. En outre, si la notion de temps en Occident s'appuie sur la spéculation conceptuelle plus que sur la vie quotidienne, en Chine, elle s'oriente plutôt vers les notions de morale et de valeur dans leurs implications quotidiennes. Chez Beckett, de façon récurrente, la rébellion contre le concept du temps ne provoque pas seulement un combat contre l'idée d'éternité, d'immortalité, elle pousse le conflit contre l'être humain lui-même. Les questions sans réponse qui se répètent dans ses ouvrages deviennent la punition de Sisyphe: « où maintenant? quand maintenant? qui maintenant ${ }^{24}$ ? "... Dans les œuvres de Gao, au contraire, il y a une répétition de l'histoire qui réinstalle le temps dans " un cercle ". D’un an en dix ans, les passagers répétant leur attente, le temps linéaire accomplit aussi sa métamorphose en un temps circulaire. Gao raconte une autre histoire dans La Montagne de l'âme: les habitants de deux villages relancent de génération en génération la même vendetta. Mais dans ces batailles qui ne finiront jamais et qui ne répètent qu'un seul acte - celui de la vengeance -, les personnages sont effacés en tant qu'individus: il n'y a que le temps qui tourne sur lui-même dans un même espace (les deux villages) et, se perpétuant, une même psychologie sociale éternelle:

24. BeCKetT, S., L'Innommable, Paris, Minuit, 1953, p. 7. 
Les femmes sortent des maisons en essuyant leurs larmes et guettent l'entrée du village, regardant les hommes frapper les gongs, couteau à la main et fusil à l'épaule. Ils poussent de grands cris en descendant de la montagne, pour les ancêtres, pour le clan, pour la terre et les forêts, pour leurs descendants, ils s'entre-tuent à coups de fusil, puis, discrètement, ils rapportent les cadavres. Ensuite, les femmes se remettent à crier pour invoquer ciel et terre. Puis le calme revient. Se succèdent les labours, les semailles, le repiquage, les moissons et le battage des grains. Le printemps passe et l'automne arrive, les hivers succèdent aux hivers, quand les tombes sont envahies par les herbes, les veuves ont volé les jeunes gens, les orphelins ont grandi et sont devenus adultes, la tragédie a été oubliée, seule la gloire des ancêtres reste dans les mémoires. Jusqu’à ce que, le soir du réveillon de Nouvel An, avant les sacrifices aux ancêtres, les vieux se mettent à raconter les querelles de famille d'autrefois, que les jeunes se mettent à boire, que leur sang chaud montre à nouveau dans leurs veines ${ }^{25} . .$.

\section{DE Lu Xun À GaO XingJian: LA BIFURCATION DE LA SUBJECTIVITÉ}

\section{L'Arrêt d'autobus et Le Passant}

On peut, singulièrement, noter deux opinions opposées au sujet de L'Arrêt d'autobus: les critiques chinois le considèrent comme un texte imité de Beckett et rédigé sous l'influence de la philosophie existentialiste, tandis que les critiques sinologues d'outre-mer croient que cette pièce de théâtre de Gao a un lien avec Le Passant de Lu Xun ${ }^{26}$. Or, ces deux pièces, En attendant Godot et Le Passant, s'opposent pratiquement l'une à l'autre: la pièce de Beckett, qui se moque de la raison et de la logique en faisant désespérer du

25. GAO, X. J., La Montagne de l'âme (traduit du chinois par Noël et Liliane Dutrait), Paris, l'Aube, 1995, p. 303.

26. Par exemple, Quah Sy Ren remarque que l'homme silencieux de L'Arrêt d'autobus incarne la figure du passant chez Lu Xun. D'après Quah, Gao emprunte ce personnage luxunien pour se faire plus aisément adopter par les spectateurs chinois, et aussi pour détourner l'attention des censeurs : voir Gao Xingjian and Transcultural Chinese Theater, Hawai'i, University of Hawai'i Press, 2004. Sébastian Veg, comparant aussi les deux pièces de théâtre, montre que les deux auteurs insistent sur la nécessité de se frayer un chemin personnel à travers les buissons de la vie : voir « De la marginalité à l'individualité dans les premières pièces de Gao Xingjian », dans L'écriture romanesque et théâtrale de Gao Xingjian, sous la direction de Noël Dutrait, Paris, Seuil, 2006, p. 147-167. 
destin de l'être humain, relève typiquement de l'« anti-héroïsme »; en revanche, dans la pièce de Lu Xun, le passant, personnage pour ainsi dire nietzschéen, est une figure de tragédie héroïsée. Comment peut-on dès lors concevoir que L'Arrêt d'autobus entre en résonance à la fois avec En attendant Godot et avec Le Passant?

Gao connaît bien Le Passant, la seule pièce de théâtre de Lu Xun. Dès son Premier essai sur la technique du roman moderne et maintes fois par la suite, il souligne la modernité des ouvrages de Lu Xun, notamment celle du Journal d'un fou et du pPassant:

Le Journal d'un fou a emprunté l'absurde à Gogol qui était bien antérieur aux ouvrages de Kafka; tandis que dans Le Passant, son style n'est pas loin de celui de Pirandello: à cette époque, les théâtres d'Ionesco et de Beckett n'ont pas encore paru ${ }^{27}$.

Le Passant est une pièce allégorique en un acte et trois personnages: le passant, le vieil homme (le grand père) et la fillette. Le portrait du passant ressemble à l'auteur lui-même: " entre trente et quarante ans, l'air fourbu et obstiné, le regard sombre et secret; moustache noire, cheveux hirsutes ${ }^{28}$ ". Il vient de l'est où il y a quelques taillis et des ruines, il se dirige vers l'ouest où il n'y a que des tombes abandonnées. Malgré la faim, la soif, la fatigue et la blessure de son pied, le passant continue à marcher en ignorant qui il est. Il ignore même d'où il vient et où il ira lorsque le vieil homme le lui demande:

Le fait est que je ne le sais pas. Du plus loin que je me souvienne, j'ai toujours marché ainsi, en route pour une destination qui gît quelque part en avant. Tout ce dont je me rappelle, c'est que j'ai fait un long chemin et que maintenant, me voilà ici. Je poursuivrai ma route par là (montrant l'ouest), en avant ${ }^{29}$ !

Marchant sans but déterminé, le passant est pourtant pressé par une voix mystérieuse, antérieure à lui-même, que le vieil homme entendait aussi quand il était jeune. Puis le passant se renseigne sur l'endroit vers où il se dirige, le vieil homme lui répond: « c'est des

27. GAO, X. J., Premier essai sur la technique du roman moderne, op. cit., p. 113.

28. LU, X., «过客》 (Le Passant); nous traduisons, sur la base de la traduction de Pierre Ryckmans, La mauvaise Herbe, Bibliothèque asiatique, Paris, 1975, p. 80-87.

29. Ibid. 
tombes », alors que la fillette réplique: "Non, non, il y a tout plein de lys sauvages et d'églantines, j'y vais jouer souvent, et je les regarde. » Il semble que les trois personnages représentent trois perspectives temporelles: vers le passé, le présent et le futur. L’ouest signifie, pour le vieil homme, la fin de la vie et la mort, mais il indique la joie et la vitalité pour la fillette; tandis que pour le passant, l'est et l'ouest révèlent respectivement l'Orient qu'il fuit et l'Occident où il ira - il éprouve pourtant perplexité et angoisse et le montre en prenant la direction de l'ouest.

Il y a dans L'Arrêt d'autobus aussi bien que dans Le Passant quelque chose d'impossible à tenir: le trajet du temps chez Gao et le trajet du voyage chez Lu Xun. Les passagers attendent le futur, mais le présent est absent; le passant marche sans cesse mais ne sait quel chemin il faut prendre. Ce trajet impossible à tenir semble une métaphore de la route de la modernisation en Chine, route sur laquelle les deux auteurs se posent bien des questions.

Le passant comme l'homme silencieux symbolisent sans doute le réveil de la conscience de soi et de la volonté individuelle qui survient au cours du trajet: tous deux sortent de la foule et choisissent de partir seuls. Chez Lu Xun le passant, qui a quelques traits de Zarathoustra, paraît un misanthrope:

Si je rebrousse chemin, je ne trouverai aucun endroit où il n’y aurait partout le règne des titres et les propriétaires, les expulsions et les geôles, partout s'affichent les sourires artificiels et les fausses larmes. Je les hais. Je ne retournerai pas en arrière ${ }^{30}$ !

Quant à l'homme chez Gao, il reste muet et part en silence: il est la figure de la conscience individuelle réprimée en lui-même par chaque passager. Il hésite aussi à faire son choix: il est nerveux comme les autres en attendant l'autobus, et lorsqu'il se met en route, " une légère musique sonne qui révèle sa poursuite douloureuse mais persistante ${ }^{31} "$.

Foucault caractérise comme suit la modernité en interprétant l'enthousiasme baudelairien devant les temps nouveaux: "rupture

30. Ibid.

31. GAO, X. J., L'Arrêt d'autobus, op. cit., p. 42. 
de la tradition, sentiment de la nouveauté, vertige de ce qui passe ${ }^{32}$ ". Chez Gao et chez Lu Xun, il y a aussi le sentiment du " vertige ", mais il s'agit plutôt d'un vertige du dépaysement ressenti devant la réalité. La modernité, pour les deux auteurs chinois, est d'emblée un indicateur de la valeur de l'individu, capable de s'opposer à la psychologie sociale de leurs époques, et surtout de se placer en confrontation avec l'éthique traditionnelle. Tant il est vrai que la subjectivité individuelle ne se forme pas dans les discussions philosophiques et spéculatives, mais dans la lutte avec la réalité, c'est-à-dire avec la politique, la société et aussi la morale traditionnelle.

De ce point de vue, l'" absurde " qui apparaît chez les deux auteurs est à la fois une façon de protester contre la réalité et une manière de donner sens à l'existence. Ainsi, l'humanisme - qui est pour Gao l'un des caractères majeurs du modernisme chinois, à l'époque du 4 mai comme dans les années 1980 - permet de distinguer L'Arrêt d'autobus d'En attendant Godot. Le même humanisme fait aussi que Gao se distingue lui-même des modernistes occidentaux et rejoint plutôt les premiers modernistes chinois du début du $\mathrm{xx}^{\mathrm{e}}$ siècle. Ce qu'il importe ici de souligner, c'est que l'humanisme chez Gao (comme avant lui chez Lu Xun) ne se fonde pas sur une quelconque valeur universelle, ou pour mieux dire, portée par l'universalisme occidental (par exemple, l'égalité), mais sur les valeurs traditionnelles: l'humanisme de Lu Xun dérive de l'humanité (仁) confucéenne et celui de Gao découle de la disponibilité (自在) taoïste.

D'où le regard sentimental qu'on note dans les premières pièces de Gao. Les portraits des passagers de L'Arrêt d'autobus ne sont pas négligés comme un décor de scène: ils contrastent avec l'homme silencieux; ils sont des projections en miniature de la société; ils ne symbolisent pas forcément les " mauvais côtés » de la société comme maints personnages dans les œuvres du réalisme socialiste. Il y a de la compassion pour la jeune fille qui manque son rendez-vous amoureux, et aussi de l'ironie envers le directeur Ma qui prétend ignorer la queue et exhibe son «privilège officiel » quand un bus passe: " Lao Wang, chauffeur Wang! Je suis le directeur Ma

32. FoucAult, M., Dits et écrits II, Paris, Gallimard (Quarto), 2001, p. 1388. 
du magasin d'État ${ }^{33}$ ! " La pièce se termine même sur un " happy end »: le voyou devient l'apprenti du menuisier, la jeune fille trouve un autre prétendant (le garçon portant lunettes), et les passagers partent ensemble.

Pour cette raison sans doute, Sébastian Veg caractérise les deux premières pièces de théâtre de Gao, L'Arrêt d'autobus et Signal d'alarme, " comme des textes politiques, tributaires - fût-ce de façon critique - de l'esthétique réaliste socialiste qui faisait office de canon littéraire dans la Chine maoïste et dans les premières années postmaoïstes ${ }^{34}$ ". Il a sans doute raison, notamment pour la pièce Signal, écrite juste après L'Arrêt sur la proposition de Yu Shizhi, le directeur du Théâtre d'art du peuple de Beijing, car le style de L'Arrêt ne convenait pas au climat politique d'alors ${ }^{35}$.

L'Arrêt d'autobus, bien loin d'En attendant Godot, cette tragédie qui représente le destin de l'être humain, est plutôt une tragicomédie de la vie ordinaire combinant l'humanisme optimiste et le scepticisme individuel. Encore un point, d'ailleurs, sur lequel Gao et Lu Xun se rejoignent.

\section{L'ombre: le jeu entre la politique et la subjectivité}

\section{Les adieux d'une ombre de Lu Xun: un moi non-lieu}

Lu Xun a écrit une toute petite pièce en prose, Les adieux d'une ombre, recueillie dans La Mauvaise Herbe:

Celui qui dort jusqu'à l'heure où l'heure même s'abolit, il risque bien que son ombre vienne dire adieu à lui en lui adressant ces mots:

Ce que je n'aime pas dans le paradis, je ne veux pas y aller; ce que je n'aime pas en enfer, je ne veux pas y aller; ce que je n'aime pas dans votre futur monde d'or, je ne veux pas y aller.

Pourtant, c'est toi, ce qui me déplaît.

Ami, je ne veux plus te suivre, je ne veux pas y habiter.

Je ne veux pas!

Non, Je n’y tiens pas, je préfèrerais errer dans le non-lieu (无地).

33. GAO, X. J., L'Arrêt d'autobus, op. cit., p. 36

34. VEG, S., «De la marginalité à l'individualité dans les premières pièces de Gao Xingjian », dans L'écriture romanesque et théâtrale de Gao Xingjian, op. cit., p. 147-167.

35. GAO, X. J., 《没有主义 》 (Ne pas avoir de-isme), Taibei, Lianjing, 2001, p. 177-178. 
Je ne suis qu'une ombre, je te dis adieu et je m'engloutirai dans le noir. Les ténèbres sauront m’avaler, à moins que je ne me dissolve dans la lumière.

Mais comme je ne veux pas errer entre ombre et lumière, je préfère encore m'engloutir dans le noir.

Et pourtant, me voilà enfin à compter qui erre entre ombre et lumière. Est-ce l'aube? Je ne sais. Je lève une main couleur de cendre, simulant le geste du buveur qui vide sa coupe. À l'heure où l'heure même s'abolit, je vais entreprendre seule un lointain voyage.

Oh, si c'est maintenant le crépuscule, la noire nuit va m'engloutir. Sinon je me dissoudrai dans l'éclat du jour, si c'est l'aube qui va paraître.

Ami, il est bientôt l'heure.

Me dirigeant vers les ténèbres, je m’apprête à errer dans le non-lieu.

Tu penses au cadeau que je te destine. Mais que pourrais-je t'offrir?

S'il faut vraiment que je te donne quelque chose, ce ne pourront être que ténèbres et néant. Et pourtant je préférerais te laisser seulement les ténèbres, ou encore la clarté du jour qui me permettrait de me dissoudre en toi; je préférerais te laisser seulement le néant, car je ne voudrais en aucune façon occuper la moindre place dans ton cœur. Je souhaiterais ceci, ami -

Je souhaiterais entreprendre seule un lointain voyage dans le noir, sans toi, sans même la compagnie d'aucune ombre. Il n'y aurait que moi, engloutie dans le noir; et cet univers-là tout entier m'appartiendrait. ${ }^{36}$

Ici, l'ombre n'est plus seulement la projection de son maître: elle apparaît comme un vrai personnage. Elle s'adresse avec la voix du premier pronom personnel à son maître " tu ", mais ne témoigne pas, toutefois, d'une subjectivité indépendante. Car la relation entre l'ombre et son maître " tu » est antinomique: l'ombre ne veut plus suivre " tu », refusant la tentation du paradis, l'effroi de l'enfer et aussi l'illusion du " futur monde d'or », mais elle n'existera plus dès lors qu'elle quittera son maître: soit avalée par le noir, soit dissipée dans la lumière.

Les Adieux d'une ombre reflètent certainement la dépression qui a suivi l'illusion brisée par l'échec de la révolution de 1911 et par la réalité de la société chinoise. Lu Xun avait pour un moment accordé

36. LU, X., 《影的告别》 (Les Adieux d'une ombre), nous traduisons sur la base de la traduction de Pierre Ryckmans, dans La Mauvaise Herbe, Paris, Bibliothèque asiatique, 1975, p. 58-59. 
foi à l'idée de continuité progressiste de la société, soutenue par les nouvelles théories du temps linéaire, de l'évolution et de la téléologie historique. En accord avec ces idées, il avait fait coïncider son propre destin avec celui de la nation, l'y avait en quelque sorte intégré. Mais la révolution de 1911 a eu beau proclamer la naissance de la République, rien ne s'est concrétisé, ni l'avènement d'une époque républicaine ni la mise en place d'une démocratie, tandis que le peuple a montré qu'il n'était pas du tout prêt à embrasser l'idée de " politique moderne ». Dans sa célèbre longue nouvelle La Véridique Biographie d'Ah $Q$, Lu Xun décrit, dans un style satirique particulièrement mordant, des portraits de villageois qui, dans un village du sud-est de la Chine, restent aveugles face à la révolution de 1911, la prenant soit pour une monstruosité soit pour une chose à la mode, selon leur "situation politique».

Lu Xun se trouve entre l'idée du progrès et la réalité qui demeure "éternellement » dans l'ombre du « vieux monde ». Dans son article intitulé "Qu'est-il advenu de Nora après son départ? " pour la conférence donnée le 26 décembre 1923 à l'école normale supérieure de jeunes filles de Beijing - dix mois avant la rédaction de La Mauvaise Herbe, il se moque à la fois de lui-même qui se plaisait " à se sacrifier et à souffrir » et du sacrifice en question. Car désormais, pour Lu Xun qui ne cultive plus les idées de sa jeunesse, le sacrifice ne signifie plus un « beau » sacrifice - ce n'est qu'un choix personnel:

Les masses - surtout en Chine - sont d'éternelles spectatrices de théâtre. Si l'on a montré une victime héroïque, elles ont assisté à une tragédie; si la victime était grelottante de peur, elles ont assisté à une comédie. Devant les boucheries d'ovins de Beijing, on trouve souvent quelques individus qui assistent bouche bée, presque satisfaits, au dépeçage d'un mouton. Et l'avantage qu'ils retirent à voir le sacrifice d'un homme n'excède pas celui-ci. D'autant que, quelques pas plus loin, ils auront oublié ce petit moment de joie ${ }^{37}$.

Malgré tout Lu Xun, qui ne s'éloigne jamais de la tradition lettrée, s'obstine à vouloir changer le monde « tout en sachant que c'est

37. LU, X., «Qu'est-il advenu de Nora après son départ? », dans La Tombe, traduit par un groupe constitué par Michelle Loi, Paris, Acropole, 1981, p. 185. 
peine perdue ${ }^{38}$ ». Il se reconnaît malgré lui profondément empreint de la pensée traditionnelle, en laquelle il voit un « poison »:

Mais c'est justement que, moi-même, je souffre de porter sur le dos sans pouvoir m'en délivrer ces vieux monstres dont le poids accablant me suffoque. Et sur le plan idéologique aussi, comment ne serais-je pas touché du poison de Zhuang Zhou [Zhuangzi] ou de Han Fei, parfois laxiste et parfois rigoriste ${ }^{39}$ ?

C'est pourquoi, dans la postface du même essai La Tombe, Lu Xun reprend son projet de "sauver le monde " en l'entrelaçant à la théorie de l'évolution. Il s'oriente vers la position " intermédiaire »:

Peut-être est-ce paresse, mais je me trouve constamment une excuse en pensant que, dans ce monde en évolution perpétuelle, il y a toujours des formes intermédiaires. Entre les plantes et les animaux, entre les invertébrés et les vertébrés, il y toujours des intermédiaires ou plutôt, en termes plus simples, on peut dire que dans la chaine de l'évolution, tout est intermédiaire. Au moment où l'on commence la réforme de la littérature, qu'il y ait des écrivains douteux ${ }^{40}$, partagés entre les deux tendances, cela est naturel, inévitable et même, il faut qu'il en soit ainsi. Leur tâche est de lancer un nouveau cri après la première prise de conscience. D'ailleurs, venus de l'ancien camp, ils connaissent mieux que d'autres ses réalités, si bien que lorsqu'ils se retournent contre lui, il leur est facile, malgré la puissance de l'ennemi, de lui porter un coup mortel. Cependant, ces auteurs sont destinés à passer avec le temps et à disparaître peu à peu. Loin de proposer des buts ou des modèles d'avenir, ils sont tous au plus comme les pierres ou les planches d'un pont ${ }^{41}$.

C'est toute la douleur de Lu Xun: il a en horreur les masses, qui sont pourtant la partie principale de la Chine à laquelle il se consacre. De plus, entre le vieux monde empoisonné et le nouveau

38. On note une attitude similaire chez Confucius, dans Lun yu (Entretiens, XIV, 41); on reprend la traduction de Anne Cheng, Entretiens de Confucius, Paris, Seuil, 1981):

«Zilu vient à passer la nuit à la porte de Pierre.

Le gardien lui demande : D'où venez-vous?

Zilu: De l'école de Confucius.

Le gardien: Ah, je vois, c'est celui qui s'obstine à vouloir sauver le monde tout en sachant que c'est peine perdue?»

39. Ibid.

40. Lu, Xun utilise le mot chinois : « 不三不四 » pour se moquer de lui-même.

41. LU, X., «写在 «坟»后面》 («Postface pour La Tombe »), dans La Tombe, op. cit., p. 337345. 
monde incertain, le passé et le futur sont tous deux douteux, et il n'y a que le présent qui existe véritablement: il est intermédiaire entre le passé et le futur, mais éternel pour le moment, ici et maintenant. Lu Xun dévoile lui-même la signification du "futur monde d'or » de La Mauvaise Herbe, qui provient de L'Ouvrier Shevyryov ${ }^{42}$ de l'écrivain russe M. P. Artzybashev, dont le personnage réfute les idéalistes qui rêvent d'un futur monde d'or en exigeant d'un grand nombre de gens qu'ils souffrent dans le présent pour l'édifier: "Vous réservez ce monde d'or à leurs descendants, mais à eux, qu'avez-vous à donner? » Lu Xun répond lui-même à cette question:

Tout ce qu'on a, dans l'immédiat, c'est l'espoir pour l'avenir, mais le prix en est trop élevé et, pour leur souffrance, à la ressentir plus profondément, à réveiller leur âme pour contempler leur propre cadavre décomposé. En de telles circonstances, seuls le mensonge et le rêve ont quelque chose de grand. Je pense donc que si nous ne trouvons pas d'issue, ce sont des rêves qu'il nous faut, mais pas des rêves sur l'avenir, des rêves sur le présent ${ }^{43}$.

Wang Hui interprète l'anxiété diffuse dans La Mauvaise Herbe comme un état d'esprit auquel il ne manque que d'avoir pu s'intégrer à la pensée existentialiste, notamment celle de Camus et de Sartre ${ }^{44}$. Bien difficile cependant de ranger Lu Xun dans la philosophie existentialiste, du point de vue de l'histoire (l'existentialisme apparaît beaucoup plus tard que Lu Xun) mais aussi des origines doctrinales (la spéculation sur l'Être, le primat de l'ontologie qui forment la base de la philosophie occidentale et de l'existentialisme en particulier ne sont pas les éléments essentiels de la pensée de Lu Xun). Pourtant, ce n'est pas sans raison que Wang indique qu'il y a effectivement chez Lu Xun un sentiment de désert et même d'absurdité indicible qui porte sur l'existence et qui n'apparaît pas auparavant dans la littérature chinoise.

Il n’y a pas simplement l'affrontement entre l'écrivain Lu Xun et le politique Lu Xun, il y va d'abord de la confrontation de Lu Xun

42. LU, X., «工人綏惠略夫》 (L'ouvrier Shevyryov), roman écrit après la révolution russe de 1905, et traduit par Lu Xun en 1921 ; il est intégré aux Euvres Complètes de Lu Xun (鲁迅全 集) XI, Shanghai, Guanghua shudian, $1^{\text {re }}$ édition en 1938, $2^{\mathrm{e}}$ en 1973, p. 595.

43. LU, X., "Qu'est-il advenu de Nora après son départ? », La Tombe, op. cit., p. 179-185.

44. WANG, H., 《反抗绝望》, Beijing, Shalian shudian, 2008, p. 256-260. 
avec lui-même. La douleur devenue plus aiguë et la perplexité plus anxieuse dans Les Adieux d'une ombre dérivent plutôt d'un réveil de la conscience de soi. La fameuse image de "la chambre en fer" que Lu Xun introduisit dans la préface de $\mathrm{Cris}^{45}$, lorsque son ami Qian Xuantong lui avait demandé d'écrire quelques articles pour $L a$ Jeunesse ${ }^{46}$ (《新青年 ») convient aussi pour révéler sa propre situation: « Le plus douloureux, pour l'homme, est de n'avoir nulle voie sur laquelle s'engager au sortir d'un rêve ${ }^{47}$. "

Toujours dans "Qu'est-il advenu de Nora après son départ? ", Lu Xun parle de Nora (l'héroïne d'Une maison de poupée d'Ibsen) dont la fuite n'est qu'un sacrifice inutile pour la liberté. Et la conséquence pour Nora, conclut Lu Xun, est la suivante: « elle n'a le choix qu'entre deux voies: la déchéance ou le retour à la maison ${ }^{48}$ ». Mais en même temps, Lu Xun éprouve de la compassion à l'égard de Nora, même si sa réaction ne sert à rien: " néanmoins, puisque Nora a été réveillée, il ne lui est pas facile de retourner dans le monde de rêves; elle n'a plus alors qu'à s'en aller ».

Lu Xun ne voit-il pas sa propre figure à travers ce personnage typique d'Ibsen? La difficulté de Nora n'est-elle pas effectivement la sienne? Ce qui est «le plus douloureux ", n'est pas seulement qu'il se débat longtemps entre lui-même et le rôle du héros national, entre la liberté personnelle et la responsabilité pour la nation, mais plutôt qu'il se voit lui-même avec ses yeux éveillés tentant de sortir du

45. « La chambre en fer » étant la fameuse métaphore de Lu Xun a paru dans la préface de Cris, traduit par par Michelle Loi, Paris, Albin Michel. 1995, p. 19-20 : «Imagine une chambre avec des murs en fer, absolument indestructibles et sans aucune fenêtre. Imagine là-dedans des gens profondément endormis qui ne vont pas tarder à mourir asphyxiés, mais qui, passant ainsi de l'inconscience de leur sommeil à la dissolution de leur être, ne ressentiront rien de l'angoisse de mourir. Et toi maintenant, tu veux te mettre à crier, à réveiller quelques-uns d'entre eux, ceux qui ont le sommeil le plus léger. Tu obliges cette minorité de malheureux à souffrir les affres de leur mort proche et inéluctable. Crois-tu donc que ce soit leur rendre service? ». Lu Xun explique sa situation, qui lui semble une impasse, en mettant en avant cette métaphore.

46. C'est une revue fondée en 1915 par Chen Duxiu à Shanghai. Elle joue un rôle majeur dans l'introduction des idées occidentales, tant libérales que marxistes, en Chine au début du $X x^{e}$ siècle, avant de devenir une revue du Parti communiste chinois.

47. Lu, X., «Qu'est-il advenu de Nora après son départ? », dans La Tombe, op. cit, p. 179-187. 48. Ibid., p. 180. 
mode de pensée traditionnel; et lorsqu'il s'en prend au « caractère national » de sa plume, il attaque paradoxalement une projection de lui-même.

Dans sa nouvelle Le Sage, l'Idiot et l'Esclave, Lu Xun décrit une figure typique d'esclave qui cherche quelqu'un pour se plaindre du mauvais traitement qu'il a reçu de son maître. Le sage le console avec la compassion qu'il faut, alors que l'idiot se met tout de suite en action: il entre dans la chambre de l'esclave et s'attaque au mur de torchis pour ouvrir une fenêtre. L'esclave appelle les gens pour chasser l'idiot. L'esclave reçoit finalement le satisfecit de son maître, car il a bien protégé sa chambre étouffante.

Takeuchi Yoshimi estime que Lu Xun se représente lui-même à travers cette figure d'esclave qui se plaint d'un mauvais logement mais refuse de le quitter et de se libérer. Ainsi, chez Lu Xun, il y a une tension entre le refus de devenir "soi-même " venu de la tradition, et le refus en même temps d'être un " qui » quelconque en dehors de ce "soi-même ": Yoshimi appelle cette situation " la résistance orientale ${ }^{49}$ " par laquelle, Lu Xun garde son " vrai moi ».

Cette " résistance orientale " est un "non-lieu ». Rappelons que Lu Xun mentionne deux fois "non-lieu» (无地) dans cette petite prose Les adieux d'une ombre: "Non, je n’y tiens pas, je préfèrerais errer dans le non-lieu [...] Me dirigeant vers les ténèbres, je m'apprête à errer dans le non-lieu. " Comme le "non-lieu » est un endroit qu'on trouve nulle part, " errer dans le non-lieu » est, en réalité, un refus total d'aller à aucun lieu possible: l'ombre est à la fois ce qui refuse d'être moi et ce qui refuse d'être l'autre. Pour ainsi dire, elle représente une âme lucide qui est torturée par ce moi du non-lieu. L'ombre révèle une situation où elle est la projection du monde lumineux qu'elle ne peut rejoindre; elle est la vision de l'intériorité qui se mire elle-même mais ne voit que "les ténèbres et le néant "; elle se débat pour quitter son maître mais une fois qu'elle y réussira, elle disparaîtra. L'ombre incarne ainsi la figure de l'irréconciliabilité entre le vieux monde et le monde actuel (les temps modernes); elle se rend compte finalement qu'il est impossible

49. TAKEUCHI, Y., (竹内好) 《近代的超克 》 Jindai de chaoke, Beijing, Shanlian Shudian, 2005, p. 206. 
de se mettre à l'abri ni dans le monde réel ni dans le monde futur imaginaire, ni dans le monde des ténèbres, ni dans le monde de la lumière.

\section{L'Autre Rive de Gao Xingjian: un moi-chaos et un moi lucide}

Où se trouve le moi chez Gao? Les problèmes de la politique et du « moi » qui se jouent dans le tiraillement entre la tradition et l'Occident chez Lu Xun ont leur écho, sur un autre terrain, dans la dernière pièce de théâtre que Gao ait écrit en Chine, L'Autre Rive (1986). C'est une pièce en un acte qui regroupe plusieurs histoires impliquant deux rôles principaux: "l'homme " et "l'ombre ".

La pièce commence par montrer un acteur qui joue avec des cordes: " Je te prie de tenir le bout de cette corde, voilà, entre nous s'établit un lien. Auparavant, moi, j'étais moi, toi, tu étais toi, avec cette corde, toi et moi sommes reliés ${ }^{50}$. " Puis l'acteur montre les façons variées de jouer avec les cordes, métaphore de la relation entre " toi " et "moi ». Il s'agit d'abord d'une confrontation de forces, comme le jeu qui consiste à tirer la corde, un adversaire à chaque bout - il y aura toujours un gagnant et un perdant; mais la relation se modifie selon la manière de jongler, avec les rôles de manipulateur et de manipulé qui n’ont rien de figé. Il y a des liens encore plus complexes: "Si tu tournes autour de moi en me prenant comme centre, tu deviendras mon satellite. Si tu ne consens pas à tourner autour de moi, je pourrai aussi bien tourner sur moimême et en plus crois que c'est vous tous qui tourbillonnez autour de moi. ${ }^{51}$ " Ces liens conduisent à une spéculation philosophique: " En fin de compte, est-ce toi qui tourneras? Ou bien moi? Est-ce moi qui t'encerclerai ou toi qui m'encercleras, ou bien est-ce toi et moi qui tournerons, ou toi et moi qui tournerons en encerclant les autres, ou eux qui tourneront en nous encerclant, toi et moi, ou bien est-ce que nous tous tournerons autour de Dieu? Mais que Dieu existe ou pas, ça, on ne peut le prouver. Peut-être est-ce

50. GAO, X. J., L'Autre Rive, dans Le Quêteur de la mort, suivi de L'Autre Rive et La Neige en août, Paris, Seuil, 2004, p. 55.

51. Ibid., p. 56. 
seulement l'univers qui tourne sur lui-même comme une meule ${ }^{52}$." La métaphore du jeu de corde révèle d'emblée deux thèmes de la pièce: la politique (la relation entre les hommes) et la réflexion sur le moi (notamment sur un " moi " qui se trouve dans des relations complexes).

Ensuite, plusieurs acteurs montent sur scène et jouent avec des cordes de manières différentes, donnant comme une image du réseau des relations sociales. Puis les cordes deviennent une rivière et les acteurs se transforment en foule. Ils sont excités à l'idée d'aller sur l'autre rive, mais ils commencent à se plaindre lorsqu'ils marchent dans l'eau. Finalement, ils arrivent sur une rive (on ne sait de quel côté), mais ils perdent tous la mémoire et se couchent sur le sol. C'est aussi la question que Gao pose dans son titre métaphorique: l'autre rive, s'agit-il de ce qui est au-delà de ce monde? Ou bien, n'est-ce qu'une autre rive par rapport à cette rive-ci?

Une femme apparaît dans l'obscurité, c'est la créatrice du monde; elle réveille les gens un par un, puis les dote du langage. Les gens commencent à se découvrir les uns les autres par le truchement de la langue que la femme leur a apprise. L'« homme " (le protagoniste) apparaît aussi parmi la foule, dont il sort. Cependant, les gens deviennent de plus en plus fous au fur et à mesure qu'ils jouent avec les mots; ils étranglent finalement la femme. Il semble ici que Gao souligne le fonctionnement paradoxal du langage: d'une part, la foule qui a pris conscience de soi grâce au langage, développe en l'utilisant de manière pulsionnelle dans une surenchère verbale malsaine une capacité de violence aveugle et gratuite, puis tue la femme; d'autre part, l'« homme " se distingue de la foule grâce aussi au langage, mais qu'il utilise autrement. L'Autre Rive, pièce métaphorique sur la politique et le moi, fait du langage la clef de compréhension de ces deux univers, et de son bon usage un enjeu crucial.

L'، homme " reproche à la foule son crime, mais, paradoxalement, les gens le reconnaissent comme leur chef. S'enfonçant dans la perplexité, car il ne sait pas que faire, l'" homme " rassemble le groupe et entre par hasard dans une maison où il $\mathrm{y}$ a un joueur de cartes. La foule qui participe immédiatement au jeu est manipulée

52. Ibid. 
dès lors par le joueur de cartes. L'« homme " ne veut pas jouer avec eux, et avertit que le joueur n'est qu'un tricheur. Menacé par le joueur de cartes et par la foule, il se soumet mais s'effondre tout de suite à terre. Au joueur de cartes correspond en quelque sorte la figure du "surhomme " politique intelligent, séduisant et malin, et sachant comment contrôler les esprits.

Au moment où l'" homme " sombre dans le coma, un jeune homme apparaît sur la scène: il figure de la jeunesse de l'« homme ». Le jeune homme rencontre successivement dans son souvenir une jeune fille (son amante), son père et une vieille femme, et par celleci, il tombe dans un autre monde rempli de mensonges, de violence et d'hypocrisie... Le jeune homme s'étonne de ce qu'il a vu et sort de la scène.

L'« homme " sorti de son évanouissement rentre en scène, mais cette fois il y a une ombre, vrai personnage, qui le suit. L'ombre lui fait remarquer qu'il est en train de chercher quelque chose. L'" homme " s'en rend compte, mais il ignore ce qu'il cherche. Il demande à la foule ce qu'elle cherche (l'un répond qu'il cherche une aiguille pour que le chameau puisse passer par le chas; l'autre dit qu'il cherche une bonne position pour s'installer; un bègue dit qu'il cherche une bouche; une fille dit qu'elle cherche l'amour), mais il n'obtient aucune réponse qui vaille pour lui-même. Il est forcé de passer entre les cuisses d'un homme qui surveille tout le monde. Au moment où l'« homme » se faufile entre les jambes de ce dernier, la foule disparaît. Cette scène entre en consonance avec la scène précédente où l'" homme " a dû se soumettre à la force du joueur de cartes. Mais cette fois, l'« homme » gagne sa liberté: « tandis qu'il se faufile, il ramasse une clef sur le $\operatorname{sol}^{53}$ ".

L'ombre rentre en scène aussitôt, assure à "l'homme " que cette clef est précisément ce qu'il cherche. L'« homme » ouvre une porte avec cette clef, et trouve des mannequins en forme de femmes. Il leur tourne les bras et les jambes pour leur donner les poses qui lui plaisent. Puis il les dispose pour former une composition: « il s'enfuit en hâte, saute, roule au milieu de sa propre création, au comble de l'excitation, en criant pour répondre aux cris de sa création. Il se

53. Ibid., p. 110. 
faufile dedans, ses forces déclinent peu à peu et il ne parvient plus à en sortir ». Finalement, il " s'en extrait difficilement en rampant comme un ver exténués ${ }^{54}$.

À ce moment, l'ombre réapparaît et observe l' " homme " dans une attitude très calme, en le suivant. La foule réapparaît aussi et redemande à l'« homme " d'être son guide. Puis le cœur de l'« homme " entre en scène: "Tandis que la foule regarde le cœur, courbé, vacillant, aveugle et sourd qui passe devant elle, l'ombre traîne l'homme par terre, ils sortent en silence. La foule sort lentement en suivant le cœur de l'homme, invisible en fait et pourtant très vieux ${ }^{55}$. " Puis les acteurs entrent par l'autre côté de la scène, ils ne jouent plus leurs rôles et laissent la pièce se terminer en devisant respectivement de leurs affaires quotidiennes.

La pièce coule la vie de l'« homme " dans l'emboîtement en un double récit de deux confrontations qui nourrissent les intrigues et fournissent le dynamisme interne de l'histoire. Il s'agit, d'une part, de l'" homme " en tant qu'individu affrontant la foule, et, d'autre part, de l'« homme » en tant que moi-chaos confronté à son ombre qui s'apparente à celle de Lu Xun, dans son rôle de conscience lucide du moi. Ajoutons que dans cette fable bifide de la société et du moi, la politique est évoque aussi ce qu'elle est chez Lu Xun: omniprésente mais invisible.

Gao rappelle au début de L'Autre rive que la manipulation se réduit à une cruelle alternative: "Si je te tire en passant la corde sur mon dos, tu deviendras une sorte de chien mort. Si, au contraire, c'est toi qui manies la corde, je deviendrai un âne ou un cheval, une sorte de bête de somme que tu guideras ${ }^{56}$. " De même pour Lu Xun: même s'il s'engage dans la politique et les mouvements sociaux ce qui serait le bon côté de la corde, à supposer qu'il y ait du bon à être du bon côté - l'une des raisons de sa douleur et de son désespoir est qu'il sait qu'il ne peut éviter d'être une marionnette, puisque " dans notre société, non seulement les femmes sont souvent les marionnettes des hommes, mais des hommes le sont aussi d'autres

54. Ibid., p. 112.

55. Ibid., p. 115.

56. Ibid., p. 56 . 
hommes, les femmes d'autres femmes, et des hommes sont parfois même les marionnettes des femmes ${ }^{57}[\ldots]$ ».

Gao se méfie de la foule autant et plus que Lu Xun: la tragédie de la femme au début de L'Autre rive a rendu l'« homme » vigilant... Mais à la différence de Lu Xun, Gao ne s'identifie à aucun groupe et prend une position apolitique: pour lui l'homme ne peut être le responsable que de lui-même et ne peut ni être responsable des autres, ni être représenté par les autres.

Ainsi, dans L'Autre Rive, Gao déconstruit le sujet collectif " nous »: ce n'est qu'un trompe-l'œil politique qui transforme, subrepticement, la volonté personnelle en volonté collective. La relation entre la foule crédule et le joueur de cartes tyrannique n'évoque pas seulement le souvenir de la révolution culturelle; elle est la métaphore de tout rapport au pouvoir et désir du pouvoir. Tristement, il n’y a pas que le joueur de cartes qui manipule les autres; l'« homme » qui refuse d'être manipulé s'amuse aussi à ce jeu, dans un élan parodique. Lorsque l'« homme " choisit de passer par l'entrejambe d'un autre homme au lieu de se soumettre à la volonté des autres, il trouve la clef qui était l'objet inconnu/inconscient de sa recherche et entre dans un nouveau monde où il abuse immédiatement de son pouvoir de manipuler les mannequins. Le nouveau monde qu'il découvre, comme l'autre rive où la foule arrive, n'est qu'un monde semblable à ce monde dont il s'est enfui.

Comme il n'y a pas d'" autre rive ", d'au-delà de ce monde où l'on obtiendrait "le salut ", l'« homme " n'est pas plus capable que les autres de s'émanciper de son narcissisme et de son désir du pouvoir. À la fin de L'Autre rive, l'" homme " s'enfui encore, mais cette fois de son propre désir du pouvoir. La fuite lui attribue paradoxalement le sentiment de sacrifice: «Puis l'hiver est arrivé, ce jour-là, il neigeait à gros flocons, tu marchais pieds nus dans la neige pour sentir le froid qui te perçait les os. Tu trouvais que tu ressemblais au Christ, dans ce monde, toi seul souffrais, on ne peut plus solitaire. Tu

57. LU, X., «Qu'est-il advenu de Nora après son départ? », dans La Tombe, op. cit., p. 180. Nous reprenons la traduction du groupe constitué par Michelle Loi, mais modifions quelques mots d'après le texte original chinois. 
te sentais plein d'un esprit de sacrifice, tout en ne sachant pas pour qui tu te sacrifiais ${ }^{58}$."

Le "sacrifice " que l'" homme " éprouve, comme l'ombre le dévoile, est effectivement un non-sens, ou plutôt une "sacrifice imaginaire " qui ne conduit l'» homme » qu'au narcissisme. Ce « sacrifice imaginaire " semble une imitation ironique de celui qu'on trouve chez Lu Xun. Même si le sacrifice, pour ce dernier, est un choix personnel - « on n'a pas le droit de persuader quelqu'un de se sacrifier, pas plus que l'on n'a le droit de l'en dissuader ${ }^{59}$ " — Lu Xun le considère comme une expiation nécessaire ${ }^{60}$. C'est pourquoi, en donnant l'exemple d'Ahasvérus, maudit pour avoir chassé Jésus voulant pendant la marche de la Passion se reposer sous l'auvent de sa maison, Lu Xun se moque (en toute autodérision) de lui mais en même temps considère cette punition comme un sacrifice qui correspond sans doute un sentiment similaire chez lui-même:

Depuis, Ahasvérus ne fait qu'errer sans pouvoir s'arrêter, et il erre encore. Il est pénible de marcher et agréable de se reposer; pourquoi alors ne se repose-t-il pas? Parce que bien que frappé de malédiction, comme on le dit, c'est finalement la marche, et non le repos, qui doit lui convenir. Et c'est la raison pour laquelle il poursuit toujours sa marche folle ${ }^{61}$.

C'est une sorte d'autopunition/sacrifice qui révèle le dilemme de Lu Xun: il contrecarre totalement la culture traditionnelle dans laquelle il se trouve, et il s'implique dans ce qu'il refuse d'être. Comme Ahasvérus est puni de marche sans arrêt, Lu Xun se punit lui-même de combattre sans arrêt. Il se voit comme un soldat qui

58. GAO, X. J., L'Autre Rive, dans Le Quêteur de la mort, suivi de L'Autre Rive et La Neige en août, op. cit., p. 112

59. Lu, X., La Tombe, op. cit., p. 184

60. Takeuchi Yoshimi remarque, lorsqu'il lit Lu Xun, qu'il rencontre toujours quelque chose comme l'ombre qui reste au fond de ses ouvrages, à une même place : « Lu Xun envisagerait lui-même aussi cette ombre en pleine nuit: elle n'est pas Méphistophélès, c'est sûr. Si on cherche un mot pour dire cette ombre, ce sera peut-être le gui (le fantôme) dans la langue chinoise », il indique encore que ce gui implique un sentiment d' " expiation » : « Je trouve que chez Lu Xun, il y avait effectivement la conscience similaire du péché originel dans la religion [...] N'est-ce pas, il y avait un sentiment d'expiation chez lui ? Qu'il ignorait lui-même... »; voir 竹内好 《鲁迅》 (Lu Xun), Zhejiang, Zhejiang Wenyi Chubanshe, 1986, p. 5.

61. Lu, X., La Tombe, op. cit., p. 185. 
se bat contre le monde des ténèbres et "maintient de son épaule la porte entrouverte des ténèbres " afin de laisser les jeunes qui s'avancent vers « les espaces libres et lumineux ${ }^{62}$ ». Ses actions sont pourtant en cohérence avec la morale du "vieux monde ", avec le confucianisme au premier chef: ne faut-il pas se sacrifier afin de sauver les autres, ou bien " avoir avant tout la préoccupation pour le monde (先天下之忧而忧) »? Gao commente ainsi: « l'écrivain Lu Xun est étouffé par le politique Lu Xun. C'est le malheur de la littérature mais pas nécessairement le malheur de Lu Xun lui-même, peut-être son dommage ${ }^{63}$ ".

Sur ce point, Gao se différencie de Lu Xun - plus précisément, il se libère par l'ironie du statut difficile de Lu Xun. Ainsi l'ombre répond à l'« homme ":

En fait, ce que tu éprouves, c'est une sorte de pitié envers toi-même, persifle l'ombre, tu n'as absolument pas envie de finir ainsi, toi qui es si vaniteux ${ }^{64}$.

Il apparaît à Gao que l'idéologie et l'éthique du confucianisme prônant "le sacrifice de soi afin d'atteindre un but sublime " se dévoient dans la réalité dans le mensonge ou la propagande politique. Finalement, " avant de sauver le pays ou le peuple, on a besoin de se sauver tout d'abord. Si l'on ne peut se sauver soi-même, comment est-il possible de sauver les autres ${ }^{65}$ ?".

C'est pourquoi, dans L'Autre rive, l'ombre est plus indépendante de son maître que dans Les Adieux d'une ombre. L'ombre qui apparaît au moment où l'" homme " se perd lui-même (c'est par la conscience de soi que l'" homme " se distinguait autrefois de la masse), est le parallèle de l'« homme »:

L'homme et son l'ombre entrent par un autre côté. L'ombre est vêtue d'habits noirs. L'homme et son ombre ne se voient pas, et se parlent à eux-mêmes, mais leurs gestes et leur pas sont identiques.

L'homme. - Une graine tombe dans la terre...

62. Ibid., p. 174

63. GAO, X. J., «没有主义», Ne pas avoir de-isme, op. cit., p. 28.

64. GAO, X. J., L'Autre rive, dans Le Quêteur de la mort, suivi de L'Autre rive et La Neige en août, op. cit., p. 113

65. GAO, X. J., «没有主义», Ne pas avoir de-isme, op. cit., p. 20. 
L'ombre. - Un enfant est né dans ce monde...

L'homme. - Le vent traverse la forêt...

L’ombre. - Un cheval galope sur la steppe...

$[\ldots]$

Les paroles ou "dialogue " commencent par le contraste de deux voix parallèles. Mais au fur et à mesure, le sujet de leur(s) monologue(s) s'identifie et s'offre au partage: l'« homme " perçoit l'existence de l'ombre:

L'homme. - Une guerre a eu lieu...

L'ombre. - Ce sont les moineaux qui ont été tués...

L'homme. - Les soldats ne se battent pas, ils montent seulement la garde...

L’ombre. - Ceux qui montent seulement la garde méritent tous d'être décorés...

L'homme. - Qui est cet homme qui parle avec moi?

L'ombre. - C'est ton ombre, tes pensées qui parlent...

L'homme. - Tu me suis toujours...

L'ombre. - Quand tu te perds toi-même...

L'homme. - Tu viens alors éveiller mon attention? Accroître encore mes soucis?....

L'ombre. - Que cherches-tu si désespérément?

L’homme. - Tu m'as réveillé! J'ai vraiment perdu quelque chose et je ne sais pas où le retrouver.

L'ombre (sarcastique). - Tu ne sais pas sans doute non plus ce que tu cherches...

L'homme. - Il me semble que c'est... est-ce que tout le monde n'est pas en train de le chercher ${ }^{66}$ ?

L'ombre, une fois sortie du moi, devient la conscience vigilante et lucide qui s'observe et examine ce moi, comme un œil en dehors de lui-même ${ }^{67}$ éclaire en retour le moi-chaos.

Si l'ombre dans Les Adieux d'une ombre apparaît plutôt comme une notion du moi que comme une figure à proprement parler, plutôt comme la concrétisation d'une réflexion entre moi et l'autre que comme une métaphore, elle n'en a pas moins une origine dans la

66. Ibid., p. 98-101.

67. «Un œil en dehors » est une figure venant de la pensée bouddhiste; nous l'interpréterons dans la deuxième partie, au chapitre 2 « Mon regard n'est pas en moi ». 
culture. En effet, Zhuangzi met en scène déjà une notion de l'ombre dans un dialogue entre l'ombre et l'ombre de l'ombre ${ }^{68}$ :

Wangliang (l'ombre de l'ombre ${ }^{69}$ ) interroge l'ombre: « Tout à l'heure tu marchais et maintenant tu t'arrêtes. Tout à l'heure tu étais assise et maintenant tu es debout. Pourquoi n'as-tu pas ta propre conduite? » L’ombre répond: "Est-ce que je ne dépends pas de quelque chose pour être ainsi? Ce quelque chose ne dépend-il pas à son tour d'une autre chose? Je dépends de quelque chose tout comme le serpent dépend de ses écailles et la cigale de ses ailes. Comment saurais-je ce qui est "ainsi" ? Comment saurais-je ce qui est "pas ainsi" ?"

Ni l'ombre ni l'ombre de l'ombre ne sont la métaphore du soi: elles révèlent plutôt la relation entre le soi et l'autre. La question de Wangliang porte sur la volonté indépendante, alors que la réponse de l'ombre ignore ce point qui mettrait la focale sur la conscience de soi et glisse vers la question du "relativisme »: Wangliang dépend de l'ombre, l'ombre dépend de son "maître ", mais on ne sait jamais si le maître dépendra de quelqu'un d'autre. De plus, comment peut-on savoir si l'indépendance vaut mieux que la dépendance? Il est inutile de chercher la cause: toutes sont dans les relations relatives... Ensuite, Zhuangzi nous offre la fameuse métaphore du papillon: Zhuangzi fait un rêve où il est un papillon sans savoir qu'il est Zhuangzi; lorsqu'il se réveille, il ne sait pas s'il était Zhuangzi qui a rêvé qu'il était papillon, ou un papillon qui a rêvé qu'il était Zhuangzi. Si l'on oublie le soi, toutes les relations seront équivalentes.

La question de la volonté du moi, ignorée chez Zhuangzi, est reprise semble-t-il par Lu Xun et Gao, même si l'un est l'admirateur de Zhuangzi et l'autre, son critique: les ombres dans Les Adieux d'une ombre et dans L'Autre Rive s'emploient toutes deux à la recherche de ce qui est en tant que moi, autrement dit, la subjectivité individuelle. Mais l'enquête sur le moi chez Lu Xun est menée dans la vie réelle - dans les combats de la politique, du mouvement social et de la culture; aussi découvre-t-il que le moi est dans un

68. ZHUANGZi, «庄子集释齐物论》(Zhuangzi Jishi), Beijing, Zhonghua Shuju, 1961， p. 110.

69. II y a plusieurs interprétations de Wangliang: pénombre, évanescence de l'ombre, ombre de l'ombre. 
" non-lieu ». Gao qui a eu l'expérience de la révolution culturelle et qui a pris témoignage de la douleur de Lu Xun, se retire pour sa part, se détache peu à peu: sa quête du moi se déploie, surtout après son exil en France, dans l'esthétique.

\section{La figure de Nü Wa}

Dans L'Autre Rive, la femme qui apparaît dans l'obscurité et qui redonne la vie aux hommes semble être la figure de la créatrice divine "Nü Wa » qui, dans le mythe chinois, fait apparaître les hommes:

(Elle hésite, avance dans toutes les directions, comme un filet de fumée légère. Elle examine les hommes couchés sur le sol qui ont perdu la mémoire, se déplace légèrement au milieu d'eux, les touche pour les réveiller un par un. Les gens ouvrent les yeux les uns après les autres et relèvent la tête; ils se retournent, la regardent, ils ont l'air de vouloir dire quelque chose, mais n'y parviennent pas.)

La femme (elle lève la main). Ceci est une main.

La foule (toujours confusément). Ç... Cé... ceç... ceci, çç̧... c’est... u... une... ma... $\operatorname{main}^{70} \ldots$

Dans les textes anciens qui relatent le mythe de Nü Wa, celle-ci crée les hommes par un geste silencieux qui transforme la terre en l'homme, tandis qu'ici la femme redonne la vie aux hommes et leur attribue le langage. Mais, justement, la mort de la femme est causée par le langage par l'action duquel la jalousie et la méchanceté transforment la foule en bande d'assassins:

La foule (ils se tournent les uns après les autres vers elle (la femme), jouant avec les mots). - Que tu es généreuse!

- Que tu es adorable!

- Tu es vraiment ignoble.

- C'est un salaud.

- Tu dis le contraire de ce que tu penses, hypocrite!

- Tu joues toujours un double jeu, fripouille!

- Elle te passe de la pommade, mais au fond d'elle-même elle est jalouse de toi.

- Trop rusée, tu enseignes le langage aux gens pour séduire les hommes!

70. GAO, X. J., L'Autre Rive, dans Le Quêteur de la mort, suivi de L'Autre Rive et La Neige en août, op. cit., p. 62. 
[...]

La femme recule; la foule l'entoure de toutes parts. Ils sont excités par leur langage qui devient de plus en plus pernicieux. Assaillie par les regards de la foule, la femme n'a pus aucun endroit où se cacher. Elle ne peut que chercher secours auprès de l'homme.

La foule (encore plus furieuse). - Débauchée!

- Vipère!

- Sorcière!

- Sans vergogne!

La femme serre fortement l'homme dans ses bras en implorant sa protection. La foule devient hystérique.

La foule. - Regardez, regardez!

- Pouah!

- Débarrassons-nous d'elle!

[...]

La foule se précipite, écarte brutalement l'homme et étrangle la femme dans la panique. Lorsque l'homme s'avance dans la foule et secoue le corps de la femme, celui-ci n’a plus de réaction. La foule est atterrée ${ }^{71}$.

La vigilance s'impose à l'égard du langage et même de la civilisation qui ne peuvent corriger la faiblesse de la nature humaine et, le cas échéant, la propulsent dans la mauvaise direction. Il y a une rémanence de la pensée taoïste dans cette insistance sur la contradiction qui s'introduit entre l'intelligence/la civilisation et l'humain.

Dans une nouvelle de Lu Xun, La Réparation du ciel, Nü Wa est aussi la créatrice des hommes, à cette différence près qu'elle ne reste pas muette. Son destin est identique: son œuvre irréfléchie, les êtres humains, la tue. La Réparation du ciel est en fait une satire qui critique la civilisation chinoise, en adoptant et en réinterprétant le mythe de Nü Wa (issu des anciens documents tel que le Classique des montagnes et des mers et Huainanzi) sous l'angle freudien. En tant que déesse originelle qui crée inconsciemment les hommes, Nü Wa contraste avec ses créatures, comme l'inconscient contraste avec la conscience ${ }^{72}$. Elle ne comprend rien à la langue pédante dont les " petits êtres » se servent, à leurs guerres intestines, ni même au geste " civilisé » de s'habiller. Tandis que les petits hommes qu'elle

71. Ibid., p. 67-69.

72. Lu Xun s'intéressa à la théorie freudienne, notamment à travers les ouvrages de Kuriyagawa Hakusan (㕑川白村), un théoricien littéraire et esthétique japonais, dans les années 1920 . 
crée la considèrent comme sauvagement incongrue: "Votre nudité est indécence et obscénité; elle offense la vertu, sape les rites et enfreint les convenances; c'est une conduite d'animal! L'État a des lois qui sont strictes et elles l'interdisent ${ }^{73}$. " Lu Xun incrimine non tant l'inconscience et la naïveté de la déesse, que la culture confucéenne, et notamment sa la notion de rite (礼). L’hypocrisie se dévoile partout: le " petit homme " qui transmet le message réprobateur de la nudité à Nü Wa "se trouve debout entre les jambes de Nü Wa et regarde celle-ci d'en bas ». Finalement, forcée de réparer le ciel rompu par ses créatures, Nü Wa meurt d'épuisement. Après sa mort, les "petits êtres " profitent tout de suite de son cadavre: "Ils dressent leur campement sur le ventre du cadavre car c'est l'endroit le plus moelleux: ils sont très habiles dans leur choix. Puis ils changent brusquement de ton et s'affirment les seuls héritiers de la déesse; ils corrigent, du même coup, l'inscription en caractères

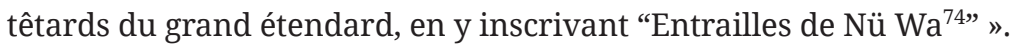

L'impossibilité de communiquer qui sépare Nü Wa et les " petits hommes " fait aussi allusion au fossé qui s'est creusé entre la langue littéraire classique (文言文) et la langue orale (白话文), ellesmêmes symboles de l'ancien monde et du monde moderne. Tenant convaincu de la langue orale comme outil nécessaire de la modernisation culturelle, Lu Xun n'écrit pas La Réparation sans intention: à l'époque, une contre-attaque a cours en faveur de la langue classique et de sa re-substitution à la langue orale, dont la diffusion se trouve menacée. La Réparation du ciel témoigne à n'en pas douter, comme les autres nouvelles de Lu Xun, de son combat contre la vieille civilisation. Car la poursuite de la modernité chez Lu Xun, c'est tout d'abord la liquidation de l'héritage traditionnel.

Ainsi, dans La Réparation du ciel, l'inconscient freudien déguisé en Nü Wa sert d'arme secrète pour attaquer la civilisation chinoise. Nü Wa est une figure inconsciente: elle crée les hommes par hasard sans leur apprendre son langage; elle devient, toujours

73. Lu, X., La Réparation du Ciel (补天), dans Euvres complètes de Lu Xun; nous prenons la traduction de François Jullien dans Lu Xun, écriture et révolution, Paris, Presses de l'École normale supérieure, 1979, p. 23-24.

74. JULLIEN, F., Lu Xun, écriture et révolution, op. cit., p. 24. 
inconsciemment, la victime de la civilisation et de la guerre. Son caractère s'oppose à la culture chinoise qui est trop artificielle et hypocrite, selon Lu Xun.

La femme de L'Autre Rive est, elle, bien consciente et c'est de cette conscience même qu'elle est la victime: Gao a sa modalité propre de critique de la civilisation. Autant dans La Réparation du ciel le conflit entre la langue orale et la langue classique, l'ironie visant la vieille vertu restent enserrés dans la remise en cause de la tradition chinoise, autant, dans L'Autre Rive, la critique porte-t-elle sur la dangerosité de la civilisation (le langage) dans le cadre universel. C'est pourquoi dans La Réparation du ciel Nü Wa et les hommes semblent appartenir à deux mondes différents qui ne peuvent communiquer l'un avec l'autre; tandis que la femme et les hommes, dans L'Autre Rive, se trouvent sur la même "rive ".

En outre, L'Autre Rive accentue le rejet de l'héroïsation par rapport à La Réparation du ciel. Lu Xun montre comment les hommes se battent pour leurs désirs et leurs intérêts; Gao dénonce la violence gratuite qui s'empare des hommes après (et parce) qu'ils ont été civilisés. Témoin de la tragédie, l'« homme " mesure toute l'absurde nocivité de la volonté collective et prend acte de la part du mal dans l'humain; impuissant, il ne peut que crier sa réprobation du crime:

\begin{abstract}
Avez-vous fini? C'est toi, c'est lui, c'est moi, c'est nous tous qui l'avons assassinée! Sur cette autre rive, déserte, elle nous a donné le langage, mais nous n'avons pas su l'utiliser! Ce que nous avons fait est si horrible que nous en sommes nous-mêmes stupéfaits, mais nous sommes si lâches que nous n'osons pas le reconnaître ${ }^{75}$.
\end{abstract}

C'est la première et aussi la dernière fois que l' " homme " prononce un "nous ": désormais, il tentera de se détacher de ce nous et de chercher son propre moi.

Gao s'éloigne donc de plus en plus de Lu Xun dans ce qui touche aux notions d'individu, de tradition et de politique. Mais, en même temps, il ne cesse de rencontrer Lu Xun lorsqu'il remonte à l'origine de la culture chinoise ou oscille entre culture chinoise et culture occidentale. Deux ans après L'Autre Rive, Gao écrit une pièce de

75. GAO, X. J., L'Autre Rive, dans Le Quêteur de la mort, suivi de L'Autre rive et La Neige en août, op. cit., p. 71. 
théâtre mythologique, Chronique du Classique des montagnes et des mers (1989), qui est une adaptation fidèle des anciens mythes sous l'angle psychologique moderne. Au début de la pièce de théâtre, Nü Wa est née en même temps que le monde:

(Soudain, des sons retentissent de toutes parts, des éclairs illuminent la scène, on imagine un tremblement de terre, une éruption volcanique, une pluie de météorites. Dans le chaos, Nü Wa est assise en tailleur, tête baissée, entièrement nue, ses longs cheveux en bataille. La pluie tombe à verse et dévale les pentes des montagnes. Peu à peu elle se transforme en cascades, puis en minces filets d'eau silencieux. Un silence de mort s'installe. Nü Wa lève lentement la tête et se met à murmurer. [...])

Le récitant: (frappant sur son tambour qui rend un son mat.) Mesdames et Messieurs, regardez notre ancêtre à tous, Nü Wa, au milieu de ce chaos. C'est une déesse, naturellement, et non pas une femme ordinaire. Elle est d'une grande beauté malgré sa grande saleté, elle n'a aucune honte et garde sa dignité. On ne sait ce qu'elle dit, on ne sait ce qu'elle chante. Les dieux ont leur propre langage, comment vous et moi, hommes et femmes ordinaires, pourrions-nous les comprendre? (Nü Wa continue à parler et à chanter en se balançant.)

Le récitant: Regardez cette femme, à la fois sauvage et impudique, à la fois tendre et mélancolique, elle chante pour son propre plaisir, sans se préoccuper le moins du monde de sa solitude entre ciel et terre, elle est seule, mais n'est pas dépourvue de joie ${ }^{76}$.

Comparée à la femme de L'Autre Rive, Nü Wa est plus naturelle et primitive; elle parle et chante toute seule pour s'amuser, et non pour être comprise. De même, si elle fabrique les hommes, c'est pour son propre plaisir:

Le récitant: Elle a donné à chacun des mains et des pieds, une bouche et un visage, chacun différent des autres pour qu'on puisse les distinguer. Et à chacun d'eux, sans exception, elle a donné une langue. (Les hommes et les femmes tendent le cou et tirent la langue.)

Le récitant: C'est pour qu'ils puissent manger et boire et distinguer les saveurs, ensuite elle va apprendre, à chacun d'eux, à parler: et tatati, et tatata, et blablabla...

(Chacun se met à bailler ${ }^{77}$ )

76. GAO, X. J., «山海经传》, traduit par Noël Dutrait et Philippe Che, sous le titre de Chronique du Classique des mers et des monts, Paris, Seuil, 2012, p. 16-17.

77. Ibid. p. 19. 
Les hommes, qui ont désormais la langue, pourront « dire leur amour ", " insulter ", " se disputer ", " promettre ", " maudire " ou "mentir »... Nü Wa, en voyant les « mimiques de la foule qui l'entoure ", rit à gorge déployée en frappant dans ses mains ${ }^{78}$. Dans cette pièce, Nü Wa se situe en fait entre la femme de L'Autre Rive et la Nü Wa de La Réparation du ciel: elle n'est plus une femme sacrifiée ni une déesse innocente; elle n'a pas l'intention de sauver les hommes; elle n'est pas non plus inconsciente de sa création. Elle crée les hommes et leur apprend le langage, mais sans en prendre la responsabilité. De plus, elle n'est plus la victime de sa créature, mais une femme désirante qui cherche à satisfaire ses propres désirs. Remarquons la façon dont Nü Wa sort de la scène: « Nü Wa et Fu Xi, tous deux serpents à tête humain, s'enlacent. Puis ils disparaissent peu à peu ${ }^{79}$. » Nü Wa ne correspond-elle pas à la notion du moi libre que Gao recherche? Elle ose montrer ses désirs et les atteindre sans être entravée par la morale, l'éthique et la responsabilité.

78. Ibid. p. 20.

79. Ibid. p. 21. 
DEUXIÈME PARTIE

Y A-T-IL UNE
SUBJECTIVITÉ FLUIDE? 



\section{3 \\ La narratologie ${ }^{1}$ des pronoms personnels et le sujet percevant}

Inventeur de la voix et de l'entendeur et de soi-même. Inventeur de soi-même pour se tenir compagnie. [...] Il parle de soi comme d'un autre. Il dit en parlant de soi, Il parle de soi comme d'un autre.

Samuel Beckett, Compagnie

La beauté est toujours un état éphémère, ce sont tous les instants de passage. Tout ce qui est impossible à fixer, c'est la beauté.

Gao Xingjian, Au plus près du réel

\section{LA PERCEPTION D’UN « AUTRE MOI »}

\section{Les pronoms personnels comme une manière de percevoir}

Gao a commencé à concevoir La Montagne de l'âme, son premier roman, en Chine (notamment pendant un voyage dans le sud-ouest) et l'a achevé dans les deux premières années de sa vie en exil. C'est un roman dans lequel « les personnages sont remplacés par les pronoms personnels, où un parcours intérieur se substitue à l'intrigue, où le style est régulé par l'évolution des sentiments, qui n’a aucune intention de raconter une histoire mais en invente au passage, qui ressemble à un journal de voyage mais se rapproche aussi du monologue ${ }^{2}$ ». Cette œuvre, qui ne saurait être facilement classée,

\footnotetext{
1. Nous empruntons ce terme, issu du formalisme russe et forgé par Gérard Genette, pour éclairer la théorie de la narration chez Gao; nous l'utilisons pour sa signification de " science de la narration » et non par rapport à la sémiologie.

2. GAO, X. J., : «Littérature et 'étude du mystère': à propos de La Montagne de l'âme », dans Le Témoignage de la littérature, op. cit., p. 63-64.
} 
ni parmi les romans picaresques ou d'aventure au niveau de son contenu, ni parmi les romans du flux de conscience au niveau de son style, témoigne pour autant d'une maturité esthétique certaine, dont le marqueur réside dans l'innovation narratologique à laquelle recourt Gao: trois narrateurs, représentés par les trois pronoms personnels « je ", « tu » et " elle ${ }^{3}$ ", combinent leurs interventions tout au long de l'œuvre et lui donnent son ton si particulier.

Dans le roman, " tu ", une figure vagabonde, entend parler par hasard d'un endroit mystérieux appelé Lingshan, et décide d'y aller. Cette voix « tu » entame le premier chapitre en partageant le déroulement de son aventure avec une autre voix — « elle " - , dans un monde spirituel où l'histoire de "tu/elle " ne respectera pas l'ordre temporel et le déplacement spatial ordinaires, mais suivra le courant psychologique qui entrelace les souvenirs personnels, les contes populaires, les légendes historiques, les mythes jadis perdus et les récits folkloriques. Ces deux voix, " tu » et " elle ", font contraste avec la troisième voix — « je » - en laquelle s'incarne sans doute l'identité de l'auteur lui-même: écrivain de Beijing, « je " part pour le sud-ouest de la Chine afin de s'éloigner de polémiques étouffantes.

3. Les écrivains français contemporains, notamment Michel Butor, Marguerite Duras, Louis Aragon, Jacques Prévert, Henri Michaux, André Malraux ont influencé profondément Gao par leur façon d'employer les pronoms personnels. Par exemple, le « tu » qui traverse toute la Modification de M. Butor, ou encore «je», «tu » et «il » qui alternent dans Les Communistes de L. Aragon. Noël Dutrait, le premier traducteur de la plupart de ses œuvres, a raconté une anecdote: Iorsqu'il a traduit La Montagne de l'âme, Gao lui avait recommandé de lire les romans de ses auteurs préférés (voir l'article de Noël Dutrait "Traduire Gao Xinjian, le traducteur comme interface de l'interface », dans «Le Choix de la Chine d'aujourd'hui : entre la tradition et l'Occident », colloque tenu à l'ENS LSH de Lyon les 26-27 novembre 2004. http://w1.ens-Ish.fr/colloques/chin...). Ainsi, on retrouve chez Gao certains procédés typiques de Duras, de Perec : la répétition de « elle dit... » dans L'Amant de Duras par exemple, ou l'alternance de deux récits par chapitre, l'un racontant une histoire réelle ou autobiographique, l'autre traitant d'un voyage spirituel dans $W$ ou le souvenir d'enfance de Perec. Dans un entretien avec Annie Curien (dans La Nouvelle Revue française, janvier 2001, n556, p. 169-177), Gao dit ceci : « J'apprécie surtout Georges Perec, dont j'aime la simplicité, le raffinement, la capacité à construire. Ainsi, L'Homme qui dort, qui existe d'ailleurs aussi en film. En commun, selon moi, avec mon écriture : une orientation, une attention à l'oreille, une poésie, le quotidien, une absence de lyrisme. Perec énumère les choses, rien n'intervient, et le texte est là. Perec est aussi un grand inventeur des formes, et je pense notamment à $W$ ou le Souvenir d'enfance. Pour la langue, je recherche une souplesse, comme les mathématiques peuvent en produire [...] ». 
Il y a ainsi deux voyages simultanés, l'un dans le monde spirituel et l'autre dans le monde réel. "Lingshan » est le mot clef dans l'histoire de « tu », la " vérité » est le but dans le cheminement de « je ».

Dès lors, les récits de " tu/elle " marqués par le dialogue et ceux de « je " tenus par la voix narrative alternent systématiquement, chapitre par chapitre, à travers toute l'œuvre. Juste, l'ordre de l'alternance est interrompu dans les chapitres 31 et 32, où les récits « tu/ elle " apparaissent deux fois successivement, occupant dès lors les chapitres pairs; les récits de " je " se retrouvent ainsi dans les chapitres impairs.

À partir du chapitre 32 également, les récits de "tu " ne se limitent plus aux contes populaires ou légendes historiques, mais font aussi référence à ses propres souvenirs et à ceux de sa famille; de même, par une sorte de chiasme, ce que " je » éprouve, n'est plus seulement situé dans un monde réel, mais se place aussi dans un monde intérieur et fantastique ou dans les histoires lointaines. La voix " tu " et la voix « je " se rapprochent de plus en plus, réunissant de la sorte le passé et le présent du moi. Dans les deux derniers chapitres, où " elle " a complètement disparu, il n’y a plus de dialogue ni de recherche: seule subsiste la perception sensible de " tu " et de " je ». Celle-ci apparaît sous forme de rêve pour " tu »: " tu " remonte le glacier en parcourant tours et détours, entre la vie et la mort. Dans ce monde cristallin, une musique silencieuse mais qui "remplit l'air » le ramène vers le " paroxysme, orgasme purement cérébral ${ }^{4}$ " puis «tu » se réveille et réalise qu’il ne se trouve que dans sa chambre (de Beijing?). Alors que pour « je » du dernier chapitre, le monde se fait métaphorique et allégorique: « je » rencontre le dieu à l'apparence de grenouille qui lui parle en clignant de l'œil, et dans ce lieu d'un grand calme, rempli par la neige, " je " comprend que ce qu'il prétendait comprendre est finalement ce à quoi il n'a rien compris.

Le Livre d'un homme seul, le deuxième roman d'autofiction mais aussi le dernier roman de Gao, possède la même structure analogique d'alternance des récits que La Montagne de l'âme, si ce n'est

4. GAO, X. J., La Montagne de l'âme (traduit du chinois par Noël et Liliane Dutrait), Paris, l'Aube, 1995, p. 666. 
que le rapport triple de " je », " tu » et " elle » est remplacé par la relation jumelle de " tu » et « il »; quant à « je », il disparaît complètement. Pourtant, ce à quoi se réfèrent les pronoms personnels est plus clair: « tu » incarne le moi dans le présent, « il » est le moi dans le passé - notamment à l'époque de la révolution culturelle. " Elle ", l'interlocutrice de " tu » dans La Montagne de l'âme, est remplacée par des femmes concrètes dotées de leurs noms propres et qui ne représentent plus l'ombre de " tu ». L'alternance entre les chapitres de «tu » et les chapitres de «il » est similaire à celle relevée dans La Montagne de l'âme: on a deux histoires en déroulement parallèle dans leurs propres temps et espaces, mais qui se croisent dans le souvenir. Marguerite qui, fille d'un père allemand et d'une mère juive, se débat dans ses tensions identitaires, mène " tu " à se souvenir de l'expérience de « il ", qui mêle les souffrances de la révolution culturelle et le sentiment honteux d'y avoir participé. Le rythme d'alternance des chapitres est interrompu plusieurs fois dans $L e$ Livre d'un homme seul. Les chapitres de « il " - le souvenir du passé — submergent ceux de " tu » - le moi du présent: du chapitre 27 au chapitre 45 , il n'y a que trois chapitres de « tu ». Puis « tu » entre dans le monde du passé de «il ", et fait sienne à la perspective de ce dernier (dans les chapitres 46 et 48); « il » vient aussi dialoguer avec " tu » (dans les chapitres 56 et 60). À la fin du roman, « tu » et « il » se disent adieu: "Les bouddhistes parlent de nirvana, les taoïstes de passage à l'état d'immortel, mais lui dit simplement que tu n'as qu'à le laisser partir. Personne ne peut délivrer personne de sa souffrance, alors laisse-le partir 5 . » Mais, en réalité, «tu » ne se séparera jamais d'avec « il » qui est sa projection temporelle. Dans le dernier chapitre, « il " paraît dans le moment présent où « tu " se trouve à Perpignan:

Le moment à présent, à Perpignan, c'est après le petit déjeuner: des voitures passent sous ta fenêtre en se reflétant sur les boules blanches des lampadaires de la rue, tu n'as pas le temps de les distinguer, leurs ombres disparaissent en un clin d'œil. Il existe dans ce monde tant d'ombres et de lumières qui risquent aussi de s'évanouir. Tu t’amuses avec celles de cet instant-là, il te faut aussi considérer ce

5. GAO, X. J., Le Livre d'un homme seul, (traduit du chinois par Noël et Liliane Dutrait), Paris, l'Aube, 2000, p. 551. 
« il » comme une ombre avec laquelle tu joues, et tu risques d'être un peu surpris, ah, cette ombre qui s'évanouit en clin d'œil ${ }^{6}$ !»

Beaucoup de chercheurs ont remarqué cette narratologie si particulière, inaugurée dans La Montagne de l'âme et développée encore dans Le Livre d'un homme seul. Mabel Lee montre comment les trois pronoms personnels se rattachent à la pensée d' « auto-observation à l'attitude froide " de $\mathrm{Gao}^{7}$; Jin Siyan considère cette narration du pronom personnel comme jeu de miroir entre " je », " tu », « il/ elle » déterminant une relation ambiguë entre les narrateurs, relation dont la prégnance fait que le récit réel et l'imaginaire ne se distinguent plus ${ }^{8}$; Kwok-kan Tam remarque que ce recours narratologique assure une continuité de la subjectivité à travers le courant de langage, notamment dans Le Livre d'un homme seul; ${ }^{9}$ Gang Gary $\mathrm{Xu}$ estime que Gao utilise les pronoms personnels comme la métaphore d'une morale introspective, mais aussi comme la métaphore de la relation entre le narrateur lui-même et autrui ${ }^{10}$; Annie Curien montre que l'usage des pronoms personnels donne d'emblée un rythme musical à la narration, qu'il scande de rimes intérieures ${ }^{11}$. Autant de critiques pertinentes et variées des points de vue littéraire, psychologique, philosophique ou musical... En revanche, une dimension très importante n'a guère retenu l'attention des commentateurs, celle de la perception dans ses divers registres (sujet percevant, perception entre pronoms personnels, perception

6. Ibid., p. 554-555.

7. LEE, M., «Pronouns as Protagonists: Gao Xingjian's Lingshan as Autobiography », dans China Studies, n 4, 1999, p. 165-183; voir aussi «Pronouns as protagonists: on Gao Xingjian's théories of narration », dans Soul of Chaos, édité par Kwok-Kan Tam, Hong Kong, The Chinese University Press, 2001, p. 235-256.

8. JIN, S. Y., L'écriture subjective dans la littérature chinoise contemporaine: devenir je, Paris, Maisonneuve \& Larose/Espace du temps présent, 2005, p. 214-218.

9. TAM, K. K., «Language as Subjectivity in One man's Bible », dans Soul of Chaos, édité par Kwok-Kan Tam, op, cit., p. 293-310.

10. XU, G. G., "My writing, your pain, and her trauma: pronouns and (gendered) subjectivity in Gao Xingjian's Soul Moutain and One man's Bible», dans Modern Chinese literature and culture, vol. 14, 2002, n² 2, p. 99-129.

11. CURIEN, A., «Solitude et échos vocaux dans l'écriture de Gao Xingjian », dans L'écriture romanesque et théâtrale de Gao Xingjian, Paris, Seuil, 2006, p. 169-187. 
visuelle, etc.) et notamment sous l'angle d'une convergence sur ce point de la pensée contemporaine et de la culture source originelle.

Gao peut bien déclarer que le roman n'exprime que l'expérience personnelle de l'auteur, cette " expérience de la vie » ne laisse pas d'être influencée par la société et la culture dans lesquelles baigne ou a baigné l'auteur: comme le souligne Wayne $\mathrm{C}$. Booth, un roman implique toujours " certaines théories concernant l'existence, certaines métaphysiques ${ }^{12}$ ". Bien que les romans de Gao mobilisent plutôt une pensée non systématisée et un "non-isme ", on y décèle, malgré tout, les traces de la pensée de son époque, occidentale aussi bien qu'orientale, comme de sa culture originelle. Les idées forces qui le poussent à aller de l'avant sont proches de celles de la phénoménologie et de son mot d'ordre de la réduction au phénomène, et s'entrelacent avec celles de la psychanalyse (de Freud) et du bouddhisme chán (de Huineng ${ }^{13}$ ), ou encore avec celles du taoïsme (notamment de Zhuangzi). Son style très personnel fait discrètement chatoyer, au fil de ses ouvrages, les reflets de l'ensemble de ces pensées.

Quelques paragraphes du chapitre 52 de La Montagne de l'âme sont souvent mentionnés par les critiques: ce sont ceux où Gao Xingjian dévoile lui-même les significations de " je ", de « tu » et de " elle », et s'explique sur leurs rapports mutuels:

Tu sais que je ne fais rien de plus que me parler à moi-même pour distraire ma solitude. Tu sais que ma solitude est sans remède, personne ne peut me soulager, je ne peux avoir recours qu’à moi comme partenaire de mes discussions.

Dans ce long monologue, " tu " est l'objet de mon récit, en fait c'est un moi qui m'écoute attentivement, « tu » n'est que l'ombre de moi. Pendant que j'écoutais attentivement mon propre «tu», je t’ai fait créer " elle ", parce que tu es comme moi, tu ne peux supporter la solitude, tu dois aussi trouver quelqu'un à qui parler.

$\mathrm{Tu}$ as donc eu recours à " elle » de la même manière que j'ai eu recours à « tu ».

« Elle» dérive de « tu » et, en retour, confirme mon moi ${ }^{14}$.

12. Bоотн, W. C., The Rhetoric of Fiction, Chicago \& London, The University of Chicago Press, 1961, p. 79.

13. Huineng (慧能, 638-713) fut le sixième maître de l'école chán.

14. GAO, X. J., La Montagne de l'âme, op. cit., p. 421. 
On retrouve à n'en pas douter quelque chose, mutatis mutandis, de la topique freudienne " conscient-préconscient-inconscient " dans ce « je-tu-elle » de Gao: «tu » est l'ombre de « je »; partie qui tend à échapper à la conscience, il équivaut au préconscient, tandis que " elle ", l'ombre de l'ombre " tu », est comme l'inconscient qui rencontre " tu » (préconscient) mais reste en parallèle de « je » (conscient). Par exemple, le souvenir de " elle " évoque souvent le souvenir de "tu », qui a lui-même des liens avec le passé de " je », réel ou imaginaire; mais il n’y a pas d'intersection directe entre le souvenir de " elle " et celui de « je » : comme chez Freud, l'inconscient est inaccessible au conscient, et le préconscient incarne la partie de la mémoire qui se trouve à la périphérie du champ de la conscience. Pourtant, à la différence de ce qui se passe chez Freud, où le refoulement ou la sublimation de la pulsion sexuelle expliquent la motivation intrinsèque venant du préconscient ou de l'inconscient, chez Gao, c'est dans l'activité perceptive, sens interne et sens externes confondus, que réside l'impulsion originelle de " tu » et " elle », voire de « je ». Ainsi, " tu » ne désigne pas un esprit moral qui implique les interdits parentaux et sociaux (complexe d'CEdipe, par exemple), comme le surmoi freudien; de même, « elle " ne représente pas non plus une instance pulsionnelle originelle comme le ça régi par le principe de plaisir. La création des personnages « je ", " tu » et " elle " dans La Montagne de l'âme se fonde sur un principe esthétique plutôt que sur un principe éthique, sur l'expérience sensible et perceptive plutôt que sur l'expérience cognitive.

Il y a en effet deux tendances contradictoires dans cet usage des pronoms personnels, concernant le moi. La première est celle d'une convergence du moi, selon une logique " centripète »: " je ", « tu » et " elle " apparaissent comme trois silhouettes du moi qui dépendent l'une de l'autre, et représentent les différents niveaux de la perception du moi. Comme Gao l'explique lui-même, les trois personnes alternent successivement dans La Montagne de l'âme, " pour exprimer leurs perceptions sur un même sujet ${ }^{15}$ ". S'agit-il alors d'une

15. GAO, X. J., «Littérature et 'étude du mystère': à propos de La Montagne de l'âme », dans Le Témoignage de la littérature, op. cit., p. 57. 
autoprojection, d'une sorte d'égocentrisme diffracté ? Comment les trois perçoivent-ils " un même sujet » ?

La seconde tendance serait celle de l'autodivergence du moi: dès lors que " je ", " tu » et " elle " représentent trois divisions du moi, incarnent trois sujets indépendants pris dans leurs mondes respectifs, leurs perceptions ne sont plus cohérentes et ne peuvent se rabouter en un "même sujet». Le moi se scinde et s'étend, ou pour mieux dire se distend vers l'extérieur, avec des retours de coïncidence, quand la mémoire et le langage réunissent les trois parties en quelque occasion. Ce «moi » ainsi divisé en plusieurs personnages pourvus de leurs propres perspectives, comment représenter sa perception du moi - celle qu'il a et celle qu'on en a? Dans la mesure où « je » a complètement disparu dans Le livre d'un homme seul, qui sera désormais le sujet de la perception?

Pour départager, s'il se peut, ces deux tendances opposées à l'égard du moi, toutes deux rendues concevables et mises à l'œuvre par cette narratologie des pronoms personnels à laquelle Gao a sacrifié le sujet banal et bien plus fictif, en réalité, des récits ordinaires et fragmentaire, il faut affronter impérativement deux questions: " qui perçoit? » et « comment percevoir? ».

\section{L'autre moi à percevoir}

Lorsque Merleau-Ponty interprète la perception, il s'interroge d'abord sur la pensée objective qui ignore longtemps le vrai sujet du sentir: " [La pensée objective] se donne le monde tout fait, comme milieu de tout événement possible, et traite la perception comme l'un de ces événements ${ }^{16}$. " Ainsi, selon Merleau-Ponty, lorsqu'un philosophe de la pensée objective décrit les sensations et leur substrat, il ne va pas s'apercevoir « qu'il perçoit lui-même, qu'il est sujet percevant et que la perception telle qu'il la vit dément tout ce qu'il dit de la perception en général ${ }^{17}$ ». Rappelons la fameuse phrase: «Si je voulais traduire exactement l'expérience perceptive, je devrais

16. Merleau-Ponty, M., Phénoménologie de la perception, dans Maurice Merleau-Ponty, Euvres, Paris, Gallimard (Quarto), 2010, p. 896.

17. Ibid. 
dire qu'on perçoit en moi et non pas que je perçois ${ }^{18}$. " Pourtant, cet " on " anonyme paraît ambigu: de quoi s'agit-il exactement? d'autrui? de ce monde? ou bien de soi-même?

Il semble qu'il ne s'agisse ni de l'un ni de l'autre. Merleau-Ponty assure d'abord que nos corps ne sont pas le vrai sujet du percevoir. Bien que toute sensation n'ait pas lieu sans une adaptation de nos corps, elle comporte un germe de "rêve " ou de "dépersonnalisation » - celle-ci est comme ce que nous éprouvons dans une sorte de stupeur " où elle nous met quand nous vivons vraiment à son niveau ${ }^{19}$ ". De plus, l'activité de la perception " se déroule à la périphérie de mon être, je n'ai pas plus conscience d'être le vrai sujet de ma sensation que de ma naissance ou de ma mort ${ }^{20}$ ". Il y a en effet deux moi qui ne se distinguent pas par la dualité corps/âme, mais comme l' « être » de la connaissance et l' « être » de l'expérience. De fait, " le sujet qui en a l'expérience commence et finit avec elle, et comme il ne peut se précéder ni se survivre, la sensation apparaît nécessairement à elle-même dans un cadre de généralité, elle vient d'en deçà de moi-même, elle relève d'une sensibilité qui l'a précédée et qui lui survivra, comme ma naissance et ma mort appartiennent à une natalité et à une mortalité anonymes ${ }^{21}$ ». C'est " par la sensation ", écrit Merleau-Ponty, " que je saisis en marge de la vie personnelle et de mes actes propres une vie de conscience donnée d'où ils émergent, la vie de mes yeux, de mes mains, de mes oreilles qui sont autant de Moi naturels ${ }^{22} "$.

Ainsi, il y a un vrai sujet du sentir en tant qu' " autre moi » : il est en $m o i$, fonctionne en dépendant de mes organes, mais en même temps dépasse mon corps. En d'autres termes, cet autre moi me fait éprouver l'expérience de la sensation, mais ne me fait pas éprouver l'auteur de cette perception. Voici l'expérience de Merleau-Ponty:

Chaque fois que j'éprouve une sensation, j'éprouve qu'elle intéresse non pas mon être propre, celui dont je suis responsable et dont je

18. Ibid., p. 905.

19. Ibid.

20. Ibid.

21. Ibid., p. 905-906.

22. Ibid. p. 906. 
décide, mais un autre moi qui a déjà pris parti pour le monde, qui s'est déjà ouvert à certains de ses aspects et synchronisé avec eux ${ }^{23}$.

« Tu » et " elle » dans La Montagne de l'âme apparaissent en quelque sorte comme cet " autre moi » chez Merleau-Ponty: ils se présentent comme la modalité d'une " existence générale » - si l'on emprunte encore l'expression à Merleau-Ponty — qui est « déjà vouée à un monde physique et qui fuse à travers moi sans que j'en sois l'auteur ${ }^{24}$ ". Comme cet autre moi perceptif existe avant le moi intellectuel, les chapitres de "tu/elle " se situent avant ceux de « je » dans la première partie du roman - la perception avant la conscience.

Quoique « tu » et « elle » soient respectivement l'ombre de « je » et l'ombre de l'ombre de « je ", ils ne possèdent pas simplement une personnalité indépendante, mais aussi une capacité de féconder: " tu » est la création de "je » mais en même temps le créateur de " elle ». Quant à cette dernière, elle agit réciproquement sur « je »: « Elle » dérive de « tu » et, en retour, confirme mon moi ». De ce point de vue, la limite entre les ombres et leur " maître " est ambiguë, ils peuvent se remplacer l'un par l'autre: "Je ne serais que ton ombre et toi, tu deviendrais mon corps réel. " Aussi la relation « je »/« tu » peut être renversée: "Si, à ma place, tu m’écoutais attentivement, je deviendrais l'incarnation de ton désir ${ }^{25}$. " Cependant, cela ne veut pas dire que « je » devienne l'objet perçu de « tu/elle ». Si « je », « tu » et « elle " n'incarnent que le même sujet moi, alors quand « tu » dialogue avec « je », tous les deux sont à la fois les sujets perceptifs et les objets perçus de l'un par l'autre. Autrement dit, il n'y a pas un renversement entre sujet et objet, mais plutôt une «indifférence " ou une indistinction à l'égard du dualisme sujet/objet.

Or, « je ", " tu » et " elle " sont en même temps des personnages indépendants - la perception de « je », celle de " tu » et de " elle " se relient dans un système de perceptions inter-moi(s). Ainsi, « tu » et « elle » éprouvent la perception du moi, mais n’appartiennent pas à l'entité moi: ils représentent à la fois les personnages normaux et

23. Ibid.

24. Ibid.

25. Ibid. p. 424. 
la perception elle-même du moi. En d'autres termes, «tu » et " elle " révèlent une fluidité de la perception du moi qui n'est pas entravée par les limites du temps, de l'espace, des organes, et même du sujet: il y a une dimension ouverte entre la perception et le sujet.

Voici un beau passage décrivant le paysage du coucher de soleil dans le premier chapitre de La Montagne:

La boule pourpre dégage plus encore de sérénité, mais, en descendant au creux du vallon, elle porte une certaine séduction dans sa gravité: il y a les sons $\mathrm{s}^{26}$. Tu en entends un, difficile à saisir, qui se met à résonner au fond de ton cœur et se répand progressivement, tressaille un peu, comme sur la pointe des pieds, s'échappe et disparaît dans le paysage noir de la montagne, emplissant les cieux de la brume du crépuscule ${ }^{27}$.

Il y a une perception panoramique qui circule librement entre les sensations de la vision et de l'ouïe. Même si le sujet du sentir est absent dans la première phrase, la sensation organique, passant de visuelle à auditive, reste dans ce même sujet " tu »: l'image du coucher de soleil à l'extérieur évoque les sons à l'intérieur de son corps. Pourtant, lorsque " tu » apparaît dans la deuxième phrase, un glissement du personnage " tu » aux sons, puis à la perception elle-même se produit: les sons que " tu " éprouve au fond du cœur, s'identifient à la sensation de « tu " et deviennent le sujet du sentir. Les sons ressortent de l'intérieur du corps et éprouvent un tressaillement, puis emplissent " les cieux de la brume du crépuscule ». Dans ce trajet de l'extérieur (le coucher du soleil) à l'intérieur (les sons au fond du cœur) qui ensuite retourne à l'extérieur, il n'y a pas seulement un passage entre les sujets du sentir, mais aussi de la manière de percevoir: de la vue à l'ouïe, puis à la sensation tactile, et finalement retour à la sensation visuelle.

26. Nous modifions un peu la traduction de cette phrase dans la version de Noël et Liliane Dutrait, bien que leur traduction soit certainement plus belle du point de vue de la langue française: "La boule pourpre dégage plus encore de sérénité, mais, en descendant au creux du vallon, elle porte une certaine séduction dans sa gravité. Et puis il y a les sons ». Selon la version originale chinoise: 《坐入山谷那赤红的一轮越发安详, 端庄中又带 点妩媚, 还有声响 》, les sensations visuelles et auditives sont simultanées.

27. GAO, X. J., La Montagne de l'âme, op. cit., p. 18. 
Voici encore un autre passage, concernant la perception de « elle »:

Elle dit que des tentures épaisses pendent, les unes après les autres. Quand on avance parmi elles, on a l'impression de glisser. En écartant doucement les tentures de velours vert foncé, et en se faufilant entre elles, on ne voit personne, il n'y a pas un bruit, le tissu absorbe les sons, il n’y a qu'une musique, parfaitement pure, tamisée par les tentures, une musique qui s'écoule doucement, venant d'une source limpide pleine de douceur; là où elle passe, apparaît une faible lumière.

Elle dit qu'elle avait une tante très belle qui se déplaçait souvent devant elle dans la maison, vêtue seulement d'un minuscule soutiengorge et d'une toute petite culotte. Elle avait toujours eu envie de toucher ses cuisses brillantes, mais jamais elle n’avait osé. [...] Elle était actrice et nombreux étaient les hommes qui la poursuivaient de leurs assiduités. Elle disait toujours qu'ils l'importunaient terriblement, mais en fait elle aimait cela. Plus tard, elle s'était mariée à un officier qui l'avait étroitement surveillée. Si elle rentrait un peu en retard, il la questionnait et même la battait parfois. Elle dit qu’à l'époque, elle n'avait pas compris pourquoi sa tante ne l'avait pas quitté, ni comment elle avait pu supporter cette humiliation.

Elle dit encore qu'elle avait aimé un professeur, son professeur de mathématiques, oh, il ne s'agissait que du sentiment d'une petite fille ${ }^{28}[\ldots]$

Gao n'adopte pas la manière de procéder traditionnelle de l'Occident, qui s'appuie sur la constatation que le sujet regarde dans telle direction, observe selon telle perspective, afin d'exprimer la vue extérieure et l'expérience intérieure que ce même sujet éprouve. En revanche, il n'y a pas de point « focal » de la perspective du narrateur " elle » : lorsque « elle " fixe son regard sur un objet perçu, " elle " lance en même temps une vision sur elle-même. Le glissement dans le cercle de la sensation visuelle-auditive-visuelle, est également marqué par la transmission de la vision extérieure à l'ouïe intérieure (des tentures aux sons), puis à la vision auditive extérieure aussi bien qu'intérieure: la musique lumineuse. Cette transmission se passe silencieusement, précisément comme ce qui glisse entre les tentures de velours, et se dirige vers la sensation

28. Ibid., p. 218. 
d'amour. De plus, avec le pronom personnel « elle " qui, à lui seul, désigne deux personnes (" elle " et la tante de " elle»), une ambiguïté entre les deux personnes émerge. Or, cette ambiguïté explique exactement le désir qu'a " elle » de devenir comme sa tante. Le désir est prouvé par ce que " elle » raconte de sa propre histoire d'amour fantastique: c'est le sentiment d'une petite fille qui ne sera jamais atteint.

\section{La fluidité de la perception: révélatrice du désir}

Il semble que la perception de " tu " et de " elle " coule comme l'eau qui coule, dérive ou conflue mais ne demeure jamais longtemps fixée sur un certain sujet. Grâce à l'emploi des pronoms personnels impliquant une dénotation subjective équivoque, leur perception apparaît anonyme et ouverte au monde extérieur des autres, mais aussi au monde intérieur situé entre eux-mêmes. Aussi, comme l'eau fluide sans forme mais en même temps formée et reformée sans cesse par le trajet qu'elle emprunte, la perception de " tu » et de « elle » coule à travers les sujets percevants, par lesquels elle transmet entre les sujets ou transforme selon les sujets, en montrant leurs processus psychologiques, atteignant jusqu'au fond de leur inconscient, et révélant leurs désirs dissimulés. Prenons quelques paragraphes du chapitre 9 comme exemple:

Elle dit qu'elle n'ose pas regarder vers le bas, tu dis que tu sais très bien qu'elle a peur.

Peur de quoi?

Peur de l'eau.

Elle se met à rire, mais tu perçois que son rire est un peu forcé.

Tu n'oserais pas sauter, dis-tu en marchant volontairement tout près du bord. Au bas de la digue, l'eau de la rivière bouillonne.

Et si je sautais? dit-elle.

Je plongerais pour te sauver. Tu sais qu'en parlant ainsi tu gagneras ses faveurs.

Elle dit qu'elle a un peu le vertige, puis elle ajoute que c'est très facile de sauter, qu'il suffit de fermer les yeux, que c'est la manière de mourir qui fait le moins souffrir, et qu'en plus c'est enivrant. Tu dis que dans cette rivière a déjà sauté une jeune fille comme elle, venue de la ville elle aussi. Elle était plus jeune, encore plus simple. Tu ne veux pas dire que, elle, est particulièrement compliquée, mais que les gens d'aujourd'hui ne sont ni plus ni moins bêtes que ceux d'autrefois, et 
qu'autrefois, ce n'est pas si loin. Tu dis que c'était par une nuit sans lune, la rivière semblait encore plus profonde. [...] Au lever du jour, les occupants d'un radeau l'avaient retrouvée sur un banc de sable à trente lis d'ici. Elle était torse nu, ses habits avaient dû s'accrocher aux branches d'un arbre dans un coude de la rivière. [...]

Tu m'écoutes? demandes-tu.

Continue, répond-elle à voix basse.

Dans le passé, il y a toujours eu des morts ici, des enfants, des jeunes filles. [...] On raconte encore qu'à minuit on voit apparaître là une femme fantôme revêtue d'un habit blanc qui chante une chanson dont on ne perçoit pas clairement les paroles. On dirait aussi bien une chanson enfantine paysanne que la complainte d'un mendiant. Il ne s'agit bien sûr que de superstitions, les gens se font souvent peur à eux-mêmes. [...] Puis, quand tu lui parles d'une autre jeune fille, une jeune paysanne, elle t'écoute attentivement. Quand tu dis qu'elle est morte, victime d'une injustice, dans cette rivière, quand tu expliques ce que disent les gens, elle hoche la tête. Hébétée, elle t’écoute ${ }^{29}$ [...]

Le regard jeté sur l'eau évoque la peur pour « elle », qui n’en révèle pas moins son désir de la mort lorsque " tu " flirte avec " elle ». En revanche, ce désir mystérieux est décrit par la bouche de " tu » et non pas de " elle " même, à travers les histoires des jeunes filles se jetant dans l'eau. Pour ainsi dire, la perception visuelle que " elle » a de l'eau passe par l'autre qui explique oralement ce que " elle " ressent via «tu ». Les histoires des filles, comme une preuve, confirment l'identique perception des femmes à l'égard de l'eau: c'est la mort. Il apparaît que cette perception incarne une psychologie du féminin à travers les sujets percevant une palette de " elle(s) » dans un temps extensible vers les époques lointaines: le temps présent (le partenaire du dialogue " elle ») — les années de la révolution culturelle (la jeune fille noyée) - le temps plus lointain et obscur (la femme fantôme) - ou encore une époque révolue, autrefois (la jeune paysanne). Cette sensation de la mort se transmet d'une « elle ", à l'autre car les raisons de se noyer pour les filles sont analogues: elles éprouvent le même désespoir d'amour, et elles ne peuvent trouver nulle part dans ce monde le vrai amour dont elles rêvent: "Les hommes veulent une femme pour leur propre plaisir, les maris une épouse pour s'occuper de leur maison et faire la

29. Ibid., p. 82-85. 
cuisine, et les vieux une bru pour assurer leur descendance. Aucun ne recherche l'amour ${ }^{30}$. " L'eau reflète le désespoir des jeunes filles et accomplit virtuellement leur désir de mort, comme l'intermédiaire qui déploie la perception de "elle(s) ». Aussi, grâce au même pronom personnel " elle », l'expérience de la noyade fait superposer les images des filles: la dialogueuse " elle ", la jeune fille noyée, la femme fantôme et la jeune paysanne.

De la perception individuelle à la psychologie collective, dans cette transmission, ce qui est exposé n'est pas seulement la perspective personnelle mais aussi la couche profonde de la culture psychologique chinoise voire même universelle.

Dans le chapitre suivant de " tu/elle " (ch.11), " elle " imagine de se noyer, et d'expérimenter la mort dans tous ses détails. Cette imagination exprime sans doute l'expérience des filles noyées qui n'ont pas eu l'occasion de parler:

Elle dit qu'elle aurait dû remonter le fleuve en suivant la rive. Quand elle aurait trouvé une plage, elle serait descendue sur la berge. Bien sûr, personne ne devrait la voir et personne ne le saurait. Elle serait entrée dans l'eau noire en pleine nuit, sans même quitter ses chaussures. Elle ne voulait pas laisser de traces. Elle aurait donc avancé, chaussures aux pieds. Pas à pas, elle serait entrée, et quand elle aurait eu de l'eau jusqu'à la taille, avant même qu'il ne lui arrive à hauteur de poitrine et l'empêche de respirer, le courant serait devenu impétueux et d'un coup l'aurait entraînée et roulée au milieu du fleuve. Elle ne parviendrait plus à remonter à la surface, mais, malgré elle, elle se débattrait. Ce désir instinctif de survie ne servirait à rien. Au plus, elle battrait faiblement des pieds et des mains. Tout irait très vite, tout serait fini sans avoir eu le temps de souffrir. Elle ne pourrait pas crier. Il n'y aurait plus le moindre espoir, mais même si elle criait, l'eau l'étoufferait aussitôt. [...] Il faudra que la mort soit pure, elle aussi ${ }^{31}$.

L'imagination de "la mort pure ", presque poétique, évoque pourtant immédiatement le sentiment de dégoût lorsque " elle " pense le corps mort: «Si elle pouvait mourir dans la pureté, ce serait bien, mais si le corps gonflé d'eau échouait en aval sur une anse de sable, il serait séché par le soleil, commencerait à pourrir et serait

30. Ibid., p. 85

31. Ibid., p. 97-98. 
la proie d'une nuée de mouches ${ }^{32}$. " Dans ce dégoût pour le corpschair, il ne s'agit pourtant pas de ce que " elle " éprouve elle-même pour son corps - «elle " est déjà morte dans son imagination; il s'agit de la répulsion sous l'œil invisible: le regard qu'autrui (dans son imagination, il s'agit notamment de son amoureux infidèle) jette sur son corps exhibé. Le dégoût a un double sens: le corps pourri est dégoût sous le regard d'autrui, et le regard d'autrui sur son corps provoque en " elle » la nausée.

Ainsi, le dégoût pour son propre corps explique la sensation de " elle » à l'égard du désir charnel que l'homme a pour elle. Cette sensation de la nausée apparaît très souvent chez Gao, notamment dans la relation entre l'homme et la femme: comme sentiment originel chez les femmes, mais survenant aussi paradoxalement chez les hommes. Rappelons ici, dans le roman classique Le Rêve dans le pavillon rouge que Gao apprécie beaucoup, la fameuse déclaration du protagoniste Baoyu: " Les filles, leurs os et leurs chairs sont faits d'eau; les garçons, leurs os et leurs chair sont faits de fange. Dès que je vois une fille, je me sens pur et alerte, mais, quand j'aperçois un garçon, je me sens empesté d'ordure et de puanteur ${ }^{33}$. " En fait, ce qui marque l'écart entre les filles raffinées et pures et les hommes grossiers et obscènes, c'est tout d'abord le désir charnel. Dans $L a$ Montagne de l'âme, le désir de « tu ", très lié au crime, s'exprime souvent par la perception de l'eau.

Quand " elle " est entraînée par le sentiment féminin de souffrance, et plonge par la similarité virtuelle dans la tragédie de l'amour qui se répète chez les filles de différentes époques, " tu " s'insère lui-même, visiblement, dans les histoires de sa propre narration, accomplit la perception des femmes mais aussi la sienne propre. Si la perception de l'eau révèle chez les filles le désir de la mort et la rancœur contre les hommes (égoïsme, violence et trahison), cette même perception fait que « tu » éprouve d'emblée du désir pour les femmes. Dans le chapitre suivant (ch. 13), lorsque « tu " raconte le conte des séduisantes femmes au camélia — dont une que « tu » rencontre

32. Ibid., p. 98.

33. CAO, X. Q., Le Rêve dans le pavillon rouge, traduit par Li Tche-Houa et Jacqueline Alézaïs, Paris, Gallimard, 1981, p. 46. 
aussi sur un sentier dans les montagnes -, il introduit bizarrement l'histoire de trois jeunes enlevant une femme au camélia: deux d'entre eux la violent, le troisième la laisse partir par pitié. " Tu » vit une expérience analogue à celle de ce dernier: il n'ose pas offenser la femme au camélia lorsqu'il la rencontre dans la montagne:

Tu dis que tu t'es levé pour partir, mais que tu n'as pu t'empêcher de te retourner pour lui jeter un regard et que tu as vu alors ses deux joues et une fleur rouge de camélia piquée sur sa tempe. La pointe de ses sourcils et le coin de ses lèvres brillaient comme des éclairs, illuminant soudain le vallon sombre. Ton cœur s'est enflammé. Tu as tout de suite compris que tu avais rencontré une femme au camélia. Elle était assise là, bien vivante, et sa poitrine tendait sa chemise de lin bleu clair. [...] Aux pieds, elle portait une paire de chaussures, neuves aussi, de toile bleue à fleurs. Elle se détachait comme un papier découpé sur une fenêtre.

Approche-toi! Elle te fait signe.

Assise sur une pierre, elle ôte d'une main ses chaussures à talon haut et, de son pied nu, tâte les galets avec précaution. Ses orteils blancs ondulent dans l'eau pure, comme des vers charnus. Tu ne comprends pas comment les choses ont commencé. Tu renverses soudain sa tête sur les verts joncs sauvages du bord de l'eau. Elle redresse la taille. De tes doigts, tu recherches l'attache de son soutien-gorge et tu libères ses seins ronds, d'un blanc diaphane sous la lumière du soleil de midi ${ }^{34}$.

Toujours grâce à ce pronom anonyme "elle ", l'image de la femme au camélia et celle de "elle " se superposent. Mais qui fait signe à « tu » ? La femme au camélia ou " elle », ou bien est-ce seulement l'imagination de «tu »? De même, qui est le sujet percevant? " Tu », ou le troisième jeune homme? La scène où ils font l'amour dans laquelle «tu » ne sait pas comment commencer semble tout d'abord la revendication du désir de « tu » aussi bien que de celui du troisième jeune homme dont le désir ne vise pas la femme au camélia mais se transfère vers " elle ». Pourtant, au moment où « tu » accomplit son désir, il voit un oiseau lui rappelant la fille de seize ans morte d'un crime prémédité que « tu » aurait dû empêcher:

Tu vois saillir la pointe rouge de ses seins ronds, d'un blanc diaphane sous la lumière du soleil de midi. Elle pousse un petit cri et ses deux pieds glissent dans l'eau. Un oiseau noir aux pattes blanches, tu sais

34. GAO, X. J., La Montagne de l'âme, op. cit., p. 115. 
que cet oiseau s'appelle la pie-grièche, se pose sur un rocher brunâtre, rond comme un sein au beau milieu du ruisseau. [...] La piegrièche regarde à droite et à gauche, lève la queue, dresse et abaisse son bec rouge cire. À peine t'approches-tu qu'elle s'envole au ras de l'eau; elle va se poser non loin du rocher, continue à s'agiter. Elle te laisse approcher, puis s'envole, puis t'attend en poussant des petits pépiements. Cet esprit malin de couleur noire, c'est elle.

Qui?

Son âme.

Et qui est-elle?

Tu dis qu'elle est morte déjà. Ces bâtards l'ont emmenée pendant la nuit pour se baigner au bord du fleuve. Quand ils sont revenus, ils ont dit qu'ils ne se sont aperçus de sa disparition qu'une fois sur la rive. Des mensonges, bien sûr, mais c'est ce qu'ils ont dit ${ }^{35}$. [...]

En fait, dans les histoires précédentes, apparaissait déjà un oiseau qui s’appelle " tête-bleue » et qui vit au bord de l'eau où les femmes se noient:

Mais, à cet endroit, vit réellement un oiseau aquatique que les hommes du pays appellent tête-bleue et dont les gens cultivés disent qu'il s'agit de l’Oiseau bleu mentionné dans la poésie des Tang. Ce sont les paysans qui le nomment tête-bleue, en raison de ses longues plumes bleues. Tu as déjà vu cet oiseau, bien sûr, petit, un corps bleu foncé avec sur la tête deux huppes émeraude, très habile et plein d'agilité, il a belle allure ${ }^{36}[\ldots]$

Ce que « tu » éprouve pour le tête-bleue comme pour la pie-grièche correspond aux sentiments qu'il ressent envers les femmes les plus raffinées et les plus sensibles, qui finissent toujours par connaître une vie malheureuse. Ainsi, l'oiseau, en tant que symbole des femmes mais aussi concrétisation de la perception que « tu » entretient à propos d'elles, révèle d'une manière très détournée ce désir qui en « tu » accompagne encore le sentiment coupable. Or, le remords de " tu » à propos de la fille morte ne tient pas seulement au fait qu'il n'a pas pu empêcher le crime; la source de ce remords est plutôt à chercher dans le désir qu'il a eu d'elle: c'est ce désir charnel qui le fait s’identifier aux jeunes qui violent et tuent la fille. Le dévoilement des désirs de « tu » aura lieu - mais plus loin, dans le chapitre 32.

35. Ibid., p. 115-116.

36. Ibid., p. 85 . 
Rappelons que les chapitres 31 et 32 sont la seule exception à l'ordre systématiquement alterné. Les récits de "tu/elle " apparaissent deux fois successivement: il semble que « tu " et " elle " se mettent en face à face avec eux-mêmes; en d'autres termes, c'est une introspection pour extérioriser. Les récits dans le chapitre 31 sont le miroir de ceux du chapitre 32, comme " elle " est le miroir de " tu ", et réciproquement:

Raconte-moi si tu l'as vue, si tu as vu cette fille.

Quelle fille?

La fille qui s'est noyée.

La jeune instruite installée à la campagne? La jeune fille qui s'est suicidée en jetant dans le fleuve?

Non.

Laquelle alors?

Celle que vous avez attirée en lui disant que vous alliez vous baigner de nuit et qu'ensuite vous avez violée!

Tu dis que tu n'y étais pas.

Elle dit qu'elle est sûre que tu y étais.

Tu dis que tu peux le jurer!

Eh bien, tu l'as certainement touchée.

Quand?

Sous le pont, dans le noir, toi aussi tu l'as touchée, vous êtes tous mauvais, les garçons!

Tu dis qu’à l'époque tu étais encore petit, tu n’aurais pas osé.

Au moins l'as-tu regardée.

Bien sûr que tu l'as regardée, elle n’était pas d'une beauté ordinaire, elle était vraiment attirante.

Tu ne l'as pas regardée de manière anodine, tu as regardé son corps.

Tu dis que tu y as seulement songé.

C'est faux, tu l'as certainement fait ${ }^{37}$.

[...]

C'est une auto-interrogation rigoureuse dévoilant le désir dissimulé que le moi ne veut plus mentionner. La poursuite de la perception à travers l'histoire de " tu/elle " n'est pas seulement révélatrice du désir, mais aussi de la vérité. De quoi s'agit-il? De la vérité, dans le processus de la perception? D’une sensation véritable? D’un souvenir fondé sur le réel? Comment faire émerger la vérité dans la perception?

37. Ibid., p. 258. 
Dans La Montagne de l'âme, la vérité ne se présente jamais comme substrat au-dessous du phénomène, elle apparaît toujours en accompagnement de son ombre « Lingshan ».

\section{L’ÉTAT DE LA VÉRITÉ}

\section{Lingshan et la vérité}

\section{Le jeu de mots}

Lingshan (la montagne Ling 灵山), traduit en français « la montagne de l'âme ", est un endroit dont " tu » a entendu par hasard quelqu'un parler dans un train; " tu " n'en avait jamais entendu rien dire auparavant, et il était impossible qu'il trouvât cet endroit dans les guides touristiques. C'est une montagne de la forêt vierge à la source de la You: cette dernière se trouve dans un petit bourg, Wuyi, d'où l'on peut remonter la You en barque. Il y a un jeu de mots sur les noms dès ce premier chapitre du roman. Le caractère You (尤), le nom de la source, signifie " distinct » ou « différent » dans les textes antiques; il se compose souvent d'un autre caractère " chose 物》 (Youwu 尤物 ${ }^{38}$ ), signifiant « la femme qui a une beauté ravissante ", dans la littérature classique aussi bien que moderne. La source de la You révèle d'emblée son attribut féminin: il y a sans doute là une métaphore de la féminité de l'eau issue de l'inconscient culturel.

Mais pour trouver la source de la You et la montagne Ling, il faut aller dans le bourg Wuyi. Le nom Wuyi (乌伊), implique tout d'abord la signification « il n'y a pas ", marquée notamment par le caractère $W u$ (乌): déjà depuis plus de deux mille ans, le personnage de Monsieur Wuyou (乌有) chez Sima Xiangru ${ }^{39}$ est le synonyme de ce qui n'existe pas. Par exemple, lorsque le terme d'utopie est entré en Chine au siècle dernier, il a été traduit par Wutuoban (乌 托邦). Ainsi, le nom de Wuyi annonce un endroit qui n'existe pas,

38. Par exemple, dans 《左传·昭公二十八年》 (Zuozhuang) : 《夫有尤物》 (《I y a une fille distincte »), Changsha, Yuelu Chubanshe (岳麓出版社), 1995, p. 356.

39. Sima Xiangru (司馬相如, 179 - 117 av.J.C.) est un poète chinois, principal représentant du genre fu (赋). Voir 《司马相如集校注.子虚赋》(Sima Xiangru Jijiaozhu), Shanghai, Shanghai Guji Chubanshe (上海古籍出版社), 1993, p. 1-30. 
ou, du moins, qui n'existe pas dans le monde réel. Dans le premier chapitre, lorsque « tu » cherche dans une gare routière l'itinéraire menant au Wuyi, il trouve tout d'un coup le nom Wuyi écrit au bout d'une longue liste de noms de gares qui apparaissent de plus en plus jolis et semblent s'éloigner du monde réel l'un plus que l'autre: « Le Village des Zhang, la Boutique de Sable, l'Usine de Ciment, le Vieux Four, Cheval d'or, Bonne Année, Inondation, la Baie du dragon, le Bassin des Fleurs de Pêcher ${ }^{40} \ldots$...

"Le bassin des Fleurs de Pêcher ", nom de la dernière gare avant celle de Wuyi, évoque obligatoirement la fameuse fable de Tao Yuanming: La Source des Fleurs de Pêcher. Cette fable symbolique, qui décrit un pays idéal et parfait situé au-delà du monde, a grandement et constamment influencé la littérature et la pensée chinoises. Gao en choisissant ce nom, nous indique malicieusement que le chemin pour entrer dans la montagne Ling, comme dans la fable celui qui mène à la source des Fleurs de Pêcher, est a priori introuvable, quelle que soit la volonté d'y parvenir. C'est au hasard d'un détour que le pêcheur, héros de la fable, tombe sur la source des Fleurs de Pêcher, après avoir perdu le chemin qui devait selon lui en permettre l'accès. Ce clin d'œil à l'allégorie donne à penser quant à la suite du voyage de " tu " pour Lingshan (la montagne Ling): pourra-t-il trouver un tel endroit mystérieux dont il a ouï dire, en le cherchant à tout prix? D'ailleurs, à propos, de quoi s'agit-il, que signifie la montagne Ling?

C'est à la fois le nom de la montagne et le titre du roman. Lingshan est une référence culturelle, qui évoque le souvenir d'un mythe lointain:

Tu n'ignores pas que dans les innombrables livres et textes historiques anciens, depuis le Classique des mers et des montagnes, ouvrage de divination et de magie antique, jusqu'au vieux traité de géographie intitulé Annotations au Classique des rivières, le site de Lingshan est bien mentionné. Le bouddha y a même donné l'éveil au vénérable Mahakasyapa ${ }^{41}$.

40. GAO, X. J., La Montagne de l'âme, op. cit., p. 15.

41. Ibid. 
«Ling " recèle de nombreuses significations: l'âme, l'être spirituel, le sorcier, etc., comme substantif; merveilleux, éveillé, comme adjectif. Si l'on remonte à l'origine de ce caractère, Ling (灵), qui est écrit dans la langue traditionnelle 靈, est composé de trois parties: le sorcier (巫) au-dessous, les trois bouches (口口口) au milieu et la pluie (雨) au-dessus. Il signifie au sens primitif la cérémonie de la divination ou de la prière (par exemple, prier pour la pluie). Cela correspond à une mentalité primitive de $\mathrm{Wu}$ (divination, 巫), l'origine de la culture de Chu née dans le bassin du fleuve Bleu, dont les confucianistes, originaires du bassin du fleuve Jaune, se méfient ${ }^{42}$.

Ainsi, comme Gao adhère à la culture du bassin du fleuve Bleu qui est en marge de celle du confucianisme en tant qu'idéologie dominante de la société chinoise depuis deux cents ans avant notre ère, dans La Montagne de l'âme, la signification de Ling s'attache étroitement à la culture Wu. En effet, Ling et Wu sont souvent identifiées dans les textes antiques: dans Classique des montagnes et des mers que Gao mentionne pour marquer l'origine de la montagne Ling, Lingshan n'est qu'un autre nom de Wushang ${ }^{43}$ (la montagne de $\mathrm{Wu}$ 巫山); et c'est ainsi que dans certains vers de Quyuan (屈原), l'auteur de Chuci (楚辞), les plus fameux poèmes issus de la culture de Chu, Ling et $W u$ sont interchangeables ${ }^{44}$. À n'en pas douter, Gao

42. 《子不语怪力乱神》, dans 《论语·述而》. Voici la traduction d'Anne Cheng dans Entretiens de Confucius: "Le Maître ne parlait jamais de l'étrange ni des esprits, de la force brute ni des actes contre nature » (Paris, Seuil, 1981, p. 65) ou celle d'André Levy dans Les Entretiens de Confucius et de ses disciples: "Le maître ne parlait pas de prodiges, de violences, de désordres ou de génies » (Paris, GF-Flammarion, 1994, p. 61.)

43. 《山海经》 (Classique des montagnes et des mers) : 《大荒之中有山, 名曰丰沮玉 门, 日月所入。有灵山, 巫咸、巫即、巫盼、巫彭... »Yuan Ke (袁珂) explique en dessous: 《La montagne de Ling est sans doute la montagne de Wu » (《灵山, 疑即巫 山 »), voir «山海经校注 » (Les notes du Classique des montagnes et des mers), Shanghai, Shanghai guji chubanshe, 1980, p. 396.

44. Wang Guowei (王国维) a cité Les Chants de Chu de Qu Yuan pour montrer que Wu et Ling signifient le même sens dans la culture Chu. Voir dans un poème Donghuang Taiyi des Chants de Chu ( «楚辞・ 九歌・东皇太一») : «les déesses (Ling) dansent en revêtant les belles robes 》 (灵偃塞兮皎服), Wangyi (王逸) note: “Ce (les déesses, Ling) sont des sorcières (Wu) » (巫也) ; aussi dans un autre poème Yunzhongjun (《( «楚辞・九歌・ 云中君 》) des Chants de Chu: 《Le dieu de nuage demeure [sur la nuage] (灵连蜷兮既 留), Wangyi note encore: 《les gens du Chu nomment Wu en Ling》(楚人名巫为灵子). Nous référons 《宋元戏曲史》 (L'Histoire du Xiqu des Songs et des Yuans) de Wang Guowei, Beijing, Dongfang chubanshe (东方出版社), 1996, p. 6. 
joue sur ces mots pour marquer son attachement à la culture de $\mathrm{Chu} / \mathrm{Wu}$ et son éloignement de la culture confucianiste.

\section{Lingshan et la vérité: se reflètent et s'étendent}

Dès le premier chapitre de La Montagne de l'âme, en impliquant les signifiants et les noms allusifs des lieux, l'auteur révèle que le voyage de " tu/elle " se déroule dans un monde imaginaire plutôt que réel; dans un monde intérieur et spirituel plutôt qu'extérieur et concret. Signifie-t-il ainsi que Lingshan n'existe que dans les mythes lointains, et qu'il ne peut qu'être entrevu en remontant dans la culture perdue de Chu?

En effet, on ne peut pas comprendre Lingshan sans entrer dans un ensemble métaphorique, et on ne peut pas le comprendre non plus seulement dans cet ensemble métaphorique. Lingshan existe en tant que telle et, simultanément, pas seulement en tant que telle. Lingshan est tout d'abord un rêve idéal que l'on cherche audelà du monde, quelque part dans l'autre pôle de la vérité. Gao met ainsi dans une relation de tension Lingshan et la vérité, et en fait la quintessence de la construction de son roman, notamment à travers le jeu de l'alternance des chapitres « tu/elle " et « je ». La polarité Lingshan/vérité se reflète à travers celle de " tu " et de " je ». De même que " tu " est l'ombre de " je ", Lingshan est l'ombre de la vérité; et réciproquement, Lingshan et la vérité prévoient respectivement le destin de " tu » et de « je ": "Quant à toi, tu es à la recherche du chemin qui mène à Lingshan, moi, en me promenant le long du Yanzi, je recherche la véritée ${ }^{45}$."

En effet, Lingshan et la vérité ne se reflètent pas seulement l'un l'autre, mais encore s'étendent l'un vers l'autre. Tout comme les récits de «tu " et de "je » s'unissent par fusion à la fin du roman, la frontière entre Lingshan et la vérité s'estompe: dans le chapitre 51, « je » cherche l'origine de la montagne Wu (巫山) dans la quête de quelques fragments d'annales, pendant que "tu " et «elle» cherchent en vain Lingshan dans le monde spirituel. Il semble que « je » essaie de prouver l'existence de Lingshan par les traces

45. GAO, X. J., La Montagne de l'âme, op. cit., p. 25. 
historiques de la montagne $\mathrm{Wu}$ - le synonyme de la montagne Ling, dans les documents notés à travers toutes les dynasties chinoises:

«À l'époque des Tang Yao, le mont Wu a tiré son nom de Wu Xian, Wu Xian était le médecin au vaste savoir-faire de l'empereur Yao, né dans une famille de ministre de haut rang, il est mort comme grand sage, son fief était la montagne à qui il a donné son nom (cf. Guo Pu: Élégies des monts Wuxian)

"À l'époque de Yu Shun, le Classique de l'empereur Shun indique: le mont Wu appartient aux régions de Jing et de Liang.

"Sous le Xia, l'empereur Yu divise l'empire en neuf régions et le mont Wu se trouve toujours dans la région de Jing et Liang. " "Sous les Shang, dans l'Éloge des Shang, Neuf possessions, neuf encerclements, il est noté: Les régions auxquelles le mont Wu appartient ne différent pas de l'époque des Xia. »

[...]

"Sous les Qing, en l'an 9 du règne de Kangxi, Dachang est supprimé et intégré dans le district de Wushan ${ }^{46}$... "

Rappelons que la première rencontre entre « je ", «tu » et « elle » ayant lieu au chapitre 52. Cette exploration littéraire fait fusionner l'image de la montagne Wu et l'image de la montagne Ling, et aussi la légende historique et la vérité dans des documents où les indices sont de plus en plus flous. Ainsi dans le dernier chapitre, lorsque " je " ne sait plus où il se trouve, il comprend que Lingshan est comme la vérité, et comme une perception en lui.

\section{La vérité - zhenshi}

Mais qu'est-ce que la vérité dans La Montagne de l'âme? Il faut préciser tout abord que Gao n'utilise dans le texte chinois qu'un vocable, zhenshi (真实), pour désigner ce que "je » recherche; dans la traduction française, en revanche, zhenshi est rendu par "vérité », " réel » ou "vrai » selon le contexte. C'est que zhenshi, tel que Gao l'utilise, relève plutôt de la pensée traditionnelle que de la vérité au sens moderne, importé d'Occident. Notamment, le zhen (真) chinois est presque impossible à traduire en respectant son sens propre, quel que soit le nombre des mots et expressions disponibles pour exprimer la « vérité » et quelle qu'ait été l'évolution de ce qu’on

46. Ibid., p. 418-420. 
entend par ce concept dans la philosophie occidentale, de la Grèce antique à la pensée contemporaine.

Léon Vandermeersch appelle la «logique " qui fonde la pensée chinoise une morpho-logique - il se réfère à la structure de l'idéogramme: "La pensée spéculative n’a cessé de s'exercer, pour la rectifier et l'affiner suivant sa morpho-logique, sur l'intuition brute du monde fixée dans la façon idéographique de dire les choses, encore très immature et se retenant mal de glisser à la divagation $^{47}$. " Qu'en sera-t-il dans le cas qui nous occupe? À remonter à l'origine de l'idéographie de ces deux caractères zhen (真) et shi (实), on va pouvoir mesurer combien le zhenshi se trouve dès le début à l'antipode de la vérité en Occident...

Dans le style du bronze (jinwen, 金文) qui apparaît à la fin de dynastie Shang (environs dix siècles avant notre ère), zhen s'écrit ${ }^{48}$ 魏, composé de $\boldsymbol{\Gamma}$ (la cuillère) et de 暴 (une sorte du vase tripode muni de deux anses qui servait pour les offrandes, ou comme marmite pour la cuisine): selon l'image du caractère, zhen implique une action de goûter ce qui est cuisiné dans la " marmite " avec une cuillère. Malgré la perte de cette signification originelle, on peut donc considérer que le zhen provient d'un sens concret et non pas d'une idée abstraite.

Quant au caractère shi (实), il est encore plus "matériel » que celui de zhen, eu égard à sa forme originelle 䨳 dans le style du bronze; elle est déjà similaire à la forme classique 實, composée de (le toit, signifie la maison), Ila caisse) et signifie l'argent): bref, la fortune déposée dans la caisse à la maison. De là le sens premier de shi: "riche ", puis son sens figuré: "plein » (满) à l'opposé de « vide (空) » et partant de là : « tout que ce qui peut être touché réellement, ou existe vraiment ", à l'opposé de "rien (虚)».

La forme de zhen a beaucoup évolué: à la période des Printemps et des Automnes, zhen s'écrit 萛, idéogramme déjà très proche de la

47. VANDERMEERSCH, L., Les Deux Raisons de la pensée chinoise. Divination et idéographie, Paris, Gallimard, 2013, p. 118.

48. Rond, G., ZhANG, Z. L., et MA, G. Q., «金文编 》 (Jinwen bian), 容庚编著, 张振林, 马 国权补编, Beijing, Zhonghua shuju, 1985, p. 575. 
forme moderne. Dans le premier dictionnaire de caractères chinois, Shuo Wen Jie Zi (“说文解字 ») qui date du début du II ${ }^{\mathrm{e}}$ siècle, l'auteur Xu Shen explique que zhen, composé en partie de l'œil (目) et en partie de l'oiseau noir $\left(L^{49}\right)$, signifie "le céleste qui se transforme (en oiseau?) afin de monter au ciel $^{50} "$. De ce point de vue, zhen ne se ramène à aucun des sens de la vérité dans la terminologie occidentale.

L'explication de $\mathrm{Xu}$ Shen est sans doute inspirée de la culture de Chu, comme le furent Quyuan et Zhuangzi: le terme zhenren (真人) qui apparaît plusieurs fois chez les deux auteurs est, notamment chez Quyuan, synonyme de " céleste $^{51}$ " Chez Zhuangzi, alors même que la religion taoïste lui donne aussi ce sens de "céleste", il représente plutôt une figure concrète du Dao: "Il y aura des connaissances authentiques lorsqu'il y a un homme authentique ${ }^{52}$ (zhenren).»

Qu'est-ce qu'un homme authentique? L'homme authentique de l'antiquité ne s'opposait pas à la minorité, ne faisait pas étalage de son succès et n'aguichait pas les hommes. Un tel homme ne regrettait pas ses erreurs et ne tirait pas fierté de ses bonnes actions. [...] l'homme authentique ne se connaissait ni à se faire plaisir de la vie, ni à détester l'horreur de la mort; il ne se réjouissait point en entrant et ne redoutait point en sortant; il s'en allait tout naturellement comme il était venu, sans plus. Il n’oubliait pas où il avait commencé et ne cherchait pas où il se terminerait, il se contentait de ce qui lui était donné et considérait que toute perte n'était qu'un retour ${ }^{33}$.

49. Voir Xu, S., Shuo Wen Jie Zi ( 《说文解字 ») : 'L'est expliqué aussi par Xu comme le pictogramme de l'oiseau noir ( ᄂ, 玄鳥也。齊魯謂之し。取其鳴自呼。象形。凡 之屬借从し), Beijing, Zhonghua shuju, 2002, p. 779.

50. Ibid., p. 537, en chinois : 眞, 僊人變形而登天也

51. Les Chants de Chu ( 《王注楚辞・九思・哀岁》) 《vole avec Zhenren》(随真人兮 朝翔), Wangyi (王逸) note: 《zhen, soit disant le céleste» (真, 仙人也), Shanghai, Zhonghua tushuguan (中华图书馆), 1917 (民国六年), livre 4, vol.17, p. 10.

52. ZHuANGzi, Zhuangzi Jishi ( 《庄子集释・大宗师》), Beijing, Zhonghua shuju, 1961， p. 226 : 《且有真人后有真知》

53. Ibid., p. 228. Nous nous inspirons de la traduction de A. C. Graham: Chuang-Tzu, the Inner Chapters (London, Boston, Allen \& Unwin, 1981), de Jean Levi : Les E Fuvres de maître Tchouang (Paris, L'Encyclopédie des nuisances, 2006) et de Liou Kia-Hwat: L'Euvre complète de Tchouang-tseu (Paris, Gallimard, 1969). 
L'homme authentique est celui qui « n'endommageait pas le Dao par son esprit et qui ne prétendait pas aider le ciel ${ }^{54}$ ». En d'autres termes, l'homme authentique ne recourt ni à l'action ni à l'idée et, en ignorant à la fois le " moi " et la "vérité ", il réalise pourtant exactement le zhen/Dao.

Ces notions de zhen et de zhenren ne se déploient, on le voit, ni sous l'angle de l'objectivisme ni sous celui du subjectivisme: or, la notion occidentale de vérité, depuis l'idéalisme platonicien (chacun a en tête l'allégorie de la caverne), est jusqu'à nos jours prétexte à une controverse infinie sur sa nature: subjective ou objective ${ }^{55}$ ? Le zhen correspond au zhenren: comme le zhen ne représente pas une vérité objective, le zhenren n'implique pas non plus un « moi » subjectif. Le zhen, chez Zhuangzi, se réfère à la fois à la nature propre du moi (本真) et à un moi qui s'oublie, c'est-à-dire un non-moi.

Gao utilise zhenshi (真实) au lieu de zhenxiang (真相) ou zhenli (真理) pour dire «la vérité »: cela implique d'emblée que son interprétation de la vérité combine les deux sens originaux de ces deux caractères zhen et shi. L'un adhère à la " vérité » telle qu'elle est énoncée par Zhuangzi, il s'agit d'une perception du " moi », et non d'une quelconque idée "moderne » de la vérité mise en circulation depuis le début du $\mathrm{xx}^{\mathrm{e}}$ siècle sur la base de la dialectique objet/sujet. L'autre, shi, met l'accent sur le réel sans dépendre de la perspective du moi.

Ainsi, pour Gao, la vérité est à « l'intersection du sujet et de l'objet ${ }^{56} »$, autrement dit, elle n'est ni purement subjective, ni purement

\section{Ibid.}

55. C'est pourquoi Deleuze cherche une «nouvelle critique » chez Proust qui, selon lui, échappe au « piège de l'objet » et aux « rets de la subjectivité » et aidera la philosophie à sortir son paradoxe à l'égard de la vérité. II s'agit d'un monde des signes qui déchiffre la vérité et fait émerger les essences « au-delà des objets désignés, au-delà des vérités intelligibles et formulées; mais aussi au-delà des chaînes d'association subjectives et des résurrections par ressemblance ou contiguïté » (Proust et les signes, Paris, Puf, 1964, (Quadrige, 2007), p. 50). D'après Deleuze, Proust oppose le monde des signes et des symptômes au monde des attributs, le monde du pathos au monde du Logos, le monde des hiéroglyphes et des idéogrammes au monde de l'expression analytique, de l'écriture phonétique et de la pensée rationnelle: «Ce qui est récusé constamment, ce sont les grands thèmes hérités des Grecs : le philos, la sophia, le dialogue, le logos, la phoné. » (Proust et les signes, p. 131).

56. GAO, X. J., «没有主义》, Ne pas avoir de -isme, op, cit., 2001, p. 84. 
objective. Si l'école moderne du début des années 1980, dont Gao fut le pionnier, marqua son opposition aux théories réalistes qui voient dans la vérité une notion objective, dans La Montagne de l'âme, "je " doute aussi de l'idée de la vérité dans la mesure où elle se situerait seulement au niveau de l'esprit:

Je veux quitter le monde littéraire en pleine effervescence et m'enfuir de ma chambre toujours remplie de fumée de tabac. Les livres qui s'y entassent m'oppressent, au point de m'empêcher de respirer. Ils exposent toutes sortes de vérités, depuis la vérité historique jusqu'à la vérité du comportement humain, et je ne sais plus quelle utilité elles ont. Pourtant, elles m'entravent et je me débats dans leurs filets, vivant comme un insecte pris au piège d'une toile d'araignée ${ }^{57}$.

\section{Qui cherche et éprouve la vérité?}

Mais qui cherche la vérité et qui éprouve la vérité? C'est une question qui ne se pose pas chez Zhuangzi, mais qui est incontournable chez Gao. Car le moi taoïste et le moi chez Gao ne sont pas les mêmes, quoi qu'il en soit: une fois la conscience du sujet mise en éveil, le moi émerge malgré l'avantage que présente l'hétérogénéité de la pensée chinoise par rapport à la pensée occidentale. D’où cette présence chez Gao de l'ombre que les deux moi projettent sur la vérité, une ombre qui fait apparaître d'emblée son idée de la vérité comme un paradoxe:

Cette vérité (zhenshi) ne peut être bien expliquée et ne peut être définie mais elle existe vraiment [...]. Elle n'est pas la même que le réel dans la vie quotidienne sur lequel on a l'expérience propre ${ }^{58}[\ldots]$

Alors, comment atteindre une telle vérité si elle ne coïncide plus avec une perspective intellectualiste? Comment éprouver les choses qui " existent vraiment »? S'agit-il désormais, d'une perception personnelle? Mais n'y a-t-il pas le risque de retomber dans le piège du solipsisme où la perception du moi s'étendrait jusqu'à recouvrir la "vérité "? Pour parer à ce risque, lorsque les questions convergent à nouveau sur qui doit chercher et comment chercher la vérité, Gao essaie de répondre autrement.

57. GAO, X. J., La Montagne de l'âme, op. cit., p. 26.

58. Ibid. 
Dans La Montagne de l'âme, la première fois que " je " éprouve la sensation de la vérité (zhenshi), c'est quand il s'enfuit de toutes les discussions étouffantes de Beijing, et se trouve à mi-chemin entre les hauts plateaux tibétains et le bassin du Sichuan, au pays de l'ethnie Qiang, dans la partie médiane des monts Qionglai:

Assis devant le feu, il boit de l'alcool mais, avant d'y goûter, il trempe un doigt dans son bol et l'agite au-dessus des braises qui se mettent à siffler en crachant une fumée bleue. À cet instant, je réalise que j'existe vraiment ${ }^{59}$ (我也才觉得我是真实的).

Ce n’est pas « je » qui éprouve la perception de la vérité bien que « je » la cherche - autrement dit, dans cette sensation de la vérité, « je » est passif. De même pour la question de qui cherche la vérité: le sujet qui cherche et le sujet qui éprouve la vérité ne sont pas identiques. Comme le vrai sujet percevant n'est pas forcément en moi, la vérité que « je » éprouve n’est pas forcément une cohérence entre la pensée et l'objet pensé; il s’agit plutôt d'un état - mais un état où rien n’est "stable ». Cette notion de la vérité est certainement inspirée de la pensée chinoise qui considère tous les phénomènes comme un processus sans fin de changement. Comme le dit Léon Vandermeersch, " dans la pensée chinoise [...] les phénomènes sont représentés d'emblée comme changeants. La permanence de leur substance n'est que relative à leurs transformations, au lieu que, chez Aristote, ce sont les changements qui sont relatifs à la permanence de la substance ${ }^{60} »$.

Dans un dialogue de Gao avec Denis Bourgeois, les deux écrivains, confrontant leurs expériences de l'écriture, en viennent à discuter sur cet état du réel/vérité (zhenshi):

D. B. - Mais bon, recentrons la question sur la pratique de l'écriture: quand tu as vu que toutes les réalités s'évanouissaient devant toi, quand tu as pu constater que tu te retrouvais face à toi-même, devant ta page blanche en train d'écrire, et que même « toi-même » n'est en rien une réalité, un peu comme dans la tradition bouddhiste où l'on parle simplement des agrégats, des choses qui se conglomèrent sans

59. Ibid., p. 23.

60. VANDERMEERSCH, L., Les Deux Raisons de la pensée chinoise. Divination et idéographie, op. cit., p. 116. 
pour autant faire un "soi ", un "sujet ». Cela veut aussi dire que tu n'as plus aucune prise sur le réel, tu es comme suspendu au milieu du vide. Et pourtant, voilà ce qui est étrange entre tout: plus tu es suspendu au-dessus du vide, plus tu vois les repères disparaître les uns après les autres, plus tu es proche, dans ton écriture, de cette rencontre effective avec le réel. Ressens-tu aussi cela? Moi, c'est quand j'ai l'impression d'avoir tout perdu, et d'être derrière la fatigue; c'est quand je ne sais plus ce que je dis que j'écris le mieux...

G. X. - Ce n'est pas tant de la fatigue qu'un profond apaisement intérieur; l'esprit est alors beaucoup plus libre; c'est un bon état pour entrer dans l'écriture: comme après une longue excursion, tu prends une douche, puis retournes devant le bureau, l'esprit est soulagé, prêt à percevoir l'impossible, l'insaisissable qu'est le réel (zhenshi).

Personne n'est fait pour écrire, c'est faux. Si nous écrivons seulement les choses que nous avons vécues, nous n'arrivons à rien. C'est ce réel que nous cherchons à atteindre, et quel est-il? Il n'est pas l'ensemble des souvenirs ou des situations vécues, c'est derrière ce que nous avons déjà vécu. Il y a toujours quelque chose d'inamissible. Nous sommes toujours en quête de ce réel insaisissable, impossible, que nous n'avons pas encore connu ${ }^{61}$.

La vérité (zhenshi) est " impossible » et « insaisissable », elle " n’a pas un caractère d'entité. Mais si on la considère comme ce qui est purement spirituel, elle est pourtant réelle ${ }^{62}$ (实实在在)". Le zhenshi ne peut être atteint ni par la connaissance du sujet - la langue logique par exemple —, ni par la substance de l'objet: il le sera dans un état où le corps se relaxe et l'esprit peut se laisser aller. Un état où la conscience du moi est ignorée mais la perception personnelle accentuée. Autrement dit, le zhenshi est un état perceptible entre le moi et le non-moi.

Voici un paragraphe de "je " dans La Montagne de l'âme qui montre un tel état où la vérité n'est qu'une expérience personnelle:

La vérité n'existe que dans l'expérience et encore seulement dans l'expérience de chacun, et même dans ce cas, dès qu'elle est rapportée, elle devient histoire. Il est impossible de démontrer la vérité des faits et il ne faut pas le faire. Laissons les habiles dialecticiens débattre sur la vérité de la vie. Ce qui est important, c’est la vie elle-

61. GAO, X. J., La Raison d'être de la littérature, suivi de Au plus près du réel, dialogues avec Denis Bourgeois, Gémenos, L'Aube poche, 2001, p. 58-59.

62. GAO, X. J., «没有主义》, Ne pas avoir de-isme, op. cit., p. 84. 
même. Ce qui est réel, c'est que je suis assis à côté de ce feu, dans cette pièce noircie par la fumée de l'huile, que je vois ces flammes, dansant dans ses yeux, ce qui est vrai, c'est moi-même, c'est la sensation fugitive que je viens d'éprouver, impossible à transmettre à autrui. Dehors, le brouillard est tombé, les montagnes sombres se sont estompées, le son de la rivière rapide résonne en toi et ça suffit ${ }^{63}$.

\section{UN AUTRE MODE DU PERCEVOIR: L'« ENTRE » (间)}

\section{Comment percevoir le zhenshi et l'acteur neutre}

Ainsi, le zhenshi chez Gao ne se résume pas à un concept qui puisse être déduit, ni à une entité réelle à laquelle on puisse accéder; il se réfère à un autre mode du percevoir inspiré sans doute de sa propre expérience du théâtre.

En effet, Gao est un dramaturge avant d'être un romancier. Il se familiarise avec le théâtre dès son enfance grâce à sa mère qui était actrice et qui faisait jouer à ses deux fils, avec elle, de petites pièces dans le cadre familial. Cet intérêt pour le théâtre s'est imposé à lui comme l'un des moteurs de sa vie littéraire. Durant ses études de français à Beijing, Gao fut l'un des metteurs en scène de la troupe « La mouette (海鸥剧社)» qu'il avait fondée avec ses amis. Dans un spectacle de soirée de fin d'études, il joue Valère, dans l'Avare de Molière en français, selon sa propre interprétation du rôle, au lieu de se soumettre à l'injonction stanislavskienne qui dominait la critique théâtrale chinoise depuis les années 1940. Le spectacle fut un plein succès et, dès lors, Gao prit la décision de s’imposer comme dramaturge. Ses premières créations littéraires furent ainsi des pièces de théâtre - qu'il brûla pendant la révolution culturelle, vu le risque qu'elles représentaient dans un tel environnement politique.

Bien que le théâtre occidental moderne et contemporain ait eu beaucoup d'influence sur lui, Gao s'est attaché à puiser aux racines de l'opéra (戏剧) chinois traditionnel:

Les deux dramaturges occidentaux qui m'ont inspiré plus, c'est Brecht et Artaud [...] ils admirent tous les théâtres orientaux. [...] Ils me font réfléchir: si les Occidentaux peuvent avoir l'inspiration des

63. GAO, X. J., La Montagne de l'âme, op. cit., p. 30. 
théâtres orientaux lorsqu'ils y jettent seulement un clin d'œil, alors moi, en tant que Chinois, je dois réfléchir quelle semence je pourrai avoir de ces théâtres orientaux traditionnels ${ }^{64}$.

Aussi, cherche-t-il une inspiration féconde pour un " théâtre moderne " en remontant à l'origine de sa culture: il ne procèdera pas autrement pour définir la forme et le rôle d'un roman moderne.

Henry Zhao a montré que les dramaturges chinois des années 1980 avaient pour une bonne part redécouvert l’opéra traditionnel à travers les yeux de leurs homologues occidentaux ${ }^{65}$. Or, selon lui, certaines observations des dramaturges occidentaux à propos de l'opéra chinois relevaient du malentendu, voire même d'interprétations volontairement faussées afin d'avoir une théorie alternative à opposer au système stanislavskien; le paradoxe étant que les dramaturges chinois d'alors adoptaient hâtivement la théorie stanislavskienne pour s'émanciper du théâtre réaliste socialiste ${ }^{66}$. Conscient du processus, Zhao critique notamment Brecht — et son incontournable "distanciation ${ }^{67}$ » - , qui a mal compris selon lui la notion de vérité/réel (zhen) dans le théâtre/opéra chinois traditionnel (xiqu):

Les anciens théoriciens de drame chinois n’ont jamais cherché un xiqu non réaliste. Tang Xianzu, le plus grand des dramaturges classiques chinois, disait: "ceux qui jouent les femmes doivent penser eux-mêmes comme les femmes et ceux qui jouent les hommes doivent toujours essayer de s'identifier aux personnages. " Zang Jinshu, l'auteur d'Anthologie de Yuanqu («元曲选 »), affirme que les ex-

64. “土地、人民、流亡一叶石涛、高行健文学对话》(《Dialogue de la littérature entre Ye shitao et Gao Xingjian »), dans 《高行健一台湾文化之旅》(Gao Xingjian - un voyage de la culture à Taiwan), Taibei, Wenjianhui (文建會出版社), 2001, p. 123-124.

65. ZHAO, H., Towards A Merdern Zen Theatre. Gao Xingjian and Chinese Theatre Experimentalism, London, School of Oriental \& African Studies, 2000, p. 39.

66. Ibid., p. 40

67. Voici l'observation de Brecht: «The [Chinese] performer's self-observation, an artful and artistic act of self-alienation, actor in the performance of self-observation is an art and the art of self- alienation, stopped the spectator from losing himself in the character completely, i. e. to the point of giving up his own identity, and lent splendid remoteness to the event ». (Brecht on Theatre: the Development of an Aesthetic, edited and translated by John Willett, London, Eyre Methuen, 1964, p. 92-93.) 
cellents acteurs se mettent toujours dans l'histoire qu'ils jouent tout en oubliant que ce n'est qu'une fausse histoire ${ }^{68}$.

Ainsi Zhao conclut-il que, dans le xiqu traditionnel, l’idée d'authenticité ou de "jouer le rôle comme s’il [était] vrai » étaient partagées par tous.

Il semble néanmoins que Zhao mélange la ressemblance dans l'art de l'acteur et la pensée de la vérité/réel — zhen — dans le théâtre. D’autant plus que "jouer le rôle comme s’il [était] vrai " n'est qu'une petite partie de l'entraînement de l'acteur; la partie la plus importante que Zhao ne mentionne pas, dont la formule est “jouer dans l'état de stylisation ou dans l'état corporel » (程式化), est en fait la base de la distanciation brechtienne. Par ailleurs, les exemples que Zhao donne ne peuvent pas prouver que la manière de chercher le zhen dans le xiqu traditionnel s'identifie à la représentation du réel dans le théâtre classique occidental.

Les esthétiques du xiqu et du théâtre occidental se différencient par la façon de présenter le réel: xiqu ne cherche pas à imiter le réel mais tente de montrer comment l'on perçoit le réel.

La première preuve que le xiqu traditionnel n'a jamais l'intention de représenter le réel par l'imitation, c’est que "représenter/ représentation ", comme notion, n’existe pas dans la théorie qui est la sienne. C'est ce qui fonde Brecht à parler de distanciation à propos de la "représentation » du xiqu, qui n'installe pas une semblance du réel ou du sentiment pour provoquer l'empathie des spectateurs. Sur la scène du xiqu, il n'y a même pas de décor - à part deux chaises et une table qui supposent une sorte de " temps et espace " sans limite et qui invitent les spectateurs à imaginer ensemble le drame, au lieu de représenter devant eux un réel imité. De même, les stylisations (程式化) du spectacle impliquent un caractère hypothétique - la suppositionalité théâtrale $^{69}$ — qui ne représente pas le réel mais transfère la sensation du réel. En effet, l'esthétique du xiqu

68. Voir 《高行健与中国实验戏剧-建立一种现代禅剧》, Taibei, Erya, 2001, p. 39-40. Towards A Modern Zen Theatre. Gao Xingjian and Chinese Theatre Experimentalism, op. cit., p. 38. Celle-là est la version chinoise de celle-ci, traduite et réécrite par l'auteur lui-même, ainsi les deux versions ne sont pas tout à fait identiques. Notre citation se réfère aux deux versions.

69.En anglais : suppositionality of theater, en chinois : 剧场假定性. 
traditionnel, dans la mesure où elle cherche une similitude spirituelle et non pas formelle (神似形不似), correspond à celle de la peinture et de la poésie traditionnelles. Car la vérité/réel (zhen) est insaisissable; on ne peut, pour le zhen, qu'en exprimer la perception.

De ce point de vue, on comprendra pourquoi Gao ne souscrit pas aux idées de Peter Brook lorsqu'il voit ce dernier étaler du sable sur la scène afin d'avoir une vraie sensation du toucher sur le $\mathrm{sol}^{70}$ : c'est toujours une imitation. Or, «le but du jeu n'est pas d'imiter, l'effort pour "ressembler" occupe seulement les échelons inférieurs de l'expression théâtrale ${ }^{71}$ ».

Pour être clair, Gao comprend vite que les façons d'interpréter la notion de vérité/réel sur la scène dans la tradition de l'Occident et de l'Orient sont opposées:

Tandis que le théâtre de tradition occidentale recherche un sentiment d'authenticité sur la scène, en prenant la voie du vécu psychologique, le théâtre de tradition orientale, par diverses stylisations (程 式), établit un sentiment de jeu scénique.

Le premier s'efforce de faire revivre sur scène la réalité [zhenshi] de maints détails d'environnement, et le second recourt à l'imagination scénique.

L'un compte surtout sur le texte de la pièce pour présenter aux spectateurs les sentiments des personnages, et l'autre se sert des gestes, des regards, des postures ou du pas des acteurs pour révéler aux spectateurs les mouvements intérieurs des personnages.

Alors que le théâtre de tradition occidentale cherche à éveiller la sympathie des spectateurs, à leur faire sentir la psychologie des personnages, le théâtre de tradition orientale les avertit clairement qu'ils regardent un spectacle, et que c'est le jeu des acteurs qui leur fait constamment l'apprécier ${ }^{72}$.

Le zhenshi s'exprime paradoxalement par une exhibition de suppositionnalité et de théâtralité qui s'incarne dans les stylisations de

70. GAO, X. J., 《对一种现代戏剧的追求 》 (La Poursuite d'un théâtre moderne), Beijing, La maison édition du théâtre de Chine, 1987, p. 180.

71. GAO, X. J., « De l'art du jeu de l'acteur », traduit du chinois par Denis Molcanov, dans De la création, Paris, Seuil, 2013, p. 92

72. GAO, X. J., 《我的戏剧和我的钥匙 》, dans Ne pas avoir de-isme, 《没有主义》, op. cit, p. 264-284, traduit en français «Clés pour mon théâtre » par Annie Curien, paru dans Littérature d'Extrême-Orient au xx siècle, Arles, Philippe Picquier, 1993, p. 218-228. 
l'acteur - avec « décohérence » obligée entre le spectacle et l'imitation du réel. Gao ne cherche point le zhenshi sur la scène, car « tout ce qui se passe sur la scène, même si cela peut être très proche du réel, reste faux ${ }^{73}$. En somme, à l'instar de Brecht qui forme sa théorie de la "distanciation " à la faveur de la rencontre du xiqu traditionnel, et toutes choses égales par ailleurs, Gao développe son propre concept de l'« acteur neutre » en retournant vers le mode du percevoir dans l'art du xiqu.

Si l'on est familier et fin observateur du jeu d'acteurs de l'opéra traditionnel chinois, on n'a guère de mal à découvrir que le processus au cours duquel l'acteur entre dans son rôle comporte un intermédiaire, que l'on peut appeler statut d'acteur neutre. Non seulement le jeu de l'acteur est doté d'une dualité, mais il passe par trois étapes, qui sont le moi, l'acteur, et le rôle.

Lorsqu'un acteur d'opéra chinois, qui a une longue expérience sur la scène, s'apprête en coulisses, il commence par purifier son moi; il s'extrait progressivement de sa vie quotidienne et entre en état d'acteur neutre. Quand il a fini de se peindre le visage et de mettre son costume, son allure, sa voix, son expression sont celles d'un autre homme. Dès que tambour et gong s'ébranlent, il se concentre et entre en scène dans la musique en étant rôle. Ce processus de jeu apparaît encore plus nettement lorsqu'un homme est déguisé en femme.

Un homme a sa propre expérience de la vie, caractère, psychologie, comportement et expression en tant que masculin; lorsqu'il joue un rôle féminin, il doit mettre de côté son moi et sa sensibilité sexuelle, et se transmettre à l'acteur neutre. Peu importe qu'il soit un jeune homme ou soit Mei Lanfang lui-même dans la soixantaine, son entrâ̂nement physique et vocal, son costume et son maquillage recouvrent sa caractérisation masculine. Dès qu'il apparaît sur scène, il entre dans le rôle: jeune pêcheuse ou concubine impériale ${ }^{74}$.

Le processus de la transmission tel que Gao le décrit est un processus psychologique qui change le statut, intérieur aussi bien qu'extérieur, via un état d'acteur neutre. Gao a donné l'exemple de la pose de représentation de l'acteur lorsqu'il entre en scène, afin d'éclairer ce processus du changement qui est fondé sur une sorte

73. GAO, X. J., « De l'art du jeu de l'acteur », traduit du chinois par Denis Molcanov, dans De la création, op. cit., p. 93.

74. «Clés pour mon théâtre », dans Littérature d'extrême-orient au xx siècle, op. cit. ; nous traduisons et rajoutons en conformité avec la version chinoise des paragraphes qui ont été éliminés par la traductrice. 
de « convention » - il s'agit d'un accord tacite obtenu par une communication du regard par laquelle la transmission du moi au rôle via l'acteur neutre peut être atteinte.

Marchant au rythme du tambour et du gong, une fois sur scène, il retient son souffle et a les yeux fixés sur la salle; il jette des regards vers les spectateurs, et aussitôt il établit un échange avec eux: à cet instant, son regard est à la fois le rôle, l'acteur et le moi. Allongeons ce processus psychologique afin de le décrire: veuillez regarder moi, Mei lanfang [le moi de l'acteur], ce qui est joué maintenant [l'acteur neutre], c'est la jeune fille Qiujiang [le rôle]! Dès qu'il promène ses yeux, applaudissements et clameurs retentissent dans la salle: le rôle joué par l'acteur est ainsi établi sur la scène. Il joue un rôle, il passe par un échange avec les spectateurs pour être reconnu et s'anime sur scène: la création du rôle commence à se réaliser ${ }^{75}$.

La distanciation brechtienne, comme Patrice Pavis l'analyse, produit une distance entre l'acteur et ce qu'il énonce, visant à « mettre à distance les mots qu'il [l'acteur] prononce, comme pour mieux prendre ses distances par rapport à ce qui est dit et pour faire primer le geste sur le $\operatorname{mot}^{76}$ ". Cette distanciation, destinée à achever de détacher l'acteur de son texte, demande aux spectateurs de ne pas être impliqués dans l'intrigue du théâtre et de ne pas s'abandonner à la sympathie, mais de garder la distance afin d'avoir une attitude critique et lucide envers ce qu'ils voient. Selon Brecht, c'est grâce à la distanciation entre le spectacle et le texte (la langue), grâce à cette perspective critique qu'on parviendra à la vérité sociale.

Il n'en va de même chez Gao: la distance, qui apparaît bien entre l'acteur et le rôle qu'il joue, n'est pas là pour révéler une quelconque vérité, sociale ou idéologique. Elle témoigne de l'existence d'un « entre » (间): « entre l'acteur-homme banal et le "lui” du rôle existe encore une étape, un état particulier que j'appelle l'acteur neutre ".$^{77}$

La distance chez Brecht et la distance chez Gao n'ont rien d'identique. La distanciation de Brecht se focalise sur les deux côtés de la

75. Ibid., nous rajoutons des paragraphes éliminés par la traductrice.

76. Vers une théorie de la pratique théâtrale, Lille, Presses universitaires du Septentrion, 2007, p. 64-65.

77. GAO, X. J., « De l'art du jeu de l'acteur », traduit du chinois par Denis Molcanov, dans De la création, op. cit., p. 105 
distance - l'acteur et le rôle - tandis que l'acteur neutre de Gao prend la mesure de ce qui est entre l'acteur et le rôle. De plus, Gao souligne que cet acteur neutre ne se manifeste pas seulement au moment où l'acteur monte sur la scène: il peut intervenir plusieurs fois durant tout le spectacle en tant que manifestation d'un état spécial, entre le moi de l'acteur et le moi de l'autre (le rôle), un état à défaire la familiarité du moi. À travers cet acteur neutre, Gao montre qu'on est dans le régime de la théâtralité, de la "suppositionalité ». Celle-ci rappelle (paradoxalement, pour un Occidental) le sentiment de la vérité/réel (zhen) chez l'acteur aussi bien que chez les spectateurs en faisant jouer une " convention " tacite, qui ne se réfère pas à la vérité scientifique ni sociale, mais au zhen en train d'être perçu.

\section{L'« entre » (间) comme un passage}

On retrouve l'idée de l'acteur neutre dans les romans de Gao, sous une forme adaptée: c'est le sens même du recours structurel aux pronoms personnels. Cela étant, en l'absence de la « convention " tacite entre l'acteur et les spectateurs, comment basculer le processus psychologique et aussi corporel de la scène au texte, comment passer des trois dimensions spatio-temporelles à deux dimensions? Autrement dit, comment montrer ce qui se joue entre l'acteur-moi et l'acteur-rôle dans le roman?

Lorsque Gao conçoit des structures comme le " je »- « tu »- « elle » de La Montagne de l'âme ou comme le " tu »- «il » du Livre d'un homme seul, il déploie les pronoms personnels comme les différents états du moi. De l'un à l'autre de ces états, il y a des passages où il ne s'agit pas de franchir un creux de distance mais de jeter le pont d'un « entre»(间). Mais qu'est-ce que cet énigmatique « entre »? S'agit-t-il d'un temps et d'un espace entre les états du moi? Ou d'une dimension psychologique?

L'« entre », comme François Jullien le montre ${ }^{78}$, c'est ce qui échappe à la question de l'être, depuis les Grecs. Puisque l'« entre » n’a pas d'" en-soi ", ne peut exister par "soi ", il ne peut non plus être déterminé. Au sein de la pensée grecque, l'« entre » ne servit que

78. JULLIEN, F., L'Écart et l'entre: leçon inaugurale de la chaire sur l'altérité, Paris, Galilée, 2012, p. 49-57. 
d'intermédiaire. Même si "cet "entre" doit néanmoins intervenir pour permettre de penser le changement dans le monde imparfait de la physique ", cet intermédiaire " n’est nécessairement composé que de ses contraires ": il n'a toujours pas d' " en-soi ", d'être ou de nature propre $^{79}$. L' « être » ne peut se déployer sur et dans cet étrange " entre ", condamné à être ignoré, ou plutôt raté par la pensée ontologique.

En revanche, l'" entre " révèle un autre angle possible de l'esthétique et aussi de la philosophie à l'égard du monde - retenu en Chine, face à ce qui est «impensé » en Europe. Vu l'origine du caractère, «閒/间 (entre) » signifie la lumière de la lune traversant la porte: le mot implique d'emblée une fluidité, un va-et-vient, « d'où/ par où procède et se déploie tout avènement ${ }^{80}$ ". En effet, l'" entre " est fréquemment un espace sans forme qui se prolonge indéfiniment entre deux polarités, comme entre le ciel et la terre: entre ce plus haut et ce plus bas de l'univers, les souffles/énergies (气/元气) se transmettent, s'échangent, conçoivent et fécondent la vie. Mais en même temps, les deux termes de la polarité ne correspondent ni à deux " entités " formées et stables, ni à un binôme sujet/objet ou vrai/faux: il s'agit plutôt de deux états qui se transfusent sans cesse l'un dans l'autre, tels que Yin/Yang (阴/阳), dynamique/apathique (动/静), tendu/lâché (张/弛). En d'autres termes, les deux polarités s'ouvrent l'une à l'autre: l'« entre » est un processus qui permet de concevoir cette transformation plutôt qu'une dimension figée.

L'acteur neutre invisible entre les deux états visibles de "l'acteur-homme banal " et du " "lui" du rôle ", fonctionne comme un " entre " qui n'est pas seulement l'intermédiaire qui connecte un moi et un lui (le rôle): il est le passage qui fait que les deux polarités s'ouvrent l'une à l'autre. À la différence de ce que François Jullien propose (l'« entre " comme un outil de " dé-ontologie " rendant possible que l'autre soit en tant qu'autre ${ }^{81}$ ), l'« entre » (间) chez Gao

79. Ibid., p. 53-54.

80. Ibid.p. 54.

81. JULLIEN, F., L'Écart et l'Entre: leçon inaugurale de la chaire sur l'altérité, op, cit., p. 72 : «Car c'est seulement en rouvrant de l'écart et de la distance que l'on peut faire surgir de l'autre, qui ne soit donc pas collé à soi, annexé ou même "aliéné" par soi, parce qu'indexé sur soi, mais qui en soit détaché : qui ne soit pas seulement la projection ou la modification de soi, mais qui puisse effectivement se constituer en "autre" et, par suite, s'établir en vis-à-vis. » 
est ouvert à la fois en dedans et en dehors du moi: il en va ainsi de l'acteur neutre dans le théâtre, aux pronoms personnels dans les romans. Le soi dont François Jullien parle se réfère à la notion d'être dans la philosophie occidentale, où l'autre/l'altérité risque toujours de n'être que la projection du soi, tandis que le moi chez Gao ne se meut pas dans le cadre de l'ontologie: il s'agit plutôt d'un moi qui ne conçoit même pas la notion de l'autre. Créer un autre moi afin de se détacher du moi-même et de s'observer en une attitude lucide, c'est toute la manière du « dé-moi » chez Gao.

C'est la raison pour laquelle "tu/elle " est à la fois l'ombre de " je " et l'autre par rapport au moi dans La Montagne de l'âme. En même temps, ceux qui se trouvent des deux côtés de l'" entre " ne sont pas des substances comme les conçoit l'ontologie, mais des états d'existence qui se transforment sans cesse. Ainsi « je », «tu/ elle » apparaissent plutôt en tant que différents états du moi, plutôt que comme des caractères personnels. On verra comment les pronoms personnels incarnent les divers états du moi: « je » signifie un état de la conscience errant et percevant dans le monde réel; " tu » représente un état psychologique qui mêle l'imaginaire de soimême et de l'autre (masculin); " elle " révèle l'image des femmes en montrant un état affectif qui s'identifie inconsciemment à l'autre (féminin).

Dans Le Livre d'un homme seul, on l'a vu, la structure binaire de « tu » et « il » se substitue à la structure triangulaire de " je », « tu » et " elle " de La Montagne de l'âme — « je " y a disparu. Pourtant, cette « exclusion " du « je » ne signifie pas une divergence du moi; au contraire, c'est plutôt « je " qui est divisé et représenté en « tu » et en « il " - tandis que ces derniers incarnent deux états du moi dans le temps du passé et du présent. De même concernant " elle " qui se retire de la réunion des figures de femmes dans La Montagne de l'me, remplacée par les femmes concrètes ayant leurs propres noms: Marguerite, Sylvie... Mais les protagonistes féminins se présentent toutes en même temps comme le miroir des silhouettes du moi. Le souvenir de Marguerite contraste avec le passé de «tu », ainsi bien que « il "; l'amour sans engagement de Sylvie pour " tu " reflète l'amour profond des filles pour «il ». Autrement dit, les femmes révèlent l'image du moi dans ses différents états. 
Les états du moi ne signifient pas des états figés: ils s'ouvrent l'un à l'autre. Dans La Montagne de l'âme, « je " et « tu " échangent leurs statuts; dans Le Livre d'un homme seul, « tu » et " il " passent de l'un à l'autre état, et même se superposent. Car l'« entre " (间) qui s'ouvre entre eux n'est pas figé non plus. Pour ainsi dire, l'« entre " permet à Gao de construire dans les romans les états du moi et, en même temps, les états du moi donnent la possibilité d'avoir à disposition l'« entre » qui les distingue.

Dans La Montagne de l'âme, l'" entre " soutient un va-et-vient entre les différents états du moi pris dans les différents temps et espaces: l'« entre » n'apparaît pas seulement entre deux moi (« tu » et « elle ») qui se projettent sur les autres, mais aussi entre les deux polarités d’un moi dé-familiarisé ( « tu ») et du moi renforcé (« je »). Au début du roman, la distance entre « tu » et « je » est immense: les récits de « tu/elle » tendent vers la polarité d'un moi qui s’identifie à autrui, dont les deux figures en dialogue parlent; ils sont plutôt les histoires d'autres, des contes lointains ou des légendes du folklore; alors que les récits de « je » tendent vers la polarité du moi renforcé, qui est déployé sur ses propres expériences en voyageant le long du fleuve Bleu. Mais lorsque l'ordre de l'alternance entre les récits de " tu/elle " et ceux de " je » est interrompu, dans les chapitres 31 et 32 , les histoires de " tu/elle » et celles de « je " tendent dès lors à s'approcher du souvenir de l'enfance. À partir du chapitre 52, " elle » commence à s'estomper, étant l'ombre de " tu », c'est-à-dire un moi se projetant sur l'autre: « je » et « tu » sont de plus en plus intimes.

À la différence de ce qui se passe dans La Montagne de l'âme, l'« entre " (间) révèle dans Le Livre d'un homme seul un passage indirect entre l'état du moi dans le temps du passé et son état du présent, la perception de leur entre-deux étant évoquée par un tiers. Marguerite, fille d'une mère juive et d'un père allemand, qui « a besoin de porter sur elle les souffrances des juifs et la honte de la nation germanique ${ }^{82}$ " mène " tu " à se remémorer les expériences douloureuses vécues durant la révolution culturelle. Aussi, lorsque « il » rappelle son enfance ( " Il avait bien connu une autre vie, mais

82. GAO, X. J., Le Livre d'un homme seul, op. cit., p. 91. 
par la suite il avait fini par l'oublier ${ }^{83}$. »), « tu » le rejoint (à partir du troisième récit de « il ", au chapitre 5):

Plus tard, sa mère lui avait acheté une collection de livres pour enfants, Les cent mille pourquoi, dont il avait lu chaque volume sans que cela lui laisse la moindre impression, et ses doutes premiers à l'égard du monde étaient restés enfouis en lui.

De la lointaine enfance, comme une brume, comme une fumée, ne subsistaient plus dans la mémoire que quelques taches brillantes qui émergeaient peu à peu lorsqu'il en évoquait un fragment, enfouies par le temps dans la mémoire, comme un filet sort de l'eau maille après maille - il suffit d'en tirer un bout pour que tout le reste suive ${ }^{84}$. Des moments et des faits d'époques différentes resurgissaient en même temps, et il était impossible de savoir par quel bout les prendre, impossible de trouver le fil conducteur pour les faire remonter à la surface et les classer; il était impossible souvent de les comprendre. La vie humaine est un filet que tu voudrais défaire, nœud après nœud, mais tu n'obtiens en fait qu'une boule de fils emmêlés. Et tu n’arrives pas à régler cette addition stupide qu'elle représente ${ }^{85}$.

L'image brumeuse de l'enfance lointaine est en effet la transition entre « il » et « tu », comme le passage/l'« entre », dont l'expérience commune évoque la même perception. En fait, c'est un roman de double rétrospection dont le temps du passé (la révolution) est rappelé par un autre temps du passé (l'enfance) et non par le temps présent. Le présent n'est pas le révélateur du passé, mais le contraste/reflet ou le « dialogueur » du passé. Il y a ainsi un « entre » (间) entre le passé et le présent mais il ne se présente pas comme une transition temporelle entre eux. Prenons des passages de la fin du chapitre 7 pour exemple:

83. Ibid., p. 20

84. Nous modifions la traduction française pour souligner le non-sujet dans la langue chinoise (voici la traduction française: "De sa lointaine enfance, comme une brume, comme une fumée, ne subsistaient plus dans sa mémoire que quelques taches brillantes qui émergeaient peu à peu lorsqu'il en évoquait un fragment, enfouies par le temps dans sa mémoire, comme un filet sort de l'eau maille après maille - il suffit d'en tirer un bout pour que tout le reste suive. ») Car dans langue chinoise, le sujet « qui » ou « de qui » peut être absent, si bien que la phrase peut se trouver plus floue ou ambiguë, tandis que dans la langue française, le sujet est indispensable.

85. GAO, X. J., Le Livre d'un homme seul, op. cit., p. 62. 
Après des combats de nuit qui avaient duré jusqu'au matin, le ciel commençait à blanchir. Il était allé aux toilettes se laver le visage. L'eau fraîche lui avait éclairci les idées [...] Pour la première fois il prenait conscience qu'il était sans doute un ennemi "tapi dans l'ombre » et que s'il voulait survivre, il devait obligatoirement se dissimuler derrière un masque.

- Attention à la fermeture des portières, prochain arrêt Prince Edward Station.

L'annonce est faite en cantonais, puis en anglais. Tu t'es endormi, et tu as raté la station. [...] Tu devras faire demi-tour au prochain arrêt, rentrer à ton hôtel dormir un peu, tu ne sais plus où tu t'es réveillé cette nuit, tu étais sur un lit avec une Occidentale couchée à côté de toi. Tu es déjà inguérissable, et tu n'es plus seulement un simple ennemi, tu te précipites vers l'enfer, car pour lui, le souvenir est justement comme un enfer ${ }^{86}$.

Comme le souvenir d'enfance lie « il » et « tu " dans le même chapitre, le souvenir de la révolution culturelle met les deux temps du passé et du présent en parallèle — ou plutôt, il y a une juxtaposition des états de moi dans le passé et le présent. Il ne s’agit certainement pas d'une juxtaposition du rêve et du réel: pas seulement parce que le souvenir de " tu » est beaucoup plus lourd qu'un rêve, mais aussi parce que les deux narrateurs « il " et « tu " ne se trouvent pas dans les différents temps qu'ils racontent (passé et présent), mais habitent dans la même perception d'ici et maintenant.

Paul Ricœur a repris la distinction entre le temps du raconter et le temps raconté introduite par Günther Müller, puis réinterprétée par Gérard Genette, pour analyser le jeu avec le temps chez Proust ${ }^{87}$. Chez ce dernier, comme Ricœur le relève, le narrateur " je ", qui s’adresse souvent au lecteur, ne recouvre pas le héros « je »; en effet, le temps « présent » où le narrateur raconte n’est pas le même temps que « le futur dans le passé ${ }^{88}$ " dont la distance ne permet pas d'interpréter le temps perdu en fonction de quelque temps retrouvé ${ }^{89}$. Dans Le Livre d'un homme seul, même si les narrateurs « il » et « tu »

86. Ibid., p. 79.

87. RıcœUR, P., Temps et récit 2. La configuration dans le récit de fiction, Paris, Seuil, 1984, p. 143-159.

88. Ibid., p. 252.

89. Ibid., p. 246-286. 
ne se recouvrent pas non plus avec les états de « il » et de « tu » dans leurs temporalités propres, cette distinction n’est pas révélée par l'avertissement de narrateurs « il »/« tu » s'adressant aux lecteurs, mais par l'« entre " entre " tu » et « il » eux-mêmes.

Il y a ainsi deux niveaux de l'« entre» (间): dans l'un, c'est la juxtaposition de l'état de «il » et de " tu » dans le passé et le présent; dans l'autre, il s'agit paradoxalement du croisement de "il " et de « tu » qui se détachent des états du moi dans le temps. Cet « il » qui se rend compte qu'il est sans doute un ennemi pour les autres (l'ennemi de la classe prolétarienne) mais aussi pour lui-même (il se cache désormais derrière le masque), n'est pas le "il » de la révolution culturelle; de même, ce " tu " qui réalise qu'il est aussi l'ennemi de lui-même, n'est pas non plus le narrateur " tu » à Hong Kong. Pourtant, tous les deux (cet «il » et ce " tu ») représentent les perceptions d'ici et maintenant - de ce point de vue, «il " et « tu " sont interchangeables: " tu n'es plus seulement un simple ennemi, tu te précipites vers l'enfer, car pour lui, le souvenir est justement comme un enfer ${ }^{90}$."

90. GAO, X. J., Le Livre d'un homme seul, op. cit., p. 79 ; marquage en gras par nous. 



\section{4 \\ Mon regard n'est pas en moi}

Je suis celui qui suis.

La Bible, Exode 3 :14

JE est un autre

Rimbaud, Lettres du voyant

\section{MON REGARD EST EN DEHORS DE MON CORPS Le corps et l'œil}

Dans le processus de perception, si c'est le vrai sujet du sentir, incarné en un autre moi, qui me fait éprouver l'expérience de la sensation, mais ne me fait pas connaître l'auteur de cette perception, et qui fonctionne dans la dépendance de mes organes, mais déborde en même temps de mon corps, où se trouve alors le corps du moi? Sa place est-elle au dedans ou au dehors du moment où le vrai sujet perçoit?

Ce corps est à la fois abstrait et concret, à la fois évident et estompé dans les romans, les pièces de théâtre et les peintures de Gao, qui se pose d'emblée des questions sur lui-même: de quoi s'agitil, avec mon corps? Comment et par qui, mon corps est-il éprouvé?

Avec ce corps, il ne s'agit pas, chez lui, d'une chair objective appartenant au moi, mais plutôt d'une chair désirante et souffrante qui est sensible et transformable selon ce qui est perçu. Commentateur assidu de ses propres ouvrages, Gao explique ses théories - de la narratologie des pronoms personnels, de l'acteur neutre, du troisième œil (tiers en observation) —, mais il ne parle jamais du corps, qui s'impose pourtant comme une instance très importante chez lui, notamment dans les créations postérieures à son installation en France. 
De même, le lien naturel entre le corps et le moi est à la fois certain et incertain: le corps en moi est comme un autre dans lequel je ne m'éprouve pas. Dans Le Livre d'un homme seul, Marguerite, qui a été violée à l'âge de treize ans par son professeur de peinture, ne se rend compte que son corps a été abusé que deux ans plus tard, au moment où elle rencontre le regard d'une autre fille de cet atelier:

Je parle de ce regard qu'elle a porté sur mon corps, je me hais moimême, il n'y avait pas qu'elle qui me haïssait, je me suis vue moimême à travers son regard, je le hais, et je hais ce corps qui est trop tôt devenu le corps d'une femme ${ }^{1}$.

Marguerite éprouve son corps par la vision et non pas par le contact. Le corps est aussi pour Marguerite comme une voie par laquelle surgit la conscience de soi: au moment où elle éprouve son corps, elle se voit elle-même. Lorsque le corps est éveillé par une vision extérieure, il réveille aussi une vision intérieure. En effet, chez Gao, le regard introspectif, c'est-à-dire l'auto-observation passe toujours par le corps - le sien propre ou celui d'autrui. Cette tendance s'entrevoit déjà dans la dernière pièce de théâtre qu'il ait écrite en Chine, L'Autre Rive: l'ombre qui représente la conscience lucide de l'« homme " permet à ce dernier de découvrir, à travers les corps des mannequins en plastique, sa propre nature et son propre désir. Dans La Montagne de l'âme, " tu » et "elle " qui sont les ombres de "je " reflètent un autre corps du moi - il s'agit d'une chair sexuée qui est ignorée par «je ». Dans sa première pièce de théâtre écrite en français en 1991, Au bord de la vie, l'auto-observation d'ELLE - l'héroïne de cette pièce monologique - commence par le détachement du bras et du pied de son corps. Dans sa pièce suivante écrite en 1992, Dialoguer-interloquer, la communication entre les deux protagonistes inconnus passe d'abord par leur amour physique; c'est à partir de là que leur discussion se déroule. Dans Le Livre d'un homme seul, le corps apparaît encore comme le premier intermédiaire entre " tu » et Marguerite, entre Marguerite du présent et Marguerite du souvenir, aussi bien qu'entre les deux moi, le « tu » du présent et le " il » du passé: le corps fait naître leur communication et leur confère une vision intérieure, telle qu'ils puissent se voir eux-mêmes.

1. GAO, X. J., Le Livre d'un homme seul, op. cit., p. 161. 
Il apparaît, à travers les ouvrages de Gao, que se connaître soimême n'est pas une action indépendante du sujet lui-même, ou encore, que le sujet n'est pas autonome: le sujet voyant n'est pas ce qui s'examine soi-même, c'est un certain emprunt du corps - de soi qui est comme un autre, ou de l'autre qui permet d'accomplir la quête du soi. Le corps ne représente pas seulement la voie incontournable d'accès à la vision du moi, il est aussi le révélateur de la séparation entre le moi et sa vision.

Ces paradoxes peuvent être éclairés par une analyse d'Au bord de la vie, pièce en un acte ou plutôt en un monologue, au fil duquel l'héroïne ELLE raconte sa vie. En ouverture, ELLE se plaint de l'infidélité et de l'hypocrisie de l'homme qui est initialement là et reste silencieux. Elle supplie enfin cet homme qui est en train de disparaître de ne pas la quitter. Puis ELLE tourne avec effort l'épaule de l'homme, et se rend compte qu'il n'y a qu'un complet d'homme accroché au portemanteau. ELLE comprend qu'elle tue cet homme:

ELLE dit ne jamais avoir pensé que cela se terminerait ainsi, ne jamais avoir imaginé qu'ELLE tuerait son homme, son préféré, son amour, son mal, ce salopard, cette ordure, son possédé2.

Mais ELLE le nie tout de suite:

Ce n'était qu’un cauchemar. ELLE dit qu'ELLE est réveillée à présent, et qu'ELLE a froid.

Gao a dit que cet homme sans parole et les autres figures masculines (le clown, l'homme sur des échasses et le vieux) " ne sont que les projections des états psychiques de la femme » ou " un écho aux états d'âme de l'héroïne ${ }^{3}$ ». Les figures des hommes ne représentent qu'une absence du corps réel et par cette absence, ELLE retourne son regard vers elle-même. Puis, ELLE constate que son pied et son bras commencent à se détacher de son corps:

Inutile de se regarder dans le miroir: ELLE connaît déjà son visage défait. [...] Que peut-ELLE encore espérer? Son corps abandonné n’a plus la moindre envie.

(Elle serre davantage la cape contre elle, les yeux et la tête baissés. Un pied écorché sort de dessous sa robe. Surprise, elle recule)

2. GAO, X. J., Théâtre 1, Belgique, Lansman, 2000, p. 67.

3. GAO, X. J., «Le Potentiel du théâtre », dans De la création, op. cit., p. 74. 
ELLE ne peut pas y croire. Ce n'est pas vrai! (S'inclinant pour examiner) Ce n'est pas le sien! Il faut qu'ELLE sache si c'est la réalité... ou seulement un cauchemar.

(Le pied s'allonge, se détache finalement sous la robe et tombe par terre. Elle recule encore d'un pas, le souffle coupé)

ELLE ne sait plus, à cet instant, si ELLE est vivante ou morte, si ELLE respire encore, si c'est une simple illusion? Ce corps de chair n'est-il qu'une apparence ou une affabulation?

(Elle se pince le bras. Une main pâle aux doigts fins, aux ongles vernis de nacre, sort de la cape)

Quelle horreur! (Stupéfaite et haletante) ELLE veut savoir si sa stupeur est réelle ou si ELLE a cru seulement s'effrayer. De toute façon, ELLE sait qu'ELLE doit au moins une fois affronter la mort, vivre cette souffrance, expérimenter cette douleur. Alors seulement, il lui sera possible de s'ancrer dans la chair de l'existence, d'appréhender la vie et de choisir son contraire. ELLE souffre de cette fouille au plus intime d'ELLE-même, en butte à sa propre quête, cherchant désespérément confirmation de la femme... ou seulement de ce corps de chair; mais rien ne se révèle au-delà de l'apparence illusoire de cette peau disloquée.

(Tout un bras se détache. À part la main, le reste est peint comme le pied. Elle fuit)

Non! ELLE ne peut continuer de se démembrer, de se mutiler. Il faut fuir immédiatement cette chambre terrible... Mais ELLE s'est enfermée. Comment a-t-ELLE pu être si stupide?

(Elle cherche la clef parmi les vêtements de l'homme, puis dans sa boîte à bijoux, tout en contournant avec frayeur le pied et le bras tombés ${ }^{4}$ ).

C'est à partir de ce moment qu'ELLE découvre son propre corps, qui ne lui appartient plus et qui lui procure cependant une vision intériorisée d'elle-même, par remontée dans son enfance et regard en arrière. Comme Henry Zhao le montre, le regard ne se projette plus de l'intérieur à l'extérieur du personnage, mais plutôt de l'extérieur vers l'intérieur ${ }^{5}$. Cette vision intérieure semble se détacher peu à peu des yeux d'ELLE, qui se rattache à son corps. Les figures féminines (la silhouette d'une femme, la bonzesse et le corps de la femme décapitée, qui se donnent à voir successivement sur la scène tout au long du monologue d'ELLE) font résonner des figures masculines

4. GAO, X. J., Théâtre 1, op. cit., p. 67-68.

5. Z HAO, H., 《高行健与中国实验戏剧-建立一种现代禅剧》, op. cit., p. 153. 
mais, en contraste avec l'absence du corps de ceux-ci, elles révèlent plutôt un autre corps d'ELLE situé en dehors d'ELLE.

Car si le pied et le bras se détachent " volontairement " d'ELLE, c'est qu'une certaine « subjectivité/entité » existe en dehors du corps réel. Il y a deux niveaux du corps d'ELLE: le corps en ELLE, c'està-dire ce qui est en train de parler et regarder; le corps qui n'est pas en ELLE - c'est le sujet à la fois voyant et visible, représenté par les figures féminines: la silhouette d'une femme s'incarne en l'ombre d'ELLE, la bonzesse représente son corps désirant mais en même temps repentant, et la femme décapitée qui possède paradoxalement une vision pénétrante a la faculté de s'observer soi-même.

Le corps d'ELLE et ELLE ne sont plus cohérents ni uniformes. ELLE n'est pas vraiment le sujet de l'acte de connaître son corps: ELLE éprouve son propre corps à l'aide de l'apparition des figures féminines. ELLE et les figures féminines entrent en rapport à partir d'un " même " corps mais s'écartent en même temps les unes des autres du fait de la diversité des perceptions qu'elles éprouvent. Leur relation émerge d'une manière assez subtile et allusive: chaque fois qu'ELLE remonte dans son enfance ou raconte sa vie, son regard s'attache à l'apparition de l'une des figures féminines et s'implique involontairement dans l'exhibition de leur corps. Pour ELLE, la quête du soi commence par la perception visuelle de son corps et aborde inévitablement la question du désir.

Il y a aussi ce regard d'un autre qui fait qu'ELLE éprouve son corps désirant:

(Un grand homme monté sur des échasses, vêtu d'une cagoule blanche à capuchon, apparaît derrière elle. il lui tend un bras très long et lui montre un oil géant dans sa paume)

Terrifiée, en sueur, le corps tremblant, le cœur battant, paralysée, stupéfaite, à la fois excitée et torturée. ELLE a la brusque révélation de l'origine de son mal: tout vient de cet œil-là, qui a vu sa timidité, sa culpabilité, sa maladresse, ses caprices... son sado-maso-fardeau qui l'a impliquée dans ces souffrances depuis son adolescence, et qui guide finalement toute son existence!

(Elle se tourne et s'efforce de s'en débarrasser. L'œil, tenu dans la paume de l'homme, la surveille d'en haut, ne la lâche pas. Elle crie) Mais non! 
(Elle s'enfuit en courant. L'homme ne la suit pas, la fixe seulement par cet œil tenu dans la paume de sa longue main. Quand elle s'arrête, l'œil se trouve à nouveau derrière elle, au-dessus de sa tête)

Non!

(Elle s'agenouille, se cache la tête dans les mains et s'allonge par terre. L'homme lui met la main au-dessus de sa tête allongée. Elle murmure ${ }^{6}$ ).

Cependant, si les figures masculines ne sont que la projection d'ELLE, alors l'œil dans la paume qui représente un regard érotique et voyeuriste de l'homme, qui la force à envisager et à sentir son propre corps, est aussi un regard imaginaire d'ELLE-même. Cette ambiguïté, entre regard d'ELLE et regard de l'autre, atteste que la vision d'ELLE est une vision passive à la fois et active. Ce " métissage " de la vision est encore accentué à la fin de la pièce, lorsque le corps de la femme décapitée tente plusieurs fois de lui montrer l'œil d'ELLE-même, mais hors d'ELLE:

(Le corps décapité s'approche d'elle et tend une main devant ses yeux. Elle manifeste sa surprise)

Cette fois, ELLE voit apparaître devant ses yeux un œil de femme!

(En reculant, le corps décapité retire la main. Elle se lève, réfléchissant tristement)

ELLE ne sait pas si ELLE voit sortir d'ELLE sa propre âme... (Le corps tend la main devant ses yeux)... ou si, par cet œil hors d'ELLE, ELLE se voit ELLE-même...

(Le corps retire la main. La femme tourne, tête baissée)

ELLE se voit maintenant, allongée dans les ténèbres, onduler légèrement et glisser sans poids; ELLE voit son propre corps, nu, sur le point d'être immergé, à peine porté par la crête des vagues noires, mais invisibles, puis plonger profondément. Quand une nouvelle vague montante la soulève, ELLE se livre aussitôt à la chute suivante, plus profonde encore, et plus noire...

(Le corps décapité tend une dernière fois la main)

Ce regard tout froid, la guette perpétuellement! (Criant)

Qui es-tu? Un spectre ou une image de cauchemar?

(Elle s'efforce de se débarrasser de ce corps tantôt visible, tantôt absent, qui montre un bras et une jambe, attirant la femme et la faisant tourner autour de lui. Elle se fâche ${ }^{7}$ )

6. GAO, X. J., Théâtre 1, op. cit., p. 70-81.

7. Ibid., p. 82-83. 
En réaction à la perception transmise par le corps, qui est à la fois perception d'ELLE-même et perception de la femme décapitée, l'exercice de sa capacité de vision par ELLE combine la volonté de regarder et la passivité contrainte. De son côté, le corps qui n'est pas en ELLE, n'est pas non plus un objet qui reçoit passivement la perception, mais plutôt un sujet actif. Les émotions et les stupéfactions qui arrivent sur ELLE, semblent venir du dehors d'ELLE, et la font les suivre: en d'autres termes, ELLE n'est pas le maître de sa propre vision.

En outre, le corps d'ELLE n'est pas une présence existant a priori dans sa propre vision. C'est l'œil de l'autre ou l'œil d'ELLE mais placé en dehors d'ELLE qui fait qu'ELLE voit son corps désirant et souffrant - autrement dit, son corps "subjectif ". À partir de là, son propre corps lui est à la fois invisible (en tant que chair percevante) et visible (le corps existant). De même, les représentations de son corps, les figures féminines, sont visibles mais aussi invisibles pour ELLE, puisque celle-ci les voit sans les reconnaître.

\section{L'invisible du visible}

La séparation du corps et d'ELLE, qui révèle la rupture entre le moi et sa vision, fait naître pourtant une autre vue qui ne correspond pas à un regard normal mais implique la partie invisible dans le regard habituel. Dans Au bord de la vie, l'œil dans la main du corps décapité marque cette rupture du moi et de sa vision et aussi, en ELLE, cet invisible pour elle-même: il ne s'agit pas de ce qui est à l'opposé du visible, mais de ce qui surgit derrière le visible comme une profondeur de champ: cette profondeur et le visible se complètent mutuellement.

Lorsque Merleau-Ponty travaille sur "l'énigme de la vision ", il parle d'une attitude naturelle de la vision dans laquelle je fais cause commune avec mon regard et me livre par lui au spectacle:

Alors les parties du champ [visuel] sont liées dans une organisation qui les rend reconnaissables et identifiables. La qualité, la sensorialité séparée se produit lorsque je brise cette structuration totale de ma vision, que je cesse d'adhérer à mon propre regard et qu'au lieu de vivre la vision je m'interroge sur elle, je veux faire l'essai de mes 
possibilités, je dénoue le lien de ma vision du monde, de moi-même et de ma vision, pour la surprendre et la décrire ${ }^{8}$.

Cette « qualité, sensorialité séparée » se produit au moment où je ne suis plus le maître de ma vision; il s’agit sans doute d'un invisible de « droit » dans le règne du visible:

Ce qui s'oppose à ce que je me voie c'est un invisible de fait d'abord (mes yeux invisibles pour moi) mais, par-delà cet invisible (dont la lacune se comble par autrui et ma généralité) [est] un invisible de droit: je ne puis me voir en mouvement, assister à mon mouvement ${ }^{9}$.

Les yeux et le corps qui sont derrière et inséparables du regard et soutiennent la vision, ne sont pourtant pas dans sa propre vision. Pour ainsi dire, le visible repose sur l'invisible et non pas sur le visible lui-même, comme l'œil est invisible pour lui-même. Ainsi:

Quand je dis donc que tout visible est invisible, que la perception est imperception, que la conscience a un "punctum caecum ", que voir c'est toujours voir plus qu'on ne voit, - il ne faut pas le comprendre dans le sens d'une contradiction - Il ne faut pas se figurer que j'ajoute au visible parfaitement défini comme en Soi un non-visible (qui ne serait qu'absence objective) (c'est-à-dire présence objective ailleurs, dans un ailleurs en soi) - il faut comprendre que c'est la visibilité même qui comporte une non-visibilité — dans la mesure même où je vois, je ne sais pas ce que je vois (une personne familière est non définie), ce qui ne veut pas dire qu'il n'y ait là rien, mais que le Wesen [essence] dont il s'agit est celui d'un rayon de monde tacitement touché [...] — Le monde perçu (comme la peinture) est l'ensemble des chemins de mon corps et non une multitude d'individus spaciotemporels — L'invisible du visible ${ }^{10}$.

Certes, Merleau-Ponty ne traite pas seulement l'invisible comme " la doublure et la profondeur ${ }^{11}$ ", mais l'aborde dans le sillage d'une réflexion sur la réversibilité: le corps de l'activité (voyant) ne peut s’accomplir que dans une proximité constante à un corps de la passivité (être vu):

8. Merleau-Ponty, M., Phénoménologie de la perception, dans Maurice Merleau-Ponty, Euvres, op, cit., p. 917.

9. Merleau-Ponty, M., Le Visible et I'Invisible, suivi de Notes de travail, Paris, Gallimard, 1964, p. 308.

10. Ibid., p. 300

11. Ibid., p. 195. 
Le corps est " chose sentante ", " sujet-objet » [...] Si la distinction du sujet et de l'objet est brouillée dans mon corps, elle l'est aussi dans la chose, qui est le pôle des opérations de mon corps, le terme où finit son exploration, prise donc dans le même tissu intentionnel que lui ${ }^{12}$.

Nous trouvons chez Gao une telle réversibilité: celle d'un même corps qui est représenté par différents personnages, « je », « tu ", « elle » et « il ».

C'est à partir d'ici que Gao croise Merleau-Ponty: l'invisible qui se fait en nous et qui implique paradoxalement une certaine nonsubjectivité dans le geste de regarder, ne révèle pas une perspective existentialiste qui prête le néant à la subjectivité. Comme la profondeur du visible, l'invisible donne une réflexion qui ne porte pas sur le moi agissant, mais sur une perception qui ouvre «sur des horizons et des lointains, c'est-à-dire sur un monde qui est là pour elle d'abord, et à partir duquel seulement elle se sait comme un titulaire anonyme vers lequel cheminent les perspectives du paysage $^{13}$ ». Aussi, le corps qui contient à la fois l'invisible et le visible et qui révèle la réversibilité de l'activité et de la passivité, déchiffre le secret de la vision en déployant et percevant soi-même.

Mais c'est aussi à partir de ce point que l'approche de Gao se distingue des idées de Merleau-Ponty. Chez celui-ci, la pensée de l'invisible appelle une autre compréhension pour une « nouvelle ontologie » au sens sensible: l'invisible " n'est pas un vide ontologique, un non-être [...], c'est le zéro de pression entre deux solides qui fait qu'ils adhèrent l'un à l'autre ${ }^{14}$ ". Tandis que chez Gao, l'invisible ne conduit pas à la recherche d'une nouvelle ontologie, ne s'implique pas non plus dans le sillage d'une réflexion sur l'être: bien que l'invisible du visible appelle aussi la réversibilité du corps dans le processus de la vision, il s'agit d'une manière de poursuivre le désir et la perception qui ne sont pas seulement éprouvés mais encore s'éprouvant eux-mêmes, afin de frayer une nouvelle voie pour atteindre un vrai moi au niveau de la perception.

12. Merleau-Ponty, M., Sur Husserl. Le philosophe et son ombre, dans Maurice MerleauPonty, Euvres, op. cit., p. 1275.

13. Merleau-Ponty, M., Le Visible et I'Invisible, suivi de Notes de travail, op. cit., p. 42. 14. Ibid. 
Dans La Montagne de l'âme, " je » est marqué par une vision projetée vers le monde extérieur et réel, et n’envisage pas le désir réel de son corps. Pendant son long voyage, «je " rencontre plusieurs filles, mais n’ose jamais obéir à son désir. Dans le village des Miao, « je » se promène comme un touriste curieux, pour voir la fête traditionnelle du chant d'amour; soudain, une jeune fille l'appelle:

Je croise un regard fixe et langoureux dans l'obscurité, je suis fasciné et mon cœur se met à battre [...] Je sais que, sur un simple geste de ma part, elle me suivra et se blottira contre moi. [...] Je ne peux supporter cette confrontation qui se prolonge et je hoche la tête avec conviction en riant bêtement. Trop peureux, je me retourne et je m'éloigne sans même oser jeter un regard ${ }^{15}$.

Même si ce genre d'appel de l'amour est «justement ce dont j'ai le plus rêvé ", le regard de la fille Miao ne déclenche en rien un même regard de "je " qui répondrait à son propre désir; au contraire, « je " réprime un tel regard et s'enfuit. Cette chair du désir, absente chez « je ", s'offre à voir chez « tu » et " elle " qui vagabondent dans un monde irréel, apparaissent d'emblée comme deux figures désirantes, et ne parlent que d'amour, de trahison, de relation extraconjugale à travers les histoires des autres et d'eux-mêmes. Ils représentent par leurs propres corps le désir et le fantasme sexuel de " je » - ce dernier ne les voit pas et n'ose pas les voir; de plus, ils lui transfèrent la sensation sexuelle que « je " n'éprouve jamais, par leurs corps qui sont l'ombre de son corps à lui.

L'invisible du désir et la réversibilité du voyant visible sont mis en scène aussi par le truchement d'une autre histoire, issue d'un ancien conte de la dynastie Jin (晋) écrit par Tao Yuanming ${ }^{16}$. Cette histoire occupe le chapitre 24 de La Montagne de l'âme; elle est comme un archétype, et reparaît sous des formes variées dans les autres œuvres de Gao. Une nonne qui loge chez un général prend un bain de deux heures à chaque début d'après-midi. Un jour, le général jette un clin d'œil par curiosité vers la porte entrebâillée, il voit la nudité de la nonne qui est en train de se laver:

15. GAO, X. J., La Montagne de l'âme, op. cit., p. 313-341.

16. TAO, Y. M. (陶渊明), 《搜神后记·卷二 》(Shoushen Houji), Shanghai, Shanghai guji chubansh (上海古籍出版社), 2012. 
Le visage [de nonne] tourné vers l'entrée, le teint rose et les dents blanches, les joues poudrées et la nuque comme du jade, l'épaule lisse et les fesses rondes, véritable figurine de jade. Il s'écarta à la hâte et revint dans la grande salle, pour reprendre ses esprits.

Le bruit de l'eau retentissait encore dans la chambre, l'attirant malgré lui. Retenant son souffle, il colla son œil à la fente de la porte et entrevit dix doigts très fins qui s'ouvraient pour masser deux seins pleins, blancs comme neige, embellis par deux boutons de fleurs prêts à éclore. La peau humide se soulevait légèrement et une fine ligne se dessinait du nombril au pubis. Le général en tomba à genoux de surprise, incapable de se relever.

Puis il vit deux mains blanches sortir des ciseaux de la cuvette, refermer les deux lames et les planter avec force dans le ventre. Le sang frais, rouge foncé, jaillit sous le nombril. Terrifié, il n'osait bouger et ferma les yeux.

Un instant plus tard, le bruit de l'eau reprit. Il rouvrit les yeux et, fasciné, vit la nonne au crâne chauve qui baignait dans le sang, mais ses mains ne cessaient de s'agiter pour sortir ses viscères et les placer dans la cuvette!

[...]

De ses deux mains ensanglantées, elle se saisit de ses intestins qu'elle massa du bout des doigts, lava minutieusement, puis elle passa un long moment à les disposer sur ses avant-bras. Lorsqu'elle eut fini de les laver, elle arrangea ses entrailles, les souleva et les replaça dans son ventre. À l'aide d'une louche pleine d'eau, elle se lava successivement les bras, la poitrine, les plis de l'aine, les pieds et même les orteils, comme si de rien n'était ${ }^{17}$.

Le général effrayé questionne finalement la nonne, et celle-ci lui répond: "Seigneur, si vous prétendez au trône, votre sort sera tel que ce que vous venez de voir. " Le général se rend compte que le corps qu'il voit est en effet le reflet de son propre corps dans le futur - il sera condamné à mort s’il suit son désir du pouvoir. Il y a chez le général un désir visible pour le corps de la nonne et aussi un désir invisible pour soi-même (le pouvoir); son voyeurisme effectue un échange inter corporel en voyant son propre désir dans le corps de l'autre.

On rencontre encore cette histoire dans Au bord de la vie et dans une autre pièce de théâtre, La Cité morte, achevée en 1991. À la différence de l'histoire originelle où l'autodissection de la nonne reflète le désir d'usurpation du général, le corps de l'autre est transposé en

17. GAO, X. J., La Montagne de l'âme, op. cit., p. 384-385. 
corps de soi-même; dans Au bord de la vie, l'autodissection de la bonzesse qui est un autre corps d'ELLE constitue un miroir transparent pour ELLE même:

(Une bonzesse au visage livide, le crâne rasé, les yeux baissés, les mains posées l'une sur l'autre, vêtue d'un kasaya gris - longue et large robe croisée - fait son apparition, assise en tailleur sur un coussin de jonc) ELLE voit maintenant la Déesse de la Miséricorde, les mains jointes, assise sur des fleurs de lotus.

(Elle s'approche avec précaution)

Mais les lotus, de plus près... des joncs. Et la déesse... une bonzesse, les yeux fermés, en train de méditer.

(Elle l'observe minutieusement)

Elle a les sourcils légèrement rapprochés. Dans ses mains... une paire de ciseaux!

(La bonzesse lève lentement les ciseaux, se les enfonce d'un coup dans le ventre)

Oh!

(Elle rampe par terre. La bonzesse se plie d'abord en deux puis, les lèvres pincées, se relève, bascule la tête à la renverse)

Pourquoi tout cela? Pour rien, voilà!

(Renversée en arrière, la bonzesse repose les ciseaux sur le coussin. La femme recule)

Mais que fait-elle? Et qu'est-ce qu'elle veut? Elle se dissèque toute seule! (La bonzesse extirpe les viscères de son corps et les dépose dans une assiette devant elle. Puis elle les pétrit. La femme reste immobile un moment puis respire profondément)

À quoi bon? Et pourquoi souffrir autant? Elle dit qu'elle lave ses entrailles, qu'elle les vide de leur sang. Mais comment peut-elle extraire de ces viscères sanglants leur nature même?

(Elle s'approche un peu, prêtant l'oreille)

Elle dit qu'elle se lave, et même s'il n'est pas évident que ces choses-là se lavent, elle les lave quand même.

(Elle s'adresse de plus près à la bonzesse qui tient ses intestins entre ses doigts fins et les nettoie, morceau par morceau, avec habileté)

Pourquoi s'en donner la peine si c'est en vain? Continue-t-elle ainsi? Et quand cela prendra-t-il fin ${ }^{18}$ ?

ELLE ne représente plus l'unité de sa vision et de son corps, mais devient comme un pur regard: la perception se montre devant ELLE par le truchement de la bonzesse. Grâce à cette rupture, ELLE

18. GAO, X. J., Théâtre 1, op. cit., p. 80 
parvient à s'observer elle-même comme un Autre — ou bien à observer un autre comme elle-même. L'invisible qui est conçu dans le corps visible se fait voir lorsqu'ELLE ne raccorde plus son champ visuel, et lorsque ce corps possède sa propre subjectivité qui n'est plus à ELLE. Le corps est ainsi à la fois le sujet et l'objet du voir.

Si le lavage des entrailles de la nonne reflète l'ambition politique du général comme dans un miroir dans La Montagne de l'âme, il expose dans $\mathrm{Au}$ bord de la vie le désir sexuel mais aussi la sensation repentante ${ }^{19}$. Pour ainsi dire, la façon de voir son propre désir par le reflet du corps de l'autre se change en une sorte d'assimilation: la transposition de la sensation de la bonzesse à ELLE qui a lieu lorsqu'ELLE fixe le regard sur la bonzesse est rendue par l'utilisation subtile des pronoms personnels. ELLE parle tout le temps en forme de " ELLE dit qu'ELLE... », de " ELLE sait qu'ELLE » ou de « ELLE voit... ", de « ELLE dit... » pour exprimer la sensation qui vient toute seule en ELLE; mais pendant qu'ELLE contemple la bonzesse, sa parole commence par "Elle dit qu'elle »: " À quoi bon? Et pourquoi souffrir autant? Elle dit qu'elle lave ses entrailles, qu'elle les vide de leur sang. Mais comment peut-elle extraire de ces viscères sanglants leur nature même? " Il s'agit sans doute, avec cet « elle », du corps: celui de la bonzesse mais aussi celui d'ELLE-même. Cet elle et non pas ELLE qui devient à la fois le sujet voyant, le sujet percevant et le sujet de parole révèle justement une transmission directe entre les deux corps qui produit en effet une "métathèse » d'ELLE et d'elle. C'est que la vision qui se détache d'ELLE fait voir à celle-ci l'invisible de son corps qui se détache aussi d'ELLE, et réunit finalement les deux. En d'autres termes, la sensation du corps désirant qui revient en ELLE en assimilant la sensation de la bonzesse, n'est plus la sensation d'un donné invisible mais celle d’un donné partagé visible et épouvantable.

\section{Miroir transparent de l'intérieur}

De la réversibilité du général et de la nonne à celle qu'on constate entre ELLE et la bonzesse, il semble que Gao change le miroir réfléchi

19. Selon Gao, dans une conversation entre lui et nous-même le 10 févier 2013, l'autodissection est un symbole du complexe particulier à l'égard de la chair qu'entretiennent les femmes (surtout les femmes chinoises) : un mélange de désir et de regret. 
extérieur en miroir transparent de l'intérieur: car, comparé au corps de la nonne qui constitue un miroir - ce qui fait que le général y voit son propre corps -, le corps de la bonzesse n'est plus le support réfléchissant une image, mais le point d'attache et de rebond d'une perception intérieure à Elle qui s'étend jusqu'en dehors d'elle-même.

Il convient de remonter d'abord à la conception du miroir chez Gao; elle traduit un regard particulier sur le moi: que reflète-t-il de lui-même sur le miroir? que voit-on à travers le reflet? qui/que représente l'image dans le miroir? Dans La Montagne de l'âme, le chapitre 26 est consacré à une quête du moi par le truchement de la vision dans « le miroir »:

Je ne sais pas si tu as déjà réfléchi à cette chose étrange qu’est le moi.

[...]

Un jour, j’ai observé la photo collée sur ma carte d'autobus posée sur la table. Dans un premier temps, j'ai trouvé mon petit sourire plutôt agréable, mais ensuite, je l'ai trouvé plutôt railleur, un peu hautain et froid, témoignant d'un certain amour-propre mêlé de pas mal d'autosatisfaction, il indiquait que je me prenais pour un personnage supérieur. En réalité, j'y ai perçu une sorte d'affectation accompagnée d'une expression de grande solitude et de frayeur diffuse; ce n'était pas du tout le visage d'un gagnant. On y lisait de l'amertume. Il ne pouvait bien sûr pas y avoir le vague sourire habituel qui naît du bonheur involontaire, c'était plutôt une expression de doute quant au bonheur. Cela devenait un peu effrayant et même vain. La sensation de tomber sans point de chute. Je n'ai plus voulu revoir cette photo.

Ensuite, j'ai observé les autres, mais quand je l'ai fait, j'ai découvert que ce moi détestable et omniprésent s'en mêlait aussi, ne tolérant pas de ne pas intervenir dans la perception du visage de l'autre. C'était fâcheux: lorsque j'observais une autre personne, je continuais à m'observer moi-même. Je recherchais des visages que j'aimais, ou une expression que je pouvais accepter. Si un visage n’arrivait pas à me toucher, si je n'arrivais pas à trouver des gens avec qui m'identifier parmi ceux qui passaient devant moi, je les observais donc sans les voir [...] Quand j'observe les autres je les considère comme des miroirs qui me renvoient ma propre image et cette observation dépend entièrement de ma disposition d'esprit du moment. Même lorsque je regarde une jeune fille, je cherche à l'appréhender avec mes propres sens [...] Dans mon regard, les femmes ne sont rien d'autre que des illusions que j'ai créées moi-même et que j'utilise pour me mystifier. Voilà ce qui m'attriste ${ }^{20}$.

20. GAO, X. J., La Montagne de l'âme, op. cit., p. 212-213. 
" Je " prend sa propre photo et les visages d'autres personnes comme le miroir dans lequel il cherche son image. Mais il y a de l'absence dans la vision de « je »: lorsque « je " regarde sa photo, il voit un moi défamiliarisé qui lui paraît un inconnu; lorsqu'il regarde autrui, il ne voit qu'un moi arbitraire qui se présente partout. Quant aux objets authentiques qu'il tente d'observer, ils échappent à ses yeux. Même si la photo de "je » et les visages des autres ne constituent qu'un miroir imaginaire; la confusion ou le fourvoiement devant cette image reflétée dans le miroir n'est pas un hasard. Dans une autre pièce en un acte, Imitateur ( “模仿者”) écrite en 1983, le protagoniste principal, un peintre, passant devant le miroir, reste fasciné par l'image qui s'y donne à voir, puis commence à lui parler, à discuter et finalement à se disputer avec elle sans savoir que c'est le reflet de lui-même.

Il semble que Gao doute de la crédibilité du "voir son image » dans le miroir : aucun lien cohérent ne peut être établi à travers un miroir, imaginaire ou réel, ni entre image et sujet voyant, ni entre image et objet visible.

Merleau-Ponty explique le fonctionnement du miroir en y assimilant la réflexivité du corps: la chair est phénomène de miroir et le miroir est extension de mon rapport à mon corps ${ }^{21}$.

Le miroir apparaît parce que je suis voyant - visible, parce qu'il y a une réflexivité du sensible, il la traduit et la redouble. Par lui, mon dehors se complète, tout ce que j'ai de plus secret passe dans ce visage, cet être plat et fermé que déjà me faisait soupçonner mon reflet dans l'eau. Schilder observe que, fumant la pipe devant le miroir, je sens la surface lisse et brûlante du bois non seulement là où sont mes doigts, mais aussi dans ces doigts glorieux, ces doigts seulement visibles qui sont au fond du miroir. Le fantôme du miroir traîne dehors ma chair, et du même coup tout l'invisible de mon corps peut investir les autres corps que je vois ${ }^{22}$.

Ainsi le miroir ne reflète pas seulement notre image, mais aussi figure et amplifie la structure métaphysique de notre chair:

Désormais mon corps peut comporter des segments prélevés sur celui des autres comme ma substance passe en eux, l'homme est miroir

21. Merleau-Ponty, M., Le Visible et l'Invisible, suivi de Notes de travail, op. cit., p. 309.

22. Merleau-Ponty, M., L'ÉEil et l'Esprit, dans Maurice Merleau-Ponty, Euvres, op. cit., p. 1601. 
pour l'homme. Quant au miroir il est l'instrument d'une universelle magie qui change les choses en spectacles, les spectacles en choses, moi en autrui et autrui en $\mathrm{moi}^{23}$.

Lorsque Merleau-Ponty dit que le corps est une chair réflexible (touchant-touchable, voyant-visible...), la notion de l'Autre est déjà impliquée dans sa théorie. Il y a aussi une analogie qui préexiste entre moi et l'autre de même qu'entre le miroir et moi: c'est pourquoi par l'échange des regards de l'un à l'autre, moi se fait voir. MerleauPonty cite Paul Valéry pour avancer que «l'homme est miroir pour l'homme »: " Personne ne pourrait penser librement si ses yeux ne pouvaient quitter d'autres yeux qui les suivraient [...] Ce qui me manque, c'est ce moi que tu vois. Et à toi, ce qui manque, c'est toi que je vois. Et si avant que nous allions dans la connaissance l'un de l'autre, autant nous nous réfléchissons, autant nous serons autres [...] Je te vois, pour n'être pas toi, n'étant pas $\mathrm{Toi}^{24}$. " Sans te regarder, je ne sais pas ce qui me manque pour être moi-même, mais te regarder, c'est pour bien être moi-même, et réciproquement. En d'autres termes, le regard de l'autre anime le regard du moi sur moi-même.

Bien que la réflexivité sensible, comme extension de la réversibilité du corps, se présente partout chez Gao sous les figures du sujet percevant-perceptible, voyant-visible, particulièrement dans la narratologie des pronoms personnels, elle ne suppose pas vraiment une notion de l'Autre par laquelle l'échange entre moi et l'autre serait effectué. Même si le regard de la fille réveille Marguerite, il ne s’agit pas d'un regard-échange: la fille est l'Autre qui est révélateur pour Marguerite, mais Marguerite n'est pas un Autre pour la fille. D'autant plus que les personnages des pronoms personnels ne sont que les autres moi. Dans les ouvrages de Gao, il y a une tendance à réduire l'autre: soit l'autre est intériorisé par moi comme une projection du moi, telles les figures masculines pour ELLE dans $A u$ bord de la vie; soit le moi se divise comme les autres moi ou comme un moi qui est l'autre, tel que « tu » et « elle » pour « je » dans La

23. Ibid.

24. VALÉRY, P., «Aphorisme», dans La Nouvelle Revue française, septembre 1930, n²04, p. 296. La citation d'ici n'est pas tout à fait la même que celle que Merleau-Ponty fait dans « L'homme et l'adversité », dans Maurice Merleau-Ponty, Euvres, op, cit., 2000, p. 1385. 
Montagne de l'âme et « il " pour " tu » dans Le Livre d'un homme seul, ou le parleur B pour le parleur A dans Le Quêteur de la mort.

Il y a aussi un cercle du regard dans la contemplation du moi: chez Paul Valéry et chez Merleau-Ponty, le regard qui est basé sur l'analogie (plutôt métaphysique) est reflété par la réflexivité du corps entre moi et l'autre; ce cercle s'établit chez Gao à l'intérieur du moi ou bien entre les moi. Les " autres " ne se servent pas d'un miroir réfléchi à travers lequel "je " se voit lui-même. Prenons encore l'exemple d'Au bord de la vie: l'œil qui fait qu'ELLE se voit elle-même dans sa propre vision compose avec ELLE un cercle - remarquable - de la vue:

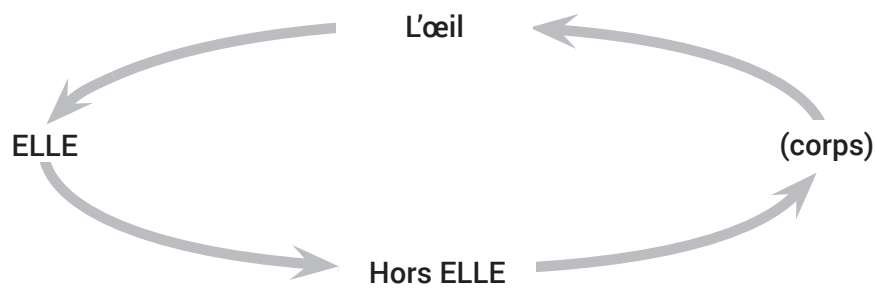

L'œil qui est ailleurs qu'ELLE - celui d'ELLE mais hors d'ELLE constitue un miroir de l'intérieur transparent, réalise un cercle entre ELLE et son corps. Lorsqu'ELLE voit son propre corps, celui-ci fait qu'ELLE éprouve aussi une perception visuelle à travers cet œil en dehors:

ELLE se voit maintenant, allongée dans les ténèbres, onduler légèrement et glisser sans poids; ELLE voit son propre corps, nu, sur le point d'être immergé, à peine porté par la crête des vagues noires, mais invisibles, puis plonger profondément. Quand une nouvelle vague montante la soulève, ELLE se livre aussitôt à la chute suivante, plus profonde encore, et plus noire ${ }^{25}$.

C'est pourquoi le chapitre 58 , qui semble répondre au doute de « je » dans le chapitre 26, propose une vision intériorisée et invite à entrer dans le miroir afin de voir le vrai moi:

Ce « moi » au milieu de « tu » n'est qu'un reflet dans le miroir, l'image inversée des fleurs dans l'eau; si tu n'entres pas dans le miroir, tu

25. GAO, X. J., Théâtre 1, op. cit., p. 82-83. 
n'arriveras pas à repêcher quoi que ce soit et tu ne feras que t'apitoyer sur toi-même en pure perte ${ }^{26}$.

À l'opposé de la vision qui forme l'image de soi dans l'eau/miroir extérieur, la vision intériorisée révèle certainement l'influence de la pensée du bouddhisme chán chez Gao. Ainsi à la fin du chapitre 26, « je » conclut en citant la parole du Bouddha:

Voilà pourquoi Bouddha a enseigné l'éveil: toutes les images sont des vides et des illusions, la non image est aussi le vide et l'illusion ${ }^{27}$ (万相皆虚妄, 无相也虚妄).

Il y a une histoire chán (gong’an, 公案) entre Pei Xiu et Huang $\mathrm{Bo}^{28}$ qui éclarcit le concept du moi dans le bouddhisme chán. Pei Xiu (791-864), gouverneur et calligraphe de la dynastie Tang, visitait un jour un monastère placé sous sa juridiction. Il arriva dans une salle où se trouvaient exposés les portraits des différents abbés. Pei désigna l'un d'entre eux et demande à l'abbé: « Qui est celui-là? » L’abbé répondit: "Feu notre dernier abbé. » Question suivante de Pei: "Je vois bien son portrait, mais où est la personne? "C'était plus que ne pouvait répondre l'abbé. Celui-ci en appela au maître Huang Bo qui faisait un séjour dans ce temple. Pei répéta ainsi sa question: "Je vois bien ici le portrait du défunt abbé, mais la personne, où estelle? " Aussitôt le maître appela à voix haute: " Ho! Gouverneur! " Pei Xiu répondit: " Oui, Maître! " "J'entends le son du gouverneur, mais où est-elle, la personne? " lui demanda Huang Bo, à son tour, lui posant ainsi la question ${ }^{29}$.

Les questions de Pei Xiu et de Huang Bo demandent toutes « où » se trouve le soi authentique qui n’est représenté ni par l'image ni

26. GAO, X. J., La Montagne de l'âme, op. cit., p. 469.

27. Nous modifions la traduction de «toutes les images sont des mensonges, l'absence d'image est aussi mensonge », car l'absence d'image veut dire qu'il y avait une image mais qu'elle n'est pas là pour ce moment, voir GAO, X. J., La Montagne de l'âme, op. cit., p. 214.

28. Pei Xiu (裴休791-864), gouverneur et calligraphe de la dynastie Tang ; Huang Bo (黄藮/ 黄藮希运, ?-850) fut un grand maître de l'école chán de la dynastie Tang.

29. C'est une histoire issue de 《筠州黄藮山断际禅师传法心要》, Taibei, Guoli shifan daxue chubanshe (国立师范大学出版社), 2012. Daisetsu Teitaro Suzuki mentionne aussi cette histoire dans Bouddhisme Zen et psychanalyse (SUZUKI, D.T., FROMM, E. et DE MARTINO, R., traduit par Théo Léger, Paris, Puf (Quadrige), 1971, p. 32-33) ; ce que Suzuki raconte diffère par de petits détails de l'histoire originelle : nous la traduisons en comparant la traduction de Suzuki et la version originale. 
par la parole de l'extérieur, car le soi ne peut se connaître lui-même que de l'intérieur et non pas de l'extérieur.

Le soi dans la pensée du bouddhisme chán correspond à une découverte de la vacuité. La vision vers l'extérieur et la vision vers l'intérieur se distinguent juste pour cette école comme la manière fausse et la manière vraie de voir un soi authentique et vide - qui ne peut être ni peint, ni expliqué.

Ainsi, lorsque Gao s'interroge sur l'image dans le miroir/eau dans La Montagne de l'âme, et propose d'entrer dans le miroir, il change en fait la vision de l'extérieur vers l'intérieur en vision de l'intérieur vers l'intérieur, et convertit le miroir de l'extérieur en miroir transparent de l'intérieur, en se fondant sur un moi qui emprunte la notion du soi à chán, qui est pure et transparente, indépendante de son propre corps, du monde et de l'autre.

\section{UNE AUTRE MANIÈRE DE REGARDER LE MOI EN RAPPORT AVEC LA PENSÉE DU BOUDDHISME CHÁN}

\section{Le miroir est non-miroir}

On l'aura compris, comment voir le moi est une question essentielle chez Gao, et ce au niveau esthétique aussi bien que du point de vue philosophique. Il ne s’agit pas seulement de choisir une façon de fixer le regard sur le moi, mais avant tout, de savoir ce qu'est le moi. Pour ainsi dire, la notion du moi prédétermine, dans une large mesure, la manière de s'examiner.

La notion du moi chez Gao est fortement inspirée par la pensée du bouddhisme chán, pour lequel le moi ne saurait être représenté ni par l'image, ni par le langage. Depuis la fin des années 1980, cette conception du moi comme inexplicable est fréquente dans les œuvres de Gao, particulièrement dans La Montagne de l'âme, $\mathrm{Au}$ bord de la vie, Dialoguer-interloquer et La Neige en août. Le moi a perdu toute évidence et ne s’incarne plus dans une figure donnée; sa quête ne relève plus d'une démarche ouverte, patente: elle s'opère latente comme les veines cachées sous la peau.

Dans La Montagne de l'âme, Au bord de la vie et Dialoguer-interloquer notamment, la quête du moi est la véritable intrigue, latente 
mais ininterrompue; c'est sa prééminence qui amène à se poser des questions sur le rôle réel du narrateur, qui déroule des histoires sans véritable argument selon les critères de la narration classique. La Neige en août, qui parle de la vie du moine Huineng, semble interpréter cette grande figure chán par référence à la notion du moi travaillée dans l'intimité textuelle de ces trois ouvrages et virtuellement établie par eux.

Gao dédie en effet son premier (et dernier) opéra La Neige en août à Huineng, personnalité idéale à ses yeux. Il y a une scène remarquable où Huineng crée un poème chán (Ji, 偈) lors d'un concours établi pour choisir le successeur du temple où il est en rivalité avec un autre moine, Shenxiu; lorsque Huineng entend réciter le poème de ce dernier écrit sur le mur du temple, il demande à un peintre de tracer son propre poème sur le mur afin d'exprimer à son tour sa pensée, en se différenciant de Shenxiu à propos de la nature de Bouddha.

Shenxiu:

Le corps est l'arbre de l'Éveil

身是菩提树

L'esprit est comme le miroir clair ${ }^{30}$

心如明镜台

Appliquer sans cesse à l'essuyer et à le frotter

Pour ne pas avoir poussière

时时勤拂拭

莫使惹尘埃

Huineng:

L'Éveil ne comporte point d'arbre

菩提本无树

Ni le miroir clair de miroir ${ }^{31}$

La nature de Buddha est toujours pure et calme

Où y aurait-il de la poussière

明镜亦无台

佛性常清静

何处惹尘埃 ${ }^{32}$

30. 明镜台 (Mingjing-Tai), traduit mot à mot: 《la monture du miroir ». Ici, Tai (台) ne signifie plus la monture, mais le miroir lui-même. Car dans la langue chinoise, il y a des répétitions du même sens en différents mots.

31. Pour la même raison que celle évoquée ci-dessus, nous traduisons 明镜亦无台 par «le miroir clair ne comporte pas de miroir » au lieu de traduire littéralement: « le miroir ne comporte pas de monture ».

32. Voir l'opéra 《八月雪》 (La Neige en août), texte écrit en chinois et mis en scène par Gao, composé avec la musique de Xu Shuya, dont la première représentation a eu lieu à Taipei en 2002, puis à Marseille un an plus tard. II y avait plusieurs versions de ce poème de Huineng. Pour le deuxième vers : 《明镜亦无台》 ou 《明镜亦非台》, et pour le troisième : 《佛性常清静》 ou 《本来无一物》. Celle que Gao a retenue est sans doute la version originelle, celle de l'époque Tang que l'on trouve à Dunhuang (敦煌). Nous retraduisons les poèmes de Senxiu et de Huineng à partir de la traduction de Paul Demiéville, « Le Miroir spirituel », dans Choix d'études bouddhiques (1929-1970), Leiden, Brill, 1973, p. 112.-137. 
Cette scène se fonde sur l'histoire véridique de Huineng et de Shenxiu, qui sont les deux futurs fondateurs des écoles bouddhistes du Nord et du Sud pendant la dynastie Tang. Paul Demiéville traduit respectivement les deux écoles par " gradualisme " et " subitisme $»^{33}$. Leur différence principale ne porte pas seulement sur la façon de pratiquer le bouddhisme - éveiller graduellement ou subitement - mais aussi sur le concept de la nature de bouddha quand il s'agit de la capacité d'avoir la nature pure et innée chez tous les êtres humains d'un devenir bouddha - autrement dit, de la possibilité d'atteindre la nature du propre/soi authentique (本性). Lorsque Shenxiu, qui prend l'arbre d'Éveil sous lequel Bouddha atteignit la Bodhi pour la métaphore du corps, et le miroir qui reflète le monde pour la métaphore de l'esprit, insiste sur la nécessité de nettoyer les poussières afin d'avoir la pureté de la nature, il accorde une sorte de substantialité à cette nature et indique que la manière de devenir bouddha est un mouvement de l'extérieur vers l'intérieur. Paul Demiéville remarque: «La distinction de la pureté et de l'impureté implique déjà, en effet, un dualisme, un relativisme contraires à l'imprédicabilité de l'absolu, qui est "vide" de toute détermination ${ }^{34}$. "

Cet "imprédicabilité de l'absolu " dont parle Demiéville est justement ce que Huinneg illustre dans son poème: la nature de bouddha, c'est-à-dire la nature du propre soi ne se représente par aucune forme de l'existence. Huineng souligne l'insubstantialité du corps et de l'esprit, en montrant par une image inattendue que l'arbre de l'Éveil est sans arbre aussi bien que le miroir est sans miroir. De même, le corps est sans corps, et l'esprit (心) ne fonctionne pas non plus comme un miroir qui reflèterait le monde en donnant une vision. Le soi authentique est en soi, inutile de le chercher ailleurs.

Se différenciant radicalement de la pensée du moi qui repose sur l'être et qui cherche " qui je suis " et " de quoi je procède en tant

33. Demiéville avoue que les termes 顿悟 et 渐悟 sont difficiles à traduire: « [les termes] ne rendent que de manière trop littérale une opposition sémantique complexe que le français ignore; mais perfectivisme et imperfectivisme, totalitarisme et évolutionnisme, ne vaudraient guère mieux, et je m'en tiendrai provisoirement à subitisme et gradualisme ", dans « Le Miroir spirituel », op. cit., p. 117.

34. Ibid. 
que singularité du moi », la pensée du bouddhisme chán sur le soi ne cherche pas à se construire: elle s'établit sur le mode " $\mathrm{A}$ est non-A ". Car, si l'hypothèse " $A$ est égal à $B$ " est validée, l'image $B$ s'identifie à l'image A, les deux seront des représentations de A - il y aura deux images pour A: alors, laquelle est la véritable? Les deux images ne mèneront qu'à la confusion à l'égard de la nature authentique (tathata en sanskrit, zhenru en chinois: 真如) qui, selon le bouddhisme, n'a pas d'image, puisque l'image extérieure ne nous donne que l'illusion et nous conduit dans le piège de la "dualité " qui est incompatible avec le principe de non-deux (不二法门) $)^{35}$. Ainsi, dans l'un des plus essentiels sûtra du bouddhisme chán, Lankāvatāra (楞伽经), Bouddha réveille les gens: "De même que l'image qui est reflétée dans le miroir, visible mais irréelle, l'esprit reflété dans le miroir de sa propre imprégnation est vu par l'ignorant comme s'il y en a "deux" "36."

Certes, ce que la nature de tathata dépasse, ce n'est pas seulement les images A et B qui représentent A, mais encore A même: elle repose sur une vacuité pure, rien ne peut la représenter. Cette "logique " chán se reconnaît très fréquemment chez Gao: je ne

35. Huineng donne l'exemple de la nature de Bouddha pour expliquer le principe de non-deux: «la nature de Bouddha n'est ni constante ni non-constante, ainsi elle est ininterrompue, on l'appelle non-deux. (佛性非常非无常, 是故不断, 名为不二). Voir 《六祖坛经・行由品第一》(Sutra de l'Estrade, le premier chapitre), 《坛经校释 》 Tanjing jiaoshi, 北京 Beijing, 中华书局 Zhonghua shuju, 1983; voir aussi 坛经金陵刻经处 本。不二法门 est apparu comme un concept figuré dans les pièces de théâtre de Gao, notamment à la fin de Dialoguer-interloquer lorsque l'homme cherche une porte.

36. Demiéville les a cités dans son article «Le Miroir spirituel»; sa traduction était réalisée à partir de la version de Nanjio (Kyoto, 1923); nous prenons la version de Gunabhadra (求那跋陀罗) au $v^{e}$ siècle qui fut traduite du sanscrit au chinois, en nous référant à la traduction de Demiéville. Voici la traduction de Demiéville:

" De même que l'image irréelle

Est reflétée dans le miroir

L'esprit un, reflété dans le miroir de sa propre imprégnation

Est vu par l'ignorant comme une dualité. »

Voici la version chinoise de Gunabhadra

《譬如镜中像，虽现而非有

于妄想心镜, 愚夫见有二»

Voir 《楞伽阿跋多罗宝经》, dans 《禅宗七经》, 宗教文化出版社, 1997, p. 116. La raison pour laquelle nous ne traduisons pas 二 par dualité, c'est que 二 (deux) a le sens plus large que la dualité : il s'agit de tout ce qui est non un (非一). 
suis pas plus véritable que mon image, mais, en même temps, mon image ne me représente pas. Dans La Montagne de l'âme, " je " qui recherche la vérité dans le monde réel ne représente pas un moi plus authentique que son ombre « tu " qui est en quête de la montagne de l'âme dans le monde intérieur. De même, la montagne de l'âme n'est pas plus fictive que la vérité: toutes les deux ne représentent que deux noms aussi bien que deux images imaginaires d'une même chose - le " vrai » est en effet un non-vrai. C'est pourquoi Gao cite la parole de Bouddha comme la conclusion de ce que « je » cherche dans l'image de soi et des autres: "Toutes les images sont des vides et des illusions, la non image est aussi le vide et l'illu$\operatorname{sion}^{37}$ (万相皆虚妄, 无相也虚妄).”

Dans Dialoguer-interloquer, le même doute sur ce qu'est le moi est provoqué par le regard sur l'autre, puis sur soi-même:

La femme [...] Elle finit par voir quelqu'un de face, un homme dont elle espère qu'il pourra la secourir! De tout cœur, elle voudrait voir son visage, mais c'est la confusion, elle ne peut le distinguer. (Elle tourne autour de lui, puis l'examine) C'est une ombre!

(Déçue, la femme recule pas à pas, la tête baissée)

[...]

La femme (elle s'examine des pieds à la tête): Elle se demande si elle n'est pas une ombre, elle aussi. (Elle regarde l'ombre à ses pieds, et tourne sur elle-même) Ou cette ombre, c'est elle? (Elle est prise de vertige) Ou bien elle n'est que l'ombre de cette ombre? (Elle ferme les yeux) Qui est-elle au juste ${ }^{38}$ ?

La femme cherche l'image de l'homme, qui ne lui paraît qu'une ombre, puis elle sombre dans la confusion entre elle-même et sa propre ombre. L'ombre - notion décidément essentielle chez Gao — révèle l'insubstantialité substantielle de la femme: il ne s'agit pas d'une négation du moi, mais plutôt d'un non-moi. Pour ainsi dire, pour voir le vrai moi, il faut viser un non-moi qui n’est ni le néant ni la substantialité - comme la métaphore du « non-miroir du miroir ", mais est là en tant que nature authentique (tathata).

Comment montrer ce non-moi?

37. GAO, X. J., La Montagne de l'âme, op. cit., p. 214; nous modifions la traduction.

38. GAO, X. J., Dialoguer-interloquer, traduction d'Annie Curien, Belgique, Lansman, 2001, p. 59. 


\section{Se perdre pour atteindre le vrai moi}

Dans La Neige en août, il y a ce dialogue entre le maître Hongren et Huineng, lorsque le premier veut transmettre au second la robe de cérémonie et le bol qui sont les symboles de la légitimité:

Hongren. En deçà de la porte, qu’y a-t-il (门里有什么? )

Huineng. Le maître et moi. (和尚和我)

Hongren (il rit). Et quelle chose est ce moi? (我为何物?)

Huineng. Une pensée intérieure. (心中之念)

Hongren. Où se trouve-t-elle? (何处? )

Huineng. La pensée n'est jamais interrompue, elle est partout. (念念不断, 无所不有).

Honren (il s'exclame). Si elle n'a aucun endroit où s'arrêter, pourquoi t’y accroches-tu encore? (无所住, 还念个什么? )

Huineng (silencieux, il baisse la tête. Un instant plus tard, il relève la tête). Pour rien. (没了)

Hongren. Alors, pourquoi dire qu'elle existe? (又何以说有? )

Huineng. Uniquement parce que, à l'instant, le maître a posé la question... (只因和尚刚才问......)

Hongren. Il n’y a pas d'“ à l'instant »! (无有刚才！ ) 39 $^{39}$

Dans le noir, le son d'un lourd coup de tambour. Hongren se retourne, il s'empare d'un bâton près du lit de méditation pour tracer un cercle sur le sol.

Huineng (il se penche pour regarder le cercle et relève la tête). Le vide. (空的)

Nouveau coup de tambour. Hongren brandit son bâton et décrit un nouveau cercle autour de lui.

Huineng lève la tête et regarde Hongren en riant. Nouveau coup de tambour.

Hongren (il rit à gorge déployée). Sous les mots et ta nature originelle que tu as reconnue par toi-même, voilà un homme authentique, un maître, un Bouddha! (言下自识本性，即丈夫、天人师、佛！) La grande intelligence mène à l'autre rive ${ }^{40}$ ! (大智慧到彼岸是 也! )

Hongren révèle à son futur successeur que le soi authentique ne peut être expliqué par le langage, comme tout ce qui ne peut être vu par l'image. La vacuité qui fait venir la grande intelligence

39. Nous modifions la traduction de Noël Dutrait: «À l'instant j'ai ne rien demandé! ». 40. Nous prenons la traduction de Noël Dutrait, mais mettons aussi à côté le texte chinois mis entre parenthèses qui est presque intraduisible. Voir La Neige en août, Seuil, Paris, 2004, p. 149-151; voir aussi 《八月雪》, 联经出版社,台北, 2000, p. 28-29. 
(大智慧) émerge toute seule lorsqu'on n'en parle plus. C'est aussi l'anticipation de chán: "Ne pas reposer sur l'écriture, mais toucher directement l'esprit: devenir le bouddha dès que l'on voit la nature de tathata (不立文字, 直指人心, 见性成佛)». Au sens littéral, le mot français « intelligence " est issu du latin intelligentia, de la famille de legere (lire) qui signifie " recueillir par les sens (oreilles, yeux) ». Mais avec 大智慧 (sagesse) en chinois, notamment dans le bouddhisme chán, il s'agit plutôt d'une " a-intelligence " qui ne se rattache pas à la connaissance. Rappelons que Huineng, sixième maître de l'école chán, ne savait ni lire ni écrire. D’ailleurs, en tant que bouddhisme indien taoïsisé, le bouddhisme chán partage le point de vue de Zhuangzi quant à la capacité limitée du langage, car le langage qui prétend éclairer les choses ignore pourtant l'ombre de soi-même: «Dans tout débat, il y a de l'indiscuté [...] dans tout débat, il y a du non-vu (辩也者, 有不辩也 $[\cdots]$ 辩也者, 有不见也 ${ }^{41}$ ). ”

En effet, "la nature de bouddha " dans le bouddhisme chán et "l'homme authentique " chez Zhangzi ne sont que deux interprétations similaires d'un vrai moi qui se fonde sur le "vide " (空) et sur le « il n’y a pas » (无): ils sont homogènes de Dao (道). Il y a un dialogue de Zhuangzi où Zikui qui vient de Nanbo demande à Nüyu où elle a eu le Dao. Nüyu dit:

J'en ai entendu parler du fils d'Encres, qui en a entendu parler du petit-fils de Récitation, qui en a entendu parler de Vision lumineuse, qui en a entendu parler de Chuchotement, qui en a entendu parler de Service nécessaire, qui en a entendu parler de Litanie bredouille, qui en a entendu parler d'Obscurité profonde, qui en a entendu parler de Vide immense, qui en a entendu parler d'Origine incertaine ${ }^{42}$.

Cette origine incertaine, le Dao, n'est pas éprouvée directement par le langage (Encres, Récitation) ni par la vision: c'est le vide et l'obscurité qui transmettent le Dao. Il s'agit d'une sorte d'a-vision et d'a-langage qui s'identifient à la " grande sagesse " pour atteindre le soi authentique.

41. ZHUANGZi, «庄子集释・齐物论》(Zhuangzi Jishi), op. cit., 2004, p. 83.

42. ZHUANGZI, «庄子集释 - 大宗师» (Zhuangzi Jishi), op. cit., p. 256 ; nous traduisons du chinois “闻诸副墨之子, 副墨之子闻诸洛诵之孙, 洛诵之孙闻之瞻明, 瞻明闻之 聂许, 聂许闻之需役, 需役闻之于讴, 于讴闻之玄冥, 玄冥闻之参寥, 参塞闻 之疑始”。 
Chez Gao, l'a-vision et l'a-langage paraissent la façon de se perdre. La poursuite du moi, dans La Montagne de l'âme, semble justement une interprétation romanesque de cette pensée du bouddhisme chán et de Zhuangzi: le moi ne peut être vu par l'image et ne peut non plus être expliqué par le langage, il est réveillé lorsqu'il se perd dans différents endroits.

Dans ce roman, la recherche du moi s'incarne respectivement dans la recherche de la montagne de l'âme, déployée par le dialogue de «tu " et « elle " à travers les contes, les folklores et les histoires lointaines sur l'axe du temps, et dans la recherche de la vérité déroulée par l'histoire de " je » à travers son voyage sur l'axe de l'espace. Cependant, la montagne de l'âme est introuvable, tout comme la vérité est indéfinissable à travers le langage et la vision. Le dialogue de " tu " et d'" elle " s'étiole après une longue et vaine quête de la montagne de l'âme. À partir du chapitre 52, "elle " a disparu et est remplacée graduellement par les autres femmes sous le même pronom personnel " elle »; la description de la perception de « tu » remplace aussi le récit dialogique. Parallèlement, « je » ne tient plus à chercher le sens de ce qu'il voit. Le chapitre 26 est comme une prémonition de ce renoncement. Puis dans le chapitre 77, « je » montre une image faisant " non-sens » de la neige:

Le sens de ce reflet n'est pas clair [...] Le ciel sombre, le sol couvert de neige plus brillant encore par contraste avec ce ciel sombre, plus de merles, plus de moineaux, la neige a absorbé toute idée et tout sens ${ }^{43}$.

Lorsque « tu » et « je " renoncent à chercher le sens dans le langage et dans la vision, ils se rapprochent l'un de l'autre et de plus en plus. À partir du chapitre 62, «il " qui est une autre silhouette du moi se présente et remplace petit à petit « tu » dans les chapitres 62 , 72 et 76 . « $\mathrm{II}^{44}$ » apparaît d'emblée comme une symbolisation de la perte (ch. 62):

43. GAO, X. J., La Montagne de l'âme, op. cit., p. 635-639.

44. Gao explique l'apparence d'« il » : « Tu es dans ton propre voyage spirituel, tu erres dans le monde entier avec moi en suivant tes pensées, et plus tu vas loin, plus tu te rapproches jusqu'à ce que, inévitablement, il devienne impossible de nous dissocier; il te faut alors reculer d'un pas et cette distance qui se crée, c'est « lui », et « lui », c'est une silhouette lorsque tu me quittes et t'éloignes. » (La Montagne de l'âme, op. cit., p. 422). 
Tu dis qu'il a perdu la clef.

[...]

Il s'était perdu lui-même. Ses souvenirs lui échappaient. Il réfléchissait de toutes ses forces, tentant de se rappeler quelles relations il entretenait avec qui, pourquoi il était dans cette rue ${ }^{45}$. [...]

Puis dans le chapitre 76, " il " ne sait pas où il se trouve:

Après une longue errance, dans la solitude, il arrive face à un vieillard appuyé sur une canne, vêtu d'une longue robe. Alors, il lui demande conseil:

- S'il vous plaît, vieil Homme, où se trouve la Montagne de l'Âme?

- D’où viens-tu? rétorque le vieillard.

Il répond qu'il vient de Wuyi.

- Wuyi? Le vieillard réfléchit un instant. Ah oui, près du fleuve.

Il dit qu'il vient précisément du bord du fleuve, s'est-il trompé de chemin? Le vieillard fronce les sourcils:

- Le chemin est bon. C'est celui qui l'emprunte qui s'est trompé.

- Vous avez entièrement raison, Vieil Homme.

Mais il veut lui demander si la Montagne de l'Ame se trouve au bord du fleuve.

- Si l'on dit qu'elle est au bord du fleuve, c'est qu'elle est au bord du fleuve, répond le vieillard sur un ton impatient.

Il dit qu'il est justement venu de ce bord-là vers ce bord-ci.

- Plus tu marches plus tu t'éloignes, dit le vieillard, sûr de lui.

Bon, dois-je faire demi-tour? demande-t-il à nouveau.

En lui-même il se dit qu'il n'y comprend vraiment rien.

- Ce que j'ai dit est très clair, répond le vieillard froidement.

- Oui, c'est vrai, Vieil Homme, c'est très clair...

Le problème, c'est que lui-même n'y voit pas encore clair.

- Qu'est-ce qui n'est pas clair? demande le vieillard en le scrutant sous ses épais sourcils.

Il dit qu'il ne comprend toujours pas comment aller à la Montagne de l'Âme.

Les yeux fermés, le vieillard se concentre.

- N'avez-vous pas dit qu'elle était là-bas, au bord du fleuve?

Il repose la question.

- Mais je suis déjà allé là-bas...

- Oui, elle est là-bas. Il l'interrompt, impatient.

- Et par rapport au bourg de Wuyi?

- Eh bien, elle est toujours là-bas, au bord du fleuve.

45. GAO, X. J., La Montagne de l'âme, op. cit., p. 519-525. 
- Mais c'est justement à Wuyi que j'ai rejoint l'autre rive du fleuve, quand vous dites là-bas, au bord du fleuve vous voulez dire en fait de ce côté-ci du fleuve!

- Vous ne voulez pas aller à la Montagne de l'Âme?

- Mais si.

- Eh bien, elle est là-bas, au bord du fleuve.

- Vieil homme, c'est de la métaphysique, c'est cela?

Il reprend sur un ton très sérieux:

- Vous ne m'avez pas demandé le chemin?

Il dit que si.

- Eh bien, je vous ai renseigné.

Appuyé sur sa canne, le vieillard s'éloigne, pas à pas, sans plus lui prêter attention.

Et il reste seul de ce côté-ci du fleuve, de l'autre côté par rapport à Wuyi. En fait, le problème est de savoir de quel côté est Wuyi. Il ne sait vraiment plus. Seule lui revient en mémoire une comptine vieille de plusieurs milliers d'années:

- Rentrera, rentrera pas, mais là ne reste pas. Au bord du fleuve le vent est froid ${ }^{46}$.

Le vieil homme qui accepte de renseigner « il ", s'emploie avec une apparence de brusquerie à le fourvoyer, ou plus exactement à lui répondre en ignorant le principe de contradiction qui fait qu'un chemin ne peut équivaloir, pour aller quelque part, à son opposé. Mais la confusion qui provient exactement de la distinction des choses, est la clef pour trouver la montagne de l'âme. Car, si l'on ne distingue plus ce côté-là et ce côté-ci du fleuve, s'il n’y a plus lieu de s'orienter, il n’y aura plus de perte d'orientation. La montagne de l'âme qui est la projection du vrai moi, introuvable en suivant l'une ou l'autre rive, ou quelque rive que ce soit, révèle par son absence physique que le vrai moi est en moi et qu'il est inutile de le chercher ailleurs.

Ainsi, à la fin du roman, lorsque « je » et " tu " ne se distinguent plus et abandonnent la recherche du moi, dans le monde intérieur comme dans le monde extérieur, et ne s'accrochent plus au langage et à la vision, mais en viennent à admettre la perte de soi et la non-distinction, le vrai moi apparaît de lui-même. Dans le dernier chapitre, « je » voit une grenouille qui lui parle avec un œil:

46. GAO, X. J., La Montagne de l'âme, op. cit., ch.76, p. 631-633. 
Par la fenêtre, je vois sur le sol enneigé une minuscule grenouille. Elle cligne un œil et écarquille l'autre. Elle m’observe sans bouger. Je comprends qu'il s'agit de Dieu.

Il se manifeste à moi sous cette forme et regarde si j'ai compris.

Il cligne de l'œil pour me parler. Quand Dieu parle aux hommes, il ne veut pas qu'ils entendent sa voix. Moi, cela ne m'étonne pas, comme s'il devait en être ainsi, comme si Dieu avait toujours été une grenouille avec un œil tout rond, intelligent, grand ouvert. Quelle miséricorde de sa part de bien vouloir s'occuper d'un homme aussi pitoyable que moi!

Le langage incompréhensible qu'il parle de son autre œil, en clignant la paupière à l'attention des hommes, il me faut le comprendre. Mais cela, ce n'est pas son affaire.

Je peux également estimer que ce clignement de paupière n'a aucun sens, mais son sens réside peut-être justement dans son absence de sens $^{47}$.

Aux yeux de « je », l'œil qui cligne de la grenouille révèle la vérité mais sans rien dire, tandis que l'autre œil rond semble l'observer mais sans rien signifier. Ce langage sans parole et ce regard parlant qui ne distinguent pas sens et non-sens éveillent pourtant la non-distinction dans « je » et dans ce qu'il voit: la grenouille/Dieu qu'il voit, est son propre moi qui est à la fois en moi et hors de moi, à la fois le moi et le non-moi.

\section{Je me perds dans la vision où je suis, je me " retrouve " dans la vision où je ne suis pas}

Il y a enfin un modèle dans la poursuite du moi chez Gao: chercher-se perdre-s’éveiller. La recherche du moi provoque, particulièrement dans les récits de " je », une série de moments de perte: perte dans la montagne ou dans l'obscurité de la forêt, enlisement dans les vases du lac. Pour en sortir, il ne s'agit pas de disposer d'une connaissance ou d'un savoir-faire; seule l'attitude consistant à laisser se faire ce qui nous arrive, éclaire le « je » dans sa situation de désorientation.

Tel le leitmotive dans la symphonie, ce mode récurrent du nonagir, de l'abandon apparaît sous plusieurs formes similaires dans $L a$ Montagne de l'âme, en faisant advenir une perception visuelle du

47. Ibid., p. 669. 
non-moi. Le chapitre 18 rapporte une situation difficile pour " je ", qui a voulu monter dans un petit bateau sur un lac mais s'enfonce dans la vase:

Je dégage mon pied pour avancer d'un pas, mais je m'enfonce jusqu'à mi-jambe dans la vase. Je n'ose pas faire porter mon poids vers l'avant. Je sais que dès que mon genou sera enfoui, je n'aurai plus aucun moyen de me sortir. Je n’ose pas non plus déplacer mon pied arrière. Incapable ni d'avancer ni de reculer, je ne sais plus quoi faire ${ }^{48}$.

Dans ce moment difficile où " je " ne peut avancer ni reculer, il perçoit cependant un silence solitaire - ce qu'il recherchait sans doute, se dit-il — reflet d'un monde ${ }^{49}$ (境界) intérieur qu'il ne peut éprouver dans les circonstances ordinaires:

À la surface silencieuse de l'eau, même les oiseaux aquatiques ont disparu. Imperceptiblement, les eaux brillantes commencent à s'obscurcir. À partir des touffes de roseaux, se répandent les couleurs du crépuscule, un air frais monte sous mes pieds. Je suis transi. Ni stridulations de grillons, ni coassements de grenouilles. Peut-être est-ce là enfin cette solitude originelle dénuée de sens que je recherchais ${ }^{50}$.

Cette réaction inattendue rappelle la façon de parvenir instantanément à l'illumination dans le bouddhisme chán. Dans la pensée chán, la situation de l'impasse est traitée comme révélateur d'illumination. Le maitre Huiqing (芭蕉慧清禅师) propose cette situation à ses élèves: «En route, un passager trouve soudainement devant lui un ravin de cent mille mètres de profondeur; derrière lui le feu l'attrapera; et des deux côtés, ce sont des broussailles d'épines. Il tombera dans le ravin s'il avance, il sera brûlé par le feu s'il recule; il se blessera dans les broussailles s'il se tourne vers la gauche ou la droite $^{51}$ ", pour leur montrer que la façon de se "sauver " soi-même

48. Ibid., p. 162.

49. 境界 (《monde ») est une notion esthétique qui occupe une place très particulière en littérature et pensée chinoises : nous en déploierons le sens dans le passage suivant.

50. GAO, X. J., La Montagne de l'âme, op. cit., p. 162.

51.PU, J. (普济), 《五灯会元》(WuDeng Huiyuan), 北京 Beijing, 中华书局 Zhonghua Shuju, 1984, p. 551. Nous traduisons du chinois «芭蕉慧清禅师: 如人行次, 忽遇前 面万丈深坑, 背后野火来逼, 两畔是荆棘从林, 若也向前, 则堕在坑堑。若也 退后, 则野火烧身。若也转侧, 则被荆棘林碍。当与么时, 作么生免得? 》 
n'est pas de chercher une solution envisageable dans une situation sans issue, mais de tout laisser et de s'en détacher. Su Shi, la grande figure littéraire du $\mathrm{XI}^{\mathrm{e}}$ siècle, donne un bon exemple. Il est à mi-chemin de sa promenade dans la montagne vers le kiosque Songfeng:

Fatigué de marcher, j'eus envie d'aller me reposer sous les arbres. Contemplant le kiosque, si haut perché, je me demandai comment j'arriverais jusqu'à lui. Longtemps j'ai réfléchi, puis me dis soudain: «Y a-t-il en ces parages un seul endroit où il ne serait pas délicieux de se reposer? " Et je me sentis heureux comme un poisson brusquement libéré de l'hameçon auquel il fut pris. Après cette illumination, quand bien même j'aurais été au cœur d'une bataille sans merci, sûr d'être tué par l'ennemi si j'avançais, ou de subir la peine de mort si je reculais, je n'en aurais pas moins pu goûter à tout instant le repos le plus parfait ${ }^{52}$.

La révélation de Su Shi est en quelque sorte le modèle de celle que " je " éprouve dans la vase: ce qui est en cause, ce n'est pas l'« ignorance » de la situation où l'on se trouve, mais l' "ignorance » de soi-même. Car la situation d'impasse est fondamentalement provoquée par la conscience de soi. Ainsi, dans La Montagne de l'âme, la désorientation n'est pas tant due à (une défaillance de) la vision qu'à la conscience incontrôlée du moi. À la fin du chapitre 65, cet égarement de « je » dans la montagne est causée par son imagination:

Je devais absolument me contrôler et quitter en toute hâte cette montagne. Sans m'occuper des buissons couvrant le sous-bois, je profitai d'un tournant du sentier afin de m'y précipiter et m'agripper à un tronc d'arbre pour freiner ma course. Mes mains et mon visage brûlaient, il me semblait que le sang coulait sur mes joues. Levant la tête, j'ai vu sur une branche un œil tout rond qui me fixait. Je regardai autour de moi, partout les branches ouvraient de grands yeux et m'observaient froidement.

Je devais me calmer, ce n'était après tout qu'une forêt d'arbres à laque. Les montagnards qui avaient récolté le laque avaient laissé des entailles sur les troncs des arbres. Ils poussaient dans cet état, créant ce paysage infernal. Je pourrais dire aussi qu'il ne s'agissait que d'une illusion due à ma peur intérieure; mon âme noire m'épiait, ces yeux multiples, c'était en fait moi-même qui m'observais. J'ai toujours eu l'impression d'être continuellement espionné, ce qui a sans

52. Su, S. (苏轼), 《记游松风亭 》 (《Du voyage du kiosque Songfeng »), dans 《苏轼文 集 » Sushi wenji, Beijing, Zhonghua shuju, 1986, p. 2271. 
cesse gêné mes mouvements. En réalité, il s’agit seulement de la peur que j'ai de moi-même ${ }^{53}$.

Les yeux sur les branches ne sont que les yeux extériorisés de " je ", autrement dit, ces yeux hors de « je " qui représentent sa conscience de soi le font paradoxalement se perdre dans la montagne et lui font craindre d'avoir perçu son " âme noire ». Dans le chapitre suivant, " tu » éprouve ainsi une sensation mortelle:

Une fois passée la première peur de la mort, une fois dissipée ton angoisse et calmée ton agitation, tu restes dans une espèce d'hébétude. Perdu dans la forêt vierge, tu erres sous des arbres morts, dénudés, prêts à tomber. Tu tournes longtemps autour de ce trident étrange qui semble te désigner le ciel sombre, n’osant pas t’éloigner de cet unique point de repère, dernier signal dont tu te souviens.

Mais tu ne veux pas rester échoué sur ce trident comme un poisson hors de l'eau; mieux vaut abandonner les dernières attaches qui te relient au monde plutôt que t'acharner à rassembler tes souvenirs. Tu peux te perdre davantage, mais tu veux conserver une dernière chance de survie. C'est parfaitement compréhensible ${ }^{54}$.

Déjà, dans le chapitre 10, "je " a vécu presque la même expérience, lorsqu'il s'est perdu dans la forêt:

Le brouillard gris est revenu dans mon dos, sans se soucier des fossés, des anfractuosités du terrain, des troncs d'arbres couchés. Je n'ai aucun moyen de fuir devant lui et il me rattrape, dans hâte. Je suis enfoui dans le brouillard. Le paysage a disparu devant moi, tout est indistinct. Seules restent dans ma tête les impressions visuelles que je viens de voir ${ }^{55}$.

[...]

Je me relève. J'attends, perdu. J'appelle, sans réponse. J'appelle une nouvelle fois, mais j'entends seulement ma propre voix triste et tremblante qui s'éteint. Toujours pas de réponse. Aussitôt la peur m'étreint. Elle monte depuis la plante de mes pieds et mon sang se glace. J'appelle encore, toujours sans réponse. Autour de moi, seule l'ombre noire des sapins, tous identiques. Je me mets à courir, je crie, je me précipite à gauche, à droite, je perds la raison. Je dois me calmer, revenir à mon point de départ, non, je dois d'abord essayer de m'orienter, mais, partout autour de moi, se dresse l'ombre des sapins

53. GAO, X. J., La Montagne de l'âme, op. cit., p. 94-97.

54. Ibid., p. 555.

55. Nous modifions la traduction de cette phrase d'après la version chinoise. 
noirs. Pas un seul point de repère. J'ai déjà tout vu, mais c'est comme si je n’avais rien vu. Les veines à mes tempes palpitent avec force. [...] Sur le ciel grisâtre se détache la silhouette singulière d'un arbre; il est penché, son tronc est divisé en deux parties de la même taille qui poussent droit, sans branches ni feuilles. Complètement dépouillé, il doit être mort; il ressemble à un harpon gigantesque et monstrueux désignant le ciel. Je me dirige vers lui. En fait, il est situé à la limite de la forêt. En dessous doit se trouver la gorge sombre, cachée par le brouillard; c'est donc une direction qui mène droit à la mort.

[...]

Tous les récits sur des personnes qui sont mortes égarées dans la montagne, dont j'ai entendu parler ces derniers jours, me reviennent à l'esprit et me terrorisent. Je me sens pris au piège. À cet instant, je ressemble à un poisson pris dans les filets de la peur, percé par un gigantesque harpon: il se débat sans pouvoir changer son destin, sauf par miracle. Mais dans ma vie, n'ai-je pas toujours attendu un miracle ${ }^{56}$ ?

Il semble que la fin du chapitre 65 et le début du chapitre 66 relancent l'expérience que « je » éprouve dans le chapitre 10, et font basculer la situation extérieure du chapitre 10 en celle intérieure du chapitre 66: en d'autres termes, la situation difficile de " je » dans le monde extérieur est transmuée en perception de " tu » dans le monde intérieur. Le moi ou bien la conscience du moi, qui est omniprésente dans la vision de « je ", commence à disparaître, d'abord par le détachement du corps, puis par celui de la volonté subjective.

À l'orée de la forêt, « tu » court vers le ravin sans savoir pourquoi et sans réfléchir, mais éprouve " un détachement comme tu n’en as jamais connu », puisqu'il ne porte plus son corps:

$\mathrm{Tu}$ as l'impression de planer, de te fendre en deux, de perdre forme humaine pour te fondre dans le paysage; parfaitement serein, voletant au milieu du profond ravin. Comme un fil flottant qui semble toi-même, se trouve dans un espace inexplicable où il est rempli par l'odeur de la mort: tu es tout froid ${ }^{57}$.

Lorsque « tu » ne cherche plus à être maître de lui-même — dans les registres du corps et de la volonté - sa perception ne s’attache

56. GAO, X. J., La Montagne de l'âme, op. cit., p. 94-97.

57. Ibid., p. 556. II y a une omission dans la version française par rapport à la version chinoise. 
plus à lui. Et cette perception impersonnelle révèle alors un moi de non-moi plus proche d'un « vrai » moi. Si le corps représente un lien naturel entre le moi et le monde extérieur, lorsque « tu » abandonne " les dernières attaches qui te relient au monde ", la sensation "sans corps » représente purement le monde intérieur du moi. De même, lorsque " tu » délaisse la volonté de choisir et d'atteindre un certain but, la vie n'est représentée que par une sensation intérieure qui ne dépend plus de lui mais lui révèle une autre vérité:

Tu voyages au Royaume des Morts, ta vie n'est plus entre tes mains, tu continues à respirer uniquement à cause d'un moment de frayeur, ta vie est suspendue entre l'avant et l'après de cette frayeur. Si tu glissais, si les cailloux que tu agrippes de tes doigts de pied roulaient, si ton pas ne pesait pas sur le dos de l'eau, tu sombrerais dans la rivière infernale, comme ces cadavres soupirants qui filent au gré du courant. Il n'y a pas davantage de sens, n’y prête pas attention, avance et c'est tout. Seuls demeurent le filet tranquille de la rivière, l'eau noire comme la mort, les feuilles, des branches qui frôlent la surface de l'eau, le courant qui s'écoule en longues draperies comme des peaux de loups morts, aux milieu de la rivière de l'Oubli.

Tu n'es guère différent du loup, tu as causé assez de fléaux, tu seras mis à mort par les autres loups, sans raison. Dans la rivière de l'Oubli, tout le monde est à égalité, la fin des hommes et des loups, c'est toujours la mort.

Cette découverte provoque en toi une certaine joie, une joie qui te donne envie de crier, mais ta gorge n'émet aucun son, le seul bruit que tu entendes, ce sont les coups sourds de l'eau sur les trous des racines des arbres.

D’où vient cette vacuité ${ }^{58}$ ? Les eaux sont sans limites, elles ne sont pas profondes, mais s'étendent à l'infini. La Mer des souffrances est sans limites aussi, et toi, tu flottes dans cette Mer des souffrances infinies $^{59}$.

58. Gao joue ici avec les mots. Voilà la version chinoise : 这发现令你多少有些快活, 你快活得想大喊一声, 喊叫又没有声音, 有声音的只是河水咕嘟咕嘟拍着树根 下的空洞。空洞又从何而来? 水域漫无边际, 并不很深, 却没有岸边。有个说 法, 苦海无边, 你就在这无边的苦海中荡漾。»

Le terme 空洞 peut signifier des choses concrètes comme les «trous», mais aussi un concept abstrait comme la vacuité, notamment dans le sens bouddhiste. Vu le contexte (il y a un autre terme «la mer des souffrances » (苦海) issu du bouddhisme), avec le deuxième 空洞 il s'agit plutôt de la vacuité. Nous modifions donc la traduction de Noël Dutrait.

59. GAO, X. J., La Montagne de l'âme, op. cit., p. 557-558. 
Le moi devient petit à petit transparent: le corps est délaissé pendant la transition de la vision extérieure de " je " à la vision intérieure de «tu »; la conscience du moi est négligée lorsque «tu » renonce à la volonté. Le moi qui implique la nature de la vacuité est à la fois invisible et visible: invisible parce qu'il ne se sent plus et ne se voit plus en tant que sujet concret ou subjectivité individuelle; visible parce qu'il ne se distingue plus des autres; il s'identifie au fil flottant, aux loups ou même aux eaux dans la Mer des souffrances; il se fait voir partout mais n'est pas dans sa propre vision ou dans une certaine vision. Et c'est au moment où le moi est ainsi visible et en même temps invisible que " tu " le ressent dans une mesure infinie.

\section{LE REGARD DU TROISIÈME EIL (第三只眼)}

\section{Entre moi unique et moi indistinct}

Certes, le moi chez Gao ne se réfère pas de manière univoque à une notion formée par la pensée du bouddhisme chán, dont le moi est pur et vide de façon innée. Il est influencé aussi par la pensée occidentale contemporaine, dont le moi est né du désir. Cependant, ces deux pensées opposées ne provoquent pas chez Gao le conflit de deux interprétations contradictoires concernant le moi. Elles font apparaître un œil qui vient du moi mais qui s’isole de ses propres émotions, un œil du moi qui le regarde de loin afin de l'éclairer dans sa dimension chaotique.

Gao montre que le moi est avant tout une existence qui va et vient entre les désirs variés de la chair, du pouvoir ou de l'ambition, et qui s'y enfonce: dans ce sens, à ses yeux, le moi est d'emblée un chaos. Pourtant, les désirs ne s'incarnent pas selon lui dans un caractère qui chercherait à se satisfaire, mais dans la narration d'une perception qui n'appartient pas vraiment à moi - il s'agit plutôt d'une perception impersonnelle. À partir de là, le moi chaotique se connecte avec le moi vide et transparent: le désir existe véritablement mais n'est pas forcément pour moi ou en moi, de même que le moi vide et transparent est là mais ne paraît pas en une substance/ corps. Pour ainsi dire, le désir n'est qu'une perception qui appelle la sympathie chez tout le monde aussi bien que chez moi. Tandis 
que le moi vide et transparent, comme la constitution des points d'acupuncture, est invisible et dépourvu de substance mais est bien informé de toute la perception du corps désirant.

Il s'ensuit que l'écriture chez Gao s'efforce, d'une part, de capter la sensation subtile et fugace, et d'autre part, tend à estomper la subjectivité au sein d'un sujet transparent, afin d'obtenir cette perception fluide qui se transforme sans cesse en saisissant la sensation « vivante » la plus fine et la plus variée:

Par l'écriture, on peut vivre des expériences qui nous sont interdites dans la vie normale. Il faut se détacher un peu de la vie matérielle pour capter cette perception subtile de l'esprit, qui est aussi une réalité, qu'on ne peut normalement pas connaître [...] C'est un moyen de pousser plus loin l'observation par les sensations, par le regard, l'ouie, le toucher, etc., pour connaître la vie d'une autre personne. Un univers que tu ne peux pas atteindre normalement ${ }^{60} \ldots$

Cependant, est-il possible d'avoir une écriture créative non-subjective? L'effacement de la subjectivité dans l'écriture de Gao ne risque-t-il pas de conduire à une langue banalisée et commune? Ou à l'« écriture automatique » comme chez les écrivains surréalistes? Denis Bourgeois interroge Gao sur ce point en 1994:

D. B. - L'écriture met à nu le soi, mais le soi s’avère une pseudo-entité, quelque chose qui se disperse à la mesure même de notre volonté de la définir. Si bien que l'écriture ne peut pas être subjective, au sens propre du terme, ni même objective; elle deviendrait simplement une traversée dans la langue par la langue.

G. X. - On peut approfondir la subjectivité. Cette subjectivité peut se présenter sous la forme d'un narrateur neutre, un «il », comme un œil neutre, mais elle reste de toute façon l'activité d'un « soi ».

D. B. - Tu veux dire, aussi loin que tu ailles dans la mise à nu dans l'écriture, il y a toujours une subjectivité, même un mince filet qui reste à la base et tient le tout?

G. X. - Oui, car ce regard neutre n'est pas le même pour tous. On peut dépouiller le mieux possible cette subjectivité, mais si elle disparaît complètement, on ne sent plus. On entre plutôt dans un état de vide absolu, comme dans la méditation bouddhiste. Il n'y a plus d'image concrète, tout est flou, vide, c'est l'état idéal pour les bouddhistes. C'est l'état qu'on appelle « oubli de soi ». Il n’y a plus d'émotion, il n'y

60. GAO, X. J., Au plus près du réel, op. cit., p. 124-125. 
a plus d'angoisse, même il n'y a plus de joie, aucune couleur de sentiment. C'est vivant, mais c'est imperceptible. Dans cet état-là, on est incapable de faire autre chose, donc a fortiori on ne peut pas écrire ou raconter une histoire. Moi, ça m’arrive quelquefois, je n’écris plus, je reste longtemps sans rien penser. C'est de la méditation zen [chán]. Parfois c'est nécessaire dans une société aussi intense que la nôtre. Mais je ne suis pas bouddhiste, ce n'est pas mon idéal de vivre dans cette vie de l'âme.

D. B. - Et c'est quoi, ton idéal?

G. X. - Je suis quand même un être vivant. Si on n'est pas dépressif, on aime quand même cette vie, bien qu'elle soit pleine de difficultés et qu'elle nous donne tant de mal. Pourquoi ne pas affronter aussi cette angoisse ${ }^{61}$ ?

La question que Bourgeois pose sur la subjectivité est sans doute pour lui incontournable, mais elle prend un sens différent chez Gao. Pour Bourgeois, la subjectivité s'établit sur la relation inséparable sujet/objet (la disparition de l'un signifie également l'annulation de l'autre), et se caractérise par sa singularité (une écriture non subjective n'est qu'une langue commune), alors que la subjectivité dont Gao parle ne peut être classée dans le cadre ni de l'un ni de l'autre: il s'agit d'une « subjectivité » qui non seulement est indifférente à l'objet (l'autre), mais encore ne se met pas en face de l'autre et reste indifférente à son statut de sujet. Lorsque Gao transforme la phrase fameuse de Sartre "L'enfer, c'est les autres " en "L'enfer, c'est le moi », il révèle déjà, d'une part, une " négligence » de l'attention pour l'autre qui découle de la pensée traditionnelle chinoise, et qui permet au moi de se trouver en dehors de la relation sujet/objet ou je/autrui; d'autre part, un moi qui n'est plus dans la disponibilité taoïste, mais anxieux.

Comme nous l'avons dit, le concept de l'autre n'est pas vraiment exploré à la façon philosophique occidentale dans la pensée chinoise. Lorsque Zhuangzi et les maîtres chán parlent de l’Un, il s'agit de l'égalité ou de l'indifférenciation (齐一); cela ne peut être comparé avec l'Un chez Aristote qui est synonyme d'Être, et s'implique spontanément dans ce rapport avec le multiple et les multiples $^{62}$ qui reflète effectivement la relation du moi avec autrui ou

61. Ibid., p. 129-130.

62. Aristote, Métaphysique, Livre iota (X), chap. 2, 1053b 16 et 1054a 13. 
les autres. C'est pourquoi, le dépassement dans le taoïsme, le bouddhisme chán et même le confucianisme ne s'adresse qu'au soi-même ou au rapport entre soi et ciel/nature - où autrui n'existe qu'à l'état embryonnaire. La vacuité de la nature du soi, en tant qu'essence de chaque individu, s'identifie à l'essence de l'univers: car seul le vide et le « il n'y a pas " qui ne cherchent pas à se construire contiennent en même temps tout. Autrement dit, la vacuité révèle un " axiome " de l'indifférenciation qui signifie "Un » de non-deux (不二) et qui tente d'effacer la différence entre soi et les autres, entre sujet et objet afin de dépasser le dualisme et de voir la tathata (真如). C'est dans cet esprit qu'au IV ${ }^{\mathrm{e}}$ siècle le moine Shengzhao (僧肇), premier disciple du grand traducteur Kumarajiva, déclarait: "Je partage la même origine du ciel et de la terre, je m'identifie à toutes les choses ${ }^{63}$ (天地与我同根, 万物与我一体).”

Dans la philosophie et la littérature occidentales, le concept d'autre n'est jamais absent: car c'est l'autre qui me reflète et $m e$ fait connaître moi-même. Deleuze y insiste en partant de Proust, il y a une différence " ultime et absolue ", qui révèle une essence à travers l'œuvre d'art. Cette différence,

[...] non pas une différence empirique entre deux choses ou deux objets, est toujours extrinsèque. Proust donne une première approximation de l'essence, quand il dit qu'elle est quelque chose dans un sujet, comme la présence d'une qualité dernière au cœur d'un sujet: différence interne, différence qualitative qu'il y a dans la façon dont nous apparaît le monde, différence qui, s'il n'y avait pas l'art, resterait le secret éternel de chacun.

À cet égard, Proust est leibnizien:

Les essences sont de véritables monades, chacune se définissant par le point de vue auquel elle exprime le monde, chaque point de vue renvoyant lui-même à une qualité ultime au fond de la monade. Comme dit Leibniz, elles n'ont ni portes ni fenêtres: le point de vue étant la différence elle-même, des points de vue sur un monde supposé le même sont aussi différents que les mondes les plus lointains ${ }^{64}$.

63. SHENGZHAO, 《肇论校释》 (Zhaolun jiaoshi), Beijing, Zhonghua Shuju (中华书局), 2010， p. 283-294.

64. Deleuze, G., Proust et les signes, op. cit., p. 54. 
Deleuze établit son argumentation sur l'inséparabilité de la subjectivité et de la perspective ${ }^{65}$ qui lui est propre: une idée fondamentale dans la littérature occidentale. Déjà, on trouvera un écho contemporain à Proust, sous la plume de Remy de Gourmont, qui pour dessiner le portrait de la littérature symboliste, cite l'expression vulgarisée de Schopenhauer: "Le monde est ma représentation. Je ne vois pas ce qui est; ce qui est, c'est ce que je vois ${ }^{66}$. " Dans l'Un du bouddhisme chán, la perspective ne se rattache pas forcément au sujet, et est encore moins considérée comme l'essence de l'individu.

Gao oscille entre les deux pensées. L'autre est à la fois présent et absent chez lui. Comme Jin Siyan le dit justement chez Gao, «l'autre n'est pas seulement à l'extérieur, mais aussi chez soi ${ }^{67}$ ". Dans un entretien avec Daniel Bergez, Gao parle de son troisième œil pour répondre à la question que Bergez lui pose sur la pluralité des points de vue d'un sujet:

Ce regard, je l'appelle souvent le " troisième œil »; il observe et éclaire le moi chaotique. C'est avec lui qu'on trouve la forme et l'expression artistique. L'artiste est aussi un observateur; tout en travaillant il regarde ce qu'il fait. Il voit d'abord avec un œil d'observateur créateur; il voit ensuite avec un œil de spectateur tout simple. Avec son double regard, le peintre sait si ce qu'il a senti peut être regardé par l'autre. La conscience du peintre est aussi dans la peinture. Mais ce n'est pas une réflexion; c'est un regard. Il observe celui qui regarde ${ }^{68}$.

Ce regard qui provient du moi mais considère le moi comme un autre s'incarne à la fois dans la perspective du moi (le créateur) et dans celle de l'autre (le spectateur), puisque moi et ma perspective ne sont pas inséparables. C'est à partir d'ici que Gao parvient à unir l'égalité, ou l'indifférenciation (齐一), et la singularité (独一) qui représentent respectivement la nature du soi vide dans la pensée de Zhuangzi et du bouddhisme chán, et celle du moi chaotique dans

65. Descombes, V., Proust, philosophie du roman, Paris, Minuit, 1987, p. 47-48.

66. Gourmont, R. de, Le livre des masques, Paris, Mercure de France, 1896, troisième édition, p. 11-12.

67. JIN, S. Y., L'Écriture subjective dans la littérature chinoise contemporaine: devenir je, Paris, Maisonneuve \& Larose/Espace du temps présent, 2005, p. 221

68. «Le troisième œil, entretien avec Gao Xingjian », dans Europe, Janvier-février 2007, $n^{\circ}$ 933-934, p. 247-256. 
la littérature occidentale contemporaine et aussi dans son propre concept du moi. Le soi vide qui tend à s'oublier et à se dépasser est plutôt comme une idée qui fascine et même dirige la création de Gao, mais le moi chaotique - en tant que sujet du penser, du parler et du voir - tient à sa singularité comme à son caractère essentiel, et possède un "corps " réel dans ses ouvrages. Si je peux être moi, c'est parce que ce moi est unique, qu'il est différent des autres; mais lorsque " je ", " tu ", " elle " ou « il » sont des figures des autres et en même temps ne sont que les autres moi, la différence entre moi/ autre est estompée. Ainsi le troisième œil qui implique la séparation de la subjectivité et de la perspective, et observe de loin le moi chaotique, se donne la possibilité de reposer sur l'« entre » des deux polarités, celles du moi unique et du soi indifférencié.

\section{Un regard froid du moi sur moi-même sans rapport à la subjectivité}

En réalité, le troisième œil, appelé aussi l'œil de la sagesse ou l'œil neutre, est encore une notion issue du bouddhisme ${ }^{69}$ : c'est la désignation d'une vue pénétrante qui affranchit de l'illusion, et permet de voir la vacuité. Chez Gao, le troisième œil représente une vue de ce type, mais ce qu'il tente de dépasser est bien différent de ce que préconise le bouddhisme:

L'œil neutre, c'est-à-dire le troisième œil ou l'œil de la sagesse, en excluant la sensation subjective, la préférence personnelle et le juge-

69. II existe cinq genres d'yeux et donc cinq niveaux de vue d'après l'un de plus courts mais plus importants sutras, le sūtra du diamant (金刚经): I'œil de chair, celui des êtres humains ; l'œil du ciel, celui des célestes ; l'œil de la sagesse, celui du sage ; l'œil du dharma, celui du Bodhisattva; i et finalement, l'œil du Bouddha. Selon le bouddhisme, l'œil de chair est aveuglé par les illusions; avec l'œil du ciel, on peut voir tout de l'intérieur autant que de l'extérieur, mais il est obscurci par les idées fausses (理障), et ne peut voir la « vérité ». L'œil du Bodhisattva et l'œil du Bouddha sont deux vues supérieures; notamment, la vision du Bouddha représente une capacité suprême de voir la "vérité » absolue de tous les dharmas. Voir Soûtra du Diamant, traductions du tibétain par Philippe Cornu, Fayard, 2001; Le Sūtra du Diamant, traduction du chinois par Jin Siyan, Youfeng, 2007, Exégèses sur le Sūtra du Diamant, Maitre Hsing Yun, Youfeng, 2007. Dans le chapitre 30 du Sütra de vie infinie (无量寿经), l'œil de la sagesse a la capacité de voir la vérité de la vacuité : II ne voit pas ce qu'« il y a », ni ce qu'« il n'y a pas ». Pourtant, il ne s'agit pas d'une vue aveugle, mais de celle qui dépasse ce que l'œil de chair ou l'œil du ciel ont vu, et il le voit comme une forme de l'indifférenciation. 
ment éthique et politique, n'est pas manipulé par certaines valeurs a priori, ne fait que l'observation lucide ${ }^{70}$.

Lorsque Gao nomme ce regard le troisième œil/l'œil de sagesse/ l'œil neutre, il lui donne d'emblée trois particularités: la position d’observateur extérieur du moi; la manière de le pénétrer par la sagesse et non pas par la connaissance accumulée; enfin, l'attitude du sujet regardant ne s'engage pas dans l'émotion. Ainsi, le troisième œil révèle une observation du moi sur moi-même, elle n'est pas entravée par les jugements extérieurs, ni par le sentiment personnel intérieur.

Gao soutient que la poétique moderne est caractérisée par une sorte de "lyrisme froid ", dans un long dialogue de 1987 avec son vieil ami le poète Ma Shoupeng. Il reparlera plusieurs fois dans les années suivantes de ce "lyrisme froid " qui, pour lui, est une manière correcte d'exprimer concrètement le regard neutre, puisque le lyrisme émotionnel ne traduit que l'amour narcissique du soi-même. Il le met encore en avant dans le discours prononcé à Stockholm pour la réception du prix Nobel en 2000:

La poésie n'émane pas seulement du lyrisme. Le narcissisme sans limites de l'écrivain est une sorte de maladie infantile; certes, lorsqu'on apprend à écrire, on ne peut l'éviter. De plus, l'expression des sentiments possède de multiples niveaux; mieux vaut une contemplation d'un regard froid qu'une hauteur d'esprit exagérée. C'est dans cette observation distanciée que se dissimule la poésie. Et si ce regard qui observe examine aussi l'écrivain lui-même, et que de la même manière il se place au-dessus des personnages du livre et de l'auteur, il devient le troisième œil de l'écrivain, un regard le plus neutre possible. Alors, les catastrophes et les immondices du monde des hommes pourront aussi être examinées, et tout en provoquant souffrance, dégoût et nausée, elles éveilleront pitié, amour de la vie et attachement ${ }^{71}$.

Le troisième œil est donc un regard vigilant fixé sur le moi pour que l'auteur n'abuse pas de son émotion dans l'écriture lorsqu'il réexamine sa vie. Dans le roman classique Le Rêve dans le pavillon

70. GAO, X. J. et FonG, G. C. F, «论戏剧 » (Du théâtre), Taibei, Lianjing (联经), 2010, p. 34.

71. GAO, X. J. « La Raison d'être de la littérature », dans Le Témoignage de la littérature, op. cit., p. 117. 
rouge, le roc/jade en tant que héros qui ouvre le premier chapitre s'implique dans une vision similaire à celle que Gao propose même si elle n'est qu'embryonnaire ou entrevue dans ce grand ouvrage. Le roc abandonné par Nüwa pour réparer le ciel s’afflige pourtant jour et nuit, une fois fondu par le feu et pénétré d'une nature spirituelle, de son destin. Un jour, lorsqu'un bonze bouddhiste et un moine taoïste passent au-dessous de lui, il leur demande de l'emmener dans le monde humain en le transformant en un joli jade. En tant qu'autre moi de Baoyu (le protagoniste du Rêve dans le pavillon rouge), le jade qui est dans la bouche de Baoyu à sa naissance vient avec lui dans le monde. Il est porté par Baoyu comme le bijou le plus précieux et présente ainsi une autre vision du «moi/ Baoyu » qui va de l'extérieur (le monde) vers l'intérieur (le moi), et complète la vision de Baoyu de l'intérieur (le moi) à l'extérieur (le monde).

Le troisième œil chez Gao s'incarne aussi dans un regard provenant du moi mais qui se trouve en dehors du moi, et représente un regard non subjectif. Certes, ce regard de l'extérieur vers l'intérieur ne s'identifie pas à la voix off du narrateur « je » invisible (par exemple chez Stendhal dans les Chroniques italiennes et le premier chapitre de La Chartreuse de Parme), mais plutôt à une conscience lucide et impersonnelle. En d'autres termes, à la différence du regard du jade dans Le Rêve dans le pavillon rouge, ce regard ne provient pas du corps du moi, mais d'un moi sans chair: c'est un regard qui ne dépend en rien d'un être substantiel.

Dans Le Livre d'un homme seul, le narrateur « je " qui a disparu révèle à la fois l'impossibilité de vivre en tant qu'homme libre à l'époque de la révolution culturelle, et l'intention de l'auteur de se retirer de toute émotion du "moi ». Cependant, le regard/la conscience de "je " se manifeste dans le roman lorsque " tu " et « il ", les deux moi, le moi dans le présent et le moi dans le passé, prennent leurs distances avec eux-mêmes et se fixent l'un sur l'autre:

Tu dois trouver un ton pondéré, étouffer la colère accumulée en toi, avancer tranquillement, pour raconter calmement ces impressions mêlées, ces souvenirs qui affluent à flot continu, ces pensées dans lesquelles tu ne vois pas clair, et tu découvres à quel point c’est difficile. 
$[\ldots]$

Son expérience passée s'accumule dans les replis de ta mémoire. Comment faire pour les dérouler couche après couche, les dissocier pour les explorer un à un, et considérer d'un regard froid les événements qu'il a vécus: toi c'est toi, lui c'est lui. Et toi, tu as beaucoup de peine à revenir à son état d'esprit d'alors, il est devenu si étranger pour toi aujourd'hui, tu ne dois pas le surcharger de ton autosatisfaction et de ton contentement actuels, tu dois garder une distance, refouler tes émotions, pour mieux l'examiner. Tu ne dois pas confondre ta fureur avec sa vanité et sa stupidité, tu ne dois pas non plus masquer sa peur et sa lâcheté, tout cela est si difficile, cela te plonge dans un cafard noir. Tu ne dois pas non plus glisser graduellement dans son amour de lui-même et son masochisme, tu n'as qu'à observer et écouter attentivement et ne pas être attiré par ce qu'il ressent.

Tu dois laisser sortir de ta mémoire ce « il », cet enfant, cet adolescent, cet homme qui n'est pas devenu adulte, ce rescapé qui rêvait en plein jour, ce disciple de l'extravagance [...].

Tu ne joues pas le rôle du juge et tu ne le considères pas comme un sinistré, la fureur et la douleur qui nuisent à l'art doivent céder la place à l'observation; ce qui est intéressant, ce ne sont pas ton jugement et sa juste indignation, ce ne sont pas non plus ta tristesse et sa douleur, ce qui compte, c'est le processus d'observation en lui-même ${ }^{72}$.

L'un des meilleurs amis de Gao, le professeur et critique de littérature Liu Zaifu, estime que « tu » représente le regard du troisième œil, puisque «tu " se détache l'expérience de l'auteur dure la révolution culturelle ${ }^{73}$. Mais, faut-il ajouter selon Liu Zaifu, «il » ne représente pas forcément l'expérience de l'auteur dans le passé ${ }^{74}$. Si l'histoire de "il " est une création esthétique à partir de la fiction et de l'expérience de Gao, alors qu'il n'y aurait pas de raison pour que l'histoire de " tu » ne soit pas issue de la même autobiographie fictionnelle. En effet, si le troisième œil n’est jamais représenté par un personnage, la transition entre " tu » et « il » est fondée très exactement sur ce regard neutre. Rappelons une transition similaire entre l'autobiographie déguisée de l'auteur et celle fictive du personnage qui dit « je » dans À la Recherche du temps perdu de Proust (elle est

72. GAO, X. J. Le Livre d'un homme seul, op, cit., p. 237-238.

73. LIU, Z. F., (刘再复) 《高行健引论》(Gao Xingjian Yinlun), Hong Kong, Dashan wenhua chubanshe, 2011, p. 190-193.

74. Ibid., p. 193. 
fondée sur l'enjeu du temps et sur la mémoire involontaire ${ }^{75}$ ). Dans Le Livre d'un homme seul, au moment où « tu » ou « il " se distinguent d'eux-mêmes en veillant à ne pas être manipulés par leurs propres émotions et sentiments, ils prennent tous les deux un regard impersonnel. C'est pourquoi le troisième œil est indépendant de la vision du moi/narrateur et de toute substance, et n'est obscurci ni par le temps ni par l'espace. Si le chapitre 22 représente un regard perceptible du troisième œil lorsque « tu " prend de la distance avec lui-même et fixe son regard sur « il », le chapitre 31 montre un même regard imperceptible de " il » jeté sur « tu »:

Dans le lointain incertain, une lumière semble scintiller, tu te diriges - mieux vaut dire que tu rampes - vers elle, la lumière brille à travers une fente, c'est une maison, une porte, tu rampes jusqu'à cette porte, tu tends la main pour la toucher, tu parviens enfin à l'ouvrir, tu entends le bruissement du vent, mais il n'y pas de vent, dans un grand hall, un cercle de lumière t'éblouit, tu rampes pour atteindre ce cercle, puis tu te mets debout sur un solide plancher de bois, et là tu découvres que tu es nu comme un ver, mais devant tu ne vois rien...

$\mathrm{Tu}$ as besoin de prendre une contenance, puis de ne plus bouger, de te transformer en statue;

tu as besoin, tel un fil de la vierge, de flotter dans les airs et de disparaître peu à peu comme un lambeau de nuage;

[...]

tu as besoin de couler lentement au fond de l'eau, dans l'obscurité, comme un bateau qui sombre, et tu veux voir les bulles remonter à la surface, dans le plus profond silence;

tu as besoin de devenir un poisson à grosse tête qui évoluerait parmi les algues, agitant la tête, balançant sa queue;

tu espères devenir un œil triste, profond et désolé, et contempler de cet œil comment tourne et retourne le monde, et cet œil est au centre de ta paume;

[...]

$\mathrm{Tu}$ voudrais te mettre à pleurer en hurlant devant tout le monde, mais sans bruit, les gens ne sauraient pas pourquoi tu pleures, ils ignoreraient si tu pleures vraiment ou si tu fais semblant, tu voudrais aussi pleurer un bon coup sur ce monde apprêté, toujours sans bruit, en jouant l'homme qui pleure, laissant perplexe les respectés specta-

75. Voir Ricoeur, P., La Configuration du temps dans le récit de fiction, et DELEUZE, G., Proust et les signes. 
teurs, puis tu voudrais te déchirer la poitrine et en retirer un cœur de plastique rouge, puis une poignée de papier de riz ou de papier hygiénique et les lancer vers ceux qui acceptent de t'acclamer, avancer d'un pas souple, et ensuite, ensuite, glisser à terre et ne plus pouvoir te relever, mourir d'un infarctus sur scène, en réalité tu n'as pas besoin de secours, tu ne fais que jouer la comédie, tu voudrais juste, comme ça, montrer la peine et la joie, la tristesse et le désir, avec un petit sourire plein de ruse, sourire ou grimace? [...]

On peut aussi changer d'angle de vue, tu te trouves parmi les spectateurs, tu le regardes monter sur la scène, une scène vide, il se tient debout, nu, et sous la lumière crue des projecteurs, il doit pendant un instant s'habituer à cette lumière avant de pouvoir, à travers le faisceau lumineux éclairant la scène, te distinguer toi, assis sur un fauteuil de velours rouge au dernier rang au fond du théâtre vide aussi ${ }^{76}$.

La proposition du dernier paragraphe de changer la place du spectateur de « il » en " tu » dévoile que dans les paragraphes précédents, « tu » est sous le regard de « il » invisible, et « il », en tant que vue pénétrante, voit les envies et les fantasmes les plus profonds de " tu », voit ce « tu » qui est torturé par ses propres désirs.

De plus, le chapitre 5 présente aussi un regard invisible du troisième œil qui ne dépend ni de « il » ni de " tu » lorsqu'ils se distinguent eux-mêmes. Cette invisibilité du regard est perçue dans un autre jeu de changement de temps et d'espace: toujours dans le chapitre 5, quand Gao met «il » dans le temps présent et qu'il vient de se réveiller du cauchemar concernant sa vie du passé:

Il est chez lui, à Pékin, il ne sait comment il est revenu. Il n'arrive plus à trouver la clef de son appartement pour ouvrir la porte, il est très inquiet, il a peur que les habitants de l'immeuble ne le reconnaissent. [...] Il se réveille, c'est un cauchemar.

Cela faisait longtemps qu'il n'avait plus fait ce genre de cauchemar et même s'il lui arrivait d'en faire, ceux-ci n'avaient à présent plus aucun rapport avec la Chine. [...]

À présent, il est un oiseau libre. C'est une liberté intérieure, il n'a plus le moindre souci, il est libre comme l'air, comme le vent ${ }^{77}$. [...]

Puis " il " ferme les yeux, "laisse divaguer son esprit ", va et vient entre plusieurs époques de son passé. Entre-temps, il paraît

76. GAO, X. J. Le Livre d'un homme seul, op, cit., p. 317-320.

77. Ibid., p. 51-53. 
qu'il y a toujours un œil qui regarde cet « il »: celui-ci n'est pas un homme spontané mais un «il » introspectif qui est remis dans les différents temps par ce regard. Le chapitre 46 donne un exemple réciproque du retour de « tu » dans le temps du passé:

$\mathrm{Si}$ tu avais appris à regarder le monde avec le visage hilare du Bouddha Amithaba, tu serais toujours heureux, la paix régnerait dans ton cœur et tu atteindrais le nirvana.

Tu mangeais et tu buvais avec les cadres du pays, tu les écoutais dire leurs bêtises, fanfaronner et parler des femmes. [...]

Voilà la vie, on n'était heureux qu'après avoir bu sa part d'alcool! Il te faudrait aussi leur parler du moyen de récupérer un sapin pour fabriquer des coffres et trouver du bois bon marché, au prix public d'achat, parce que, un jour ou l'autre, tu devrais te construire une maison puisque tu t'étais installé ici ${ }^{78}[\ldots]$

Soit représenté par " tu » ou « il » lorsqu'ils se détachent d'euxmêmes, soit impliqué dans une conscience ou un esprit invisible, le regard du troisième œil se place derrière et au-dessus des narrateurs qui se plongent dans le mémoire du passé ou du présent, et les regarde de haut et de loin mais en même temps en pénétrant jusqu'au fond de leur monde intérieur.

\section{L'extension entre le monde extérieur et le monde intérieur: le paysage}

Si le troisième œil déchiffre un regard à la fois du moi et du "non moi " sur le monde intérieur du moi, le monde extérieur, pour sa part, est vu chez Gao de façon allusive et indirecte. Il y a un exemple remarquable de vision à travers l'objectif de l'appareil photographique dans Le Livre d'un homme seul:

Mais tu dis que ce que tu veux, c'est un réel limpide, comme un tas d'ordures saisi par un objectif, l'ordure reste l'ordure, mais à travers l'objectif elle porte ta tristesse. Le réel, c'est cette tristesse. Tu t'apitoies sur ton sort, il te faut chercher un état d'esprit qui te permette d'accepter la souffrance, afin de continuer à vivre, tu inventes un Monde (境界) qui n’appartiendrait qu'à toi, hors de cette réalité semblable à une porcherie ${ }^{79}$.

78. Ibid., p. 439-440.

79. Ibid., p. 427. 
Ce regard à l'aide de l'objectif est « limpide » en termes de constatation du réel mais nuancé par la sensation de l'observant à partir de laquelle « tu » a l'intention d'inventer un Monde. Lorsque Gao utilise le terme " $\operatorname{Monde}^{80}$ (境界) " au lieu de 《monde (世界) " au sens d'univers, il ne s'agit certainement pas d'un monde idéal du côté de la raison, mais d'un monde esthétique du côté de la réalité:

Dès qu'on le regarde à travers un objectif, le monde change, les choses les plus laides peuvent devenir magnifiques. À l'époque, tu avais un vieil appareil photo et pendant ces années passées à la campagne, tu l'emportais chaque fois que tu allais en montagne, c'était ton autre œil. Tu as photographié les paysages, la montagne couverte de bambous courbés sous le vent, cette vague verte émeraude en forme de plume fixée sur le papier par le claquement de l'obturateur. La nuit, tu développais les photos chez toi, et, même si tu perdais les couleurs, les ombres et les lumières des noirs et blancs contrastés étaient fascinantes, on eût dit un monde de rêve ${ }^{81}$.

En effet, « tu » exprime son remords que « il », son moi du passé, n’ait pas protégé son élève Sun Huirong aussi bien que les deux jeunes institutrices pendant la révolution culturelle: le souvenir est comme l'appareil photo à travers lequel la réalité « laide » est devenue paysage.

Cependant, le fait que l'une « devient » l'autre ne résulte pas du reflet miroir mais de l'extension de l'intérieur vers l'extérieur et de l'extérieur vers l'intérieur. La montagne couverte de bambous verts vus en noir et blanc traduit l'affection de " tu » qui s'allonge et s'intègre dans ce paysage extérieur. En effet, la tristesse de " tu » s'étend à travers l'objectif jusqu'à ce qu'il voie dans le monde extérieur, et révèle exactement que l'objectif ne fonctionne pas comme un miroir

80. 境界/意境 (Monde) est une notion qui se forme sous la dynastie Tang et qui s'est enracinée dans la littérature chinoise depuis plus de mille ans comme catégorie esthétique essentielle, réinterprétée par Wang Guowei en combinant les pensées esthétiques chinoise et occidentale (notamment sous l'angle de la philosophie de Schopenhauer) au début du $x x^{e}$ siècle. Le point de vue de Wang est repris par beaucoup de chercheurs chinois contemporains comme une théorie qui analyse la notion 境界/ 意境 (Monde), par exemple chez Ye Lang (叶朗), 《中国美学史大纲》 (Aperçu de l'histoire de l'esthétique chinoise), Shanghai, Shanghai Renmin Chubanshe (上海人民 出版社), 1985, p. 609-611. Pour ne pas confondre 《境界》 avec monde (世界), nous le traduisons par Monde.

81. GAO, X. J. Le Livre d'un homme seul, op, cit., p. 453. 
afin de montrer à « tu » une image réflexive; et que le monde extérieur n'est pas le reflet du monde intérieur, et réciproquement; mais qu'ils sont l'extension de l'un et l'autre. Le Monde esthétique qui est construit par le paysage est à la fois l'intermédiaire et le connecteur du monde extérieur et intérieur, il se situe dans la zone extensive de l'un vers l'autre:

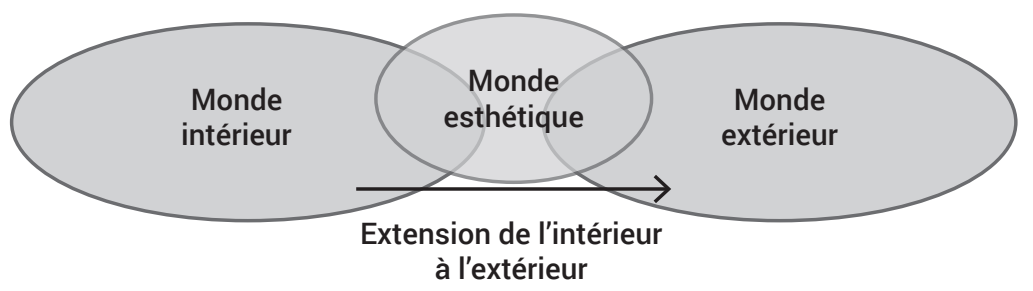

Voyons un exemple dans La Montagne de l'âme. Les chapitres 18 et 19 offrent deux récits qu'il convient de lire ensemble. Car la transition de la narration de " je " à " tu " est réalisée par un processus extensif du regard. La fin du chapitre 18 montre une situation difficile de « je » qui s'enfonce dans la vase pour monter dans un bateau:

Je me dis que je n'ai qu'à pousser le bateau hors des touffes de roseaux pour gagner cette vaste étendue. En me laissant flotter seul au milieu du lac, dans ces hauts plateaux isolés et paisibles, je n'aurai à parler à personne. Et j'aimerais me fondre dans le paysage pour ne faire qu'un avec la lumière, le ciel et les couleurs de la montagne ${ }^{82}$.

Nous l'avons vu précédemment, la situation sans issue fait que « je » éprouve paradoxalement la révélation instantanée (顿悟) en transformant l'impasse extérieure en calme intérieur; le paysage est la cause de l'enfoncement dans la vase mais aussi du pouvoir d'en sortir. Le chapitre 19 commence par une vue du paysage d'automne qui marque une extension du monde extérieur au monde intérieur:

Un soir glacial, au cœur de l'automne ${ }^{83}$. Une épaisse et profonde obscurité noie l'étendue chaotique originelle, le ciel et la terre, les arbres et les rochers se fondent, la route est invisible, tu ne peux que rester sur place sans pouvoir dégager tes pieds, le buste penché en avant, les bras étendus pour tâtonner dans cette nuit noire, tu entends bouger

82. GAO, X. J. La Montagne de l'âme, op, cit., p. 161-162.

83. Dans la version chinoise, il y a ici une virgule; on garde le point final selon la tradition française. 
(流动), mais ce n'est pas le vent, c'est l'obscurité dans laquelle il n’y a plus ni haut ni bas, ni gauche ni droite, ni lointain ni proche, ni aucun ordre déterminé, tu te fonds totalement dans ce chaos, tu sais seulement que ton corps possède un contour, mais même ce contour s'estompe peu à peu dans tes pensées, une lueur monte à l'intérieur de toi, comme le feu solitaire d'une bougie dans l'obscurité, sa flamme dégage de la lumière mais pas de chaleur, une lumière glaciale qui emplit ton corps, déborde ses contours, ces contours que tu conserves en pensée, tes deux bras se resserrent pour préserver ce feu, cette conscience glaciale et transparente, tu as besoin de cette sensation, tu t’efforces de la protéger, devant toi apparaît la surface tranquille du lac et, sur l'autre rive, se dressent des bosquets d'arbres, des arbres qui ont perdu leurs feuilles, et d'autres, pas encore complètement dépouillés, des peupliers sveltes où restent accrochées quelques feuilles jaunes, des jujubiers d'un noir métallique où seules une ou deux feuilles jaune pâle tremblent au vent, des arbres à suif pourpres clairsemés, semblables à des volutes de brouillard, à la surface du lac, aucune vague, seulement des reflets, nets et brillants, aux couleurs chatoyantes, du rouge sombre au pourpre, à l'orangé, au jaune tendre, au vert foncé, au brun gris, au blanc de lune, sur plusieurs niveaux, tu réfléchis intensément, puis soudain les couleurs disparaissent pour se fondre en d'innombrables nuances de gris, de noir et de blanc foncé ou clair, comme une vieille photo défraîchie, seules les ombres restent nettes, au lieu de dire que tu es sur terre, mieux vaut dire que tu es dans un autre espace, tu observes la propre image de ton cœur en retenant ton souffle, tout est si calme, le calme te rassure, tu as l'impression qu'il s'agit d'un rêve, qu'il ne faut pas t'inquiéter, mais tu ne peux t'en empêcher, justement parce que le calme est trop parfait, un calme exceptionnel ${ }^{84}$.

Une seule et longue phrase, qui montre la transition du paysage extérieur à la conscience de «tu » puis au paysage mental, provoque un mouvement d'extension continue. Le paysage du lac s'étend jusqu'à l'intérieur de " tu » et s'y répand: " tu te fonds totalement dans ce chaos ". Puis " une lueur monte à l'intérieur de toi " et lui fait se ré-éprouver lui-même, et pénétrer, à son tour, dans le paysage. À travers cette lumière/conscience glaciale et transparente, " tu » voit son image mentale dans la transition des couleurs du paysage lacustre. Ainsi, cette transition des couleurs révèle le mouvement du changement de ses états d'esprit successifs: du noir de

84. GAO, X. J., La Montagne de l'âme, op. cit, p. 163-164. 
l'obscurité aux multiples couleurs, puis aux « innombrables nuances de gris, de noir et de blanc foncé ou clair ", les différents niveaux des sensations/couleurs se transforment en montrant la zone subtile de la transition du chaos au calme exceptionnel et parfait. Un Monde esthétique (境界) s'établit dans cette extension du paysage vers " tu " et de " tu " vers le paysage, où le sujet " tu " s'estompe mais est exprimé par la description du paysage.

Cette extension réciproque a son origine dans la littérature chinoise traditionnelle où l'émotion de l'auteur s'exprime rarement de manière directe. Le monde intérieur de l'auteur se fait percevoir à travers un paysage extérieur qui est à la fois perceptif et affectif, et qui construit un Monde poétique (意境) non subjectif. On le trouvera chez beaucoup de poètes classiques, notamment chez Wangwei, le poète et peintre de la dynastie Tang. L'un de ses poèmes, Le Talus aux magnolias (辛夷坞) semble ne parler que du paysage:

À la cime des arbres, les fleurs de lotus.

木末芙蓉花

Dans la montagne, s'ouvrent de rouges corolles.

La hutte près du ravin, silencieuse et déserte.

Pêle-mêle des fleurs éclosent et se flétrissent ${ }^{85}$.

$$
\begin{aligned}
& \text { 山中发红萼 } \\
& \text { 润户寂无人 } \\
& \text { 纷纷开且落 }
\end{aligned}
$$

Le paysage, comme Wang Fuzhi le montre lorsqu'il commente les poèmes classiques de toutes les dynasties, est inséparable de l'émotion; c'est un caractère essentiel de l'esthétique chinoise: «le paysage donne la naissance de l'émotion, l'émotion conçoit le paysage $^{86}$ (景中生情, 情中含景) ». Autrement dit, le paysage n’est pas seulement objet regardé mais aussi sujet d'empathie. Les fleurs de lotus qui fleurissent et se flétrissent toutes seules dans la montagne que Wang Wei décrit, expriment et appliquent aussi le sentiment

85. Nous prenons la traduction de Wei-penn Chang et Lucien Drivod, en changeant le premier vers «À la cime des arbres on dirait des fleurs de lotus » en « À la cime des arbres, les fleurs de lotus». Voir Paysages: Miroirs du cœur (recueil des poèmes de Wangwei, traduit du chinois en français par Wei-penn Chang et Lucien Drivod), Gallimard, Paris, 1990, p. 215. Voir aussi «王维集校注 » Wangweiji jiaozhu, Beijing, Zhonghua shuju, 1997, p. 425. 86. WANG, F. Z., (王夫之), 《唐诗评选 » Tangshi pingxuan, cité par Ye Lang, dans «中国 美学史大纲 » Zhongguo meixueshi dagang, Shanghai, Shanghai renmin zhubanshe, 1985, p. 457. 
raffiné de la solitude de l'auteur. Au moment où l'émotion et le paysage s'intègrent, le Monde est là.

Il convient de distinguer l'effet miroir et l'effet de l'extension réciproque: le paysage et l'émotion ne se reflètent pas l'un l'autre, mais se conçoivent et s'étendent l'un dans l'autre: le sujet du sentir s'étend du moi aux fleurs de lotus/couleurs du paysage du lac; de même, la description du paysage s'inscrit dans mon monde intérieur. En d'autres termes, la subjectivité qui se fond dans le paysage rend manifeste une vision impersonnelle où le monde intérieur se laisse dessiner par le paysage extérieur: en ce sens, le sujet regardant est à la fois en dedans et en dehors du paysage et aussi de l'auteur.

Chez Gao, cette extension se prolonge encore entre les moi. En effet, le regard est une sorte d'extension qui fait se connecter " je", « tu » et " elle ». Dans le calme " parfait », « tu » voit le désir de " je " qui n'est pas exprimé dans le chapitre précédant: monter en bateau est en effet une allusion sexuelle, en suivant l'image du bateau qui s'étend jusqu'à ce chapitre:

Tu lui demandes si elle a vu cette ombre.

Elle dit qu'elle l'a vue.

Tu lui demandes si elle a vu le petit bateau.

Elle dit que c'est justement ce bateau qui donnait ce calme à la surface du lac.

Soudain tu entends sa respiration, tu étends la main pour la toucher [...]

Tu dis que tu veux que ce bateau coule.

Elle dit que le bateau est déjà plein d'eau.

Tu l'as écartée et tu es entré dans son corps humide ${ }^{87}$.

La sensation de l'amour physique est évoquée aussi par une extension de la vision:

Ensuite, ce sont les joues brûlantes, les langues de feu sautillantes, aussitôt englouties par les ténèbres, les corps qui se tordent, elle te dit doucement, elle crie qu'elle a mal! Elle se débat, te traite de bête sauvage! Elle est traquée, chassée, déchirée, avalée. Ah... cette obscurité dense, tangible, ce chaos germé, ni ciel ni terre, ni espace ni temps, ni être ni non-être, ni non-être ni être, ni être de non-être, ni être de

87. GAo, X. J. La Montagne de l'âme, op, cit., p. 164. 
l'être, le feu brûlant du charbon de bois, les yeux humides, la caverne ouverte, les volutes de fumée, les lèvres brûlantes, les cris gutturaux, l'homme et la bête, l'appel à l'obscurité originelle, l'angoisse du tigre féroce dans la forêt, l'avidité, les flammes sont montées, elle pleure en poussant des cris aigus, la bête sauvage mord, rugit, elle est ensorcelée, elle saute tout droit, tourne autour du feu, la lumière est de plus en plus claire, les flammes changeantes, informes, dans la grotte d'où s'élèvent les volutes de fumée, une lutte à mort s'engage, elle se précipite sur le sol, elle pousse des cris stridents, saute encore, rugit, étrangle et dévore... le voleur de feu s'est enfui, au loin la torche entre dans le noir, elle diminue, la flamme n'est plus qu'un petit point vacillant dans la bise. Elle s'éteint ${ }^{88}$.

Il y a un effet domino, dont le paysage est la clé, de l'extension visuelle entre "je ", " tu » et " elle ». Le bateau est la cause qui mène "je " à tomber dans une situation sans issue qui fait que "je » éprouve pourtant un sentiment de détachement. À partir de là, « tu " voit, dans le changement des couleurs du paysage lacustre, son image mentale qui s'étend jusque dans la vision de " elle ». C’est encore par l'image du bateau et des ténèbres sauvages que la vision s'étend vers le toucher, ou l'inverse.

L'extension entre les moi chez Gao nous offre ainsi un référent, voire une piste pour discerner comment les intellectuels chinois du $\mathrm{xx}^{\mathrm{e}}$ siècle déploient les plis de la notion du moi et les reconfigurent, et comment le dualisme moi/autrui occidental est traduit en relation réciproque de moi/paysage qui lie étroitement l'esthétique et la poétique dans leur tradition.

88. Ibid., p. 165. 


\section{5 \\ Langage et image}

La forme sous laquelle le langage s'exprime, définit par elle-même la subjectivité.

Jacques Lacan, Fonction et champ de la parole et du langage en psychanalyse

Ce qui parlait en elle, c'était l'approche, approche de parole, parole de l'approche, et toujours s'approchant, dans la parole, de la parole. Maurice Blanchot, L'attente l'oubli

J'aurais aimé m'apercevoir qu'au moment de parler, une voix sans nom me précédait depuis longtemps.

Michel Foucault, L'Ordre du discours

\section{LA SUBJECTIVITÉ ÉMERGE-T-ELLE DANS LE LANGAGE?}

\section{Pourquoi le courant de langage au lieu du courant de conscience?}

Comme pour beaucoup d'écrivains, ce qui est essentiel pour Gao est de trouver sa propre langue. Il écrit à la fois en chinois et en français, en respectant la structure et la grammaire propres de ces deux langues d'une façon très personnelle: lorsqu'il écrit en l'une de ces langues, il s'efforce d'oublier l'autre. Ainsi il met toute son attention à éviter dans son écriture un chinois occidentalisé. Selon lui, « utiliser des “traductions littérales” de langues occidentales pour enrichir 
la force d'expression du chinois moderne ${ }^{1}$ ", un phénomène de plus en plus à la mode, ne favorise en rien l'évolution de cette langue. Gao doute de cette intégration pour la raison que la différence entre la langue chinoise et les langues occidentales est radicale:

À l'origine, le chinois ne possède pas de normes grammaticales aussi strictes que celles des langues occidentales. La première grammaire du chinois, le Mashi Wentong (马氏文通), a été élaborée à la fin du siècle dernier. Elle emprunte les concepts grammaticaux des langues occidentales pour expliquer le chinois, alors que cette langue existe depuis plus de quatre mille ans ${ }^{2}$.

Cela ne signifie pas que Gao ait une attitude conservatrice à l'égard de la modernisation du chinois - au contraire, il s'est rendu compte très tôt de la nécessité de l'enrichir:

Dans les romans chinois traditionnels en langue parlée, le mouvement psychologique des personnages n'est mis en valeur que par leurs actions. Mais si l'on veut pénétrer dans l'activité psychologique de l'homme jusque dans son inconscient et recourir au langage pour en rendre compte, j'estime que le chinois tel qu'il existe n'est pas suffisant ${ }^{3}$.

Aussi Gao s'est-t-il orienté vers la recherche d'une expression psychologique qui soit en cohésion avec la structure linguistique du chinois:

Dans la littérature contemporaine occidentale, le courant de conscience cherche à saisir le flot psychologique à travers l'instant. James Joyce et Virginia Woolf ont chacun leur langage, mais je ne peux malheureusement en lire que des traductions et il m'est difficile de goûter vraiment la saveur de leur langue. Leur anglais est certainement très subtil. Marcel Proust et Samuel Beckett utilisent le même français rigoureux, mais ils expriment la psychologie à l'aide de procédés différents. Le goût, la saveur de cette langue, je peux les ressentir à partir du texte original. Mais comment trouver un chinois

1. GAO, X. J. «Le chinois moderne et l'écriture littéraire » (c'est un article que Gao a publié dans les actes d'un colloque à l'université de Provence), traduit du chinois par Noël Dutrait, dans Littérature chinoise, état des lieux et mode d'emploi, Aix-en-Provence, Université de Provence, 1998, p. 77.

2. Ibid., p. 75-76.

3. Ibid., p. 82. 
moderne qui traquerait le processus psychologique sans perdre la saveur de la langue ${ }^{4}$ ?

Dans les années 1920-1930, les écrivains de l'école de la Nouvelle Perception (新感觉派) avaient déjà expérimenté dans leurs récits les techniques symboliste, expressionniste et aussi psychologique suite à leur importation d'Occident et à la publication de traductions (la plupart du temps via le Japon). Mais par rapport à ses devanciers, Gao ne se focalise pas seulement sur la technique du style, mais aussi sur le rapport du moi et du langage: comment le moi se construit-t-il et s'exprime-t-il par et dans le langage à partir du fonctionnement de la langue chinoise? D’un ouvrage à l'autre, les expérimentations stylistiques de Gao intéressent toujours le rapport " moi-sujet parlant-parole parlée ». " Le courant de langage (语言流) » expression que Gao utilise pour désigner son style, est inspiré évidemment du courant de conscience mais s'en distingue: pour Gao, il est plus approprié de parler de « courant de langage " car le courant de conscience " ne peut être matérialisé, la seule chose qu'il puisse réaliser est un courant de langage ${ }^{5}$ ". Cet argument un peu hâtif ne rend pas vraiment compte des différences entre les deux " courants ", et ne permet pas suffisamment d'appréhender le fonctionnement du courant de langage. Il faut revenir aux ouvrages.

Une Canne à pêche pour mon grand-père (给我老爷买鱼竿), nouvelle écrite en 1986, représente l'embryon du courant de langage. L'histoire est simple, la première phrase peut même la résumer : " À la devanture d'une boutique d'articles de pêche récemment ouverte, la vue des nombreux modèles de canne à pêche exposés m'a soudain fait penser à mon grand-père ${ }^{6}$. » Le récit se déroule selon un monologue intérieur où le narrateur « je » remonte dans son souvenir aux hobbys de son grand-père décédé - la pêche et la chasse; beaucoup de paragraphes commencent par « je me souviens »:

4. Ibid., p. 82-83.

5. GAO, X. J. et YANG, L., Visite à Gao Xingjian et Yang Lian. Conversation, préface et traduction de Chantal Chen-Andro, Paris, éditions Caractères, 2004, p. 62.

6. GAO, X. J., Une Canne à pêche pour mon grand-père (recueil des nouvelles de Gao), traduit du chinois et préfacé par Noël Dutrait, L'Aube, 1997, p. 55. 
Je me souviens, quand j'étais petit — je me souviens parfaitement de tout ce qui concerne mon enfance - , si mon grand-père entendait dire que quelqu'un allait à la ville, il voulait absolument qu'on lui rapporte des hameçons, comme si les poissons ne mordaient qu'aux hameçons achetés en ville, je me souviens, il me répétait souvent qu'en ville on vendait des cannes à pêche équipées d'un moulinet [...] Je me souviens que c'est moi, en trébuchant, qui ai cassé sa canne préférée, nous allions à la pêche et je m’étais proposé de la porter, je courais devant lui, canne à l'épaule, quand je suis tombé dans un moment d'inattention, la canne fut projetée contre la fenêtre d'une maison, mon grand-père a failli en pleurer de chagrin, il caressait sa canne à pêche cassée de la même manière que ma grand-mère caressait sa vielle natte de bambou déchirée

[...]

Bien sûr je me souviens aussi, il y avait une rivière chez nous, j'avais l'impression qu'elle était loin, dans un lieu désert très éloigné de la ville, je me souviens, de toute mon enfance j'avais dû y aller une ou deux fois seulement et, quand mon grand-père était venu me voir, il m'avait dit que depuis, on avait construit un barrage en amont et que la rivière était asséchée, pourtant j'avais quand même envie d'acheter une canne à pêche pour mon grand-père, impossible de dire pourquoi, et je n'ai pas envie de savoir pourquoi, de toute façon, c'est un souhait, comme si la canne à pêche était mon grand-père ou que mon grand-père était la canne à pêche.

Je me souviens, sur la route que j'empruntais tous les jours pour aller à l'école, je devais traverser un pont de pierre et sur la gauche s'étendait un lac où des vagues ondulaient sans cesse, même les jours sans vent

[...]

Je me souviens, c'était une cour à l'ancienne, d'un style assez recherché, avec un mur écran gravé des caractères " bonheur ", "félicité ", " longévité " et " joie ", il y avait aussi un " Père longévité " dont la moitié du crâne était effacée, appuyé sur une canne à tête de dragon en partie effacée elle aussi ${ }^{7}$

[...]

Pour Genette, la particularité essentielle du monologue intérieur est l'absence de frontière entre le récit narratif et le discours du personnage:

7. Ibid., p. 56-64. 
Le lecteur se trouverait installé dès les premières lignes dans la pensée du personnage principal, et c'est le déroulement ininterrompu de cette pensée qui, se substituant complètement à la forme usuelle du récit, nous apprendrait ce que fait le personnage et ce qui lui arrive. On a peut-être reconnu dans cette description celle que faisait Joyce des Lauriers sont coupés d'Édouard Dujardin, c'est-à-dire la définition la plus juste de ce que l'on a assez malencontreusement baptisé le " monologue intérieur ", et qu'il vaudrait mieux nommer discours immédiat: puisque l'essentiel, comme il n'a pas échappé à Joyce, n'est pas qu'il soit intérieur mais qu'il soit d'emblée (" dès les premières lignes ») émancipé de tout patronage narratif, qu'il occupe d'entrée de jeu le devant de la " scène ${ }^{8}$ ".

Chez les romanciers du courant de conscience, le monologue intérieur est extériorisé de façon à ce qu’on puisse évacuer toute caractérisation descriptive, de l'extérieur, du sujet pensant (il croit, il pense, il se rappelle, etc.).

C'est en quelque sorte le contraire chez Gao: il répète intentionnellement « je me souviens » pour marquer constamment qu'on est dans le mouvement mental de « je ». Dans les ouvrages relevant du courant de conscience, les monologues intérieurs (discours immédiat) s’insèrent dans les dialogues extérieurs, alors que dans les ouvrages du courant de langage, ce sont les dialogues extérieurs qui s’insèrent dans les monologues intérieurs. Comparons ces paragraphes des Lauriers sont coupés et d'Une Canne à pêche pour mon grand-père:

— « C’est vous? Vous arrivez justement; vous savez qu'à six heures nous partons. Voulez-vous m'attendre; nous descendrons ensemble. » — « Très bien."

La fenêtre est ouverte; derrière, une cour grise, pleine de lumières; les hauts murs gris, clairs de beau temps; l'heureuse journée. Si gentille a été Léa, quand elle m’a dit — à ce soir; elle avait son joli malin sourire, comme il y a deux mois. En face, à une fenêtre, une servante; elle regarde; voilà qu'elle rougit; pourquoi? elle se retire.

— « Me voici. »

C'est Lucien Chavainne. Il a pris sa canne; il ouvre la porte; nous sortons. Les deux, nous descendons l'escalier. Lui:

— « Vous avez votre chapeau rond..."

— «Oui."

8. GenetTE, G., Figures III, Paris, Seuil, 1972, p. 193. 
Il me parle d'un ton blâmeur. Pourquoi ne mettrais-je pas un chapeau rond? Ce garçon croit que l'élégance est à ces futilités. La loge du concierge; vide constamment; bizarre maison. Chavainne va-t-il au moins un peu m'accompagner? À ne vouloir jamais allonger son chemin, il est si ennuyeux. Nous arrivons dans la rue; une voiture à la porte; le soleil éclaire encore, comme en flammes, les façades; la tour Saint-Jacques, devant nous; vers la place du Châtelet nous allons ${ }^{9}$.

L'alternance des monologues intérieurs et des dialogues extérieurs construit dans Les Lauriers sont coupés le rythme principal par lequel le lecteur entre facilement dans le monologue du héros et en éprouve les sensations, grâce à l'effacement de la distance, du quant à soi d'un sujet pensant vu " de l'extérieur ". Dans Une Canne à pêche pour mon grand-père, c'est l'inverse: le monologue intérieur remplit presque seul la nouvelle. Il n'y a qu'un seul dialogue entre « je » et un personnage anonyme:

Soudain une idée me vint à esprit, j'ai repensé au temple du Seigneur Guan, sur la route qui menait au cinéma où ma mère m'emmenait parfois, dans la direction opposée à celle de l'école, on empruntait une ruelle appelée la ruelle du Temple du Seigneur Guan, si je retrouvais ce temple, je n'aurais aucune difficulté à retrouver notre maison. Je me renseignai donc à son sujet.

Ah bon, vous cherchez le temple du Seigneur Guan? A quel numéro? Voilà qui prouvait que ce temple existait. J'avais rencontré un homme chaleureux qui s'enquérait même du numéro que je cherchais, mais je n'ai pas réagi sur-le-champ et je ne me souvenais plus de ce numéro, je n’ai fait que bredouiller: Je voulais juste savoir si cet endroit existait encore. Comment voulez-vous qu'un endroit disparaisse? Qui cherchez-vous? Quelle famille? Il m'interrogeait d'une manière de plus en plus précise, est-ce qu'il me prenait pour un Chinois d'outre-mer en quête de ses racines? ou pour un fils prodigue qui avait coupé avec son pays natal? Je devais m'expliquer: ma famille louait une maison à l'époque, elle ne lui appartenait pas, et comment s'appelait le propriétaire? Tout ce que je savais, c'est Zaowa, bien sûr je ne pouvais pas lui dire les choses comme ça, en me voyant bredouiller, son visage s'est renfrogné et son regard chaleureux s'est glacé d'un seul coup. Il m’a dévisagé de la tête aux pieds, se demandant s'il devait me signaler à la police.

9. DuJARDIN, É., Les Lauriers sont coupés, Paris, Flammarion, 2001 ; p. 3-4. 
Si vous cherchez le numéro un, avancez tout droit et vous y serez, au niveau de la première ruelle à votre droite, au sud de l'avenue, si vous cherchez le numéro trente-sept, passez par là et prenez à une centaine de pas d'ici la deuxième ruelle, vous en passez encore une, vous continuez tout droit et à votre gauche, sur le côté nord de l'avenue, vous y serez, je m'empressai de le remercier, puis je sentis dans mon dos son regard me transpercer ${ }^{10}$.

Si dans Les Lauriers sont coupés, le monologue intérieur se transforme en discours immédiat par un effet d'extériorisation, dans Une Canne à pêche pour mon grand-père, le dialogue extérieur est intériorisé dans le monologue intérieur de «je » : ce n’est pas seulement parce que les signes de ponctuation pour indiquer le dialogue sont supprimés, mais aussi parce que ce qui dialogue vraiment avec l'homme, n'est pas la parole de «je », mais son monologue intérieur. La forme de la phrase l'implique: chaque paragraphe est constitué par une seule phrase où le sens coule sans être entravé à travers les mots et les locutions, sans que la parole de l'homme et le monologue intérieur de "je " puissent l'empêcher. En effet, puisque le courant de langage ne se déroule plus dans un monde extérieur ou entre le monde intérieur et le monde extérieur, mais uniquement dans le monde intérieur, il marque plutôt l'idée essentielle chez Gao que l'homme pénètre plus profondément dans son propre monde lorsqu'il commence à se parler: "On peut dire que se parler à soi-même constitue le point de départ de la littérature, communiquer au moyen du langage vient en second ${ }^{11}$. " On trouvera évidemment ce style encore développé dans les récits de «tu »/« elle » de La Montagne de l'âme et ceux de « tu »/« il » du Livre d'un homme seul.

Les « je me souviens " amènent le lecteur à aller de plus en plus près du fond de sa mémoire en s'exprimant dans un langage « libre » qui traverse aisément le souvenir et le présent, le réel et le rêve/l'imagination:

10. GAO, X. J. Une Canne à pêche pour mon grand-père, op, cit., p. 65-66.

11. GAO, X. J., «La Raison d'être de la littérature », dans Le Témoignage de la littérature, op. cit., p. 113. 
Aller dans les environs $!^{12} \mathrm{Au}$ bord de la rivière où mon grand-père m'emmenait... pêcher? Je me souviens, mon grand-père m'avait emmené au bord d'une rivière, avions-nous pris du poisson, je ne sais plus, mais je me souviens, j'avais un grand-père, j'ai eu une enfance, quand ma mère me donnait mon bain au milieu de la cour et que j'étais nu comme un ver, je me sentis très mal à l'aise, j'ai recherché aussi la maison où j'avais vécu quand j'étais petit, je me souviens encore, en pleine nuit, je m'étais levé pour partir à la chasse avec quelqu'un qui n'était pas mon grand-père, nous avions marché toute la journée et nous avions tué un chat sauvage que nous avions pris pour un renard et ça m'a rappelé un poème dont le héros a le corps hérissé de couteaux de chasse qui cliquettent en s'entrechoquant, une libellule sans queue fait du surplace en battant des ailes, dans les yeux des critiques poussent des épines et leur menton est large, j'ai envie d'écrire un roman d'une profondeur telle que les mouches s'y noieraient, et ensuite, j'ai vu mon grand-père, assis sur un petit tabouret, le dos courbé, fumant sa pipe, et je l'ai appelé, grand-père, il n'a pas entendu, je suis allé près de lui et je l'ai appelé une nouvelle fois, il s'est enfin retourné ${ }^{13}[\ldots]$
到郊外去！到我老 爷曾经带我去过的郊外河 边上去一一钓鱼? 我记 得我老爷带我到河边去 过，钓没钓上鱼我可记不 清了, 可我记得我有个老 爷, 也有过童年, 童年我 妈给我在院子里光屁股洗 澡的时候, 我就周身不自 在, 我也找寻过我小时候 住过的房子, 我也还记得 有一次半夜里就起来跟人 去打猎，跟的并不是我老 爷, 跑了一整天, 打死 了一头野猫, 被当成了狐 狸, 我又想起一首诗, 诗 中的那我，浑身披挂着叮 当作响的猎刀，一只没有 尾巴的蜻蜓, 扑打着翅膀 在原地旋转, 批评家的眼 睛里长着倒刺, 还有一个 很宽的下巴，我想写一篇 大有深意的小说, 深深的 淹得死苍蝇, 后来, 就看 到了我老爷, 蹲在一张小 板発上, 躬着背吧嗒吧嗒 在抽烟, 老爷, 我就叫他 了，他没有听见，我到他 跟前又叫了一声他老爷, 他这才转过身来 $[. . .]^{14}$

Lorsque Genette indique que le discours immédiat se substitue effectivement au monologue intérieur, il vise la synchronisation

12. Nous modifions la traduction de « Je dois aller dans les environs » en « Aller dans les environs » selon le texte original : “到郊外去! ".Voir GAO, X. J., 《高行健短篇小说集 》 (Gao Xingjian Duanpian xiaoshuoji), Taibei, Lianhe Wenxue, 2008, p. 300.

13. GAO, X. J., Une Canne à pêche pour mon grand-père, op, cit., p. 68-69.

14. GAO, X. J., 《高行健短篇小说集 》 (Gao Xingjian Duanpian xiaoshuoji), Taibei, Lianhe Wenxue, 2008, p. 300. 
de la conscience et du discours. Pour capter ce mouvement synchronique, les romanciers du courant de conscience proposent une écriture négligeant le sujet parlant/pensant et appliquant une langue " sautillante ». Ce dont Gao se doute, à partir de sa connaissance des langues occidentales, c'est que cette écriture qui prétend montrer le mouvement de la conscience est entravée en fait par sa structure prédéterminée par le sujet-temps:

À l'instar du courant de conscience, je proposerai la notion de courant de langage. Car la conscience de l'homme échappe à la logique, elle est vivante et bondissante; or les temps, la structure stricte " sujet-prédicat » des langues occidentales la mettent bien mal en point, tandis que le chinois, justement, s'approche au plus près des caractéristiques de son activité 15 .

Rappelons que cette nouvelle est écrite en chinois et que le courant de langage dont Gao parle est exprimé en chinois; cela peut sans doute expliquer pourquoi Gao n'a jamais écrit de roman ou de nouvelle en français. En effet,

La langue chinoise ne distingue pas clairement le temps, on peut très bien prendre le passé, le présent et le futur, le souvenir et l'imagination, le sentiment et la pensée, le réel, le possible et l'illusoire, sans qu'ils soient marqués grammaticalement, pour les transformer en un style direct évoquant des choses qui se passent au même instant. Cela se rapproche davantage encore du processus psychologique, qui dépasse le point de vue réel de l'espace et du temps, et donne à la langue un charme très spécial qui me fascine. Mais cela a aussi constitué une difficulté au moment de traduire ce roman [La Montagne de l'âme] en français. J'ai discuté de cette question avec le traducteur Noël Dutrait, et j'ai proposé d'utiliser du début à la fin le présent de l'indicatif, mais il a estimé que ce serait maladroit en français. On peut voir la différence entre le chinois et les langues occidentales. Comme j'ai bien pris conscience du désastre qu'a produit sur le chinois moderne l'européanisation du chinois, j'ai abandonné ma proposition malheureuse. J'en ai tiré la conclusion que, quelle que soit la langue, l'innovation et la création doivent respecter la grammaire et les structures propres à cette langue ${ }^{16}$.

15. GAO, X. J. et YANG, L., Visite à Gao Xingjian et Yang Lian. Conversation, op. cit., p. 62.

16. GAO, X. J. «Le chinois moderne et l'écriture littéraire », dans Littérature chinoise, état des lieux et mode d'emploi, op. cit., p. 90-91. 
Gao propose ainsi de suivre la linéarité de la langue afin de ne pas aller à l'encontre de sa grammaire, mais aussi pour ne pas être entravé par le temps linguistique ou logique. En somme, le courant de langage respecte l'ordre du temps pour produire le discours/monologue intérieur au lieu du temps linguistique/réel où le sujet parlant se trouve. À comparer la traduction en français et la version originelle en chinois, on voit bien comment Gao utilise au maximum la souplesse et la légèreté de la structure et du fonctionnement du chinois. Dans la traduction française, « je me souviens", "j'avais ", «j'avais vécu " classent clairement les niveaux temporels qui distinguent les éléments de différentes époques de «je »; dans la version chinoise, les éléments s'accumulent dans une même dimension du langage où le temps est spatialisé. «我 (je)" s'introduit entre les locutions qui n'ont pas de syntaxe mais assurent une juxtaposition de différentes niveaux de sensations et de perceptions en suivant la fluidité de la langue elle-même. C'est ainsi que "je " devient un « tu » dans cette extension de l'espace psychologique

[...] je suis arrivé au bord de la rivière, les grains de sable crissent sous mes pas, on dirait les soupirs de ma grand-mère, elle aimait murmurer sans cesse des phrases inaudibles et si tu lui demandais: grandmère, que dis-tu? elle était perdue, levait la tête et disait au bout d'un moment: ah, tu es rentré de l'école? ou vapeur, quand elle murmurait, il valait mieux ne pas l'arrêter, elle parlait de ce qu'elle faisait jeune fille, mais si tu l'écoutais caché derrière un dossier de chaise, elle semblait toujours parler de quelque chose enfoui, enfoui, enfoui, encore enfoui, enfoui, enfoui, enfoui, ces souvenirs crissent sous tes pieds qui foulent le sable ${ }^{17}$.
我来到了河边沙地上, 脚 下的沙子在悉悉作响, 像是我 姥姥在叹气, 我姥姥就好唠唠 叨叨, 可没有一句听得清楚, 你要故意问她, 姥姥, 你讲什 么? 她就立刻失神, 抬起头 来, 半天才说, 啊, 你下学回 来啦? 或是说, 你饿不饿? 厨 房笼屉里蒸得了白薯, 她唠唠 叨叨的时候, 你最好别打断, 她讲的都是自己做姑娘时的 事，可你要是从椅子背后去偷 听, 她就好像是在说掩盖了掩 盖了掩盖了掩盖了掩盖了什么 都掩盖了什么，这些回忆就都 在你脚底下的沙子底下作响。18

17. GAO, X. J. Une Canne à pêche pour mon grand-père, op, cit., p. 69-70.

18. GAO, X. J., «高行健短篇小说集》(Gao Xingjian Duanpian xiaoshuoji), op, cit., p. 301. 
Ce paragraphe semble couler « naturellement » dans le clivage des langages - il ne se déploie ni par le temps ni par la syntaxe ni non plus par le signe, mais par une extension de la perception: du crissement du sable sous les pieds de « je » au murmure de la grandmère, puis encore au crissement du sable sous les pieds de « tu ». La connexion de la dimension de " je " avec celle de " tu " est en effet une extension de " je » dans son souvenir spatial.

Cette extension spatiale ne s'arrête pas là; elle s'étend encore du « je » d'ici et maintenant au « je » de l'enfance où le monologue intérieur apparaît en forme de dialogue extérieur, et où « tu » est comme un spectateur regardant les " je »; ou, pour le dire plus précisément, c'est sous le regard de « tu » que « je » retourne « réellement » dans son enfance:

[...] cette dune qui ressemble à un grand tombeau, avec le crissement menaçant du sable semble dire qu'il va tout envahir, il a déjà submergé les berges de la rivière, il va ensevelir la ville, ensevelir mes/tes souvenirs d'enfance, il n'a pas de bonnes intentions, je ne comprends pas pourquoi mon grand-père reste là sans s'enfuir, je pense qu'il faut vite partir, j'ai vu la dune de sable s'élever en face, et sous le soleil brûlant est apparu un petit enfant fesses nues - moi, à l'époque -, mon grand-père s'est alors levé, les rides sur son visage se sont détendues, il a pris le fin poignet de l'enfant nu que j'étais, mon grand-père portait un pantalon ample noué à la taille, et moi, petit garçon nu comme un ver, je le suivais en sautillant.

Il y a des lièvres?

Hum.

Noiraud vient avec nous?

[...]

Grand-père, j'en peux plus.

Serre les dents et marche.

Grand-père, elles sont tombées, mes dents.

Sale garnement, debout!

Mon grand-père s'accroupit et le petit être tout nu grimpa sur son dos, le grand-père titubait en avançant lentement à grands pas sur le sable, avec cet enfant cul nu sur le dos qui continuait à pousser des cris en agitant les jambes, chevauchant les épaules du grand-père, le fouettant comme il aurait fouetté un cheval, et tu as regardé longtemps, très longtemps la silhouette de ton grand-père disparaître peu à peu ${ }^{19}[\ldots]$

19. GAO, X. J. Une Canne à pêche pour mon grand-père, op, cit., p. 71-74. 
Si le courant de conscience sert à révéler le mouvement conscience/inconscient et à s'émanciper d'une langue narrative, le courant de langage vise lui retourner vers le langage lui-même et à chercher sa potentialité dans l'exploration de la sensation.

\section{Une autre « logique " pour employer les pronoms personnels: comment la subjectivité fluide émerge dans le langage}

Dans ses Problèmes de linguistique générale, Benveniste montre comment le langage permet de mettre au jour la subjectivité:

C'est dans et par le langage que l'homme se constitue comme sujet; parce que le langage seul fonde en réalité, dans $s a$ réalité qui est celle de l'être, le concept d'" ego ». La subjectivité dont nous traitons ici est la capacité du locuteur à se poser comme « sujet ». Elle se définit, non pas par le sentiment que chacun éprouve d'être lui-même (ce sentiment, dans la mesure où l'on peut en faire état, n'est qu'un reflet), mais comme l'unité psychique qui transcende la totalité des expériences vécues qu'elle assemble, et qui assure la permanence de la conscience. Or nous tenons que cette "subjectivité ", qu'on la pose en phénoménologie ou en psychologie, comme on voudra, n'est que l'émergence dans l'être d'une propriété fondamentale du langage. Est " ego " qui dit " ego ». Nous trouvons là le fondement de la "subjectivité ", qui se détermine par le statut linguistique de la « personne $\mathrm{e}^{20}$ ».

Autrement dit, le langage qui fait que la subjectivité émerge est bien ce langage " mis en action, et nécessairement entre partenaires ${ }^{21}$ " que Benveniste appelle "discours ». Les " partenaires ", selon l'interprétation de Jean-Claude Coquet, sont « le temps présent et mettant en présence des personnes » où l'acte de langage s'effectue $^{22}$. Ainsi Benveniste conclut: "il est donc vrai à la lettre que le fondement de la subjectivité est dans l'exercice de la langue ${ }^{23}$ ", ou, plus précisément: "le langage est donc la possibilité de la

20. BENVENISTE, É., Problèmes de linguistique générale I, Paris, Gallimard, 1966, p. 259-260.

21. Ibid., p. 258.

22. COQUET, J.-C., «Temporalité et phénoménologie du langage, sur quelques problèmes posés par la phénoménologie à la linguistique et à la sémiotique », dans Sémiotique, décembre 1993, n 5, p. 10.

23. BenVEnISTE, É., Problèmes de linguistique générale I, op. cit., p. 262. 
subjectivité, du fait qu'il contient toujours les formes linguistiques appropriées à son expression, et le discours provoque l'émergence de la subjectivité, du fait qu'il consiste en instances discrètes ${ }^{24}$ ".

Lorsque Gao propose son courant de langage en remplaçant " conscience " par "langage ", il révèle déjà sa pensée. Où la subjectivité se trouve-t-elle? Il convient de réexaminer le sujet parlant représenté par les pronoms personnels, dont le système de désignation se pose en référence dans les langues occidentales mais ne s'y identifie pas vraiment. Remarquons que dans ses nouvelles et ses romans, notamment à partir des récits inclus dans Une Canne à pêche pour mon grand-père, l'emploi des pronoms personnels est indispensable et se développe encore dans ses pièces de théâtre (sauf dans celles qui sont des épopées: L'Homme sauvage, Chronique du Classique des montagnes et des mers, La Neige en août, etc.) où les rôles sont exprimés par un " je " mais aussi un " tu " ou un " elle/ il ». Le remplacement des noms propres des personnages par des pronoms personnels efface l'identité personnelle et l'image du rôle concret mais rattache étroitement la subjectivité à la langue: à la différence des noms propres, les pronoms personnels ne se réfèrent pas à un individu certain — réel ou fictif - mais seulement à ce dont ils parlent ou ce qu'ils expriment. Mais, en ce sens, à quoi les pronoms personnels se rapportent-ils, chez Gao, préalablement à leur position dans le langage? Représentent-ils une subjectivité, en phase avec leur statut linguistique et introuvable ailleurs que dans le langage?

Selon Benveniste, les pronoms personnels «sont le premier point d'appui pour cette mise au jour de la subjectivité dans le langage ${ }^{25} »$ :

Il n’y pas de concept « je » englobant tous les je qui s'énoncent à tout instant dans les bouches de tous les locuteurs, au sens où il y a un concept " arbre » auquel se ramènent tous les emplois individuels de arbre $^{26}$.

[Car ce "je »] se réfère à " quelque chose de très singulier, qui est exclusivement linguistique: je se réfère à l'acte de discours individuel où il est prononcé, et il en désigne le locuteur ${ }^{27}$.

24. Ibid., p. 263.

25. Ibid., p. 262.

26. Ibid., p. 261.

27. Ibid. 
Le langage est ainsi organisé qu'il permet à chaque locuteur de s'approprier la langue entière en se désignant comme $j e^{28}$.

Benveniste donne encore cette définition de je: "l'individu qui énonce la présente instance de discours contenant l'instance linguistique $j e^{29} "$. Par conséquent, en introduisant la situation d'« allocution ", Benveniste obtient " une définition symétrique pour $t u$, comme l'individu allocuté dans la présente instance de discours contenant l'instance linguistique ${ }^{30}$ ".

$\mathrm{Si}$ « je » implique effectivement une « personne-je », pour « tu » il s'agit d'une « personne non-je » :

Au couple je/tu appartient en propre une corrélation spéciale, que nous appellerons, faute de mieux, corrélation de subjectivité. Ce qui différencie " je » de «tu », c'est d'abord le fait d'être, dans le cas de « je », intérieur à l'énoncé et extérieur à « tu », mais extérieur d’une manière qui ne supprime pas la réalité humaine du dialogue ${ }^{31}$.

Quant au pronom de la troisième personne, Benveniste le considère comme "non-personne ", car il ne possède ni l'une ni l'autre des deux particularités de « je »/« tu »:

En effet une caractéristique des personnes « je » et « tu » est leur unicité spécifique: le « je » qui énonce, le «tu» auquel « je » s’adresse sont chaque fois uniques. Mais « il » peut être une infinité de sujets — ou aucun. [...] Une seconde caractéristique est que « je » et «tu » sont inversibles: celui que "je » définis par " tu » se pense et peut s'inverser en " je », et " je " (moi) devient un « tu ». Aucune relation pareille n'est possible entre l'une de ces deux personnes et «il ", puisque « il » en soi ne désigne spécifiquement rien ni personne ${ }^{32}$.

La Modification de Michel Butor vient confirmer cette définition des positions linguistiques des pronoms personnels chez Benveniste. La voix du narrateur « tu » qui fait du roman un long monologue, ne supprime pourtant pas l'existence d'un « je » invisible et marque justement son absence en impliquant leur relation

28. Ibid., p. 262.

29. Ibid.,

30. Ibid., p. 253.

31. Ibid., p. 232.

32. Ibid., p. 230. 
réversible. Benveniste indique aussi que le monologue est un dialogue intériorisé:

Formulé en langage intérieur, entre un moi locuteur et un moi écouteur. Parfois « le moi locuteur est seul à parler; le moi écouteur reste néanmoins présent; sa présence est nécessaire et suffisante pour rendre signifiante l'énonciation du moi locuteur. Parfois aussi le moi écouteur intervient par une objection, une question, un doute, une insulte [...] Tantôt le moi écouteur se substitue au moi locuteur et s'énonce donc comme "première personne » [...] Tantôt le moi écouteur interpelle à la « deuxième personne » le moi locuteur ${ }^{33}[\ldots]$

De même, dans La Modification, lorsque le narrateur se parle en employant «tu ", c'est le moi écouteur qui parle au moi locuteur et le " je ", qui se place en moi écouteur, reste muet, invisible, comme la polarité potentielle de «tu ». Tandis que pour «elle», par son caractère de "non-personne " qui peut être " une infinité de sujets ", l'auteur donne enfin et aussi obligatoirement des noms propres pour distinguer les femmes autour de " tu »: Cécile pour désigner son amante et Henriette pour sa femme. En d'autres termes, ce qui compose la polarité avec « tu » narrateur, est le « je » du narrateur potentiel, au lieu du « elle » de Cécile, Henriette.

Bien que sa narratologie des pronoms personnels soit inspirée fortement par La Modification, il semble que Gao donne une autre définition de la « logique » des pronoms personnels. Il s'explique:

Ici, pourquoi ne pas parodier le mot de Descartes en déclarant pour ce qui concerne l'écrivain: « Je m'exprime donc je suis »? " Je », l'écrivain, ce peut être l'écrivain lui-même ou le narrateur, ou encore un personnage du livre, ce peut être « il », mais aussi « tu », le narrateur sujet (叙述者主体) peut passer d'un à trois. La reconnaissance de la personne sujet (主语人称的确定) est le point de départ de l'expression de ses perceptions dans ce processus de recherche d'un mode de narration original ${ }^{34}$.

On en voit bien des exemples dans La Montagne de l'âme où « je », « tu » et « elle » constituent un long monologue à travers tout ce roman; de même dans Le Livre d'un homme seul où « tu » et « il »

33. BENVENISTE, É., Problèmes de linguistique générale II, Gallimard, 1974, p. 85.

34. GAO, X. J., "La raison d'être la littérature », dans Le Témoignage de la littérature, op. cit., p. 127-128; nous modifions la traduction de 感知 en perceptions. 
- les deux moi dialoguent. Remarquons que si Benveniste parle du jeu de substitution entre «tu » et « je » dans le monologue, il n'a jamais pris en compte la troisième personne « il/elle » ni dans le dialogue ni dans le monologue.

Comment comprendre ce rapport réversible entre " je ", " tu " et « il/elle »? De quoi s'agit-il dans la position des pronoms personnels chez Gao? Certes, la langue chinoise donne tout d'abord à Gao la possibilité technique de faire une expérimentation linguistique, puisqu'elle ne conjugue les verbes ni selon le sujet ni selon le temps. Lorsque la fameuse phrase " je est un autre " de Rimbaud est traduite en chinois, elle ne provoque aucun « dérangement » au niveau grammatical.

Or, l'échange libre entre les pronoms personnels chez Gao n'est pas sans origine: leur utilisation n'est pas fixée dans la langue chinoise avant le $\mathrm{xx}^{\mathrm{e}}$ siècle. Par exemple, dans une phrase qui se trouve dans Shiji (“史记 ”, Mémoires historiques): 《孝惠为人仁 弱, 高祖以为不类我, 常欲废太子, 立戚姬子如意, 如意类 我 ${ }^{35}$ (Puisque [le dauphin] Xiaohui eut caractère doux et délicat, Gaozu [l'empereur] crut qu'il ne lui ressemblait pas, voulait souvent abroger son droit de dauphin, et le fit remplacer par Ruyi, le fils de QIji [sa favorite], qui lui ressemblait), 高祖以为不类我 et 如意类我 utilisent tous 我 (je) qui accorde grammaticalement le narrateur au « je » de l'empereur. La phrase sera, si nous la traduisons littéralement en français: « [...] Gaozu [l’empereur] crut qu'il ne me ressemblait pas [...] Ruyi me ressemblait ». Il ne s'agit pas d'une métamorphose du discours direct mais d'un remplacement du sujet qui raconte: le narrateur cède sa place de «sujet racontant " à l'empereur Gaozu qui est en effet le centre de la phrase. On voit bien qu'il n'y a pas de position claire ni pour le narrateur, ni pour la conscience du narrateur en tant que sujet racontant à travers cette utilisation des pronoms personnels. Pour tout dire, les pronoms personnels employés dans la langue chinoise ne distinguent pas sujet parlant et objet parlé de la même façon que les langues occidentales.

Mais de plus, les pronoms personnels ne s'attachent pas étroitement au temps. L'utilisation des pronoms personnels chez Butor

35. SIMA, Q., «史记・吕太后本纪» (Shiij), Changsha, Yuelu chubanshe, 1988, p. 110. 
respecte le même principe que chez Benveniste: la production du discours s'opère avec ses propriétés indissociables, que celui-ci appelle " les instances de discours »: " c'est-à-dire les actes discrets et chaque fois uniques par lesquels la langue est actualisée en parole par un locuteur ${ }^{36}$ ». Jean-Claude Coquet l'explique en le liant d'emblée au temps: ce qui " concerne le mot lui-même [instance]... c'est-à-dire comme le temps ancré (in-) dans le maintenant ", mais " ancré ne veut pas dire immobile, mais qui a son assise dans le réel ${ }^{37}$ ». En ce sens, « je ", l’« individu qui énonce la présente instance de discours » est en effet la polarité temporelle de « tu » — « individu allocuté dans la présente instance de discours ", autrement dit, " je » et « tu » sont un couple linguistique synchrone.

Dans Une Canne à pêche pour mon grand-père, " tu " apparaît dans l'extension du souvenir spatial de "je ». Le lien spatial se présente comme une évidence entre les pronoms personnels chez Gao en marquant leur position atemporelle ou non entravée par le temps. Dans La Montagne de l'âme, " tu ", dérivant de " je " qui voyage le long d'un axe spatial, pénètre un monde spirituel le long de l'axe du temps psychologique au lieu du temps réel, et rencontre « je " dans un espace singulier: " et plus tu vas loin, plus tu te rapproches jusqu'à ce que, inévitablement, il devienne impossible de nous dissocier ${ }^{38}$ ". Tandis que dans Le Livre d'un homme seul, " tu " et « il ", qui sont respectivement dans le présent et dans le passé, dialoguent à travers le temps: il semble qu'ils se juxtaposent spatialement en marquant les deux époques différentes mais sans s'accorder ni avec les temps linguistiques (en chinois, il n'y a pas de conjugaison du temps) ni avec le temps où ils se trouvent.

Ainsi chez Gao, la position de "je " est ambiguë: "je " est ce qui est le plus proche, parmi les deux autres pronoms personnels,

36. BENVENISTE, É., Problèmes de linguistique générale I, Gallimard, op. cit., p. 251.

37. Coquet formule trois remarques à propos cette notion : le temps du maintenant; deux versants substantiel et formel qui composent «les instances »; et « ancré » a son assise dans le réel. Voir «Temporalité et phénoménologie du langage, sur quelques problèmes posés par la phénoménologie à la linguistique et à la sémiotique », dans Sémiotique, $n^{\circ} 5$, décembre 1993, p. 10.

38. GAO, X. J., La Montagne de l'âme, op. cit., p. 422. 
de la position linguistique comme l'entend Benveniste, en tant que sujet énonçant; mais en même temps "je " se réfère à la psychologie du moi qui se fonde sur le processus ouvert et permanent de perception et d'expérience. "Je " s'ouvre ainsi aux autres pronoms personnels au niveau psychologique et perspectif lorsque «je " prend la place du « tu » ou « il/elle » afin d'observer le moi — dans la version chinoise, il convient d'ajouter encore: « je » s'ouvre aussi au niveau grammatical. Cependant, ce « je » qui est plus assuré, au sens linguistique, de représenter la subjectivité du moi, se retire peu à peu de sa position de sujet parlant: la répétition de " je me souviens que... » dans Une Canne à pêche pour mon grand-père réapparaît sous les formes " tu dis que... ", " elle/il dit que... » ou " tu dis que tu... ", " elle/il dit qu'elle/il » dans les romans suivants et les pièces de théâtre tels qu'Au bord de la vie, Dialoguer-interloquer, Le Somnambule, Le Quêteur de la mort, Quatre Quatuors pour un weekend, Ballade nocturne...; de même " je " de La Montagne de l'âme a disparu complètement dans Le Livre d'un homme seul. Il semble que Gao évite de rattacher la subjectivité au sujet parlant qui est " déterminé " par sa position linguistique; il prend plutôt " tu » et " il/elle », grâce à leurs fonctionnements moins assurés que celui de " je ». Car la subjectivité, selon lui, n'est pas une question de « qui suis-je » ni celle d'" un certain lieu où (singulier) » je me situe, mais celle des "lieux où (plusieurs, atemporels)" je me trouve sous des angles variés.

\section{Premier acte de Dialoguer-interloquer: le langage à la fois fermé et ouvert}

Mon langage est-il le produit du moi? Si oui, ma parole n'est-elle que ce que je savais avant? Et est-ce la même chose de parler avec l'autre et de parler avec moi-même? Dans ce sens, le dialogue entre moi et l'autre n'est-il qu'une sorte d'" échange » des productions linguistiques, et ce moi et l'autre ne s'enferment-ils pas toujours dans leurs propres langages?

Remarquons la manière singulière d' " écrire " qui inspire le style $\mathrm{du}$ « courant de langage » chez Gao. Il s'exprime oralement en enregistrant, puis en transférant en écriture: 
J'enregistre au magnétophone mon premier jet puis, quand je travaille le manuscrit, je le relis mentalement. Tout langage vivant possède un rythme propre, et le tester par l'ouïe est un bon procédé39.

Parlant à voix haute et s'écoutant attentivement, Gao a redécouvert son propre langage:

On doit prêter attention à la langue qui sort de sa plume, on doit la prononcer en soi-même, comme l'instrumentiste ou le chanteur qui écoute sa propre voix. La langue, animée d'une âme, devient alors vivante et poétique ${ }^{40}$.

Le langage ne s'incarne pas seulement dans l'écriture sur papier ou dans le sens: il est vivant, aussi, dans la relation avec l'homme et aussi avec lui-même, il "n'est pas seulement le vecteur de concepts et de points de vue, il touche en même temps la sensation et l'intuition $^{41}$ "; " dans le noir, je me concentre pleinement et je trouve le ton. On peut dire alors que la langue parle d'elle-même ${ }^{42}$. "

C'est sans doute la raison pour laquelle Gao s'éloigne de l'influence structuraliste et ne se place pas non plus dans le sillon de la sémantique, car le langage pour lui n'est pas un objet où l'on trouvera le reflet du sujet, mais un sujet:

Les signes et l'informatique ne pourront remplacer le langage des êtres vivants [...] Si l'homme a besoin du langage, ce n'est pas seulement pour communiquer du sens, c'est en même temps pour écouter et reconnaître sa propre existence ${ }^{43}$.

Cette manière de "parler-écouter-reconnaître ", ne permet donc pas seulement à l'auteur de s'incruster, à la fois en tant que narrateur et auditeur, dans ses propres ouvrages; elle permet aussi

39. GAO, X. J., "Le chinois moderne et l'écriture littéraire », dans Le Témoignage de la littérature, op. cit., p. 87. Gao donne lui-même ailleurs un exemple : la pièce de théâtre Signal absolu, a été achevée en 36 heures, dans le temps durant lequel il parlait tout seul devant son magnétophone.

40. Ibid., p. 105.

41. GAO, X. J., "La Raison d'être de la littérature », dans Le Témoignage de la littérature, op. cit., p. 127.

42. GAO, X. J., Entretien avec Jin Siyan et Wang Yipei, dans Dialogue transculturel, Nice, Alliage, n², 2001, p. 102; cité par Jin Siyan dans L'Écriture subjective dans la littérature chinoise contemporaine: devenir je, op. cit., p. 202.

43. Ibid., p. 127 ; nous remplaçons « codes » par « signes » par fidélité au texte chinois : “符号和信息无法取代活人的语言”。 
au langage lui-même, pour autant qu'il est une de nos fonctions (comme la vision), de se présenter comme un autre moi qui reconstruit son rapport avec le moi; c'est en ce sens que la "subjectivité " émerge dans le langage. C'est aussi pourquoi les ouvrages de Gao sont toujours marqués par la parole: monologue, dialogue, interrogation, et ses nouvelles et romans se prolongent aussi en pièces de théâtre.

La pièce S'abriter de la pluie ( «躲雨 》) est une adaptation directe de la nouvelle La Pluie, la Neige et l'Autre ( 雨、雪及其他»), sans beaucoup de modifications. S'abriter de la pluie est un long dialogue entre deux jeunes filles qui échangent leurs impressions au sujet d'événements climatiques comme la pluie et la neige, des impressions qui reflètent leur vision de la vie, en s'abritant de la pluie dans une baraque de chantier. Il y a là un vieil homme assis de l'autre côté de cette baraque pour échapper lui aussi à la pluie. Il écoute leur dialogue sans prononcer un mot, sans être perçu par elles. Tandis que dans La Pluie, la Neige et l'Autre, c'est un « tu » qui est l'auditeur de ces deux filles.

S'abriter de la pluie qui présente le même sujet et la même structure que La Pluie, la Neige et l'Autre, expose aussi une situation semblable: le dialogue n'est pas seulement entre les deux filles mais aussi en dehors d'elles. Les deux filles et le vieil homme sont comme deux parties complémentaires l'une de l'autre: les deux filles qui s'incarnent dans une voix douce et une voix claire; le vieil homme qui est présent sur la scène mais sans voix. Il écoute, pas seulement le sens mais aussi la sonorité. Le vieil homme communique avec le dialogue des filles par les mouvements de son corps (se lever, toussailler, s'asseoir, hocher la tête, mettre ses lunettes,...) qui construisent un autre dialogue: avec le langage lui-même. Autrement dit, il y a deux dialogues parallèles: le dialogue entre les deux filles et le dialogue entre le langage (la sonorité et le sens) et le geste corporel. Cette structure parallèle est remarquable et récurrente chez Gao: elle apparaît dans les deux nouvelles La Pluie, la Neige et l'Autre (1982) et Dans un parc (1983), puis dans deux pièces de théâtre S'abriter de la pluie (1984) et Dialoguer-interloquer (1992). 
La troisième personne représentée par le vieil homme dans S'abriter de la pluie et le " tu " dans La Pluie, la Neige et l'Autre révèle justement, à travers la communication qui s'opère en dehors des deux filles, une dimension du langage qui est en dehors du langage. Gao la pousse encore dans Dialoguer-interloquer: il s'agit d'intérioriser et extérioriser à la fois cette troisième personne.

Dialoguer-interloquer est une pièce en deux actes; le premier est un dialogue entre une femme et un homme après leur " one night sex ». Ce dialogue ne les mène pas à la communication, mais au conflit. Ils ont beau essayer de parler "sincèrement ", tout de suite le langage provoque la violence. Ils jouent un jeu de mort: la femme tue l'homme et ensuite le fantôme de l'homme tue la femme. Au deuxième acte, les deux fantômes recommencent à parler dans le style du gong'an ${ }^{44}$ (公案) et leur langage s'ouvre à une certaine liberté du sens. Un moine chán est présent sur scène, durant les deux actes. À aucun moment il ne communique par la parole avec la femme et l'homme, mais ses gestes agissent sur les deux personnages et les paroles de ces derniers l'atteignent.

L'homme: Alors, c'est fini?

La femme: C'est bien, non?

L'homme: Tu es souvent comme ça?

La femme: Comme ça?

L'homme: Avec les hommes...

La femme: Bien sûr, tu n'es pas le premier.

(L'homme est étonné; il rit)

La femme: Vous êtes tous pareils.

L'homme: (content) Tu dis nous...

La femme: Je dis vous, les hommes!

L'homme (rectifiant): Les hommes et les femmes!

(Ils rient. La femme s'arrête; l'homme cesse aussi)

L'homme: Qu'est-ce qu'il y a?

La femme: Il n'y a rien.

L'homme: Excuse-moi.

La femme (glacée): Pas besoin d'excuser.

(L'homme s'éloigne. Il enfile le peignoir)

La femme: Je le savais...

44. Gong'an: une brève anecdote ou un court échange entre un maître et son disciple, «absurde», énigmatique ou paradoxal, ne sollicitant pas la logique ordinaire, utilisée dans certaines écoles du bouddhisme chán. 
L'homme: Tu parles de toi, pas de moi.

La femme: Hypocrite!

L'homme: Je t'aime...

(La femme éclate de rire. L'homme rit avec elle. La tête tondue, les yeux baissés, portant une longue robe et des sandales de chanvre, les mains jointes, un moine entre par la droite en psalmodiant: "gloire au Bouddha Amitâbha». Il avance lentement. L'homme et la femme s'arrêtent de rire. Le moine gagne l'avant-droit de la scène, se tourne et présente son dos aux spectateurs. Il s'assied, jambes croisées, et tape sur le poisson de bois. L'homme et la femme se contiennent. Les yeux baissés, ils écoutent attentivement les coups réguliers sur le poisson) La femme (à voix basse): Elle ne comprend pas pourquoi, pourquoi elle a suivi cet homme, mais c'est ainsi, elle l'a suivi, elle est là avec lui...

L'homme à voix basse): Elle le comprend, elle le sait, C'est simple et clair, il y a toujours cette nécessité.

La femme (à voix basse): Non, elle voulait seulement savoir s'il allait se passer... elle savait que c'était possible, sans être une règle absolue...

L'homme (à voix basse): A tout instant, à tout endroit du monde, il se passe quelque chose. À chaque fois, tu joues ta pièce.

La femme (à voix basse): Son empressement, C'est évidemment affecté. Si elle tentait de s'y dérober, tout serait réglét5.

L'homme (à voix basse): À une minute près, pourquoi prendre de grands airs? L'un et l'autre, c'est forcément ainsi.

La femme (à voix basse): Bien sûr, elle l'avait prévu. Dès le début, elle voulait en finir. Mais elle n'avait pas imaginé que ça serait si soudain, si précipité, que ça finirait si vite.

(Le moine tape sur le poisson)

La femme: Assez! Il n’y a pas de quoi exulter.

L'homme: Je n'ai pas dit un mot.

La femme: Mieux vaut que tu te taises ${ }^{46}$.

Lorsque les deux personnages dialoguent, ils se désignent eux-mêmes comme "je " et l'autre comme «tu ". Cette réduction pronominale, l'absence du partage qu'est l'énoncé d'un nom ou d'un prénom trahit un parti-pris: le sujet parlant s'enferme et refuse d'être compris ou d'être expliqué par son interlocuteur. Leur

45. Nous modifions la traduction («Son empressement, tu sais qu'il est affecté. Si elle tentait de s'y dérober, tout serait réglé. ») selon la version chinoise où il n'y a pas « tu » : «他 那份殷勤, 明知道虚情假意, 她要是竭力推脱, 也就了却》。

46. GAO, X. J., Dialoguer-interloquer, op. cit., 2001, p. 6-7. 
" communication " semble s'enclencher par le corps mais bloque dans le dialogue lorsqu'ils se présentent réciproquement en tant que « je ». Autrement dit, leur opposition est signée dès le début par celle des deux pronoms personnels d'emploi symétrique " je " et " tu ». Les coups sur le poisson (voir infra) interrompent leur dialogue et les font se plonger dans leur propre monologue où ils s'adressent respectivement à eux-mêmes comme " elle " et " tu ". Dès lors, " elle » et « tu » s'expriment sous le régime du monologue; mais ils parviennent à se comprendre l'un et l'autre.

Il y a donc une troisième personne qui se tient dans l'en-dehors du dialogue. À la différence de ce qu'on a vu dans S'abriter de la pluie, où le vieil homme qui est un auditeur discret et extérieur fait polarité avec le dialogue des deux filles, dans Dialoguer-interloquer, cette troisième personne est à la fois extériorisée - sous la figure de moine qui se tait - et intériorisée en " tu " de l'homme et en " elle " de la femme qui se distinguent du « je » qu'ils sont pour eux-mêmes.

La répétition de " je dis/parle " traduit un malentendu et une dérive dans le langage chez leur interlocuteur:

L'homme: Tu ne bois vraiment rien?

La femme: Si tu fais pas mélanger quelque chose dans le verre. Il n’y a pas plus désastreux.

L’homme: Ça veut dire, tu l'y as mélangé, tu as eu toute sortes d'expériences.

La femme: Je parle de quelqu'un d'autre qui le glisse dans ton verre que tu bois sans même t'en rendre compte.

L'homme: Tu veux dire, tout à l'heure, au café. Si quelque chose a été mis, en tout cas ça ne l'a pas été par moi.

La femme: Je parle d'une fois, en Inde.

L'homme: quoi qu'il en soit, ici ce n'est pas en Inde.

La femme: Je dis qu'une fois, j'ai fait un voyage en Inde ${ }^{47}$.

Ils dialoguent mais en même temps n'échangent pas: ils ne parlent qu'en dévidant le fil de leur propre trame linguistique: la parole de la femme la reconduit vers l'expérience de son enlèvement en Inde, malgré la diversion apportée par la réplique de l'homme; et l'homme ne croit pas ce que la femme raconte, malgré l'insistance de la femme et la répétition du propos. Il y a « communication » vaine entre les deux

47. Ibid., p. 12; nous modifions quelques points de la traduction. 
sujets parlants. Le sens s'enferme dans le langage, comme le sujet parlant s'enferme dans sa propre parole - le langage comme un objet solide est lancé vers l'interlocuteur, surtout au moment où les deux protagonistes se mettent dans la position polarisée femme/homme et disent pour eux-mêmes « je », et pour leur interlocuteur, « tu »:

La femme (retrouvant ses esprits, elle tourne la tête et le regarde):

Pourquoi?

L'homme: Parce que.

La femme: Tu m’aimes pas écouter?

L’homme: Je t'écoute.

La femme: Que veux-tu écouter?

L'homme: Ce que tu as envie de raconter.

La femme: Et si je raconte que je suis une femme dévergondée?

L'homme: Ça, c'est toi qui le dis.

La femme: Ce n'est pas ce que tu espères, que les femmes soient dévergondées?

L'homme: Au départ, les femmes sont ainsi.

La femme: Voilà bien vos illusions.

L'homme: Les hommes, en fait, le sont aussi.

La femme: Qu'y a-t-il là d'étonnant?

L'homme: La seule différence, ce sont les sexes ${ }^{48}$.

Comme dans la scène précédente, le geste du moine (il enlève sa robe) appelle un " tu " chez l'homme et le motive à communiquer de nouveau avec la femme comme « moi » et «toi »:

(Le moine a retiré sa robe: il est en vêtement court. Il fait un saut périlleux et entre en scène. Il retient sa respiration, se concentre. Il se tient droit comme un piquet)

L'homme (regardant le moine, à voix basse): Tu ne comprends rien aux pensées intimes des femmes. (À voix haute) Histoire intéressante, très intéressante. (Tournant la tête pour regarder la femme) Comment se fait-il qu'il ne t’ait pas tuée?

La femme: pourquoi?

L'homme: Parce que.

La femme: Vous ne savez que posséder la femme, la posséder, la posséder jusqu'à la détruire! (En soupirant) Les hommes sont égoïstes, ils ne pensent qu'à eux.

L'homme: pourquoi chez toi c'est toujours les hommes, les hommes?

La femme: N'en es-tu pas un?

L'homme: Je suis une personne, quelqu'un de concret et vivant.

48. Ibid., p. 16-17. 
La femme: Pourtant tu ne m’as pas considérée comme une personne. Je ne suis pas un instrument qui te permet d'épancher ton désir. Les femmes ne sont pas toutes pareilles.

L'homme: Parce qu'au début on peut considérer qu'on parlait en termes généraux. Ce n’est pas comme maintenant.

La femme: C'est comment maintenant?

L'homme: Maintenant, il s'agit de toi et de moi. D'un homme et une femme, sortis du général, qui se font face et se voient l'un l'autre. Ils sont entrés en relation, je ne parle pas d'une relation physique. Ils ont une certaine compréhension, ils ont des sensations. Ils sont vivants ${ }^{49}$.

Cependant ce " toi » et ce " moi » ne provoquent guère qu'une discussion sur le nom propre: signe d’identité dont ils évident, dévitalisent la signification:

La femme: Maria ou Anna Wang. Que choisis-tu?

L'homme: Lequel est ton vrai nom? Là est la question.

La femme: Si je dis Maria, est-ce vraiment Maria?

L’homme: En voilà une énigme. Pourtant si je t’appelle Anna Wang, tu continues à être toi, sans être Anna Wang, c'est pourquoi tu n'as pas besoin d'y attacher beaucoup d'importance.

La femme: (sèchement) Je n'ai pas envie d'être un prête-nom.

L'homme: Naturellement. Le nom n'est qu'un code; ce n'est pas le signe qui compte. L'essentiel, c'est la véritable personne qui se trouve derrière ce signe. Si tu m'appelles du nom que tu veux, si tu y es habituée, et si un jour il te vient par mégarde un autre nom, je ne crois pas que je t'en tiendrai rigueur.

La femme: Ça ne me tracasse pas non plus. Je ne tiens pas à connaître ton nom; à quoi ça me servirait? Tu n'as pas besoin de te fatiguer à t'inventer un faux nom, qui sera oublié dans un instant. Quand c'est fini, c'est fini.

L'homme: Ça vient juste de commencer, pourquoi ça serait déjà fini? Puisque tu es d'accord! Que les noms n'ont pas d'importance, qu'ils sont encombrants. Ce qui compte, c'est ce qu'il y a entre toi et moi. La femme: Un homme et une femme? Comme c'est drôle ${ }^{50}$ !

En retombant dans le cycle: moi/toi-l'homme/la femme, le dialogue trahit de nouveau l'échec dans l'établissement d'une relation entre « je » et « tu ». Chez les personnages de Gao, l'auto-appellation

49. Ibid., p. 17-18.

50. Ibid., p. 20. 
par « je » signale toujours un retour vers soi, ou une fermeture en soi. Les dialogues entre la femme et l'homme sont pour reprendre les mots de Merleau-Ponty,

fermés sur leurs significations - entre eux des messages qui circulent, mais qui ne portent rien, et qui sont seulement occasion pour chacun de faire attention à ce qu'il savait déjà - finalement, quand l'un parle et que l'autre écoute, des pensées qui se reproduisent l'une l'autre, mais à leur insu, et sans jamais s'affronter -, oui comme le dit Paulhan, cette théorie commune du langage aurait pour conséquence " que tout se passât à la fin entre eux deux comme s'il n'y avait pas eu langage ${ }^{51}$.».

Il semble que ces autres moi, autres que "je " et représentés par le moine, le " tu » de l'homme et le " elle » de la femme soient capables de déchirer une couture, de percer une ouverture dans le langage, et instaurent une vraie communication. Mais la relation qu'ils établissent n'est pas une relation entre moi et l'autre ou entre « je » et « tu »: c'est une relation entre moi et l'autre moi. C'est pourquoi, lorsque la femme et l'homme s'appellent par « tu »/« elle », leur dialogue n'est qu'un parallélisme.

\section{Deuxième acte de Dialoguer-interloquer: où suis-je lorsque je parle?}

Le deuxième acte s'ouvre sur une scène où « il ne reste que deux têtes d'homme et de femme éclairées par les lampes. L'homme et la femme sont étendus dans un coin sombre ${ }^{52}$ ». Les fantômes de la femme et de l'homme dialoguent à nouveau:

La femme: Ne sommes-nous pas déjà morts?

(Ils se concentrent. La tête baissée, ils restent silencieux)

L'homme: On dirait que oui. (Il regarde les deux têtes) Il n’y a que toi et moi. Les gens dehors ne sont pas au courant. Et puis, tu ne vas pas parler, je ne vais pas le faire non plus, personne, absolument personne ne le peut!

[...]

L'homme (jubilant comme un enfant): Ni toi ni moi, nous ne pouvons revenir en arrière! Que tu le veuilles ou non, ahahah, nous ne pou-

51. Merleau-Ponty, M., La Prose du monde, dans Maurice Merleau-Ponty, Euvres, op. cit., p. 1441.

52. Ibid., p. 49 
vons, tels des ombres, que vivre ensemble éternellement! En étant l'un à l'autre nos ombres réciproques.

La femme: Comment cela peut-il te faire plaisir? Te voilà transformé en ombre, en esclave couché aux pieds d'une femme.

L'homme: Ça n'a pas d'importance. L'un à l'autre, l'autre à l'un. De toute façon, aucun de nous ne peut partir. Tu es mon ombre, ou bien je suis la tienne, c'est égal ${ }^{53}$.

C'est une scène assez significative: les corps de la femme et de l'homme n'existent plus, leurs têtes sont coupées, mais leurs conversations continuent malgré tout. Dans un lieu équivoque que «le temps même ignore ", les deux sujets parlants ne peuvent savoir s'ils sont vraiment morts: "Je ne sais pas, je ne sais rien, la mort est-elle préférable à la vie? Je ne m'exprime pas clairement, tout est confus, insaisissable ${ }^{54}$... ».Mais qui est ce « je » en train de parler? Qu'est-ce qu'un « je » qui ne fait référence ni au corps ni à la tête? D’ailleurs les deux « je " sont encore remplacés par « tu » et " elle » lorsqu’ils regardent les têtes séparées d’eux-mêmes:

(Elle regarde la tête de la femme. Elle se traîne jusque devant la tête; son regard est fixe)

L'homme: Que fais-tu?

La femme: Rien.

L'homme (il s'assied): On se croirait en plein cauchemar. (Il regarde aussi une tête, celle de l'homme) C'est... ma tête? Tu crois en la réincarnation?

La femme: Comment?

L'homme: Aux idées sur Samsara. ${ }^{55}$

La femme: Que dis-tu?

L'homme: Rien du tout.

(Ils sont assis en silence, dos à dos)

La femme (avec obstination): Elle demande: que dis-tu?

L'homme (avec lassitude): Tu dis: tu n'as rien dit.

(Ils s'assoient sur leurs talons. La femme approche son visage de la tête de l'homme)

La femme: Elle dit qu'elle t'entend parfaitement parler.

[...]

53. GAO, X. J., Dialoguer-interloquer, op. cit., 2001, p. 51-52.

54. Ibid., p. 52.

55. Nous remplaçons « métempsychose » par « Samsara (轮回) 》, notion bouddhiste, en conformité avec l'original chinois. 
(La femme est assise droite. Elle se couvre le visage de ses mains, et baisse la tête. L'homme regarde la tête de la femme)

L'homme: Alors tu vois une vilaine expression. Tu dis, tu veux parler et tu ne lui dis rien. Tu veux parler, mais même si tu parles, c'est de toi que tu parles, sans rapport avec elle. Et ce prétendu toi, ce qui ne désigne que toi, c'est seulement ton moi. C'est de toi, de ton moi que tu veux parler, qui te tracasse sans fin.

La femme: Elle dit qu'elle redoute le silence. Ce face à face muet est insupportable, il l'étouffe. Elle redoute le silence, bien plus que la mort. La mort se vit plus facilement que ce silence sans paroles.

L'homme: Tu dis toi: c'est juste un monologue.

La femme: Elle dit elle: il ne lui reste qu'un souvenir.

L'homme: tu dis toi: seul le monologue t’apporte du réconfort.

La femme: Elle dit elle: seul le souvenir éveille chez elle de l'illusion. L'homme: Tu dis que ce n'est pas que tu ne veux pas te débarrasser de ton moi. Le problème, c'est que tu monologues toujours, et que ton moi n'en finit pas de s'embrouiller ${ }^{56}$.

À la différence de ce qui se passait au premier acte, le changement des pronoms personnels dans le deuxième acte n’est plus suscité par les gestes du moine, mais par leurs regards qui ne leur appartiennent plus. Les deux sujets parlants se plongent désormais dans un dialogue intériorisé avec eux-mêmes (tu dis toi, elle dit elle); tandis que la « communication " entre la femme et l'homme devient parallèle.

La séparation de la tête et du sujet parlant signifie d'emblée la fêlure entre « je suis » et « je parle ». Si l'on a pu considérer jadis que se dire « je pense » suffisait à garantir qu'on soit et qu'on soit « je, chose pensante ", liée à un corps, donc qu'on soit positionné dans l'espace, là où je suis et pas ailleurs - en revanche, ici chez Gao, c’est le " parler » qui donne à constater qu'il n'est aucun « je » porté par aucune substance, dans aucun lieu propre. Le moi émerge dans le langage, mais le sujet parlant du langage n'est en rien certain. Car le sujet parlant ne se réfère pas à une entité mais à un processus de mutation. Autrement dit, avec le moi il ne s’agit ni de « je » ni de « tu »/« elle », mais de ce qui est entre « je » et « tu »/« elle »: c'est un moi a-ontologique fluide.

À l'opposé d'un moi substantiel, qui reste toujours intégré dans le panorama ontologique dont la structure des langues européennes

56. GAO, X. J., Dialoguer-interloquer, op. cit., 2001, p. 53-54. 
dresse la carte, le moi a-ontologique ne peut avoir d'autre attache qu'une langue qui ne possède pas le verbe "être "; une langue qui n'a jamais manifesté l'intention de construire un système de grammaire semblable à celui de l'Occident. La donnée de référence, ici, c'est l'" entre " que nous avons déjà rencontré chez Gao, cet " entre " qui échappe à la philosophie européenne et tient une place essentielle dans la pensée et l'esthétique chinoises. C'est sur l'« entre » que le moi a-ontologique s'appuie sans avoir à se rattacher ni au sujet parlant, ni au langage qui se focalise sur le « sujet » du discours.

Mais qu'est-ce qu'un langage qui ne se focalise pas sur la " chose " ou l'" être" - mais veut pourtant les atteindre, dans le cas du chinois? François Jullien explique comment «parler » dans la philosophie occidentale vise à dire " quelque chose ": « Les Grecs nous ont légué principalement une chose qui ne se dit complètement que dans leur langue: logos - logos signifiant à la fois parole-discours-définition-argumentation-jugement (susceptible de vrai ou de faux)-ordre et, finalement logique. [...] que "parler " soit " dire " et que dire, devenant transitif, soit " dire quelque chose ", legein $t^{i 7}$ ". Quant à " dire quelque chose ", c'est, " poursuit Aristote de façon quasi synonymique, "signifier quelque chose" (legein $t i=$ semainein $\left.t i^{58}\right)$ "

À l'opposé, dans le taoïsme, notamment chez Laozi et Zhuangzi, il y a un langage consistant à "parler sans parler " (言无言) qui est à l'envers de ce "parler » qui veut dire/signifier quelque chose. Zhuangzi se méfie d'emblée du langage, qui n'est pas capable d'exprimer complètement la chose même: ainsi le Dao n'a pas de nom, la véritable éloquence se passe de mots ${ }^{59}$ (大道不称, 大辩不言). Cette idée a été encore développée par les bouddhistes chinois, qui l'unissent à la pensée chán. Il suffit de penser au fameux paradoxe de l'indicible, dans le bouddhisme chán, selon lequel tathate (真如) ne peut être exprimé par la parole, car si on arrive à le dire, c'est qu'il est faux. C'est aussi pourquoi, dans les histoires du gong'an (公案) de l'école de chán, si la réponse ne correspond pas à la

57. JULLIEN, F., Si parler va sans dire, op. cit., p. 14.

58. Ibid., p. 18.

59. ZHANGZi «庄子集释・齐物论》(Zhuangzi Jishi), op. cit., 2004, p. 83. 
question, c'est tout d'abord pour rester vigilant face aux séductions du langage.

On trouvera partout chez Gao les marques de cette vigilance vis-à-vis du langage, particulièrement dans la quête du moi. Par exemple, dans le chapitre 58 de La Montagne de l'âme:

Tu rampes dans le langage en traînant tes pensées pesantes. Tu voudrais tirer un fil conducteur pour t’aider à t'en sortir, mais plus tu rampes plus tu es harassé, tu es ligoté par le fil conducteur du langage; tel un ver à soie qui tisse son fil, tu fabriques un filet autour de toi, qui t'enserre dans des ténèbres de plus en plus profondes. La faible lumière au fond de ton cœur est de plus en plus ténue et, tout au bout du filet, ce n'est que le chaos ${ }^{60}$.

De même se manifeste dans Dialoguer-interloquer un double doute: sur le moi et sur le langage. Les deux protagonistes (notamment l'homme) cherchent en vain leur moi dans le langage; le moine montre qu'il n'y a que l'illusion du moi. Suit un dialogue de plus en plus extravagant; la femme et l'homme ne peuvent s'arrêter de parler mais, en même temps, ils nient la validité et l'efficacité du langage — « on ne peut pas le dire »:

La femme (plus bas): On ne peut pas le dire.

L'homme (tout bas): Pourquoi?

La femme (avec affirmation): On ne peut pas le dire.

L'homme: Pourquoi ne peut-on pas le dire?

La femme (lui parlant presque à l'oreille): On ne peut pas le dire!

(L'homme est silencieux. Le moine entre en scène. Bruit d'eau. Le moine avance à grands pas. Il pose un genou sur le sol et s'incline. Les mains jointes, il fait le geste de prendre de l'eau; de son auriculaire, il effleure l'eau; il se lave les oreilles; il se lève pour écouter avec respect. Ses lèvres s'entrouvrent pour dessiner le sourire du Bouddha Amitâbha. Il sort de scène sans bruit)

La femme: Mais inopinément elle a parlé, elle a dit ce qu'on ne peut pas dire; elle le dit encore. Ce que clairement on ne peut pas dire, pourquoi veut-elle le dire encore? Ce qu'il ne fallait pas, ce qu'elle ne pouvait pas dire, elle s'est obstinée à le dire. C'est là son malheur, son infortune, c'est là son erreur ${ }^{61}$.

60. GAO, X. J., La Montagne de l'âme, op. cit., p. 470.

61. GAO, X. J., Dialoguer-interloquer, op. cit., p. 60-61. 
Le dialogue de la femme et de l'homme contraste avec le geste corporel du moine: le moi n'est pas ce dont je parle, il est là où je parle et à la fois je ne le dis pas, comme le moine qui écoute le discours mais en même temps l'évacue de ses oreilles (il se lave les oreilles).

Puis Gao emprunte le mode questions-réponses du gong’an:

L'homme: La raison pour laquelle tu es toi, c'est d'abord que tu es toujours à parler.

La femme: Vent.

L'homme: Tu ne comprends pas ce que tu dis. Tu parles juste pour parler. (Il secoue la tête)

La femme: Vide.

L'homme: Tu ne comprends pas toi-même le sens de tes mots. Tu deviens esclave du langage en vain, tu ne peux t'arrêter de parler. (Il secoue la tête)

La femme: Soldat de plomb.

L'homme: Comme une araignée, tu ne peux t'extraire du filet du langage, (Il secoue la tête) non, tu n'es pas une araignée, et pourtant si, tu es une araignée. (Il secoue la tête)

La femme: Bougie.

L'homme: Tu te prends dans le filet de ton langage, malgré toi. (Il secoue la tête)

La femme: Bodhisattva, remettre, abattement, trompette, bois...

L'homme: Ville d'alcool, pleurs funèbres, vieillard de pierre. (En s'écoutant) Une idole de pierre, à quoi bon la pleurer? Toute la ville est ivre ou l'ivresse abat la ville? Sous l'ivresse, elle pleure son idole? Une personne de pierre insensible a des sentiments? La ville est-elle ivre? La pierre le sait-elle?

[...]

(Le moine ne prête plus attention; il balaie activement. Les deux personnages se tordent de plus en plus en suivant le rythme accéléré du balayage; ils ont l'air d'étranges reptiles ${ }^{62}$ )

Il ne semble pas que Gao ait l'intention d'exhiber cette " méthode dialogique " du bouddhisme chán comme un remède pour se retirer du rapport du moi et du langage. En revanche, le mode d'interruption dans les questions/réponses du gong'an fait ressortir l'aliénation des deux sujets parlants par leur langage, comme le geste corporel du moine contraste avec le dialogue de la femme et de l'homme.

62. Ibid., p. 62-65. 
Finalement, les manières et les mouvements de la femme et de l'homme sont de plus en plus anormaux et étranges. Le langage, ce fil que " tu " utilise dans La Montagne de l'âme, devient une fêlure dans Dialoguer-interloquer:

La femme: Fêlure...

L'homme: Quelle fêlure?

La femme: Une fêlure...

L'homme: Quelle sorte de fêlure?

La femme: Fêlure une...

L'homme: Quelle fêlure une?

La femme: Une fêlure...

L'homme: Une fêlure quoi?

[...]

La femme: Une fêlure...

L'homme: (marmonnant) Une fêlure...

L'homme et la femme (presque en même temps): Une fêlure. ${ }^{63}$

Une fêlure entre le sujet parlant et le langage? Ou bien le propre du langage n'est rien qu'une fêlure qui se projette sur le sujet parlant? Il semble que la femme soit aliénée par le langage et devienne la figue concrétisée de la copule " être ": car si la subjectivité émerge par et dans le langage, la poursuite du moi est inévitablement transformée en enquête de la linguistique. L'héroïne dans $\mathrm{Au}$ bord de la vie exprimait déjà profondément cette anxiété impossible à dominer:

De qui s'agit-il dans ce qui est dit, peut-être de lui, peut-être de toi, peut-être de moi, ou d'ELLE, de l'héroïne? Mais lorsqu'ELLE dit " elle ", ce n'est pas ELLE, ni toi, ni moi, ni vous. Ce " elle", tout comme celle que vous voyez en moi, n'est pas non plus vraiment moi. Et ce moi n'est pas moi, ni toi, ni lui, ni ELLE. Aussi, ne devrait-on pas dire « soi »?

Ou bien est-ce un «soi » en vous, ici, maintenant, qui me regarde ou qui est regardé par ce «soi » dont on ne sait qui il est? Que dire d'autre?

Qu'est ce que ce soi-disant " soi »? Et de tous ces mots, que reste-til $^{64}$ ?

63. Ibid., p. 65-66.

64. GAO, X. J., Théâtre 1, op. cit., p. 85. 
L'inexplicable est là: le sujet est-il interrogé par le langage ou au contraire, le langage par sujet? Peut-on concevoir un langage qui n'est pas cohérent du sujet dont on veut parler?

\section{IMAGE ET LANGAGE: NI SUBJECTIVÉ NI OBJECTIVÉ}

\section{Qu'est-ce que le langage évocateur (唤起的语言)? Le langage évocateur et le xing (兴)}

Lorsqu'il emprunte le mode dialogique du gong'an dans lequel la réponse ne correspond pas du tout à la question et où le langage ne fonctionne pas selon l'articulation logique, Gao tente de montrer une autre façon de prendre de la distance avec ce dont il veut parler.

Gao estime que "la nature du langage ne réside pas dans la description »:

La langue n'est pas un art plastique comme la peinture, elle n'a aucun moyen de dessiner la forme ou la couleur d'une feuille d'arbre. Si l'on se livre à ce genre de description à l'aide du langage, plus elle sera minutieuse, plus il sera difficile de reconnaître la feuille. Le langage ne peut pas résumer l'image, il peut seulement suggérer, attirer et réveiller l'expérience que l'homme possède déjà pour l'amener à se remémorer cette image ${ }^{65}$.

[Ce] n'est pas que le langage ne puisse fournir une image visuelle, mais le mérite du langage est plus d'évoquer les choses que de les décrire ${ }^{66}$.

Mais comment un langage qui ne décrit pas «la chose " peut-il suggérer, attirer et réveiller « la chose »? Qu’est-ce que ce langage qui fonctionne à l'évocation? Il semble correspondre au vrai sujet du sentir qui n'est pas tout à fait en moi: le langage et le vrai sujet reflètent la même façon de s'éprouver et d'éprouver le monde. Si l'expression reste " imprécise » c'est qu'elle doit l'être pour rendre un rapport du monde et du moi dans lequel le premier ne se constitue pas en objet pour la conscience du moi, et le second en sujet face au monde.

"Décrire " implique une écriture qui représente "quelque chose " par l'imitation, ce qui ne convient ni au goût de Gao ni à l'esthétique traditionnelle chinoise sur laquelle il s’appuie. Si le monde

65. GAO, X. J., Le Témoignage de la littérature, op. cit., p. 89.

66. Ibid., p. 93. 
n'est pas l'objet de ma conscience, de mon langage et de mon action, il est avec moi ou même déjà, en quelque sorte, en moi. Je ne cherche pas nécessairement à représenter le monde de manière analogique. Comme le dit Mengzi: « Tous les choses sont procédées en $\mathrm{moi}^{67}$ (万 物皆备于我).”

C'est pourquoi Gao renonce à la description et ne cherche pas à représenter réellement "quelque chose " en dehors du soi. Au contraire, il tente d'évoquer ce qui dans le soi assure l'appartenance au monde: c'est aussi à partir de là que naît la poésie chinoise. Pauline Yu dans The Reading of Imagery in the Chinese Poetic Tradition soutient que toute la poésie occidentale tend à s'identifier à la fonction de l'imitation, qui s'articule logiquement avec la problématique centrale de la relation entre l'être et l'apparence dans un contexte ontologique; et elle indique que cette notion fondamentale (l'imitation) n'a pas la même place dans la poésie chinoise ${ }^{68}$. Rappelons la notion de l'« entre " qui échappe à l'ontologie mais s'enracine profondément dans la poésie et l'esthétique chinoises, « ne se laissant pas focaliser en "chose ${ }^{69}$ ". Si la description conduit à focaliser en "chose", le langage évocateur s'émancipe de la "chose " elle-même (la " chose » au sens d'objet). La dernière nouvelle de Gao, Instantanés, est tissée d'une telle écriture. En voici le commencement:

Il est allongé, seul, dos à la mer, sur une chaise longue installée sur la plage. Le vent est fort. Le ciel est clair, pas trace de nuage, la mer reflète la lumière éblouissante du soleil, son visage reste flou.

Une énorme porte en fer, trempée d'humidité, couverte de traces de rouille; depuis son sommet inaccessible au regard, une eau ruisselle jusqu'en bas. Les deux battants de la lourde porte s'ouvrent lentement; au fur et à mesure que l'interstice s'élargit, les klaxons des voitures de la rue s'y engouffrent. Dans l'ouverture, les gratte-ciel masquent la lumière du soleil. Sirène continue des voitures de police. Dans le couloir obscur qui mène vers le salon, la silhouette d'une femme vêtue d'un manteau. Elle n’a pas allumé la lumière, elle hésite

67. MENGZi, «四书章句集注・孟子 • 尽心上》(Sishuzhangju Jizhu), Beijing, Zhonghua shuju, 2010, p. 350.

68. Voir YU, P., The Reading of Imagery in the Chinese Poetic Tradition, Princeton, Princeton University Press, 1987, notamment le premier chapitre; et aussi JULLIEN, F., La Valeur allusive, op. cit.

69. JULLIEN, F., L'Écart et l'Entre: leçon inaugurale de la chaire sur l'altérité, op. cit., p. 55. 
un peu, pose la main sur la poignée de la porte, l'entrouvre furtivement et sort. La poignée reprend sa place lentement et la porte se referme dans un discret claquement.

Le soleil est doux, invitant au sommeil. Il ferme son livre, se renverse dans la chaise longue, chausse des lunettes noires; deux petits verres ronds cachent ses yeux. Ensuite il prend un chapeau noir aux larges bords et le pose sur son visage, il n'entend plus que la houle ${ }^{70}$.

Tout au long de cette nouvelle, l'auteur ne raconte aucune intrigue, il ne fait que déployer les scènes. Il ne s'agit certes pas d'une exposition au hasard: les deuxième et troisième paragraphes s'insèrent entre le premier et le quatrième paragraphes qui sont deux scènes continues. Il semble que les quatre paragraphes constituent comme une ligne de parabole, le premier et le quatrième se trouvent au point de départ et au point terminal, alors que le deuxième et le troisième forment une courbe. Cependant ceux-ci n'impliquent ni une allégorie (justement, en français, le mot parabole a lui-même le sens d'allégorie), ni une métaphore pour les autres: on ne voit pas vraiment un rapport analogique entre les deux. Les deuxième et troisième paragraphes semblent s'étendre vers une autre dimension du niveau de sens et d'écriture et même produisent une sorte de suspension. Mais pourquoi cette suspension? Quel rapport y a-t-il entre cette suspension et le langage évocateur?

Il convient de remonter en arrière dans la littérature chinoise pour comprendre. Ce langage évocateur est à la fois un héritage et l'outil de la modalité du xing (兴) - celle-ci est l'une des notions les plus fondamentales de la tradition esthétique et littéraire mais aussi la plus contestable.

Depuis longtemps le xing est ambigu: il est souvent associé au bi (comparaison/métaphore) au niveau du fonctionnement. Liu Xie ${ }^{71}$ est le premier critique qui distingue clairement ces deux notions dans Wenxin Diaolong (《文心雕龙 »):

En effet, le bi procède par rapprochement, et le xing par incitation. Quand nous procédons par rapprochement au travers de la nature

70. GAO, X. J., Instantanés, dans Une Canne à pêche pour mon grand-père, op. cit., p. 81-82. 71. Liu Xie (刘妿思 465-521) est l'auteur de Wenxin Diaolong Jinshi (Le Cour de la littérature et la Sculpture des dragons) l'un des livres les plus réputés concernant la théorie littéraire en Chine. 
des choses nous mettons en rapport leurs aspects analogiques de façon à mettre en évidence ce que nous voulons exprimer, et quand notre émotion est suscitée, c'est à partir des [effets subtils] émanant de cette incitation [que nous exprimons ce que nous ressentons en nous-mêmes ${ }^{72}$ ].

La différence principale entre le bi et le xing, est que celui-ci ne se réfère pas nécessairement au principe de l'analogie sur lequel celuilà se fonde. Selon Liu Xie, le xing dépend d'une réaction affective: “ C'est par l'évocation d'émotion, que le corps/style du xing s'établit ${ }^{73}$ (起情故兴体以立).”

Le premier poème Guangju (关雎) du Livre des poèmes (“诗经 ") nous offre un bon exemple de la manière dont le mode du xing se différencie celui du $b i$ :

Guan-guan se répondent les orfraies,

Sur un îlot, dans la rivière.

Une jeune fille, charmante et réservée

Pour ce seigneur quelle bonne compagne elle fait ${ }^{74}$ !

Les deux premiers vers qui se plient entièrement à la modalité du xing montrent une paire d'orfraies se répondant mutuellement par leurs cris; cette paire ne rappelle pas l'image de la relation conjugale chez l'être humain, mais évoque l'image d'une jeune fille chez l'homme vertueux/le seigneur qui la désire. Il n'y pas nécessairement de rapport analogique entre les deux parties de la strophe (malgré les interprétations les plus " classiques » qui cherchent la similarité en les transposant sous l'angle politique et moral pour en chercher le sens ${ }^{75}$ ), mais plutôt une incitation à partir de l'émotion qui ne s'identifie pas forcément à l'empathie.

72. Nous prenons la traduction de François Jullien, dans La Valeur allusive, op. cit., p. 178. 73. LIU, X. 《文心雕龙今释 » (Wenxin Diaolong Jinshi), Beijing, Zhonghua shuju, 2010, p. 325.

74. Traduction de François Jullien, La Valeur allusive, op. cit., p. 176.

75. Par exemple, la première phrase de Mao Shi Xu ( «毛诗序》) déclare que Guangju montre l'exemplarité de la relation conjugale pour tous les couples et que Guangju loue la morale de la princesse à la manière de Feng (风, vent, allusion). Cette interprétation est acceptée par les lettrés confucianistes de la dynastie Han et des époques suivantes et est considérée comme l'interprétation la plus classique pour Le Livre des poèmes. 
Le xing émerge lorsque la recherche du sens cède la place à une poursuite spontanée de l'émotion. La structure poétique qui lui cor-

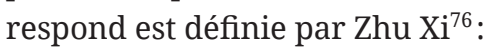

La valeur d'incitation du motif tient à sa capacité de déclenchement, affectif et verbal: l'association qui la caractérise s'analyse sur un plan structurel non plus sémantique (même si celui-ci peut doubler l'autre), le détour qui est en cause est à comprendre comme un phénomène de réaction (émotionnelle) plutôt que de transposition (idéelle). La définition de la figure devient alors: « commencer à parler d'autre chose de façon à introduire à partir de là ce qui est chanté ${ }^{77}$.

La structure - ou bien le rapport " d'antécédent à conséquent ${ }^{78}$ " qui ne renvoie pas forcément à celui de signifiant et de signifié — est une disposition de la chose qui incite et la vraie parole au lieu de « la transposition idéelle ». Autrement dit, cet ordre qui est au-delà de la logique métaphorique consistant à transposer le sens d'une chose à une autre à des fins de dénomination, révèle l'essence du xing. La raison pour laquelle une autre chose/le monde extérieur (il s'agit souvent du paysage) évoque ce qui en moi (ce dont je veux parler) n'est pas seulement l'émotion, mais encore ce qu'on appelle la " subjectivité » du moi qui ne se renferme pas en moi - elle coule vers le monde extérieur en tous sens... Ainsi le rapport du moi et du monde devient réversible.

De ce point de vue, la structure qui est mobilisée par l'incitation — le xing - a la possibilité de se renverser: le paysage m'incite ou je évoque/rencontre le paysage. Xu Fuguan ${ }^{79}$ est d'accord avec Liu Xie en ce qui concerne l'émotion et aussi avec Zhu Xi sur la manière de l'incitation, en examinant les différentes interprétations de cette dernière tout au long de l'histoire de la critique. Il observe encore en suivant les poèmes dans Le Livre des poèmes, qu'il y a différentes structures selon la place où le xing se trouve: au début, au milieu et

76. 朱喜, (1130-1200), lettré et philosophe de la dynastie Song du Sud, il est l'un des plus importants néoconfucianistes.

77. Cité encore par François Jullien, dans Le Détour et l'accès, Paris, Bernard Grasset, 1995, p. 171.

78. Ibid

79. 徐复观 (1904-1982), philosophe néoconfucianiste contemporain. 
à la fin du poème. Xu appelle ce qui apparaît au début du poème, comme ce que nous citons dans Guangju, la modalité originelle du xing ${ }^{80}$. Ce qui apparaît au milieu du poème, Xu le nomme modalité suspension-interruption (顿跌) du xing, modalité dont la poésie ultérieure a hérité (notamment celle de la dynastie Han, par exemple "Les dix-neuf anciens poèmes ${ }^{81}$ (《古诗十九首》). Xu prend un poème du Livre des poèmes pour exemple:

$\begin{array}{ll}\text { Mon seigneur s'enrôle dans le service } & \text { 君子于役 } \\ \text { On ne sait pas sa date de retour } & \text { 不知其期 } \\ \text { Quand reviendra-t-il? } & \text { 曷至哉? } \\ \text { Les poulets perchent dans le trou du mur } & \text { 鸡栖于诗 } \\ \text { Le soleil se couche } & \text { 日之夕矣 } \\ \text { Les moutons et vaches rentrent } & \text { 羊牛下来 } \\ \text { Mon seigneur s'enrôle dans le service } & \text { 君子于役 } \\ \text { Comment ne pas penser à lui ? } & \text { 如之何勿思 } \\ & --- \text { 《王风・君子于役》82 }\end{array}$

D’après Xu, la suspension-interruption (顿跌) qui apparaît entre le troisième et quatrième vers est produite par le sentiment que l'auteur a accumulé - c'est comme une suffocation lorsqu'on parle trop rapidement. Cette suspension-interruption fait sortir l'auteur de ses sentiments et le fait rencontrer les autres « objets " placés en dehors de lui (des poulets, du soleil, des moutons et des vaches). Ceux-ci produisent une sensation encore plus profonde et plus tortueuse sur l'auteur: un Monde émotionnel (qingjing 情境) est ouvert où le sujet du poème est approfondi ${ }^{83}$.

$\mathrm{Xu}$ considère le xing qui se trouve à la fin du poème comme le style le plus développé et le plus raffiné de la poésie lyrique classique, un style fréquemment adopté par les poètes de la dynastie Tang. Il nous en tire encore un exemple dans Le Livre des poèmes ${ }^{84}$ :

Le fruit du poivre

Se multiplie et remplit le boisseau

椒聊之实, 蕃衍盈升。

80. XU, F. G., 《释诗的比兴》, dans 《民主评论 》 (Minzhu Pinglun), 1958, vol. 9, n 15, recueilli dans 《中国文学精神》, 上海世纪出版社, 2006, p. 38-39.

81. Ibid.

82. Ibid., p. 39. Nous traduisons.

83. Ibid., p. 39

84. Ibid., p. 40. Nous traduisons. 
Ce prince de celui-là

N'a pas d'égal en grandeur et en puissance

Oh le poivrier,

Étend loin ses branches
彼其之子， 硕大无朋。 椒聊且, 远条且。 $--($ 唐风・椒聊 $)$

Selon $\mathrm{Xu}$, les deux derniers vers ne sont pas simplement une répétition des deux premiers; au contraire, c'est une métamorphose du xing: "Le sens du poème est déjà bien exprimé par les quatre premiers vers, mais l'émotion n'est pas encore finie - celle-ci se projette sur la chose objective et fait que cette chose est contaminée (沾染) pour que la sensation du poète puisse trouver le moyen de subsister par cette chose objective ${ }^{85}$. "

Ce qui est intéressant ici, c'est que même si Xu, en tant que philosophe et sinologue, utilise les termes " objet/objectif " et " sujet/ subjectif » pour expliquer les poèmes traditionnels chinois, il les range en réalité dans la catégorie chinoise du moi-monde, ce qui fait que ces termes se répondent, se " contaminent » et se remplacent l'un l'autre. Ainsi dit-il: « Le plus haut niveau de l'art et de la littérature, est ce qui ouvre dans les choses concrètes et limitées un Monde sans limite où le sujet et l'objet s'unissent de manière très subtile ${ }^{86}$. " S’exprimant ainsi, Xu partage le point de vue de Wang Fuzhi: le xing fait que l'émotion et le paysage se mêlent et se fondent l'un dans l’autre (情景交融).

\section{La réversibilité entre le moi et le monde et entre le moi intérieur et le moi extérieur}

Retournons vers Gao qui tente depuis toujours d'introduire quelque chose de la poésie traditionnelle dans ses œuvres ${ }^{87}$. Le

85. Ibid.

86. Ibid.

87. Cet effort ne se trouve pas seulement chez Gao, mais aussi chez certains écrivains et poètes de Taiwan. Ils introduisent la poésie traditionnelle dans leurs ouvrages en guise de déconstruction (on peut se référer au travail de Yung Man-han 翁文涃, une chercheure de Taiwan, par exemple à son article « Observation of Postmodern Poetry in Taiwan : Writings of Hsia Yu and the New Generation (台湾后现代诗观察: 夏宇及其后的新一代书 写)». Voir cet article entier sur site web : https ://docs. google. com/file/d/0B7qeU44vc7wOWUydnVpR2RjR2s/edit). Comparée à celle de ces écrivains de Taiwan, l'écriture de Gao est beaucoup moins «postmoderne ». 
langage évocateur, un xing gaoien, se présente partout dans son écriture romanesque, de La Montagne de l'âme au Livre d'un homme seul et jusqu'à sa dernière nouvelle, Instantanés.

Du xing au langage évocateur, il ne s'agit pas seulement d'estomper l'écart entre langage poétique et langage romanesque, mais aussi de répondre à la question: qu'est-ce qu'une écriture moderne? D’un côté, le langage poétique chinois se différencie du langage poétique occidental: à l'inverse de l'épopée qui est essentiellement descriptive (avec un puissant recours à la métaphore par exemple), il est plutôt lyrique et non narratif; d'un autre côté, les romans chinois d'avant le mouvement de la Nouvelle Culture, ne sont jamais séparés de la poésie; la plupart d'entre eux ont une construction "incohérente ». Dans le chapitre 72 de La Montagne de l'âme, Gao imagine un long dialogue entre « il » et un critique anonyme: ce dernier reproche à « il » de n'être qu'« un moderniste qui tente en vain d'imiter l'Occident ». Par la bouche de "il », Gao rétorque qu'il ne s'identifie pas au modernisme occidental qu'on lui attribue, et avoue aussi son doute sur le roman moderne:

Il reste perplexe, il ne comprend pas si dans un roman, le plus important, c'est de raconter une histoire. Ou si c'est la manière de la raconter? Ou sinon, si c'est l'attitude de l'auteur envers la narration? Ou bien, si ce n'est pas l'attitude, si c'est la détermination de l'attitude? Ou bien si ce n'est pas la détermination de l'attitude, si c'est le point de départ de la détermination de l'attitude? Ou bien, si ce n'est pas ce point de départ, si c'est le moi du point de départ? Ou bien, si ce n'est pas la perception du moi, si c'est le processus de la perception? [...] Ou bien si l'important n'est pas dans cette nécessité, est-il dans le langage? Ou bien, si ce n'est pas dans le langage lui-même, est-ce dans la saveur du langage ${ }^{88}$ ?

Les questions que Gao pose ne portent pas seulement sur l'importance du roman, mais aussi sur la manière de viser "l'objet " et de resserrer pas à pas l'expression. Cette manière d'écrire ne conduit pourtant qu'à l'impasse du langage chez Gao. La modalité du xing lui offre sans doute une façon de prendre de la distance avec le langage lui-même et aussi avec ce dont il veut parler. Cependant, cette modalité a clairement évolué chez Gao: développant la

88. GAO, X. J., La Montagne de l'âme, op. cit., p. 602. 
"structure " du xing, il change encore ce qui donne son pouvoir évocateur à la langue. La suspension d'Instantanés, qui emprunte à la modalité du xing, n'est pas dictée par l'émotion: dans les quatre premiers paragraphes que nous avons cités, il n'y a aucune exposition émotionnelle, mais une présentation de la perception " froide " par laquelle la suspension (les deuxième et troisième paragraphes) est appelée. En effet, à part dans sa première nouvelle Étoile dans la nuit froide, il y a rarement chez Gao d'" exhibition » de l'émotion. Il remplace le lyrisme poétique par une sorte d' « anti-lyrisme » ou de «lyrisme froid ${ }^{89}$ (冷抒情) » qui, selon lui, caractérise la poétique moderne dans les romans et le théâtre.

Mais remarquons que dans les catégories chinoises, l'émotion et la perception ne sont jamais loin de l'une de l'autre: elles proviennent d'une même façon d'éprouver le moi et le monde. Car, d'une part, ni l'émotion ni la perception ne sont complètement en moi: elles ne représentent pas une expérience purement subjective; d'autre part, la perception n'est pas plus objective que l'émotion - elles sont toutes deux en dehors du dualisme sujet-objet mais sont placées dans une relation interne-externe/moi-monde faite de réponses et d'extensions mutuelles de ces opposés. La différence entre les deux tient plutôt aux directions respectives de leur puissance d'incitation: l'émotion va de l'intérieur vers l'extérieur, la perception de l'extérieur vers l'intérieur.

Si la modalité du xing révèle le procès poétique d'intériorisation lorsque l'émotion et le paysage se rencontrent et s'ouvrent l'un à l'autre de telle façon qu'un Monde émotionnel (情境) émerge, le langage évocateur de Gao va encore plus loin dans la pénétration de l'immanence, en s'appuyant sur la psychologie (qui compte tant pour les écrivains occidentaux depuis Freud). Le goût de la madeleine, chez Proust, entraînait le narrateur je dans un voyage à travers temps; en revanche, le monde mental tel que Gao l'évoque se déploie sur l'espace synchronique et non pas sur l'axe diachronique. Dans la Recherche, l'enjeu est le temps - ou la " configuration du temps » comme le précise Ricœur —, alors que les romans de Gao

89. GAO, X. J., «京华夜谈 » (Jinghua Yetang), le dialogue entre Gao et Ma Shoupeng, dans 《对一种现代戏剧的追求》(La Poursuite d'un théâtre moderne), op. cit., p. 227. 
se réfèrent à une dimension unique qui contient atemporellement plusieurs espaces où je me trouve. Voici un passage où les bruits d'enfants évoquent le souvenir de « tu » qui synchronise le « tu » en enfance avec les enfants:

En face, des rires d'enfants percent l'ombre profonde de la montagne. Au-delà de la rivière s'étendent des rizières, une aire de battage se détache dans l'obscurité. Les enfants sont peut-être en train d'y jouer à colin-maillard. Une bande sombre la sépare des rizières. Un rire de jeune fille résonne. C'est sûrement elle. Dans la pénombre qui te fait face, ta jeunesse oubliée reprend vie. Un jour, un de ces enfants se souviendra lui aussi de son enfance. Un jour, la voix perçante de ces petits diables deviendra plus rude, plus gutturale, plus grave. Deux pieds nus frappant les dalles de l'aire de battage en laissant des traces humides les feront sortir de l'enfance et leur ouvriront le vaste monde. Et tu entends alors le claquement des pieds nus sur les dalles. Un enfant sur la berge joue au bateau avec le métier à broder de sa grand-mère. Elle l'appelle, il se retourne et prend ses jambes à son cou. Le claquement des pieds nus sur les dalles est cristallin. Et dans une ruelle, tu revois sa silhouette avec sa natte noire comme jais. Dans les ruelles du bourg de Wuyi, le vent d'hiver est glacial [...] les cris des enfants résonnent encore sur l'aire de battage. Ils rient et crient en se poursuivant. Plus d'enfance, face à toi, seule l'ombre noire de la montagne demeure ${ }^{90} \ldots$

La perception du son (les rires, le claquement des pieds nus sur les dalles et les cris) est un axe qui s'étend du monde extérieur au monde intérieur, et derechef vers l'extérieur. En le suivant, le souvenir d'enfance de " tu » s'ouvre vers l'aire de battage où les enfants jouent, leur bruit s'identifie à celui de « tu » dans son souvenir («Un jour [...] deux pieds nus frappant les dalles de l'aire de battage en laissant des traces humides les feront sortir de l'enfance et leur ouvriront le vaste monde »). On peut dire aussi que " tu " partage sa perception entre plusieurs sujets percevants qui se trouvent dans plusieurs espaces de temps différents. La perception qui fonctionne de cette manière dans Instantanés est plus subtile et plus riche mais plus difficile à suivre. Dans les quatre paragraphes que nous avons cités, les deuxième et troisième paragraphes sont en effet la concrétisation du monde intérieur de « il » représenté par une figure féminine, « elle ».

90. GAO, X. J., La Montagne de l'âme, op. cit., p. 148-150. 
De « il » à « elle », il y a une évocation de la perception en contraste: « il » se trouve dans un paysage horizontal (la mer, la chaise longue) et lumineux (la mer reflète la lumière éblouissante du soleil), tandis que, pour " elle ", tout est vertical (la porte en fer, au sommet inaccessible au regard, une eau ruisselle jusqu'en bas) et obscur (elle n'a pas allumé la lumière). L'incitation à la perception ne s'arrête pas seulement dans ces quatre paragraphes mais apparaît permanente à travers toute la nouvelle. Voici les paragraphes suivants:

Les vagues envahissent la grève, mais avant qu'elles n'aient le temps de se retirer, elles sont avalées dans un doux bruit de succion par les grains de sable, abandonnant de place en place des traces d'écume jaunâtres.

Son bras qui pend lui démange. Des fourmis grimpent dessus. Une seule d'abord, puis elles se succèdent à la queue leu leu.

Elle dit que quand elle a fait l'amour avec deux hommes, devant la cheminée, cela l'a particulièrement excitée. Elle est allongée en travers du lit, la tête appuyée sur le rebord, les yeux fermés, hors de la lumière. La lampe n'éclaire que sa chevelure éparse, ses sous-vêtements et son collant jetés sur le sol.

Il a l'impression que la marée monte, les vagues arrivent sous son fauteuil où elles ondulent un peu avant de se retirer. Une mélodie ancienne flotte dans les airs, triste et belle, comme la plainte funèbre d'une paysanne, comme le son plaintif d'un vieil orgue à bouche ${ }^{91}$.

Du corps de "il " à celui de " elle ", il y a une extension et une amplification de la sensation de la vision, de l'ouie, du toucher, de l'extérieur du moi («il ») à l'intérieur du moi (« elle»). Puis, la perception charnelle de " elle " retourne encore vers « il »: «Il a l'impression que la marée monte... » (La marée montante chez Gao est toujours une allusion à la sexualité et particulièrement à l'orgasme). Dans ce processus d'évocation des perceptions de la femme et de l'homme, il n'y a plus de frontière entre la perception « réelle " reçue de l'intérieur ( « il ») et la perception " fantastique », mais présente comme image mentale (" elle»).

Comme dans le xing où l'émotion tend à être impersonnelle, dans le langage évocateur, la perception s'implique dans une sensation neutre et « froide ». Gao tente d'effacer le visage du sujet percevant

91. GAO, X. J., Instantanés, dans Une Canne à pêche pour mon grand-père, op. cit., p. 82. 
à qui le monde mental appartient - le monde mental n'est pas seulement montré par la sensation corporelle féminine, mais aussi par le paysage:

Une chaise vide flotte sur l'eau, toute droite, dans les reflets des vagues. On n'entend aucun bruit. Seul un son très long oscille dans les airs, continu et monotone.

Un petit garçon sanglote en silence au coin d'un mur de pierre couvert de lierre. Le soleil l'éclaire à mi-hauteur.

Sur une pelouse vert émeraude fraichement tondue, un homme d'un certain âge, vêtu d'une chemise blanche au col largement ouvert, le pantalon tenu par des bretelles. Il tire une corde. Il a l'air de faire des efforts, mais ne se presse pas.

Il est debout dans la rue, devant une vitrine. Au début, il n’y prête pas attention, puis il commence à lire très attentivement ce qui est écrit à l'intérieur. La rue est déserte, à part un ou deux passants ${ }^{92}$.

Si le xing fait surgir l'émotion pour parler d'autre chose sans qu'il y ait nécessairement rapport, entre les deux c'est sans doute parce que le moi et le monde sont sur la même « fréquence », celle du rapport moi-monde fondamental. Chez Gao - comme dans la poésie traditionnelle où la poétique/le monde émotionnel se déploient dans une sorte d'interaction mutuelle de l'émotion et du paysage - le langage ne joue pas de l'évocation, si l'on peut dire, seulement dans le rapport moi-monde, mais aussi sur l'axe de la réversibilité du monde intérieur et extérieur. Gao s'attribue de ce point de vue une parfaite liberté de va-et-vient entre la sensation extériorisée et la sensation intériorisée ( « il » » et image mentale " elle »), et aussi entre le moi et le paysage, en suivant le fil de la perception évocatrice.

Cependant, le moi chez Gao a ses deux côtés paradoxaux: le moi qui s'abrite dans le rapport moi-monde lorsqu'il tend vers un je impersonnel; le moi qui s'interroge sur lui-même ou doute de lui-même lors qu'il prend conscience de soi en tant que sujet individuel. Dans beaucoup de ses pièces de théâtre, le moi intérieur ou le monde mental se concrétise en jouant un rôle. Par exemple, l'ombre de l'homme dans L'Autre Rive, les figures féminines dans Au bord de la vie, la femme et l'homme morts dans Dialoguer-interloquer, le parleur B dans Le Quêteur de la mort, ELLE dans Ballade

92. Ibid., p. 84-85. 
nocturne. Tous ces rôles révèlent une anxiété, un doute ou un conflit portant sur le moi et sur le langage qui colle trop au moi pour s'en détacher dans la manière de s'interroger ou de dialoguer. Le rapport du moi et du langage est finalement paradoxal: le moi qui prend conscience de soi ne peut se confirmer que dans et par le langage; le langage qui exprime le moi remplace cependant le moi. Ils sont nés l'un de l'autre, ils sont inséparables par leur façon de s'exprimer et de se "torturer " l'un l'autre. Ainsi, le monde mental n'est plus représenté par la perception ou le paysage, mais par le langage ou bien par les mots. Dans la bouche des protagonistes des pièces Au bord de la vie et L'Autre Rive et Ballade nocturne, cette relation paradoxale s'exprime déjà en toute profondeur. Ballade nocturne est une pièce de théâtre d'un seul acte où il y a trois rôles féminins: LA COMÉDIENNE, LA DANSEUSE MÉLANCOLIQUE et LA DANSEUSE DYNAMIQUE. Les trois ne représentent qu'un seul et même personnage - ELLE - , qui erre la nuit dans la rue " avec son petit esprit » — toute la pièce relève du monologue poétique. LA COMÉDIENNE représente un moi extérieur parlant, ELLE est le moi intérieur parlant, tandis que LA DANSEUSE MÉLANCOLIQUE et LA DANSEUSE DYNAMIQUE sans parole révèlent concrètement la perception et le sentiment d'ELLE. Voici une parole d'ELLE:

\section{ELLE:}

Si je vous ai dit elle,

Ce n'est pas moi qui le disais.

Si elle vous dit que je voulais dire, c'est forcément elle qui voulait vous dire,

Et moi plutôt au nom d'elle, qui vous parlais, est-ce clair?

[...]

Elle aspire les mots l'un après l'autre successivement dans sa matrice, vifs et sages comme les enfants;

elle attend qu'ils soient conçus de son sang.

[...]

les mots sont tout autant fidèles

à leur maître qu’à leur maîtresse.

Mais maîtresse de qui ? ${ }^{93}$

93. GAO, X. J., Ballade nocturne, Paris, The Cahiers Series (The American University of Paris), p. 4-6. 
Mais après une longue quête monologique du moi et des mots, la pièce de théâtre se termine par le retour du personnage dans le monde quotidien:

\section{LA COMÉDIENNE:}

Elle, après s’être abreuvée le café au lait au petit-déjeuner, ne vivra pas plus que d'autres de sa propre nature.

Et bientôt, elle sera assimilée à la houle de la foule, Comme une ombre en plein jour, en songe.

(ELLE se tourne, le dos au public, va vers le fond de la scène, tandis que les deux DANSEUSES s'avancent vers l'avant-scène. Une projection sur l'écran de la scène montre la foule en marche dans un boulevard. Le ronronnement des voitures submerge la musique $e^{94}$.)

Au bord de la vie et L'Autre Rive s'achèvent de la même façon — la vie quotidienne se profile après la poursuite du moi —, dans L'Autre Rive, les acteurs entrent tous sur la scène, jouent en tant qu'acteurs eux-mêmes et font apparaître le monde quotidien:

Des pleurs de bébé.

Mon chéri, oh, j’ai oublié de changer ta couche!

On entend démarrer une moto.

Comment rentres-tu? C'est nul, qu'est-ce que c'est que cette pièce!

Qu'est-ce que tu fais demain? On mange ensemble?

Pleurs de bébé, bruits de voitures qui démarrent et se mettent en marche, sonnettes de bicyclettes ${ }^{95}$.

En effet les bruits de voitures sont comme un signe du monde extérieur. Ils se font entendre aussi dans $\mathrm{Au}$ bord de la vie pour terminer la pièce:

Elle se replie sur elle-même et s'allonge par terre. L'ombre de la pierre bouge imperceptiblement, les bruits sourds de la circulation des voitures deviennent perceptibles. Immobile, la femme s'est étirée dans la pénombre. On ne sait plus s'il s'agit d'une personne ou d'une masse de vêtements ${ }^{96}$.

Il semble que l'apparition du monde extérieur soit une expression du xing/langage évocateur: seulement lorsque la langue ne vise plus à quelque chose et qu'elle est rejetée, le moi pourrait se fondre et vaciller avec le monde, et le monde serait alors réversible, du moi intérieur au moi extérieur.

94. Ibid., p. 16

95. GAO, X. J., Le Quêteur de la mort, suivi de L'Autre Rive et La Neige en août, op. cit., p. 116.

96. GAO, X. J., Théâtre 1, op. cit., p. 85. 


\section{Le yixiang (意象) est un processus}

Dans son dialogue avec son ami Ma Shoupeng au sujet du théâtre, Gao estime que la meilleure façon de faire apparaître la poésie moderne sur la scène - le lyrisme froid - c'est d'utiliser l'image intuitive produite par le langage, pas celle que suggèrerait le lyrisme traditionnel ${ }^{97}$. De même, Gao a commenté dans son article «Le chinois moderne et l'écriture littéraire " la motivation qui l'a poussé à écrire Instantanés: cette nouvelle est "le résultat de mes efforts pour tenter d'éveiller la perception d'images, y compris des images intérieures ${ }^{98}$ ". Qu'est-ce qu'une telle image? Gao utilise le terme yixiang 意象 pour l'« image », prise en tant que notion centrale présente à travers toute la diversité de ses œuvres (romans, drames, peintures et films), et offrant certains caractères liés à la structure de son langage évocateur.

La raison pour laquelle ce langage évocateur est une métamorphose de la modalité du xing ne tient pas seulement à sa vertu d'incitation, mais encore au fait que le langage évocateur et le xing sont tous deux un langage d'" image " - autrement dit, que ce soit à partir de l'émotion ou de la perception, cette évocation fonctionne à l'aide de l'" image ».

Cette « image », c'est donc le yixiang (意象); la traduction littérale en français « sens-image », qui du coup suggère « le sens de l'image » ou "l'image du sens ", ne correspond pas vraiment à la signification du terme. Le yixiang ne s'identifie pas forcément à l'image ou à l'icône représentée par la métaphore: il ne faut pas voir le yixiang sous l'angle sémantique ${ }^{99}$. De même que la valeur d'incitation du xing ne relève en rien d'une "logique " d'analogie, le yixiang

97. GAO, X. J., La Poursuite d'un théâtre moderne ( 《对一种现代戏剧的追求 »), op. cit., p. 227.

98. GAO, X. J., Le Témoignage de la littérature, op. cit., p. 93.

99. D'autant plus que la métaphore, qui implique d'emblée son caractère prédicatif, comme Derrida le montre en citant Heidegger, est « en particulier déterminante pour la manière dont nous nous représentons l'être du langage. C'est pourquoi la métaphore est souvent utilisée comme moyen auxiliaire dans l'interprétation des œuvres poétiques ou, plus généralement, artistiques. Le métaphorique n'existe qu'à l'intérieur des frontières de la métaphysique » ; voir Jacques Derrida, Marges de la philosophie, Paris, Minuit, 1972, p. 269-270, notamm. la note 19 . 
n’apparaît pas en suivant l'articulation du langage - en outre, la langue chinoise n'a pas une grammaire aussi cohérente que la grammaire occidentale; le yixiang ne transmet pas nécessairement l'information comme le fait l'image dans la métaphore. C'est sans doute la raison pour laquelle le yixiang est une notion partagée dans la peinture chinoise, notamment celle du style xieyi (写意) qui ne représente pas « réellement » les figures. Ainsi Wang Tingxiang ${ }^{100}$ écrit:

Ce qui est appréciable dans la poésie, c’est le yixiang qui est cristallin et transparent, et qui ne colle pas à la chose et à la réalité: il est justement comme, on disait jadis, la lune dans l'eau et l'image dans le miroir qui peuvent être vues mais ne peuvent être atteintes ${ }^{101}$ (夫诗 贵意象透芗，不喜事实粘着，古谓水中之月，镜中之影，可 以目睹, 难以实求).

De ce point de vue, le yixiang est à la fois perceptible et non tangible: il est entrevu dans le langage, mais il n’est pas décrit ou narré par le langage.

Mais, si ces caractères du yixiang sont apparus dans la langue de la poésie chinoise classique et lui conviennent, comment le yixiang peut-il se revitaliser dans un langage modernisé et dans une écriture romanesque?

Lorsque Paul Ricœur explore la métaphore dans l'espace de la sémantique, il cherche quand même entre sens et sensation une liaison qui "puisse être accordée avec la théorie sémantique ». Ainsi, l'explication la plus satisfaisante pour l'image de la métaphore, « la seule en tout cas qui puisse s'harmoniser avec la théorie sémantique, est celle que Marcus B. Hester rattache à la notion, d'origine wittgensteinienne, du "voir comme ${ }^{102}$ " ". Car le " voir comme " est "la relation intuitive qui fait tenir ensemble le sens et l'image ${ }^{103}$ ". Ricœur cite la définition de Marcus B. Hester pour montrer comment le « voir comme " fonctionne comme une médiation: " Mais voir

100. 王廷相 (1474-1544), lettré et gouverneur de la dynastie Ming.

101. WANG, T. X., 《王廷相集 - 与郭价夫学士论诗书»Wang Tingxiang Ji, Beingjing, Zhonghua Shuju, 1989, p. 502-503.

102. Ricoeur, P., La Métaphore vive, Paris, Seuil, 1975, p. 268.

103. Ibid., p. 270. 
comme [...] est un acte-expérience de caractère intuitif, par lequel on choisit, dans le flot quasi sensoriel de l'imaginaire que l'on a en lisant la métaphore, les aspects appropriés de cet imaginaire ${ }^{104}$. "

Il semble que Ricœur renonce au concept du voir en tant qu'acte intellectuel, concept dominant depuis Platon dans La République. Ainsi ce « voir comme " contient un " voir » intuitif et un « comme " intellectualisé ou verbal:

"Voir comme ", c'est à la fois une expérience et un acte: d'une part, le flot des images échappe à tout contrôle volontaire: l'image survient, advient, et nulle règle n'apprend à " avoir des images "; on voit ou on ne voit pas [...]. D'autre part, "voir comme" est un acte: comprendre, c'est faire quelque chose; l'image, a-t-on dit plus haut, n'est pas libre mais liée; et en effet le « voir comme » ordonne le flux, règle le déploiement iconique ${ }^{105}$.

Or, ce " voir intuitif " que Ricœur cherche pour la partie sensible de la métaphore est le caractère essentiel de la notion du yixiang. Pour autant que celui-ci introduit une manière de voir, il s'agit d'un « voir » par l'esprit et l'imaginaire, et non pas par l'œil et par l'acte. Le mot yixiang évoque même une "vision " poussée au-delà de l'image pour aller jusqu'à un état fuyant et vague (冥 漠恍惚之境 $\left.{ }^{106}\right)$; il est avant tout rattaché à une vision intérieure qui implique un " voyage de l'esprit ${ }^{107}$ » implicitement séparé de la vision réelle.

Dans cet esprit, lorsque Gao utilise le terme yixiang ${ }^{108}$ (意象), il réfléchit à la façon de montrer l'image mentale dans le langage et de lier l'image intuitive et le langage:

104. Ibid.

105. Ibid., p. 270.

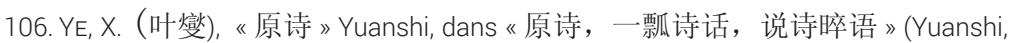
yipiaoshihua, shuoshizuiyu), Beijing, Renmin Wenxue Chubanshe, 1979, p. 39 :«诗之至处, 妙在含蓄无垠，思致微渺，其寄托在可言不可言之间，其指归在可解不可解之会，言 在此而意在彼, 泯端倪而离形象, 绝议论而穷思维, 引人于冥漠恍惚之境所 以为 至也。»

107. Voir par exemple chez Liu Xie, dans «文心雕龙今释》 (Wenxin Diaolong Jinshi), op. cit., le chapitre de Shensi (神思), p. 246-254.

108. La traduction en français de yixiang chez Gao est variable selon le contexte: image, image mentale, imagination... Nous réintroduisons yixiang pour remplacer les traductions et pour éviter les confusions de sens. 
Je ne décris pas directement les images psychologiques, les hallucinations, les souvenirs et l'imaginaire, je recherche soigneusement les sensations psychologiques, et le yixiang ne devient que de simples mots ou expressions dans la phrase. Je me sers de ces yixiang suscités par ces mots et ces expressions comme d'un matériau que je dispose dans le courant de mes phrases. Sans limite fixe, sans utiliser trop d'éléments supplémentaires, à l'aide d'une série de phrases courtes et séparées, sans lien entre elles, je prolonge le processus de réalisation de la langue pour parvenir à une sensation psychologique ${ }^{109}$.

Gao, dans le chapitre 23 de La Montagne de l'âme propose une expérimentation de cette idée:

Tu dis que tu viens de rêver, endormi sur elle. Elle dit que c'est vrai, il y a un instant, elle parlait encore avec toi, tu ne dormais pas, elle dit qu'elle te caressait et pendant que tu rêvais, elle a touché ton pouls, il y a une minute à peine. Tu dis que c'est vrai, tout était encore distinct, tu sentais la douceur de ses seins, la respiration de son ventre. Elle dit qu'elle te serrait, qu'elle a touché ton pouls. Tu dis que tu as vu s'élever la surface noire de la mer, la surface parfaitement plane s'est soulevée lentement, inexorablement. Comprimée, la ligne entre ciel et mer a disparu et la surface noire a occupé tout l'espace. Elle dit que tu as dormi, collé contre sa poitrine. Tu dis que tu as senti ses seins monter, comme une marée noire, que le flux était tel un désir qui enfle, de plus en plus fort; quand elle allait t'engloutir, tu dis que tu as ressenti une sorte d'inquiétude. Elle dit: tu étais sur ma poitrine comme un enfant sage, seul ton pouls s'est accéléré. Tu dis que tu as senti une sorte d'oppression, cette marée qui montait et s'étendait d'une manière incoercible est devenue une immense surface plane qui a déferlé vers toi, sans la moindre vague, lisse et glissante comme une soie noire déployée sans fin, elle s'écoulait, sans rien pour la retenir, puis elle s'est transformée en une chute d'eau noire tombant d'un point invisible situé très haut vers un gouffre insondable, sans rencontrer la moindre aspérité sur son chemin. Elle dit: tu es vraiment stupide, laisse-moi te caresser. Tu dis que tu as vu cet océan noir avec ses vagues déferlantes, cette surface qui s'est soulevée pour occuper tout l'espace, inexorablement. Tu étais sur ma poitrine, dit-elle, c'est moi qui te serrais dans mes bras, avec mon doux parfum, tu savais que c'étaient mes seins, mes seins qui gonflaient. Tu dis que non. Elle dit que si, c'est moi qui t’ai serré, qui ai touché ton pouls qui battait de plus en plus fort. Tu dis que dans ces vagues noires jaillissantes,

109. Le Témoignage de la littérature, op. cit., p. 102. Nous employons yixiang pour remplacer la traduction par « image » et « image mentale». 
nageait une anguille humide et glissante comme l'éclair, mais qu'elle a été engloutie aussitôt par la vague noire. Elle dit qu'elle l'a vue et qu'elle l'a sentie. Ensuite, après le passage de la vague, il n'est resté que la grève sans limite, vaste étendue plane de minuscules grains de sable, et, juste après le retrait de la marée, il n'est resté que des bulles et tu as vu alors des corps humains noirs, agenouillés, rampant, se tortillant, enroulés ensemble, se repoussant puis s'emmêlant de nouveau, affrontés dans un silence absolu sur l'immense grève au bord de la mer, sans bruit de vent, ils s'enchevêtraient les uns aux autres, se dressaient, retombaient, têtes et jambes, bras et pieds inextricablement mêlés. On aurait dit des éléphants de mer, mais pas tout à fait, ils roulaient, se dressaient et tombaient, roulaient à nouveau, se redressaient et retombaient. Elle dit qu'elle l'a ressenti en toi, après de violentes palpitations, ton pouls s'est calmé, puis, par intermittence, les palpitations sont revenues, puis se sont apaisées, elle a tout ressenti $^{110}[\ldots]$

Il semble que ce chapitre emprunte à la construction de la symphonie. La parole de " tu " et celle de " elle " se décomposent en un thème principal et en un thème secondaire: la parole de " elle » contredit la parole de " tu ", la suit ou la réexpose; la parole de « tu " s'élance comme un mouvement dans lequel les images se déploient et se transforment, poussée qu'elle est par son propre rythme linguistique et par le dialogue avec « elle ». Gao a sa propre interprétation pour ce chapitre:

Je fais seulement appel aux impressions qu'ils me laissent, et je les organise dans le courant de la langue, je traite les yixiangs de mes rêves comme des motifs qui apparaissent et se transforment sans cesse, comme une musique, donnant à la langue une sorte de rythme ${ }^{111}$.

En effet, le yixiang pour Gao n'est pas seulement une notion à laquelle il faut réfléchir dans le langage; elle concerne aussi dans la peinture. C'est pourquoi ses écrits et ses peintures s'inspirent les uns des autres selon son idée du yixiang. Par exemple:

Dans le style xieyi, les blancs sont encore plus libres et on ne cherche pas à imiter l'expérience visuelle. Dans la peinture traditionnelle chinoise, le vide est plus un espace psychologique, les grandes surfaces blanches ne servent pas seulement à recevoir les dédicaces,

110. GAO, X. J., La Montagne de l'âme, op. cit., p. 193-194.

111. Ibid., p. 101. 
elles sont aussi nécessaires comme refuge du yixiang et pour faire ressortir le goût de l'encre; remplir totalement la surface du tableau est absolument tabou, car le yixiang ne pourrait plus s'y développer $^{112}$.

Pour Gao, le vide/les surfaces blanches sont la condition préalable pour déployer le yixiang - comme dans la poésie, le yixiang apparaît dans l'" entre » que le langage évocateur provoque. Mais ce vide n'est pas seulement celui de la peinture traditionnelle qui fait se rencontrer le monde extérieur et le monde intérieur; c'est d'abord un « espace " où le monde psychologique du moi se déploie en rencontrant le monde extérieur — puisqu'aussi bien la conscience du moi est désormais incontournable. Ainsi le yixiang chez Gao n'est plus celui de la tradition, qui ignore la " perspective ». Certes, Gao admire dans l'encre chinoise cette fluidité qui parvient à montrer le regard se tournant vers l'intérieur:

Le regard intérieur tellement riche, varié, évolué, changeant, subtil, difficile à capter - évoque une source inépuisable et suggère un grand potentiel pour l'art pictural et le potentiel de l'encre est loin d'être épuisé. Quelle perspective! Ça se pose aussi, de nouveau, la problématique de l'espace. Ce n'est pas l'espace dans le sens de la physique non plus de la métaphysique. Plutôt psychologique mais vraiment visible ${ }^{113}$.

Mais cette perspective ne se réfère ni à l'idée de représenter les objets en mesurant leur position par rapport à l'œil de l'observateur, ni à ce qui est impliqué dans le « voir comme » de Ricœur (pour qui « l'expérience-acte du "voir comme” assure l'imaginaire dans la signification métaphorique: the same imagery which occurs also means $\left.{ }^{114} »\right)$. Faire apparaitre le yixiang ne signifie pas mettre une image sous les yeux et montrer un "spectacle ». Il s’agit cependant d'un processus accessible à tous, qu'il s'agisse de la manière de faire apparaître le yixiang, de la perspective et du yixiang lui-même. Le

112. GAO, X. J., De la création, op. cit., p. 245. Pour la même raison, nous employons directement le mot yixiang au lieu de la traduction « imagination ».

113. GAO, X. J., L'Encre et la Lumière, Paris, Voix/Richard Meier (c'est un livre d'artiste qui n'est pas paginé), 1998.

114. Ricoeur, P., La Métaphore vive, Paris, Seuil, 1975, p. 270. 
chapitre 77 de La Montagne de l'âme déroule tout le processus dans une seule longue phrase:

Le sens de ce reflet n'est pas clair, une surface d'eau réduite, toutes les feuilles des arbres sont tombées, les branches sont gris-noir, l'arbre le plus près ressemble à un saule, un peu plus loin, les deux arbres proches de l'eau sont sans doute des ormes, en face, de fines tiges de saules, ébouriffées, dont les branches dépouillées se terminent par de petites fourches, on ne sait si la surface de l'eau est gelée, par temps froid, une fine couche de glace la recouvre peutêtre, le ciel est nuageux, comme s'il allait pleuvoir, mais il ne pleut pas, rien ne trouble le calme, pas un frémissement à la pointe des branches, pas de vent, tout est figé, comme si tout était mort, seule une petite musique flotte dans l'air, inaccessible, les arbres sont un peu tordus, les deux ormes penchent légèrement, l'un vers la gauche, l'autre vers la droite, le tronc du grand saule s'incline vers la droite trois branches de la même grosseur partent du tronc vers la gauche, assurant un certain équilibre à l'arbre, ensuite, plus rien ne bouge, comme la surface de l'eau, morte, peinture achevée qui n'est plus soumise à aucun changement, la volonté même de changer a disparu, ni trouble, ni élan, ni désir, la terre, l'eau, les arbres, les branches, à la surface de l'eau des traces marron-noir, pas vraiment des îlots, des bancs de sable, des javeaux, de petites parcelles qui affleurent à la surface dont elles rompent la monotonie presque artificielle, au bord poussent quelques arbustes que l'on ne remarque pas, complètement à droite, ils écartent leurs branches, comme des doigts secs, comparaison peut-être peu adaptée, ils écartent leurs branches, c'est tout, ils n'ont pas l'intention de les replier alors que les doigts peuvent se replier, ils sont sans charme, tout près, sous le saule, une pierre, estelle là pour permettre aux gens de s'asseoir et de prendre le frais? $\mathrm{Ou}$ pour permettre aux passants de poser leurs pieds dessus pour éviter de mouiller leurs chaussures quand l'eau est grosse? Aucune de ces raisons n'est peut-être la bonne, peut-être seulement deux mottes de terre, un chemin passe là-bas, ou quelque chose qui s'en rapproche et qui traverse cette surface couverte d'eau ${ }^{115}$ ? Tout est peut-être inondé quand les eaux sont grosses, au niveau de la première branche du saule, on dirait une digue, sans doute la berge quand l'eau est haute, mais cette digue est percée de brèches, l'eau peut encore déborder, sur la digue on n'est pas forcément en sécurité, un oiseau s'envole au loin et se pose sur les fines branches du saule, il est difficile à repérer si l'on n'a pas suivi son vol du regard, on ne le verra que s'il s'envole,

115. Suivant la version chinoise, nous changeons le point final en point d'interrogation. 
il est plein de vie, en observant bien, on en voit plusieurs, ils sautillent à terre, au pied de l'arbre, ils se posent puis repartent, ils sont plus petits que celui qui s'est posé sur l'arbre, moins noirs aussi, peut-être des moineaux, et celui qui est caché dans l'arbre doit être un merle, s'il ne s'est pas encore envolé, tout dépend si l'on arrive à le distinguer, la question n'est pas de savoir s'il y en a un ou non, s'il y est ou pas, mais si on ne le distingue pas, cela revient au même, sur la rive opposée, quelque chose bouge, de ce côté-là, sur les touffes d'herbes jaunâtres, une charrette, poussée par un homme et tirée par un autre, courbé, c'est une à bras avec des roues en caoutchouc, elle peut porter une demi-tonne de chargement, elle se déplace lentement, pas du tout comme les moineaux, on ne s'aperçoit qu'elle bouge qu'après avoir reconnu qu'il s'agit d'une charrette, tout dépend de l'idée qu'on en a, si l'on pense que là-bas ce doit être un chemin, c'est un chemin, un vrai chemin, même s'il est rempli d'eau après les pluies, il n'est pas submergé par les eaux, on peut encore remonter par le regard le long d'une ligne ininterrompue au-dessus des buissons d'herbes jaunâtres et retrouver la charrette, mais elle est déjà loin, elle est entrée dans les branches de saule, on croit d'abord qu'il s'agit d'un nid d'oiseau puis, une fois que le regard a percé les branches, on s'aperçoit que c'est une charrette qui se déplace un peu, elle est très chargée, des briques ou de la terre, les arbres dans le paysage, les oiseaux, la charrette, ont-ils conscience aussi du sens de leur forme? Quelle relation y a-t-il entre ce ciel gris, l'eau et son reflet, les arbres, les oiseaux? Le ciel... gris... une étendue d'eau... les arbres dénudés... pas le moindre vert... des buttes de terre... tout est noir... la charrette... les oiseaux... pousser avec force... ne pas bouger... le déferlement des vagues... les moineaux qui picorent... les rameaux... transparents... faim et soif de la peau... on peut tout... la pluie... la queue d'une poule... des plumes légères... couleur de roses... la nuit sans fin... c'est pas mal... un peu de vent... c'est bien... je te suis reconnaissant... dans la blancheur informe... quelques rubans... roulés... froid... chaud... vent... penche et vacille... spirale... maintenant symphonie... énorme... insecte... sans squelette... dans un gouffre... un bouton... aile noire... ouvrir la nuit... partout c'est... impatient... un feu brillant... des motifs minutieux... des soieries noires... un ver... le noyau de la cellule qui tourne dans le cytoplasme... les yeux nés en premier... il dit que le style... a la capacité de vivre par lui-même... un lobe d'oreille... des traces sans nom... on ne sait quand la neige est tombée, quand elle s'est arrêtée ${ }^{116}$.

116. GAO, X. J., La Montagne de l'âme, op. cit., p. 635-638. 
Ce n'est pas seulement un processus de la vision - qui entre et sort entre le monde extérieur et le monde intérieur et où les images d'arbre, d'eau, d'oiseau, de chemin ou bien de charrette apparaissent et flottent en suivant le regard vers l'extérieur et l'intérieur -, c'est aussi un processus qui délaisse peu à peu la recherche du sens ou de la signification: c'est en ce sens que les images deviennent des yixiangs.

Dans la phrase suivante, la neige recouvre le paysage:

Le ciel sombre, le sol couvert de neige plus brillant encore par contraste avec ce ciel sombre, plus de merles, plus de moineaux, la neige a absorbé toute idée et tout sens ${ }^{117}$.

Car le sens est aussi un processus: il se forme, se transforme et voyage d'un yixiang à l'autre. De ce point de vue, Gao est vraiment proche de la poétique chinoise classique: la sensation motive un langage dont l'intention n'est pas d'exprimer clairement un sens, mais de transmettre l'intuition de cette sensation - yixiang attise la perception, le désir, la pensée qui s'écoulent ainsi dans la fluidité du langage.

Dès lors, on comprendra qu'il y ait rarement de point de fuite dans la "perspective " chez Gao. On peut trouver une référence à la notion de perspective dans ses peintures. Voici deux encres chinoises placées sous le même titre « La perspective » :

117. Ibid., p. 639. 


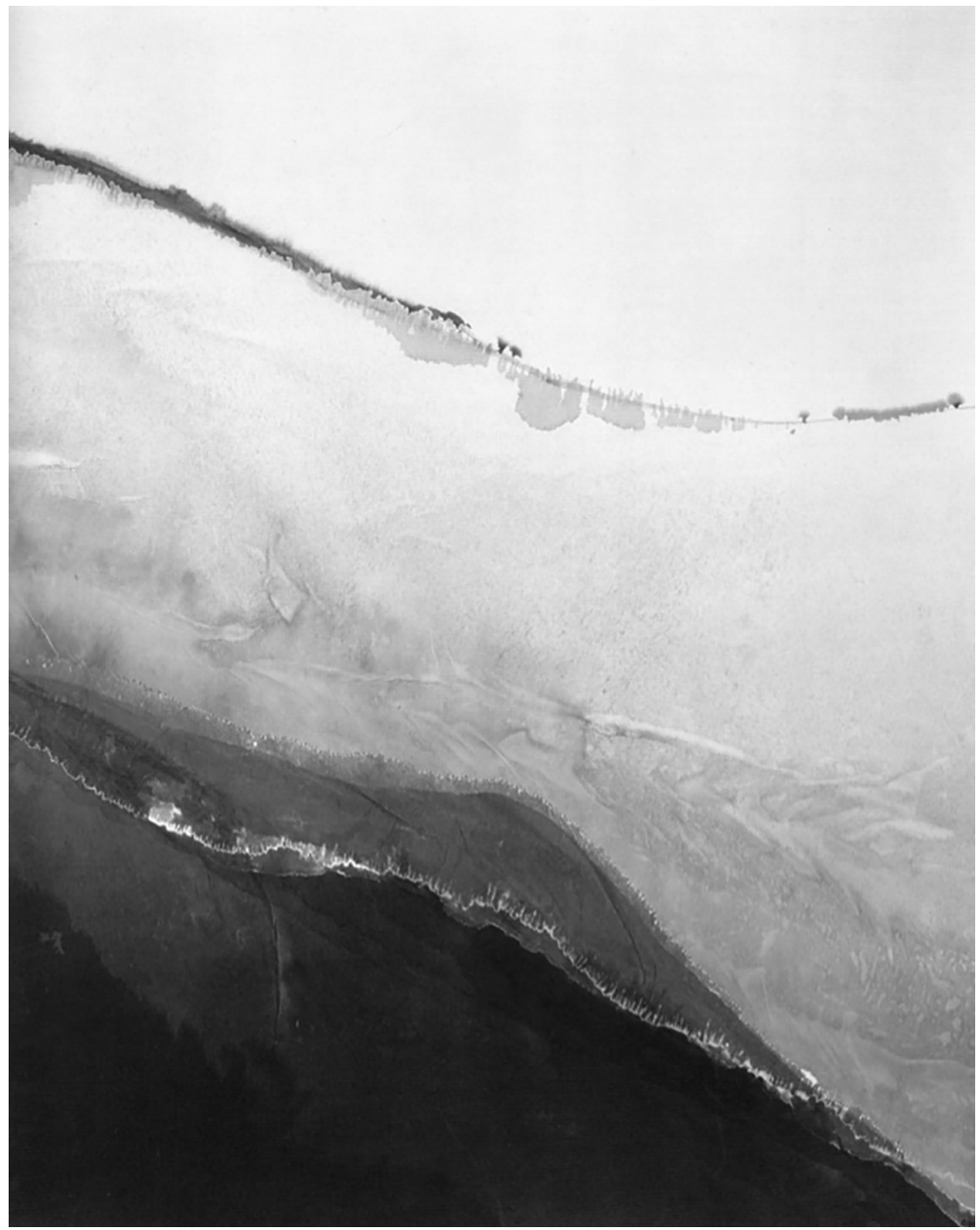

La perspective, 1998 


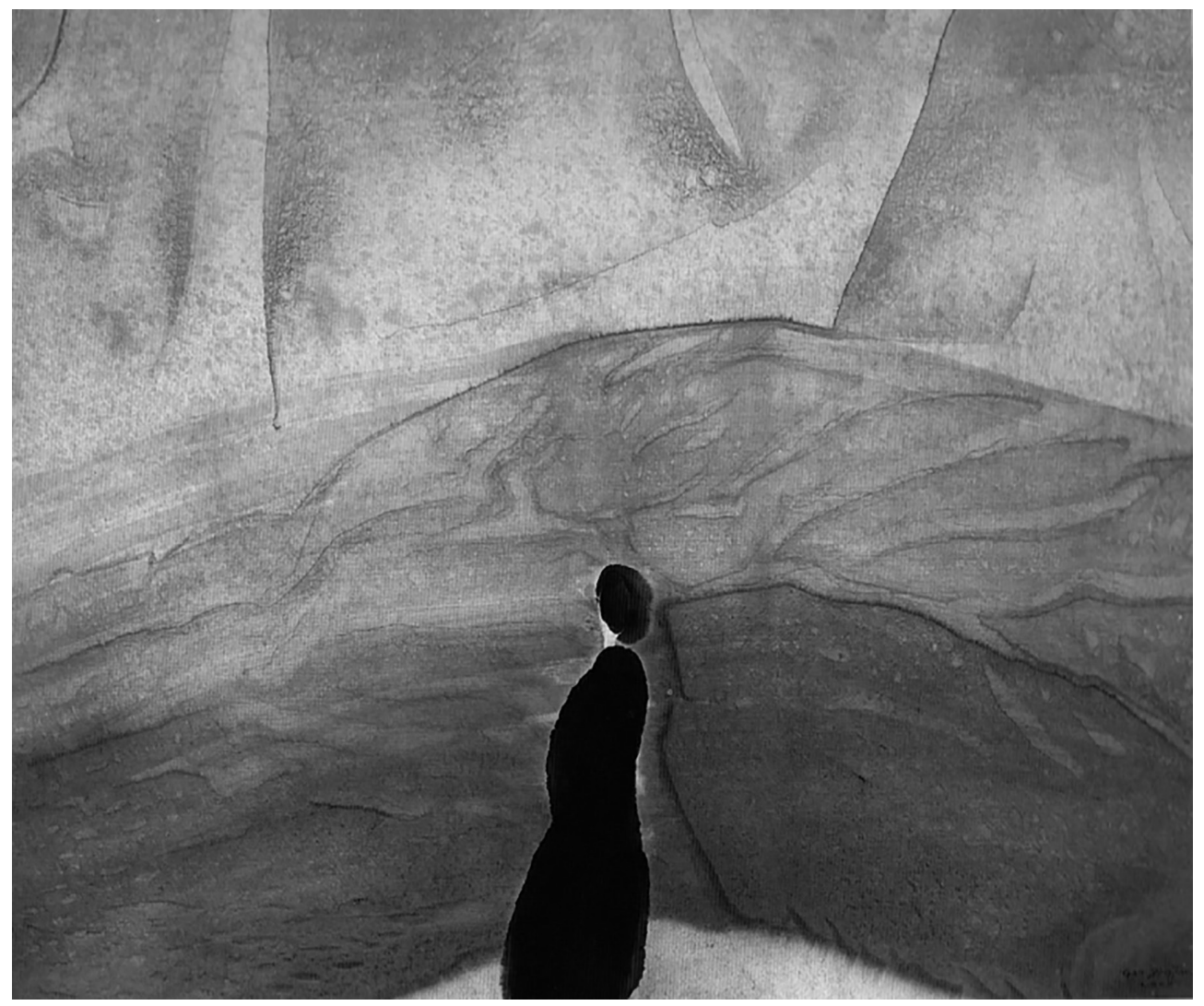

La perspective, 2000

Il n'y a pas de perspective au sens de la peinture occidentale classique ou moderne (par exemple, comme chez les cubistes où la perspective est " déployée » en supprimant la profondeur), et il n’y a même pas une ligne d'horizon; pour mieux dire, il n'y a même pas une fausse ligne d'horizon. Il y a, au contraire, un mouvement ou un processus: la " perspective " que Gao dessine flotte comme un courant d'eau, en changeant l'espace profond en surface plane de plusieurs niveaux de couleurs subtiles entre noir, blanc et gris, et aussi des lignes variées (纹理). La présence ou l'absence du sujet dans le dessin? n’affecte pas la perspective, qui coule et coule... 
Ce retrait de la ligne d'horizon n'est pas simplement lié au fait que, selon Gao, celle-ci n'est qu' « une règle subjective qui dépend de la place où se situe le spectateur ", et qui est définie "à partir d'un point de vue, [alors qu'] elle n'existe pas dans la nature. L'existence ou la non-existence de la ligne d'horizon dépend de l'endroit d'où on regarde ${ }^{118}$ "; plus fondamentalement, pour Gao, il n'y a pas de "vision vraiment objective et définitive ", d'autant plus que "la vision ne peut être fixée ${ }^{119}$ ». C'est que " regarder ne signifie pas que saisir les images du monde réel, mais cela implique aussi, en même temps, une certaine interaction avec l'activité mentale ${ }^{120} »$. Ainsi la perspective fluide fait-elle surgir à la fois la sensation visuelle que l'on éprouve face au monde, pour le monde pourrait-on dire, et l'image mentale prise dans le mouvement de transformation perpétuelle. Ces deux peintures de «la perspective » ne montrent qu'un même yixiang: le processus.

118. GAO, X. J., Pour une autre esthétique, Paris, Flammarion, 2001, p. 30. 


\section{Conclusion}

Le roman n'examine pas la réalité, mais l 'existence. Et l'existence n'est pas ce qui s'est passé, mais l'existence et le champ des possibilités humaines, tout ce que l'homme peut devenir, tout ce dont il est capable.

Milan Kundera, L’Art du roman

Le réel est tellement sensible et tactile; issu de la fusion entre le sujet et l'objet, il est vivant partout et à chaque instant dans la perception de l'homme.

Gao Xingjian, Le Témoignage de la littérature

\section{LA SUBJECTIVITÉ FLUIDE DANS LA TRANSCULTURE}

Nous considérons Gao Xingjian comme un phénomène culturel, plus précisément, un phénomène transculturel: c'est sous cet angle que nous avons voulu entrer dans son monde et déployer la notion de la « subjectivité fluide » dans le contexte de l'évolution du modernisme chinois du $\mathrm{xx}^{\mathrm{e}}$ siècle.

La différence la plus radicale entre les deux cultures ne tient pas au sens ou aux significations de certaines notions et conceptions, mais à la manière de penser et de percevoir qui, en Chine, paraît opérer d'une manière " horizontale ", comme le qi qui souffle entre le ciel et la terre, sans focalisation sur l'« objet " même; tandis que l'approche occidentale est plutôt "verticale ", comme la tour de Babel qui est établie pour toucher le ciel, ériger l'idée en argumentant en visant l'objet lui-même.

Regardons l'étymologie de "culture " en Occident et en Chine respectivement: le terme "culture " est emprunté au latin cultura 
qui signifie l'action de cultiver la terre, puis au sens figuré l'action d'éduquer l'esprit. Tandis que le terme " culture " en chinois, wenhua (文化), composé de deux caractères, la lettre wen et la transformation hua, signifie la " transformation de la lettre ». On voit bien que dès la strate primitive, les modes de penser et de percevoir selon des dimensions respectivement "verticale " et " horizontale " sont déjà marqués. D’autant plus que, dans la transformation de la lettre, le « sujet » et l'" objet » ne sont pas éclairés: qui opère la transformation? et pour qui? C'est la raison pour laquelle la subjectivité-moi est si ambiguë dans la culture chinoise: pas seulement par la façon de la faire émerger et de l'éprouver, mais aussi parce que "qui » est dynamique plutôt que construit. Pour le dire plus précisément, ce "qui-moi » qui ne se réfère pas à un " surplomb » en tant que " moi » unique, est comme l'eau qui coule où la subjectivité se déploie, s'étend et féconde.

Comment la "transculture " ou l' "inter-culture " de Gao vientelle se placer entre deux cultures si différentes? Gao ne cherche pas à bâtir un puzzle culturel qui prendrait des morceaux de plusieurs cultures et les mettrait ensemble, comme le font beaucoup d'artistes et auteurs contemporains; il ne vise pas non plus la «transculture pour elle-même ", comme " l'art pour l'art".

On a demandé à Peter Brook, dans un entretien qu'il a eu avec des étudiantes de l'université Southern Methodist en 1999, pourquoi il pratiquait un théâtre interculturel (notamment entre l'Occident et l’Orient). Brook a répondu:

Quand nous avons commencé à travailler avec des acteurs venant de différents pays du monde, notre propos n'était pas de faire de l'« interculturel ». C'est un slogan, une manière absurde de parler. "Soyons "interculturels", soyons "interraciaux"! » Ça devient anticulturel et même raciste d'employer ainsi de tels termes. Il ne s'agit pas de cela. Il s'agit de quelque chose de beaucoup plus simple: l'être humain n'est pas fini ${ }^{1}$.

L'existence de l'être humain est le point commun essentiel de toutes les cultures: Gao partage le même point de vue que Brook.

1. Brook, P., Entre deux silences, traduit de l'anglais par Marie-Hélène Estienne, Arles, Actes Sud-Papiers, 2006, p. 59 
Car toutes les valeurs et les notions sont douteuses, seule la vie de l'être humain est une vérité ineffaçable. Mais si une transculture se fonde sur l'existence de l'être humain, s'agit-il d'un « humanisme »? Ou bien, s'agit-il d'un regroupement des idées que l'une et l'autre culture peuvent juger " humanistes »? Comment faut-il prendre " humanisme », notion ancrée dans l'histoire culturelle de l'Occident et plus ou moins exportée?

Gao échappe à ces questions qui tendent à transformer la sensation d'existence en idée d'existence ou encore en idéologie. De plus, la transculture ne doit pas pour lui se comprendre en termes de fusion des cultures ni de compromis entre les cultures. Au contraire, Gao fait saillir la particularité du mode de penser et de percevoir chinois en se fondant sur la conscience du moi-individu. Autrement dit, Gao ouvre un espace ou un " écart " entre le mode de percevoir du moi et le moi qui est à la fois le sujet percevant et l'objet perçu. Dans cet écart, le mode de percevoir et le moi ne sont pas opposés l'un à l'autre. Car, d'une part, le moi qui est influencé par l'Occident ne se fonde pas pour autant sur l'être et ne tient un parti-pris du sujet ou de l'objet: ce qui est inspiré de l'Occident est plus la conscience de soi et de l'individu que la façon d'argumenter et de déduire le moi. D'autre part, chez Gao, le mode de percevoir issu de la tradition chinoise lui permet dès le début de ne pas viser l'« objet " perçu. Rappelons que l'« entre " qui constitue la particularité de l'esthétique chinoise n'a rien en propre, ne possède aucun statut: il est inaperçu et invisible. Mais justement, grâce ce "nonpropre ", l'« entre " donne la possibilité de faire passer " tout " par lui - la question d'où remplace celle de qui. Ainsi, chez Gao, le mode de percevoir et le moi sont plutôt parallèles, tandis que la subjectivité qui émerge entre les deux n'est pas constructive et analytique, mais perceptive et dynamique.

\section{UNE SUBJECTIVITÉ NON PRÉDÉTERMINÉE}

Gao met en œuvre un art du roman et du théâtre riche, complexe et fécond qui devance la théorie (même celle de Gao lui-même) et favorise toute une conception de la pensée. À la fois écrivain et théoricien, Gao confirme, à travers ses ouvrages, que ce n'est pas 
la théorie qui dirige l'art, et que ce n'est pas la connaissance qui se place au-dessus du phénomène, car le monde de la perception est, avant tout, un monde qui se transforme sans cesse et qui ne peut jamais être épuisé par la théorie - c'est sur ce postulat que Gao établit son monde littéraire et esthétique.

Gao considère que le siècle dernier fut dominé par l'idéologie, aussi bien en Occident qu'en Chine. L’idéologie ou « le mal du siècle 世纪病 ", dit-il:

Au vingtième siècle, un phénomène très courant est réapparu: la littérature a été contrôlée, maîtrisée, dominée, et même fabriquée et jugée par l'idéologie. Ce phénomène ne concerne pas seulement la création littéraire ou sa critique mais aussi l'histoire littéraire: toutes ont été marquées du sceau de l'idéologie. On peut dire que celle-ci est devenue comme un mal du siècle, une maladie à laquelle il est très difficile de résister. Mais par chance certains écrivains ont su l'affronter, leur œuvre s'en est préservée et continuera à être lue ${ }^{2}$.

Car l'idéologie n'est pas seulement ce qui tend à donner des " explications au monde, des systèmes de valeurs à la société humaine, des fondements cohérents au pouvoir politique et à l'ordre social "; elle est aussi, et avant tout, un mode de penser préalable: "On doit d'abord disposer d'un cadre philosophique et faire ressortir un certain nombre de visions du monde avec leurs valeurs respectives ${ }^{3}$."

Du coup, l'« anti-idéologie " de Gao revêt un double sens. Il s'agit à la fois ne pas s'engager dans la politique et de contrer les idées figées:

Parmi les différentes écoles, le marxisme est sans doute la plus complète quant à la structure et la plus influente quant au rayonnement. Ses idées ont inspiré de nombreuses générations d'intellectuels: en effet, même en dehors des ex-pays communistes, où le marxisme était devenu la structure idéologique officielle, un courant dominant de pensée de gauche balayait déjà l'intelligentsia à travers le monde. Or le libéralisme et le nationalisme sont, eux aussi, tout à fait à même de remplir ce rôle, à savoir devenir des structures de pensée qui favorisent un parti politique ou un pays tout en lui procurant un système de valeurs. Évidemment, l'intelligentsia comprend les mondes litté-

2. GAO, X. J., De la création, op. cit., p. 279.

3. Ibid., p. 280 
raire et artistique, et le modernisme, le postmodernisme et ce que l'on appelle le postcolonialisme peuvent tous devenir des courants de pensée capables de fournir, tour à tour, des jugements de valeur et même des dogmes rigides ${ }^{4}$.

Comment garder l'indépendance du moi face à l'idéologie, une fois que cette même indépendance s'entremêle avec la politique et prend le pouvoir de juger? Lorsque l'individu s'implique dans les idéologies, marxisme ou libéralisme, il risque de se perdre et de s’exprimer d'une façon prédéterminée. On a ou bien l'expression du moi individuel submergé par le nous collectif, comme ce que Gao eu à éprouver en toute souffrance dans son pays natal; ou bien, une expression du moi expansive mais vide, comme l'exprime Alain Badiou:

Comme on dit aujourd'hui: "Je veux être moi-même " — ou, version tribale: " Nous voulons créer, recréer notre propre culture. » Le malheur est que ce vouloir est préformé, et que le "moi-même " ainsi advenu ne se distingue en rien du « tout le monde ${ }^{5}$ ».

La crise du moi est là. Ces deux expressions, celle du nous collectif et celle du moi expansif sont aux antipodes l'une de l'autre, conduisent paradoxalement vers une même crise du moi vide. Raison pour laquelle Gao renonce d'emblée à la recherche de cette identité qui s'attache au pays ou à la nation ou à l'éthique ou à la valeur - le moi indépendant est sa seule identité, inutile de chercher ailleurs; et raison aussi pour laquelle Gao, l'ancien pionnier du modernisme en Chine, en France désigne la modernité comme " une maladie contemporaine ${ }^{6}$ ": celle-là recherche de la " nouveauté » comme le seul principe de l'art ne peut être qu'une " parole creuse » en l'absence d'une réelle qualité personnelle des artistes et écrivains ${ }^{7}$.

Or, la subjectivité fluide que Gao puise dans l'ancienne pensée chinoise est ce qui correspond le mieux à la notion d'individu

\footnotetext{
4. Ibid., p. 279-280.

5. BADIou, A., Circonstances 2, Irak, foulard, Allemagne/France, Clamecy, Léo Scheer, 2008, p. 97.

6. GAO, X. J., De la création, op. cit., p. 177-184.

7. Ibid.
} 
que Gao cherche à bâtir pour échapper à la pensée figée et prédisposée: la subjectivité fluide n'est prédéterminée ni par l’idéologie - marxiste ou libérale -, ni par l'éthique traditionnelle, ni par la substance-entité occidentale.

\section{L'Errance de l'oiseau}

Une subjectivité fluide se déploie horizontalement et ne se construit pas verticalement. Plus précisément, une subjectivité fluide est évidemment horizontale comme l'eau qui coule - c'est dans le courant horizontal du moi qu'elle peut se prolonger et s'étendre. Cependant, cela ne veut pas dire que la subjectivité s'attarde dans un certain " où " et demeure dans un certain «statut »: elle coule dans le moi lorsqu'elle est en moi, ou hors du moi lorsqu'elle prend des distances. Elle est ainsi impersonnelle et sans forme: c'est cette " impersonnalité » et cette " non-forme " qui donnent la liberté/disponibilité à la subjectivité de se transformer sans cesse et, par cette transformation, elle obtient sa vitalité.

Dans son poème L'Errance de l'oiseau ${ }^{8}$, Gao raconte un voyage horizontal du moi: c'est à la fois le voyage spirituel de « tu » et le vol libre de l'oiseau en qui le moi se transforme. En d'autres termes, le moi alterne parallèlement en " tu » et en oiseau, la subjectivité se transforme aussi et se pose entre les deux. Lorsque l'oiseau voltige, tourbillonne à son gré entre le ciel et la terre, " tu " s'envole spirituellement " entre le désert et la mer, à la jonction du jour et de la nuit ». « Tu » est une vision intériorisée qui retourne vers le monde intérieur du moi d'où il fait émerger le paysage — ce « tu » est à la fois le "créateur " et l'observateur de ces images mentales. L'oiseau, lui, est la figure concrète du troisième œil, il provient du moi et aussi de l'observateur hors du moi. Autrement dit, « tu » et l'oiseau sont tous le moi qui est hors du moi ou qui prend de la distance par rapport au moi; ils sont réversibles entre eux.

Mais dans " une luminosité éblouissante " et dans " une transparence du temps » où l'éternel et l'instant s'affrontent, les ombres et la fissure émergent desquelles surgit un "vague oubli ». "Tu »

8. GAO, X. J., L'Errance de l'oiseau, Paris, Seuil, 2003. C'est un livre d'artiste qui n'est pas paginé. 
qui tombe dans l'obscurité - en d'autres termes, qui tombe dans le moi quotidien - comprend qu'il n'est pas un oiseau, et qu'il ne peut plus se détacher du fracas de tous les jours. Puis la mort approchant, " tu " n'a pas encore trouvé un endroit où son âme puisse se réfugier. Pourtant, au moment même où " tu » se retrouve désorienté, il s'identifie de nouveau à l'oiseau et, aussi, au troisième œil: il contemple avec froideur son propre affaiblissement: "Et as-tu jamais vu un oiseau vieux, affaibli, lamentable ou anxieux... ». " Tu » se calme et observe: "[ce lieu saint] que tous les oiseaux savent, après avoir joui de la pleine aisance, le moment venu, ils s'y rendent pour le dernier hommage ".

Le rapport entre cet oiseau d'errance et l'oiseau (peng, 鹏) du premier chapitre xiaoyao you (逍遥游, l'errance à l'aise sans but précis) de Zhuangzi est évident. Mais ce qui est plus remarquable, c'est la manière dont Gao « hérite » du style et aussi de la pensée du xiaoyao you, et les réactualise d'une façon toute subtile dans l'esthétique de la transformation ( $h u a$, 化). Il y a une transformation continuelle entre les moi (dedans ou dehors du moi) en figure de "tu » et de l'oiseau, entre la luminosité et l'obscurité, entre la joie de la vie et l'angoisse de la mort - toutes ces transformations lisses qui ne font pas s'affronter le noir et le blanc sont favorisées par la subjectivité fluide, impersonnelle (sans "parti pris ").

Peut-être L'Errance de l'oiseau est-il le meilleur résumé que Gao ait proposé de sa pensée et de son esthétique: la vie est un voyage de la perception où la subjectivité se transforme de façon ininterrompue; une fois que l'on a un regard hors de soi-même, on se détache de soi-même et s'attribue la liberté/disponibilité comme l'oiseau. 
L'Errance de l'oiseau ${ }^{9}$

Si tu es un oiseau

Rien d'autre qu'un oiseau

Au moment où le vent se lève

Tu t'envoles

Écarquillant ton œil tout rond

Tu regardes dans l’obscurité ce sacré bas monde

Au-delà du marais des ennuis

En vol de nuit, sans but précis

À l'écoute du sifflement de l'air et le cœur battant

Quelle aisance dans l'errance

Tu tournes en un cercle ou vas tout droit, libre de choix Sans obligation de revenir au même endroit

Pourquoi alors reprendre ces minuscules scrupules

Plus de souci, plus de contraintes ni de rancune

Une fois dégagée du fardeau du passé

Ta liberté est au bout des ailes

Tu voltiges, tourbillonnes à ton gré

Un piqué et un rase-mottes ensuite

Et cette terre si lourde autrefois

Commence à balancer avec toi

Tantôt ondule comme une nappe

Tantôt se dresse comme une muraille

La perspective, obsession illusoire jamais aboutie, ainsi disparue

Tant de merveilles successivement inattendues

\section{逍遥如鸟10}

你若是鸟

仅仅是只鸟

迎风而起

率性而飞

眼睁睁俯视

暗中混沌的人世

\section{飞越泥沼 \\ 于烦恼之上 \\ 听风展翼 \\ 这夜行毫无目的 \\ 自在而逍遥}

\author{
盘旋环顾 \\ 或径直如梭 \\ 都随心所欲 \\ 何必再回去收拾 \\ 满地的琐碎 \\ 既无约束 \\ 也无顾虑 \\ 更无怨恨 \\ 往昔的重负 \\ 一旦解除 \\ 自由便无所不在
}

沉沉大地

竟跟随你摇曳

时而起伏

时而竖立

那地平线

本遥不可及

顿时消失了

一个个奇景

全出乎意料

9. GAO, X. J., L'Errance de l'oiseau, op., cit. C'est un livre d'artiste qui n'est pas paginé.

10. GAO, X. J., «游神与玄思》, 台北, 联经, 2012, p. 23-32. 
Brouillard ou nuage

Tu traverses d'un large trait

Et recueilles la lueur et l'aurore

Tout en survolant les montagnes mouvantes

Puis un lac tournant miraculeusement

C'est ainsi que ton esprit circule

Entre le désert et la mer, à la jonction du jour et de la nuit

Tandis qu'un œil immense te conduit vers l'inconnu

Et tu es un oiseau

D'avoir eu une fois un tel regard

Tu t’immerges aussitôt dans la méditation

Révélée par une métaphysique de la vision

Effacée de tout trouble creusé par les mots

Si épurée et limpide que même la distance du proche au lointain s'est fondue dans l'infini

Ce qui est flou, difficile à percevoir

Ce que tu as envie de voir sans oser l'imaginer

D’un coup, tu y accéderas

Dans une luminosité éblouissante,

Le vide, autant que la plénitude

Fait s'affronter l'éternel à l'instant

Une transparence du temps

De certaines ombres ou fissure

Surgit un vague oubli
云或是雾

一掠而过

微光和晨曦

尽收眼底

群山移动

一个湖泊在旋转

犹如思绪

你悠游在

海与旷漠之间

昼与夜交汇之处

偌大一只慧眼

引导你前去

未知之境

凭这目光

你便如鸟

从冥想中升腾

消解词语的困顿

想象都难以抵达

那模糊依稀之处

雬时间在眼前

——浮现

玄思的意境

无远无近

也没有止尽

清晰而光明

明晃晃一片光亮

空如同满

令永恒与瞬间交融

时光透明

而若干阴影与裂痕

从中涌出某种遗忘 
Tout cela que tu n’as jamais eu, aussi éphémère qu'imprévu

Le moindre égarement te fait immédiatement perdre la vue

Et tu bascules de nouveau dans la pénombre Sachant bien que tu n'es pas oiseau Ne parviens plus à te dégager de l'angoisse Qui te harcèle constamment de toute part Et le fracas de tous les jours ne t’épargne pas

Une lutte inutile

Tu devrais au moins trouver un havre Pour le calme de ton âme, si tu en as encore une Un espace quelque part

Un univers, ni paradis ni enfer

Où se pose l'apesanteur de tes sens

Voilà le lieu préservé du dernier jugement Et épargné de la nouvelle utopie L’endroit que les oiseaux réservent au sacrifice Avant que la nature ne les récupère Pour qu'ils se meurent dans le silence Pourtant comment trouver cette terre immaculée Où peut-on obtenir une telle sérénité À la fin de la vie fatiguée et abîmée
这一切从未见过

瞬息变化

难以预料

稍许恍惚

便丧失了

你重又暗中徘徊

清楚的只是

你毕竟不是鸟

也解不脱

这无所不在

总纠缠不息

日常的纷扰

一番徒劳

得找个避风港

安抚你的灵魂

倘若这魂魄

你或许还有

可不该有个去处

既非天堂

也非地狱

令这失重

得以承受

如何找到一片净土

让这困扰的人生

耗尽之际

解除妄念

好得以安息 
Et as-tu jamais vu un oiseau vieux

Affaibli, lamentable ou anxieux

Qui se plaint, qui pleurniche

Qui trompe, qui triche

Sans parler de celui qui quémande la survie

Même si à l'agonie, lui, a déjà préparé son refuge

Là il attend calmement que sa vie s'en aille

Ce lieu saint que tu ne trouves nulle part

Que tous les oiseaux savent

Après avoir joui de la pleine aisance

Le moment venu, ils s'y rendent pour le dernier

hommage
你可曾见过

一只老鸟

衰弱不堪

惶恐不安

凄凄惨惨

哀怨

哭泣

乞求

苟延残喘?

是鸟都知道

悠游了一生

时间来临

便径自奉上

作为祭品

是岛都找好

隐若之地

垂危之际

静静等候

生命消逝

这圣地莫不

也是你的归宿

又在何处? 



\section{Bibliographie}

\section{Ouvrages DE GaO XINGJIAN}

\section{En chinois}

\section{a) Essais et entretiens}

-〈巴金在巴黎〉 《Bajin zai Bali 》, dans 《当代》Dangdai, 1979, n² 2.

- 〈巴黎观剧随笔〉 《Bali guanju suibi 》, dans 《十月》Shiyue, 1979, n 3 .

- 〈关于巴金的传说〉 《Bajin de chuangshuo», dans 《花城》Huacheng, 1979, $n^{\circ} 6$.

-〈巴黎印象記〉 《Bali yinxiangji 》, dans 《人民文学》Renmin wenxue, 1980, n² 2.

-〈法国当代文学的一个主题 一 追求：评两篇法国短篇小说〉 《Faguo dangdaiwenxue de yige zhuti», dans 《十月》Shiyue, 1980, n 3 .

-〈法国现代派人民诗人普列维尔和他的《歌词集》〉 《Faguo xiandaipai renmin shiren Prévert he tade» 《Shiciji》, dans 《花城》Huacheng, 1980, n 5 .

-〈法兰西现代文学的痛苦〉 《Falanxi xiandaiwenxue de tongku », dans 《外国文 学研究》Waiguowenxue yanjiu, 1980, n 1 .

- 〈时代的号手一在巴黎召开的抗战时期中国文学国际讨论会上的发 言〉 《Shidai de haoshou - zai Bali zhaokai de kangzhanshiqi zhongguo wenxue guoji taolunhui shang de fayan », dans 《诗探索》Shitansuo, 1980, n 1.

- 〈文学创作杂记〉 《Wenxue chuangzuo zaji 》, dans 《随笔》Suibi, 1980, Vol.10.

- 《现代小说技巧初探》Xiandai xiaoshuo jiaqiao chutan (Premier essai sur la technique du roman moderne), 广州 Guangzhou, 花城出版社 Huacheng chubanshe, 1981.

- 〈同一位观众谈戏 - 现代戏剧杂谈之一〉 《Tongyiweiguanzhong tanxi xiandaixiju zatan zhiyi 》, dans 《随笔》Suibi, 1982, n6.

- 〈一篇可以当诗读的小說 一 评《和弦》〉 《Yipian keyi dangshidu de xiaoshuo - ping Hexian», dans 《丑小鸭》Chouxiaoya, 1982, n 9. 


\section{Une subjectivité fluide}

- 〈质朴与纯净〉 《Zhipu yu chunjin », dans 《文学报》Wenxuebao, 19 mai, 1983.

- 〈记西柏林〉 《 Ji xibolin 》, dans 《随笔》Suibi, 1986, n 5 .

-〈谈戏曲不要改革与要改革〉 《Tan xiqu buyao gaige yu yao gaige», dans 《光 明日报》Guangming ribao, du 26 Juin 1986.

- 〈用自己感知世界的方式来创作〉 《Yong ziji ganzhi shijie de fangshi lai chuangzuo », dans 《新剧本》Xin juben, 1986, nº 3.

- 〈作家四人谈〉Zuojia siren tan ("La discussion entre quatre écrivains »), dans 《文学自由谈»Wenxue Ziyoutan, TianJin, 1986, nº 5.

- 《对一种现代戏剧的追求》Dui yizhong xiandai xiju de zhuiqiu (La poursuite d'un théâtre moderne), 北京 Beijing, 中国戏剧出版社 Zhongguo xiju chubanshe, 1988.

- 〈戏剧创作需要宽松条件〉 《Xiju chuangzuo xuyao kuangsong tiaojian», dans《明报月刊》Mingbao yuekan, 1992, n 324 (en entier), Vol.27, n 12.

- 〈国家神话与个人癫狂〉 《Guojia shenhua yu geren diankuang 》, dans《明报月 刊》Mingbao yuekan, 1993, no332 (en entier), Vol.28, nº 8.

-〈谈我的画〉 《Tan wo de hua », dans 《明报月刊》Mingbao yuekan, 1993, $n^{\circ} 335$ (en entier), Vol.28, n 11.

- 〈无声的交响 - 评赵无极的画〉 《Wusheng de jiaoxiang - ping Zhao wuji de hua », dans 《二十一世纪》Ershiyi shiji, 1993, nº 19.

-〈中国戏剧在西方：理论与实践〉 《Zhongguo xiju zai xifang», dans 《二十一 世纪》Ershiyi shiji, 1994, nº 20.

-〈戏剧: 揉合西方与中国的尝试〉 《Xiju: rouhe xifang yu zhongguo de changshi », dans 《二十一世纪》Ershiyi shiji, 1994, n² 21.

- 〈当代西方艺术往何处去？〉 《Dangdai xifangyishu wang hechu qu? », dans 《二十一世纪》Ershiyi shiji, 1994, nº 22.

- 〈为什么写作〉 《Weishenme xiezuo》, dans 《沟通: 面对世界的中国文学》 Goutong: miandui shijie de zhongguo wenxue, Stockholm, The Olof Palme International Centre, 1997.

- 〈观照〉 《Guanzhao», dans 《明报月刊》Mingbao yuekan, 1998, n 387 (en entier), Vol.33, $n^{\circ} 3$.

-〈大题小做1〉 《Dati xiaozuo1》, dans 《明报月刊》Mingbao yuekan, 1998, $n^{\circ} 391$ (en entier), Vol.33, $n^{\circ} 7$.

- 〈大题小做2〉 《Dati xiaozuo2 », dans 《明报月刊》Mingbao yuekan, 1998, $n^{\circ} 391$ (en entier), Vol.33, nº 9.

- 〈我把文学创作作为自救的方式〉 《Wo ba wenxue chuangzuo zuowei zijiu de fangshi », dans 《多维周刊》Duowei zhoukan, 13 octobre 2000.

- 〈文学与写作答问- 与金丝燕, 王以培的访谈〉 《Wenxue yu xiezuo dawen » , dans 《二十一世纪》Ershiyi shiji, 2000, nº 62. 
- 〈我与宗教的因缘〉 《Wo yu zongjiao de yinyuan», dans 《亚洲周刊》Yazhou zhoukan, 2000, Vol.14, nº 51.

- 《没有主义》Meiyou zhuyi (Ne pas avoir de -isme), 台北 Taibei, 联经 Lianjing, 2001.

- 《高行健 — 台湾文化之旅》Gao Xingjian - Taiwan wenhua zhilü (Gao Xingjian - un voyage de la culture à Taiwan), 台北 Taibei, 文建會出版社 Wenjianhui chubanshe, 2001.

- 〈关于《侧影或影子 》〉 《Guanyu ceying huo yingzi 》, dans 《联合文学》 Lianhewenxue, 2008, n² 283.

- 〈对诺贝尔文学奖获得者的专访：高行健〉 《Dui Nobei'er Wenxuejiang Huodezhe de zhuangfang: Gao Xingjian», dans 《南方都市报» Nanfang dushibao, mai 2008. http://houhongbinvip.blog.163.com/blog/static/1316567 5620091024430815/

-〈有限与无限 - 创作美学〉 《Youxian yu wuxian - chuangzuo meixue », dans 《香港戏剧学刊》Xianggang xiju xuekan (Hong Kong Drama Review), 2009, nº 8

- 《论创作》Lun chuangzuo (De la création), 香港 Hong Kong, 新加坡青年书局\&香 港明报月刊出版社联合出版 Xinjiapo qingnianshuju \& Xianggang mingbaoyuekan, 2008.

- 《论戏剧》Lun xiju (Du théâtre), 台北 Taibei, 联经 Lianjing, 2010.

\section{b) Nouvelles et romans}

-〈梦海〉 《Menghai », dans 《花城》Huacheng, 1980, nº 6.

- 《有只鸽子叫红唇儿》Youzhi gezi jiao hongchun'er (收录《有只鸽子叫红唇 儿》和《寒夜的星辰》), 北京 Beijing, 十月文艺出版社 Shiyue wenyi chubanshe, 1984.

- 《一个人的圣经》Yige'ren de shengjing, 台北 Taibei, 联经 Lianjing, 1999.

- 《灵山》Lingshan, 香港 Hong Kong, 天地图书 Tiandi tushu, 2000.

- 《高行健短篇小说集》Gao Xingjian duanpian xiaoshuoji, 台北 Taibei, 联合文学出 版社 Lianhewenxue chubanshe, 2008.

\section{c) Pièces de théâtre et opéra}

- 《高行健戏剧集》Gao Xingjian xijuji (收录《车站》, 《绝对信号》, 《野 人》, 《模仿者》, 《躲雨》, 《行路难》, 《喀巴拉山口》, 《独白》8 个戏), 北京 Beijing, 中国戏剧出版社 Zhongguoxiju chubanshe, 1985.

- 《高行健戏剧集1 - 车站》Gao Xingjian xijuji7 Chezhan, 台北 Taibei, 联合文学 出版社 Lianhewenxue chubanshe, 2001.

- 《高行健戏剧集2 - 绝对信号》Gao Xingjian xijuji2 Juedui xinhao, 台北 Taibei, 联合文学出版社 Lianhewenxue chubanshe, 2001.

- 《高行健戏剧集3 - 野人》Gao Xingjian xijuji 3 Yeren, 台北 Taibei, 联合文学出版 社 Lianhewenxue chubanshe, 2001. 
- 《高行健戏剧集4 - 彼岸》Gao Xingjian xijuji4 Bi'an, 台北 Taibei, 联合文学出版社 Lianhewenxue chubanshe, 2001.

- 《高行健戏剧集5 - 冥城》Gao Xingjian xijuji5 Mingcheng, 台北 Taibei, 联合文学 出版社 Lianhewenxue chubanshe, 2001.

- 《高行健戏剧集6 - 山海经传》Gao Xingjian xijuji6 Shanhaijing zhuang, 台北 Taibei,联合文学出版社 Lianhewenxue chubanshe, 2001.

- 《高行健戏剧集7 - 逃亡》Gao Xingjian xijuji7 Taowang, 台北 Taibei, 联合文学出 版社 Lianhewenxue chubanshe, 2001.

- 《高行健戏剧集8 - 生死界》Gao Xingjian xijuji8 Shensijie,台北 Taibei, 联合文学 出版社 Lianhewenxue chubanshe, 2001.

- 《高行健戏剧集9 - 对话与反诘》Gao Xingjian xijujig Duihua yu fanjie, 台北 Taibei, 联合文学出版社 Lianhewenxue chubanshe, 2001.

- 《高行健戏剧集10 - 夜游神》Gao Xingjian xijuji10 Yeyoushen,台北 Taibei, 联合 文学出版社 Lianhewenxue chubanshe, 2001.

- 《八月雪》Bayue xue, 台北 Taibei, 联经 Lianjing, 2000.

- 《周末四重奏》Zhoumo sichongzou, 台北 Taibei, 联经 Lianjing, 2001.

- 《吒问死亡》Kouwen siwang, 台北 Taibei, 联经 Lianjing, 2004.

- 《夜间行歌》Yejian xingge, dans 《联合文学》Lianhe wenxue, 2010.

\section{d) Poèmes}

- 《游神与玄思》Youshen yu xuansi, 台北 Taibei, 联经 Lianjing, 2012.

\section{En Français et en anglais (y compris les traductions du chinois)}

\section{a) Essais et entretiens}

- «Clés pour mon théâtre » (traduit du chinois par Annie Curien), dans Littératures d'Extrême-Orient au Xx $x^{e}$ siècle: Essais, Arles, Philippe Picquier, 1993.

- «Entretien avec Gao Xingjian », dans Sapriphage, juillet 1993, n 18.

- «Entretien », dans Poésie 96, octobre 1996, nº 64.

- «La Voix puissante d'un écrivain en exil », dans Perspectives chinoises, septembre-octobre 1997, n 49.

- L'Encre et la Lumière, Paris, Voix/Richard Meier, 1998.

- «L'Esprit de liberté, ma France », dans Le Monde, au 20 août 1998.

- «Le Peintre de l'âme: entretien avec Robert Calvet », dans Brèves, hiver 1998$1999, n^{\circ} 56$ 


\section{Bibliographie}

- «La Mémoire de l'exil », dans Pourquoi se souvenir?, dirigé par Françoise BarretDucrocq, Paris, Grasset, 1999.

- La Raison d'être de la littérature, suivi d'Au plus près du réel, Dialogues avec Denis Bourgeois, Paris, l'Aube, 2000.

- «La Rencontre: Gao Xingjian », dans L'Aventure du bilinguisme, Paris, L'Harmattan, 2000.

- Pour une autre esthétique (traduit et adapté du chinois par Gao Xingjian, Noël et Liliane Dutrait), Paris, Flammarion, 2001.

- «Gao Xingjian: entretien avec Annie Curien », dans La Nouvelle revue française, janvier 2001, n 556 .

- «Les Difficultés du théâtre chinois contemporain» (traduit du chinois par Myriam Kryger), dans La Nouvelle revue française, 2001, $n^{\circ} 559$.

- «La Voix de l'écrivain » (traduit du chinois par Marie Laureillard), dans La Nouvelle revue française, 2001, n 559.

- «Littérature et écriture, Gao Xingjian : entretien avec Jin Siyan et Wang Yipei » (traduit du chinois par Yang Jianggang), dans Dialogue transculturel, Nice, Alliage, $2001, n^{\circ} 2$.

- "Conversations with Gao Xingjian: The First "Chinese" Winner of Nobel Prize for Literature », dans The China Quarterly, septembre 2001, n 167.

- "Mieux vaut revenir au réel de l'homme », dans Magazine littéraire (Numéro spécial de La Chine de Confucius à Gao Xingjian), mars 2004, nº 429.

- Le Témoignage de la littérature (traduit du chinois par Noël et Liliane Dutrait), Paris, Seuil, 2004.

- Visite à Gao Xingjian et Yang Lian (traduit du chinois par Chantal Chen-Andro), Paris, Caractère, 2004.

- Cold Literature: Selected Works by Gao Xingjian (traduit du chinois par Gilbert C.F. Fong et Mabel Lee), Hong Kong, The Chinese University Press, 2005.

- «La Neige en août en répétition à Taïwan : interview de Gao Xingjian » (traduit du chinois par Marcel Quillévéré), dans La Neige en août, Arles, Actes Sud/Opéra de Marseille, 2005.

- The Case for Literature (traduit du chinois par Mabel Lee), Sydney, Harper-Collins, 2006.

- «Le Troisième œil, entretien avec Gao Xingjian », dans Europe, Jan.-Fév. 2007, $n^{\circ}$ 933-934.

- «Réflexions sur la peinture » (traduit du chinois par Noël Dutrait), dans La Fin du monde, Allemagne, Bielefeld, Kerber Verlag, 2007.

- «Limitée et illimitée, l'esthétique de la création » (traduit du chinois par Noël et Liliane Dutrait), dans Perspectives chinoises, 2010, n 111. 


\section{Une subjectivité fluide}

- Gao Xingjian, Aesthetics and Creation (traduit du chinois par Mabel Lee), Amherst New York, Combria Press, 2012.

- «The Art of Fiction» (traduit du chinois par Mabel Lee), dans Chinese Writers on Writing, Texas, San Antonio, Trinity University Press, collection 'The Writer's World', 2010.

- De la création (traduit du chinois par Denis Molcanov, Noël Dutrait, Stbastian Veg, Yinde Zhang, sous la direction de Noël Dutrait), Paris, Seuil, 2013.

\section{b) Nouvelles et romans}

- La Montagne de l'âme (traduit du chinois par Noël et Liliane Dutrait), Paris, l'Aube, 1995.

- Soul Mountain (traduit du chinois par Mabel Lee), Australia, Flamingo, 2000.

- Une Canne à pêche pour mon grand-père (traduit du chinois par Noël et Liliane Dutrait) Paris, l'Aube, 1997.

- Le Livre d'un homme seul, (traduit du chinois par Noël et Liliane Dutrait), Paris, l'Aube, 2000.

- One Man's Bible (traduit du chinois par Mabel Lee), New York, Harper Collins, 2002.

\section{c) Pièces de théâtre et opéra}

- Wild Man: A Contemporary Chinese Spoken Drama (traduit du chinois par Bruno Roubicek), dans Asian Theater Journal, Honolulu, University of Hawaii Press, automne 1990, Vol.7, n² 2

- L'Arrêt d'autobus (extrait, traduit du chinois par Danièle Turc-Crisa), dans La Remontée vers le jour: nouvelles de Chine (1978-1988), Aix-en-Provence, Alinea, 1988.

- Bus Stop (traduit du chinois par Kimberly Besio), dans Theater \& Society: An Anthology of Contemporary Chinese Drama, dirigé par Haiping Yan, New York, M.E. Sharpe, collection 'Asia and the Pacific', 1998.

- Gao Xingjian Théâtre 1: La Fuite (traduit du chinois), Au bord de la vie, Le Somnambule, Quatre quatuors pour un week-end, Belgique, Lansman, 2000.

- The Other Shore (traduit du chinois par Gilbert C. F. Fong), Hong Kong, The Chinese University press, 2000.

- Dialoguer-interloquer (traduit du chinois par Annie Curien), Belgique, Lansman, 2001.

- Le Quêteur de la mort, suivi de L'Autre rive et La Neige en août (L'Autre rive et La Neige en août sont traduites du chinois par Noël et Liliane Dutrait), Paris, Seuil, 2004

- Ballade nocturne, Paris, The Cahiers Series (the American University of Paris), 2010. 


\section{d) Poèmes}

- L'Errance de l'oiseau, Paris, Seuil, 2003.

- Le Deuil de la beauté, Luxembourg, Simoncini, 2012.

\section{e) Peintures}

- Gao Xingjian, pour une autre esthétique, Paris, Flammarion, 2001.

- L'Errance de l'oiseau, Paris, Seuil, 2003.

- Gao Xingjian : encres, Paris, Galerie Claude Bernard, 2004.

- Gao Xingjian : encres, Paris, Galerie Claude Bernard, 2006.

- La Fin du monde, Allemagne, Bielefeld, Kerber Verlag, 2007.

- Gao Xingjian, Paris, Galerie Claude Bernard, 2008.

- Gao Xingjian Experience, Singapore, Art museum, 2008.

- Gao Xingjian, depuiés del diluvio, Barcelona, Museo Würth La Rioja, 2008.

- Gao Xingjian, Paris, Galerie Claude Bernard, 2011.

- Gao Xingjian: nouvelles œuvres, Paris, Galerie Claude Bernard, 2014.

\section{ÉTUDES SUR OU CONCERNANT GAO XINGJIAN}

ANTOMARINI Arunella:

- "La Peur de la beauté et la responsabilité esthétique », dans Autour de Gao Xingjian : éthique et esthétique pour aujourd'hui, Gémenos, Transbordeurs, 2003.

BÉJA Jean-Philippe:

- "Contre I"engagement" », dans La Quinzaine littéraire, 16-31 mars 2004.

BERGEZ Daniel :

- Gao Xingjian : peinture de l'âme, Paris, Seuil, 2013.

BOUVIER Béatrice:

- «L'Écriture de Gao Xingjian : fuite ou recherche des origines? », dans Mémoires, traces, récits: représentations et intertextualité, 2008, Cahiers du CIRHILL, Vol. 2, no 30, dirigé par Marie-Claude Rousseau, Paris, L'Harmattan, p. 65-84.

CHEN Shouzu (陈瘦竹) :

-〈论荒诞戏剧的衰落及其在我国的影响〉 《Lun huangdanxiju de shuailuo jiqi zai woguo de yingxiang » ("De la décadence du théâtre de l'absurde et de son influence dans notre pays»), dans 《社会科学评论》Shehui kexue pinglun ( La Critique des sciences humaines), 1985, no 11.

CHEN Xiaoming (陈晓明) :

- 〈从现代主义到没有主义〉 《Cong xiandaizhuyi dao meiyou zhuyi », dans 《二 十一世纪》Ershiyi shiji, 2000, no 62, p. 33-39. 


\section{Une subjectivité fluide}

COULTER Todd James:

- "The New Intellectual: Celebration of the Individual in the Plays of Gao Xingjian », dans The International Journal of the Humanities, vol. 5, 2007, no 5, p. 83-90.

CURIEN Annie:

- «Solitude et échos vocaux dans l'écriture de Gao Xingjian », dans L'Écriture romanesque et théâtrale de Gao Xingjian, Paris, Seuil, 2006, p. 169-187.

DAGEN Philippe:

- "L'Intérieur du regard», dans Gao Xingjian : encres, Paris, Galerie Claude Bernard, 2004, p. 5-9.

DRAGUET Michel :

- Le Goût de l'encre, Paris, Hazan, 2002.

- «Gao Xingjian ou la longue marche de l'encre », dans Gao Xingjian : encres, Paris, Galerie Claude Bernard, 2004, p. 11-15.

DU Gao (杜高), TANG Yin (唐因), ZHENG Bo'nong (郑伯农):

- 〈《车站》三人谈 〉 Chezhan sanrentan («L'entretien entre trois personnes à propos de L'Arrêt d'autobus »), dans 《戏剧报 » Xijubao (Le Journal du théâtre), 1984, no 3.

DUTRAIT Noël :

- «La Réception française de deux écrivains chinois contemporains: A Cheng et Gao Xingjian », dans France-Asie, un siècle d'échanges littéraires, Paris, You Feng, 2001, p. 153-160.

- «L'Itinéraire d'un homme seul », dans Esprit, décembre 2001, no 280, p. 146158.

- «L'Irrésistible poids du réel dans la fiction chinoise contemporaine : le cas de Gao Xingjian et de Mo Yan », dans Littérature chinoise, le passé et l'écriture contemporaine, Paris, La maison des sciences de l'homme, 2001, p. 35-44.

- "L'EFuvre de Gao Xingjian, un essai d'analyse globale», dans L'Écriture romanesque et théâtrale de Gao Xingjian, Paris, Seuil, 2006, p. 71-82.

- "Narration et sexe dans Le livre d'un homme seul de Gao Xingjian », actes du colloque "Traduire l'amour, la passion, le sexe dans les littératures extrêmeorientales », Aix-en-Provence, décembre 2006, publication en ligne : http://publications.univ-provence.fr/lct2006/index165.html

- 〈聆听高行健, 一个站在边缘的作家 〉 (Lingting Gao Xingjian, yige 'zhan zai bianyuan'de zuojia », dans 《联合报副刊》Lianhebao fukan, 25-26 juillet 2007.

- «Gao Xingjian de 'meiyou zhuyi': yigeren de zhuyi » « Le «sans-isme » de Gao Xingjian : le « isme » d'un homme seul], publié en chinois dans Hong Kong Drama Review, $n^{\circ} 8$, 2009, p. 53-61, en français dans Perspectives chinoises, février 2010, no 111, p. 8-14.

- "La Naissance d'un opéra d'expression chinoise: La Neige en août de Gao Xingjian », dans Perspectives chinoises, 2003, no 75, http://perspectiveschinoises. revues. org/60

FONG Gilbert C.F. (方梓勋) : 


\section{Bibliographie}

- Gao Xingjian - A Brief Chronology, Handout distributed at a seminar on the topic of « The Plays of Gao Xingjian », Hong Kong, International Association of Theatre Critics, 2002.

- 〈一双冷眼, 抽身观审 - 从高行健的创作论说起〉 Yishuang lengyan, choushen guanshen - Cong Gao Xingjian de chuangzuolun shuoqi, dans 《明报月刊: 高 行健特辑》Mingbao yuekan: Gao Xingjian teji, juin 2003, p. 44-45.

HU Yaoheng (胡耀恒) :

- 《百年耕耘的丰收——我对高行健戏剧的欣赏》(Bainian gengyun de fengshou - wo dui Gaong Xingjian xiju de xinshan), 台北 Taibei, 帝教出版社 Dijiao chubanshe, 1995.

HUANG Meixu (黄美序) :

-〈吒问高行健的《吒问死亡》：我的感觉与联想〉Kouwen Gao Xingjian de Kouwensiwang: wo de ganjue yu lianxiang, dans 《香港戏剧学刊》(Hong Kong Drama Review), 2009, no 8, p. 295-302.

JIN Siyan (金丝燕):

- "Dame-ivresse ou l'altérité inépuisable : le monde romanesque de Gao Xingjian », dans L'Ivresse dans tous ses états en littérature, Arras, Artois Presses Université, 2004, p. 289-311.

- "Littérature pure et exil littéraire », dans Exil à la frontière des langues (études réunies par Esther Heboyan-De Vries), Arras, Artois Presses Université, 2001, n 19, p. $31-57$.

KINKLEY Jeffrey C. :

- "Gao Xingjian in the "Chinese" perspective of Qu Yuan and Shen Congwen, dans Modern Chinese Literature and Culture, 2002, vol.14, no 2, p. 130-162.

ŁABEDZKA Izabella:

- Gao Xingjian's Idea of Theatre, From the Word to the Image, Leiden \& Boston, Brill, 2008.

LEE Mabel :

- "Gao Xingjian's Lingshan/Soul Mountain: Modernism and the Chinese Writer », dans HEAT, 1997, no 4, p. 128-143.

- "Pronouns as Protagonists: Gao Xingjian's Lingshan as Autobiography », dans China Studies, 1999, no 4, p. 165-183.

- «Pronouns as protagonists: on Gao Xingjian's théories of narration », dans Soul of Chaos, édité par Kwok-Kan Tam, Hong Kong, The Chinese University Press, 2001, p. 235-256.

- Aesthetic Dimensions of Gao Xingjian's Painting, Notre Dame, Snite Museum of Art, 2007.

LI Shixue (李通学):

- 〈高行健论中文〉Gao Xingjian lun zhongwen, dans 《中央日报》Zhongyang ribao, 30 nouvembre, 2000. 


\section{Une subjectivité fluide}

-〈朝圣心学 - 谈《灵山》〉Chaoshen xinxue - tan Lingshan, dans 《联合文 学》Lianhe wenxue, 2001, no 196.

LIU Zaifu (刘再复):

- "Afterword to On Man's Bible», dans Modern Chinese Literature and Culture, 2002, vol.14, no 2, p. 237-242.

- 《高行健论》Gao Xingjian lun, 台北 Taibei, 联经 Lianjing, 2004.

- 《高行健引论》Gao Xingjian yinlun, 香港 Hong Kong, 大山文化出版社 Dashan wenhua chubanshe, 2011.

MA Jian (马健) :

- 〈无限的遐想 - 高行健画中的悲凉与性意识〉Wuxian de xiaxiang - Gao Xingjian huazhong de beiliang yu xingyishi, dans 《明报月刊: 高行健特辑》 Mingbao yuekan: Gao Xingjian teji, juin 2003, p. 46-48.

- 〈重新开辟的语言境界 - 比较高行健和哈金的小说语言〉Chongxin kaipi de yuyan jingjie - bijiao Gao Xingjian he Hajin de xiaoshuo yuyan, dans 《华文文 学》Huawen wenxue, 2006, no 73.

QIAN Liqun (钱理群):

-〈现代汉语文学走过的路〉Xiandai hanyu wenxue zouguo de lu, dans 《二十一 世紀》Ershiyi shiji, 2000, no 62, p. 29-32.

QUAH Sy Ren :

- "Performance in Alienanted Voices: Mode of Narrative in Gao Xingjian's theatre », dans Modern Chinese Literature and Culture, 2002, vol.14, no 2, p. 51-98.

- Gao Xingjian and Transcultural Chinese Theater, Hawai'i, University of Hawai'i Press, 2004.

SU Chen (苏晨):

-〈高行健在花城起步〉 Gao Xingjian zai huacheng qibu, dans 《粤海风 》Yue Haifeng, 2008, no 6, P. 53-55.

TAM Kwok-Kan (谭国根):

- "Language as Subjectivity in One man's Bible», dans Soul of Chaos, édité par Kwok-Kan Tam, Hong Kong, The Chinese University of Hong Kong, 2001, p. 293310.

VEG Sébastian :

- «Un Signal d'alarme à Pékin vingt ans après », dans Vacarme, no 24, été 2003, p. 76-79.

- "De la marginalité à l'individualité dans les premières pièces de Gao Xingjian », dans L'Écriture romanesque et théâtrale de Gao Xingjian, Paris, Seuil, 2006, p. 147167.

- "Fuite sans fin et exil impossible: Le livre d'un homme seul de Gao Xingjian », dans Dans le dehors du monde: exils d'écrivains et d'artistes au xxe siècle, Paris, Presses Sorbonne nouvelle, 2010, p. 279-295.

XU Gang Gary : 
- «My writing, your pain, and her trauma: pronouns and (gendered) subjectivity in Gao Xingjian's Soul Moutain and One man's Bible », dans Modern Chinese Literature and Culture, 2002, vol.14, no 2, p. 99-129.

YANG Lian (杨炼) :

- 《逍遥如岛 - 高行健作品研究》, Xiaoyao ru niao - Gao Xingjian zuopin yanjiu, 台北 Taibei, 联经 Lianjing, 2012.

YANG Xiaobin (杨小滨):

-〈非政治的政治性: 高行健戏剧〉Fei zhengzhi de zhengzhixing: Gao Xingjian xiju, dans 《二十一世紀》Ershiyi shiji, 2000, no 62, p. 45-49.

YEUNG Jessica:

- Ink Dances in Limbo, Gao Xingjian's Writing as Cultural Translation, Hong Kong, The Hong Kong University, 2008.

ZHAO Yiheng (赵毅衡):

- 〈高行健在世界戏剧史的地位〉Gao Xingjian zai shijie xijushi de diwei, dans 《二十一世紀》Ershiyi shiji, 2000, no 62, p. 40-44.

- Towards A Merdern Zen Theatre - Gao Xingjian and Chinese Theatre Experimentalism, London, School of Oriental \& African Studies, 2000.

- 《 建立一种现代禅剧 - 高行健与中国实验戏剧》Jianli yizhong xiandai chanju - Gao Xingjian yu zhongguo shiyan xiju, 台北 Taibei, 尔雅出版社 Erya chubanshe, 2001.

- 〈欲界有禅 - 高行健笔下的男女肉体与精神关系〉 Yujie you chan - Gao Xingjian bixia de nannü routi yu jingshen guanxi, dans 《明报月刊: 高行健特 辑》Mingbao yuekan: Gao Xingjian teji, juin 2003, p. 57-62.

- "Posmodernité et zen dans les pièces récentes de Gao Xingjian », dans L'Écriture romanesque et théâtrale de Gao Xingjian, Paris, Seuil, 2006, p. 189-203.

ZHANG Xudong (张旭东):

- 〈承认的政治与被承认的期待〉 Chengren de zhengzhi yu beichengren de qidai, dans 《二十一世紀》Ershiyi shiji, 2000, no 62, p. 18-23.

ZHANG Yinde:

- "Littérature chinoise et comparatisme: l'exemple de Gao Xingjian», dans Littérature \& espaces, Actes du XXXe Congrès de la Société Française de Littérature Générale et Comparée, Limoges, Pulim, septembre 2001.

- "L'Esprit du paysage: Gao Xingjian et La Montagne de l'âme », dans Paysage \& modernité(s), Paris, Ousia, 2007, p. 293-311.

ZHU Dake (朱大可):

- 〈天鹅线审判和诺贝尔主义的终结〉 Tian'e'rong shenpan he nuobei'er zhuyi de zhongjie, dans 《二十一世紀》Ershiyi shiji, 2000, no 62, p. 24-28. 


\section{OUVRAGES GÉNÉRAUX EN LANGUE CHINOISE}

CAO Xueqin (曹雪芹) :

- 《脂砚斋重评石头记庚辰校本》Zhiyanzhai chongping shitouji gengchen jiaoben (Le Rêve dans le pavillon rouge), 北京Beijing, 作家出版社 Zuojia chubanshe, 2006.

CHEN Xiaoming (陈晓明) :

- 《中国当代文学主潮》Zhongguo dangdai wenxue zhuchao (Les mainstreams de la littérature chinoise contemporaine), 北京Beijing, 北京大学出版社Beijing daxue chubanshe, 2009.

CHEN Zizhan (陈子展) :

- 《诗经直解》Shijing zhijie, 上海 Shanghai, 复旦大学出版社 Fudan daxue chubanshe, 1983.

DU Xiaozhen (杜小真):

-〈法国哲学的魅力一从巴什拉《梦想的权利》谈起〉Faguozhexue de meili cong Bashila Meixiang de quanli tanqi, 《同济大学学报》（社科版）Tongji daxue xuebao, 2013, Vol. 24, no 1.

FENG Jicai (冯䩀才) :

-〈中国文学需要现代派〉 Zhngguo wenxue xuyao xiandaipai («La littérature chinoise a besoin de l'école moderne »), dans 《上海文学》Shanghai Wenxue (La littérature de Shanghai), 1982, no 8.

FENG Yidai (冯亦代) :

- 《这也是历史》Zhe yeshi lishi (《C'est aussi l'histoire»), dans 《读书》DuShu, 1983, no 7.

GAN Yang (dir.) (甘阳主编) :

- 《八十年代文化意识》Bashi niandai wenhua yishi, 上海Shanghai,上海人民出 版社Shanghai renmin chubanshe, 2006.

HAN Shaogong (韩少功) :

- 〈文学的根〉 Wenxue de gen ("Les racines de la littérature»), dans 《作家》 Zuojia (Ecrivains), 1985, nº 4.

- 《爸爸爸》Bababa, dans 《人民文学》Renmin wenxue, 1985, no 6.

HUANG Ziping (黄子平):

- 〈关于《伪现代派 》及其批评〉 Guanyu 'weixiandaipai'jiqi piping ("A propos de 'la fausse école moderne'et de ses critiques»), dans 《北京文学》Beijing wenxue, 1988, no 2.

HUI Neng (慧能) :

- 《坛经校释》Tanjing jiaoshi, 北京 Beijing, 中华书局 Zhonghua shuju, 1983.

JI Hongzhen (季红真) :

-〈中国近年小说与西方现代主义文学〉 Zhongguo jinnian xiaoshuo yu xifan xiandaizhuyi wenxue ("Les romans chinois récents et la littérature du modernisme occidental »), dans 《文艺报》Wenyibao, 2 janvier 1988. 


\section{Bibliographie}

JIANG Shaoyu (蒋绍愚)

- 《唐诗语言研究》Tangshi yuyan yanjiu, 郑州 Zhengzhou, 中州古籍出版社 Zhongzhou guji chubanshe, 1990.

KONG Zi (孔子):

-《四书章句集注・论语》Sishuzhangju jizhu - Lun yu，北京Beijing，中华书局 Zhonghua shuju, 2010.

KUBIN Wolfgang (顾涁) :

- 《二十世纪中国文学史》Ershi shiji zhongguo wenxueshi, (traduit de l'allemand en chinois), 上海 Shanghai, 华东师范大学 Huadong shifan daxue, 2008.

LAOZI (老子):

- 《老子道德经注校释 (王貆注)》Laozi Daodejing jiaozhu, 北京 Beijing, 中华书局 Zhnghua Shuju, 2008.

LEE Ou Fan (李欧梵) :

- 《铁屋中的呐喊》Tiewu zhong de nahan, 长沙 Changsha, 岳麓书社 Yuelu Shushe, 1999.

- 《未完成的现代性》Weiwancheng de xiandaixing, 北京Beijing, 北京大学出版社 Beijing daxue chubanshe, 2005.

LI Tuo (李陀) :

- 〈“现代小说” 不等于 “现代派” - 李陀给刘心武的信〉 'Xiandai xiaoshuo'budengyu 'xiandaipai' ("Le "roman moderne» ne s'identifie pas à l'« école moderne»), dans 《上海文学》Shanghai Wenxue (La littérature de Shanghai), 1982, no 8.

- 〈也谈《伪现代派》及其批评〉 Yetan 'weixiandaipai'jiqi piping ("Parlons 'du faux modernisme'et de ses critiques »), dans 《北京文学》Beijing wenxue, 1988, $n^{\circ} 4$.

LI Zehou (李泽厚):

- 《中国现代思想史论》Zhongguo xiangdai sixiangshi lu (De la pensée chinoise moderne), 北京 Beijing, 三联书店 Shanlian shudian, 2008.

LI Zehou (李泽厚) et LIU Zaifu (刘再复):

- 《告别革命, 李泽厚刘再复对话录》Gaobie geming, Li Zehou Liu Zaifu duihualu，香港 Hong Kong, 天地图书 Tiandi tushu， 2004.

LIANG Shuming (梁漱溟) :

- 《东西方文化及其哲学》Dongxifang wenhua jiqi zhexue, 上海Shanghai, 上海商 务印书馆Shanghai shangwu yinshuguan, 1922.

LIU Ban'nong (刘半农):

- 《刘半农诗选》Liu Ban'nong shixuan, 北京 Beijing, 人民文学出版社 Renmin wenxue chubanshe, 1956.

- 《刘半农文选》Liu Ban'nong wenxuan, 北京 Beijing, 人们文学出版社 Renmin wenxue chubanshe, 1986.

LIU Huairong (刘怀荣): 


\section{Une subjectivité fluide}

- 《赋比兴与中国诗学研究》Fubixing yu zhongguo shixue yanjiu, 北京 Beijing, 人 民出版社Renmin chubanshe, 2007.

LIU Xiaobo (刘晓波):

- 〈危机, 新时期文学面临危机〉Weiji, xinshiqi wenxue mianlin weiji ("Crise, la littérature de la nouvelle époque envisage la crise »), dans 《深圳青年报 》 Shenzhen qinnianbao (Journal de la jeunesse de Shenzhen), du 2 octobre 1986.

LIU Xicheng (刘锡诚) :

- 〈1982: 现代派的风波〉1982: Xiandaipai de fengbo («1982: la désaccord autour d'école moderne») dans 《南方文坛》Nanfang Wentang (Le Monde littéraire du Sud), 2014, no 1.

LIU Xie (刘劦思) :

- 《文心雕龙》Wenxin Diaolong, 上海 Shanghai, 上海古籍出版社Shanghai guji chubanshe, 2010.

- 《文心雕龙今释 (周振甫注释) 》Wenxin Diaolong Jinshi, 北京Beijing, 中华书 局 Zhonghua shuju, 2010.

LIU Xinwu (刘心武):

- 《班主任》Banzhuren (Enseignant de la classe), 北京Beijing, 中国青年出版社 Zhongguo qingnian chubanshe, 1979.

- 〈需要冷静地思考〉Xueyao lengjing de sikao («Nous avons besoin de réfléchir calmement »), dans 《上海文学》Shanghai Wenxue (La littérature de Shanghai), 1982, no 8.

LU Xinhua (卢新华) :

- 《伤痕》Shanghen (Cicatrices), dans 《文汇报》Wenhuibao, du 11 août 1978.

LU Xun (鲁迅):

- 《鲁迅全集》Luxun quanji (Euvres Complètes de Lu Xun), 20 volumes, 1 re édition en 1938, 上海 Shanghai, 光华书店 Guanghua Shudian, 2e en 1973, 北京 Beijing， 人民文学出版社 Renmin wenxue chubanshe:

- 〈文化偏至论〉 Wenhua pianzhi lun («Les aberrations de la culture»), dans 《坟》La Tombe, recueilli dans 《鲁迅全集》Luxun quanji (Euvres Complètes de LuXun), Vol. 1.

- 《过客》, Guoge (Le Passant), recueilli dans 《鲁迅全集》Luxun quanji (E Euvres Complètes de Lu Xun), Vol.1.

- 《影的告别》Ying de gaobie (Les adieux d'une ombre), recueilli dans 《鲁迅全集》 Luxun quanji (Euvres Complètes de Lu Xun), Vol.1.

- 《呐喊》Nahan (Cris), recueilli dans 《鲁迅全集》Luxun quanji (Euvres Complètes de Lu Xun), Vol.7.

- 《朝花夕拾》Zhaohua xishi (Fleurs du matin cueillies au soir), recueilli dans 《鲁迅全集》Luxun quanji (Fuvres Complètes de Lu Xun), Vol. 2.

- 《补天》Butian (Réparation du ciel), recueilli dans 《鲁迅全集》Luxun quanji (Euvres Complètes de Lu Xun), Vol.2.

- 〈论《第三种人»〉 Lun disanzhongren («De la 'troisième catégorie'»), recueilli dans 《鲁迅全集》Luxun quanji (Euvres Complètes de Lu Xun), Vol. 5. 
- 《中国小说史略》Zhongguo xiaoshuo shilue (L'Histoire du roman chinois), recueilli dans 《鲁迅全集》Luxun quanji (Euvres Complètes de Lu Xun), Vol. 9.

- 〈工人 绥惠略夫〉Gongren Shuihuiluefu (L'Ouvrier Shevyryov), recueilli dans《鲁 迅全集》Luxun quanji (Euvres Complètes de Lu Xun), Vol. 11.

MAO Zedong :

- 《伦理学原理批注》Lunlixue yuanli pizhu (Les Commentaires sur 'Du système de l'éthique), voir 《毛泽东早期文稿》Mao Zedong zaoqi wengao (Les Premiers écrits de Mao)，长沙 Changsha, 湖南人民出版社 Hunan renmin chubanshe, 1989.

-〈论新民主主义的民主和新民主主义的文化〉 Lun xinminzhuzhuyi de minzhu he xinminzhuzhuyi de wenhua («De la politique et la culture de la nouvelle démocratie»), dans 《中国文化》Zhongguo wenhua (La Culture chinoise), 1940, puis publié au titre〈新民主主义论〉Xinminzhuzhuyi lun («De la nouvelle démocratie ») dans 《解放》 Jiefang (La Libération), 1940, no 98-99.

MENG Zi (孟子):

-《四书章句集注・孟子》Sishuzhangju jizhu - Mengzi，北京Beijing，中华书局 Zhonghua shuju, 2010

MOU Zongsan (牟宗三) :

- 《中国哲学的特质》Zhongguo zhexue de tezhi, 上海 Shanghai, 上海古籍出版 社 Shanghai Guji Chubansh, 1997.

- 《中西哲学之会通十四讲》Zhongxi zhexue zhi huitong shisijiang,上海 Shanghai, 上海古籍出版社 Shanghai Guji Chubansh, 2007.

PEI Xiu (裴休):

- 《筠州黄藮山断际禅师传法心要》Junzhou huangboshan duanji chanshi chuangfa xinyao, 台北 Taibei, 国立师范大学出版社 Guoli shifan daxue chubanshe, 2012.

PLAKS Andrew H. :

- 《中国叙事学》Zhongguo xushixue (La Narratologie chinoise), C'est un recueil à la base des séminaires de Plaks donnés à l'université de Beijing en 1989, 北京 Beijing, 北京大学出版社Beijing Daxue Chubanshe, 1995.

PU Ji (普济) :

- 《五灯会元》 (全三册) Wudeng huiyuan (3 volumes), 北京Beijing, 中华书局 Zhonghua shuju, 1984.

QIAN Liqun (钱理群), HUANG Ziping (黄子平) et CHEN Pingyuan (陈平原) :

- 〈论二十世纪中国文学〉 Lun ershi shiji zhongguo wenxue («De la littérature chinoise du xxe siècle »), dans 《文学评论》Wenxue pinglun, (Critique de la littérature), 1985, no 5 .

- 《二十世纪中国文学三人谈》Ershi shiji zhongguo wenxue sanrentan (Parlons tous les trois de la littérature chinoise du xxe siècle), 北京Beijing, 人民文学出版 社 Renmin wenxue chubanshe, 1988.

QIAN Zhongshu (钱钟书) : 


\section{Une subjectivité fluide}

- 《管雉篇》(全五册), Guanzhui pian (5 volumes), 北京 Beijing, 中华书局 Zhonghua shuju, 1999.

QU Yuan (屈原):

- 《王注楚辞》Wangzhu chuci (Les Chants de Chu notés par Wang BI), Shanghai, Zhonghua Tushuguan (中华图书馆), 1917.

RONG Geng (容庚编著, 张振林, 马国权补编):

- 《金文编》Jinwen bian, 北京 Beijing, 中华书局 Zhonghua Shuju, 1985.

SHI Shengzhao (释僧肇):

- 《肇论校释》 Zhaolun jiaoshi, 北京Beijing, 中华书局 Zhonghua Shuju, 2010.

SHI Tao (石涛):

- 《苦瓜和尚画语录》Kugua heshang huayulu, 济南Jinan, 山东画报出版社 Shandong huabao chubanshe, 2007.

SIMA Xiangru (司马相如) :

- 《司马相如集校注》Sima Xiangru Jijiaozhu，上海 Shanghai，上海古籍出版社 Shanghai Guji Chubansh, 1993.

SIMA Qian (司马迁):

- 《史记》Shiji, 长沙 Changsha, 岳麓出版社 Yuelu chubanshe, 1988.

SU Shi (苏轼):

- 《苏轼文集》Sushi wenji, 北京 Beijing, 中华书局 Zhonghua shuju, 1986.

SUN Ge (孙歌) :

- 《主体弥散的空间-亚洲论述之两难》Zhuti misan de kongjian - Yazhou lunshu zhi liangnan, 南昌 Nanchang, 江西教育出版社 Jiangxi jiaoyu chubanshe, 2007.

TAKEUCHI Yoshimi (竹内好) :

- 《近代的超克》Jindai de chaoke, 北京Beijing, 三联书店 Shanlian Shudian, 2005.

- 《鲁迅》Lu Xun,杭州 Hangzhou, 浙江文艺出版社 Zhejiang Wenyi Chubanshe, 1986.

TAM Kwok-Kan (谭国根):

- 《主体建构政治与现代中国文学》(The Politics of Subject Construction in Modern Chinese Literature), Hong Kong, Oxford University press (China), 2000.

TAO YuanMing (陶渊明):

- 《陶渊明集箋注》Tao Yuanming jijianzhu, 北京 Beijing, 中华书局 Zhonghua shuju, 2011.

- 《搜神后记》Shoushen Houji, 上海 Shanghai, 上海古籍出版社 Shanghai guji chubansh, 2012.

TIAN Benxiang (田本相) :

- 《中国近现代戏剧史》Zhongguo jinxiandai xijushi, 南京 Nanjing, 江苏教育出版 社 Jiangsu jiaoyu chubansh, 2008.

WAN Hui (汪晖) :

- 《现代中国思想的兴起》Xiandai zhongguo sixiang de xingqi, 北京Beijing, 三联 Shanlian, 2004. 


\section{Bibliographie}

- 《反抗绝望》Fankang juewang, 北京Beijing, 三联书店 Shanlian Shudian, 2008 WANG Anyi (王安忆):

- 《小鲍庄》Xiaobaozhuang, dans 《中国作家》Zhouguo zuojia, 1985, no 2.

WANG Dewei (王德威):

- 《小说中国》Xiaoshuo Zhouguo, 台北 Taibei, 麦田出版社 Maitian chubanshe, 1993.

- 《写实主义小说的虚构: 茅盾, 老舍, 沈从文》Xieshizhuyi xiaoshuo de xugou: Maodun, Laoshe, Shencongwen, 上海 Shanghai, 复旦出版社 Fudan Chubanshe, 2001.

- 《被压抑的现代性:晚清小说新论》Bei yayi de xiandaixing: wanqing xiaoshuo xinlun, 北京Beijing, 北京大学出版社 Beijing daxue chubanshe, 2005.

WANG Fuzhi (王夫之):

- 《姜斋诗话笺注》Jiangzhai shihua jianzhu, 上海 Shanghai, 上海古籍出版社 Shanghai guji chubansh, 2012.

WANG Guowei (王国维):

- 《人间词话》Renjian cihua, 北京 Beijing，中华书局 Zhonghua shuju, 2011.

- 《宋元戏曲史》Songyuan xiqushi (L'Histoire du Xiqu des Songs et des Yuans), 北京 Beijing, 东方出版社 Dongfang chubanshe, 1996,

WANG Meng (王蒙):

- 〈王蒙给高行健的信〉Wangmen ge Gao Xingjian de xin, 《小说界》Xiaoshuo jie, 1982, no 2, p. 232 et p. 252.

- 《王蒙自传》Wangmeng zizhuang (L'Autobiographe de Wang Meng), 广州 Guangzhou, 花城出版社 Huacheng chubanshe, 2007.

WANG Tingxiang (王廷相) :

- 《王廷相集》Wangtingxiang ji, 北京 Beijing, 中华书局 Zhonghua shuju1989.

WANG Wei (王维):

- 《王维集》Wangwei ji, 北京 Beijing, 中华书局 Zhonghua shuju, 1997.

XIAO Gongqin (萧功秦):

- 《儒家文化的困境》Rujiawenhua de kunjing，成都 Chengdu，四川人民出版社 Sichuang renmin chubanshe, 1986.

XU Fuguan (徐复观) :

-〈释诗的比兴〉Shishi de bixing, dans 《民主评论》Minzhu pinglun, 1958, Vol. 9, no 15, recueilli dans 《中国文学精神》Zhongguo wenxue jingshen, 上海世纪出 版社 Shanghai Shiji chubanshe, 2006.

XU Shen (许慎) :

- 《说文解字》Shuowen jiezi, 北京 Beijing, 中华书局 Zhonghua shuju, 2002.

YANG Lian:

-〈幻想空间写作〉Huanxiang kongjian xiezuo, dans《沟通：面对世界的中国 文学》Goutong: miandui shijie de zhongguo wenxue, Stockholm, The Olof Palme International Centre, 1997. 


\section{Une subjectivité fluide}

- 《鬼话 - 智力的空间》Guihua·zhili de kongjian, 上海 Shanghai, 上海文艺出版社 Shanghaiwenyi chubanshe, 1998.

- 《大海停止之处》Dahai tingzhi zhichu,上海 Shanghai, 上海文艺出版社 Shanghaiwenyi chubanshe, 1998.

YE Lang (叶朗):

- 《中国美学史大纲》Zhongguo meixueshi dagang (Aperçu de l'histoire de l'esthétique chinoise), 上海人民出版社 Shanghai renmin zhubanshe, 1985.

YE Jiaying (叶嘉芗):

- 《迦陵论诗从稿》Jialing lunshi conggao, 石家庄 Shijiazhuang, 河北教育出版 社 Heibei jiaoyu chubanshe, 1998.

YE Xie (叶敛):

-〈原诗〉, Yuanshi, (收录于 《原诗, 一漂诗话, 说诗晬语》), 北京 Beijing, 人民 文学出版社 Renmin wenxue chubanche, 1979.

YIN Shun (印顺) :

- 《中国禅宗史》Zhongguo chanzongshi, 南昌 Nanchang, 江西人民出版社 Jiangxi renmin chubanshe, 2007.

YUAN Ke (袁珂):

- 《山海经校注》Shanhaijing jiaozhu (Les Notes du Classique des montagnes et des mers)，上海 Shanghai, 上海古籍出版社 Shanghai Guji Chubanshe, 1980.

YUNG Man-han (翁文㜀):

- «Observation of Postmodern Poetry in Taiwan: Writings of Hsia Yu and the New Generation (台湾后现代诗观察: 夏宇及其后的新一代书写)».Voir cet article entier sur site web : https ://docs. google. com/file/d/OB7qeU44v-c7wOWUydnVpR2RjR2s/edit.

ZHA Jianying (查建英) :

- 《八十年代访谈录》Bashi niandai fangtanlu, 北京Beijing, 三联书店 Shanlian shudian, 2006.

ZHANG Xudong (张旭东) :

- 《全球化时代的文化认同》Quangqiuhua shidai de wenhua rentong, 北京 Beijing, 北京大学出版社Beijing daxue chubanshe, 2009.

ZHAO Yihen (赵毅衡) :

- 〈“流外丧志”, 而后有文学: 关于海外大陆小说的几点观察〉 'Liuwai sangzhi', erhou you wenxue: guanyu haiwai dalu xiaoshuo de jidian guangcha, dans 《沟通: 面对世界的中国文学》Goutong: miandui shijie de zhongguo wenxue, Stockholm, The Olof Palme International Centre, 1997.

- 《意不尽言 -- 文学的形式-文化论》Yibujinyan - wenxue de xingshi-wenhualun, 南京Nanjing, 南京大学出版社 Nangjing daxue chubanshe, 2009.

- 《反讽时代 -- 形式论与文化批评》Fanfeng shidai - Xingshilun yu wenhuapiping, 上海 Shanghai, 复旦大学出版社 Fudan daxue chubanshe, 2011.

ZHONG Rong (钟嵘) : 
-《诗品释注》Shipin shizhu, 北京 Beijing, 中华书局 Zhonghua shuju, 1998.

ZHUANGZI (庄子):

- 《庄子集释》(全3册) Zhuangzi jishi (3 volumes), 北京 Beijing, 中华书局 Zhonghua shuju, 2004.

ZONG Baihua (宗白华):

- 《艺境》Yijing, 北京 Beijing, 北京大学出版社 Beijing Daxue Chubanshe, 1986.

ZUO Qiumin (左丘明) :

- 《左传》Zuozhuang, 长沙 Changsha, 岳麓出版社 Yuelu chubanshe, 1988.

\section{OUVRAGES GÉNÉRAUX EN LANGUES OCCIDENTALES (Y COMPRIS LES TRADUCTIONS)}

ABE Masao:

- Zen and Western Thought, London - Macmillan, University of Hawaii, 1985.

ADORNO Theodor W :

- Aesthetic Theory, London, Routledge \& Kegan Paul, 1984.

- Note to Literature, Columbia, Columbia University Press, Reprint édition, 1991.

ANDERSON Benedict:

- Imagined Communities: Reflections on the Origin and Spread of Nationalism, London, Verso, 1991.

ANDERSON Marston :

- The Limits of Realism: Chinese Fiction in the Revolutionary Period, Berkeley, University of California Press, 1990.

ARAGON Louis :

- Les Communistes, Paris, Le Livre de Poche, 1967.

ARISTOTE :

- Rhétorique (texte du Livres I et du Livre II établi et traduit par Médéric Dufour, texte du Livre III établi et traduit par Médéric Dufour et André Wartelle), Paris, Les Belles Lettres, 1980 pour le Livre III, 1990 pour le Livre I et le Livre II.

- Poétique (traduction inédite, présentation et notes de Michel Magnien), Paris, Le Livre de Poche, 1990.

- Les Métaphysiques (traduction analytique d'André de Muralt), Paris, Les Belles Lettres, 2010.

ARTAUD Antonin :

- Le Théâtre de la cruauté, dans La Nouvelle Revue française, 1932, no 229, p. 603614.

- Le Théâtre et son double, Paris, Gallimard, 1938.

BADIOU Alain :

- Circonstances 2, Irak, foulard, Allemagne/France, Clamecy, Léo Scheer, 2008. 


\section{Une subjectivité fluide}

BACHELARD Gaston :

- L'Eau et les rêves, Paris, José Corti, 1942.

BECKETT Samuel :

- Compagnie, Paris, Minuit, 1980.

- En attendant Godot, Paris, Minuit, 1952.

- L'Innommable, Paris, Minuit, 1953.

- Nouvelles et textes pour rien, Paris, Minuit, 1958.

- Proust, Paris, Minuit, 1990.

BENJAMIN Walter :

- Understanding Brecht, New Left Books, 1973.

- Essais sur Brecht (traduit de l'allemand par Philippe Ivernel), Paris, La Fabrique, 2003.

BENVENISTE Émile :

- Problèmes de linguistique générale I, Paris, Gallimard, 1966.

- Problèmes de linguistique générale II, Paris, Gallimard, 1974.

BERLIN Isaiah :

- Liberty, London, OUP Oxford, 2002.

BERMAN Marshall :

- All That is Solid Melts into Air -- The Experience of Modernity, New York, Penguin, 1982.

BLANCHOT Maurice:

- L'Espace littéraire, Paris, Gallimard, 1955.

BOOTH Wayne C :

- The Rhetoric of Fiction, Chicago \& London, The University of Chicago Press, 1961.

BRECHT Bertolt :

- Théâtre complet, Paris, L'Arche, 1975.

- Brecht on Theatre: the Development of an Aesthetic, edited and translated by John Willett, London, Eyre Methuen, 1964.

BROOK Peter :

- The Empty Space, UK, Penguin Classics, 1995.

- The Open Door: Thoughts on Acting and Theatre, US, Theatre Communications Group Inc., 1995.

- Entre deux silences, Arles, Actes sud-papiers, traduit de l'anglais par Marie-Hélène Estienne, 2006.

BUTOR Michel :

- La Modification, dans Fuvres complètes de Michel Butor, Paris, La Différence, 2010.

CALINESCU Matei :

- Five Faces of Modernity: Modernism, Avant-garde, Decadence, Kitsch, Postmodernism, Durham, Duke University Press, 1987.

CAMUS Albert: 
- Le Mythe de Sisyphe, Paris, Gallimard, 1942.

CAO Xueqin :

- Le Rêve dans le pavillon rouge (traduit par Li Tche-Houa et Jacqueline Alézaïs), Paris, Gallimard, 1981.

CHENG Anne:

- Histoire de la pensée chinoise, Paris, Seuil, 1997.

CHENG François :

- Vide et Plein : le langage pictural chinois, Paris, Seuil, 1979.

- L'Écriture poétique chinoise, Paris, Seuil, 1997.

CONFUCIUS:

- Les Entretiens de Confucius et de ses disciples (traduit du chinois par André Levy), Paris, Flammarion (GF), 1994.

- Entretiens de Confucius (traduit du chinois par Anne Cheng) Paris, Seuil, 1981.

COQUET Jean-Claude:

- «Temporalité et phénoménologie du langage, sur quelques problèmes posées par la phénoménologie à la linguistique et à la sémiotique », dans Sémiotique, décembre 1993, no 5, p. 9-29.

DE GOURMONT Remy:

- Le Livre des masques, Portraits symbolistes, Mercure de France, 1896.

DEMIÉVILLE Paul :

- «Le Miroir spirituel » dans Choix d'Études Bouddhiques (1929-1970), Leyde, Brill, 1973, p. 112-137.

DESCARTES :

- Méditations métaphysiques (Meditatio prima: texte latin accompagné de la traduction du duc de Luynes; Méditations de philosophie première: présentation et traduction de Michelle Beyssade), Paris, LGF, 1990.

DESCOMBES Vincent:

- Proust, philosophie du roman, Paris, Minuit, 1987.

DELEUZE Gilles :

- Proust et les signes, Paris, Puf, 2007.

DERRIDA Jacques:

- L'Écriture et la différence, Paris, Seuil, 1967.

- Marges de la philosophie, Paris, Minuit, 1972.

DUJARDIN Édouard:

- Les Lauriers sont coupés, Paris, Plon, 1973.

DUPONT Florence:

- Aristote ou le vampire du théâtre occidental, Paris, Flammarion, 2007.

DURAS Marguerite:

- Le Vice-consul, Paris, Gallimard, 1966.

- Détruire, dit-elle, Paris, Minuit, 1969. 


\section{Une subjectivité fluide}

- L'Amour, Paris, Gallimard, 1972.

DUTRAIT Noël :

- Littérature chinoise, état des lieux et mode d'emploi (Actes du colloque, sous la direction de Noël Dutrait), Publications de l'université de Provence, 1998.

- "La violence dans le roman chinois contemporain », dans Où va la Chine, Paris, Éditions du Félin, 2000, p. 172-190.

- Petit précis à l'usage de l'amateur de littérature chinoise contemporaine, Arles, Philippe Picquier, 2002.

EAGLETON Terry:

- The Illusions of Postmodernism, Oxford, Blackwell, 1997.

FREUD, Sigmund:

- Art and Literature, New York, Penguin, 1961.

- Inhibition, Puf (Quadrige), 1993.

FOUCAULT Michel :

- Les Mots et les choses, Paris, Gallimard, 1966.

- Dits et écrits II, Paris, Gallimard (Quarto), 2001.

FREDRIC Jameson:

- Postmodernism, or, the Cultural Logic of Late Capitalism, Durham, Duke University Press. 1991.

- «Reflection in Conclusion », dans Aesthetics and Politics, postfacé par Fredric Jameson, London, Verso, 2007.

GERNET Jacques :

- La Raison des choses, essai sur la philosophie de Wang Fuzhi (1619-1692), Paris, Gallimard, 2005.

GENET Jean :

- Querelle de Brest, Paris, Gallimard, 1953.

- Les Bonnes, Paris, Gallimard, 1978.

- Le Balcon, Paris, Folio, 1979.

- Splendid's, Paris, L'Arbalète, 1993.

- Elle, Paris, L'Arbalète, 1989.

GENETTE Gérard :

- Figures III, Paris, Seuil, 1972.

CHEN-ANDRO Chantal :

- "Les grands problèmes du roman en Chine au xxe siècle » dans Littératures d'Extrême-Orient au xx siècle, Arles, Philippe Picquier, 1993, p. 73-94.

HABERMAS, Jürgen :

- La Pensée postmétaphysique. Essais philosophiques, traduit de l'allemand par Rainer Rochlitz, Paris, Armand Colin, 1993.

- Le Discours philosophique de la modernité, traduit de l'allemand par Rainer Rochlitz et Christian Bouchindhomme, Paris, Gallimard, 1988. 


\section{Bibliographie}

HEGEL G. W. F. :

- Préface de la phénoménologie de l'esprit (traduction, présentation et vade-mecum par Jean-Pierre Lefebvre), Paris, Flammarion, 1991.

HSIA Chih-tsing :

- A History of Modern Chinese Fiction 1917-1957, New Haven, Yale University Press, 1961.

HUYSMANS Joris-Karl :

- À rebours, Paris, Georges Charpentier, 1884.

JIN Siyan :

- " Héritage : évolution ou révolution », dans Littérature chinoise : le passé et l'écriture contemporaine - regards croisés d'écrivains et de sinologues (sous la direction de Annie Curien et Jin Siyan), Paris, Maison des sciences de l'homme, 2001.

- "Dilemme identitaire», dans Neige d'août, Lyrisme et Extrême-Orient, Paris, automne, 2004, no 11, p. 50 - 71, et la suite dans la même revue, 2005, no 13, p. 30-40.

- "L'Écriture subjective dans la littérature chinoise contemporaine», dans Perspectives chinoises, 83 | Mai-juin 2004 : http://perspectiveschinoises.revues. org/1442.

- L'Écriture subjective dans la littérature chinoise contemporaine: devenir je, Paris, Maisonneuve \& Larose/Espace du temps présent, 2005.

JULLIEN François :

- Lu Xun, écriture et révolution, Paris, Presses de l'École normale supérieure, 1979.

- La Valeur allusive, des catégories originales de l'interprétation poétique dans la tradition chinoise, Paris, Puf (Quadrige), 2003.

- Le Détour et l'accès, Paris, Grasset, 1995.

- L'Écart et l'Entre. Leçon inaugurale de la chaire sur l'altérité, Paris, Galilée, 2012.

KUNDERA Milan :

- L'Art du roman, Paris, Gallimard, 1986.

LABORIT Henri :

- Éloge de la fuite, Paris, Gallimard, 2009

LACAN Jacques:

- "Fonction et champ de la parole et du langage en psychanalyse », dans La psychanalyse, 1956, no 1, p. 81-166.

- Les Quatre concepts fondamentaux de la psychanalyse, texte établi par JacquesAlain Miller, Paris, Seuil, 1973.

LAOZI:

- TaoTÖ King, le livre de la voie et de la vertu par Lao Tseu (traduction est empruntée à J. J. L. Duyvendak), Paris, Librairie d'Amérique \& d'Orient, 1953.

LEYS Simon:

- Essais sur la Chine, Paris, Robert Laffont, 1998. 


\section{Une subjectivité fluide}

LIANG Shuming :

- Les Cultures d'Orient et d'Occident et leurs philosophies (traduit du chinois par Luo Shenyi), Paris, Puf, 2000.

LIN Yü-Sheng :

- The Crisis of Chinese Consciousness, Madison Wi., The University of Wisconsin, 1979.

LIU He:

- Translingual Practice: Literature National Culture and Translated Modernity, China, 1900-1937, Stanford, Stanford University Press, 1995.

LU Xun :

- Lu Xun, Pamphlets et libelles (1925-1936) (présentation et traduction par Michelle Loi), Paris, François Maspero, 1977.

- "Les Aberrations de la culture » dans La Tombe (traduit par le groupe de traduction et d'étude de l'œuvre de Lu Xun constitué par Michelle Loi), Paris, Acropole, p. 65-86.

- Fleurs du matin cueillies au soir (traduit du chinoise), Beijing, Waiyu Chubanshe, 2004.

- « De la 'troisième catégorie'» (traduit par Michelle Loi), dans Lu Xun, Pamphlets et libelles (1925-1936), Paris, François Maspero, 1977.

- Le Passant (traduit par Pierre Ryckmans), dans La mauvaise Herbe, Bibliothèque Asiatique, Paris, 1975. p. 80-87.

- Les Adieux d'une ombre, (traduit par Pierre Ryckmans), dans La mauvaise Herbe, Bibliothèque Asiatique, Paris, 1975, p. 58-59.

- Cris (traduit par Joêl Bellassen, Feng Hanjin, Jean Join et Michelle Loi), Paris, Albin Michel, 1995.

- La Réparation du Ciel (traduit par François Jullien), dans Lu Xun, écriture et révolution, Paris, Presses de l'École normale supérieure, 1979.

MALRAUX André :

- Le Musée imaginaire, Paris, Gallimard, 1996.

- La Condition humaine, Paris, Gallimard, 1972.

MERLEAU-PONTY Maurice:

- Le Visible et I'Invisible, suivi de Notes de travail, Paris, Gallimard, 1964.

- Phénoménologie de la perception, dans Maurice Merleau-Ponty, Euvres, Gallimard (Quarto), 2010, p. 655-1167.

- L'EFil et l'esprit, dans Maurice Merleau-Ponty, Euvres, Gallimard (Quarto), 2010, p. 1547-1628.

- Le Doute de Cézanne, dans Maurice Merleau-Ponty, Fuvres, Gallimard (Quarto), 2010, p. 1306-1323.

- Visible et invisible, dans Maurice Merleau-Ponty, Euvres, Gallimard (Quarto), 2010, p. 1629- 1807.

- La Prose du monde, dans Maurice Merleau-Ponty, Euvres, Gallimard (Quarto), 2010, p. 1423-1543. 
- Sur Husserl, dans Maurice Merleau-Ponty, Euvres, Gallimard (Quarto), 2010, p. 1169-1290.

OWEN Stephen :

- Remembrances: the Experience of the Past in Classical Chinese Literature, Cambridge, Harvard University Press, 1986.

- Mi-Lou: Poetry and the Labyrinth of Desire, Cambridge, Harvard University Press, 1989.

PAVIS Patrice:

- Vers une théorie de la pratique théâtrale, Lille, Presses universitaires du Septentrion, 2007.

MICHAUX Henri :

- Un barbare en Asie, Paris, Gallimard, 1945.

- L'Espace du dedans, Paris, Gallimard, 1944.

NIETZSCHE Friedrich:

- Généalogie de la morale, Paris, Flammarion, 2000.

- Ainsi parlait Zarathoustra : un livre pour tous et pour personne (traduction, préface et commentaires de Georges-Arthur Galdschmidt), Paris, Le Livre de Poche, 1983.

PEREC Georges :

- La Disparition, Paris, Denoël, 1969.

- W ou le souvenir d'enfance, Paris, Denoël, 1975.

PIMPANEAU Jacques:

- Chine, histoire de la littérature, Arles, Philippe Picquier, 1989.

PLAKS Andrew $\mathrm{H}$. :

- «Towards a Critical Theory of Chinese Narrative», dans Chinese Narrative: Critical and Theoretical Essays, editor Andrew H. Plaks, New Jersey, Princeton University, 1977, p. 309-352.

PLATON :

- La République. Du régime politique (traduction de Pierre Pachet), Paris, Gallimard, 1993.

- L'Apologie de Socrate/Criton (traductions et introductions de Luc Brisson), Paris, Flammarion, 1997.

RABUT Isabelle:

- "L'Écriture romanesque de Shen Congwen, entre tradition et modernité », dans Littératures d'Extrême-Orient au xxe siècle, Arles, Philippe Picquier, 1993, p. 104124.

PRÉVERT Jacques:

- Paroles, Paris, Gallimard, 1949.

PROUST Marcel :

- Du côté de chez Swann, Paris, Flammarion, 1987.

- À l'ombre des jeunes filles en fleurs, Paris, Gallimard, 1988. 


\section{Une subjectivité fluide}

- Le Temps retrouvé, Paris, Gallimard, 1990.

RICCEUR Paul :

- Temps et récit 2: la configuration dans le récit de fiction, Paris, Seuil, 1984.

- La Métaphore vive, Paris, Seuil, 1975.

ROLAND Barthes:

- Carnets du voyage en Chine, Lonrai, Christian Bourgois, 2009.

SAINT AUGUSTIN :

- Les Confessions (traduction, préface et notes par Joseph Trabucco), Paris, Flammarion, 1964.

SAKAI Naoki :

- Voices of the Past: the Status of Language in Eighteenth-century Japanese Discourse, Ithaca, Cornell University Press, 1992.

SARTRE Jean-Paul :

- La Nausée, Paris, Gallimard, 1938.

- Qu'est-ce que la littérature, Paris, Gallimard, 1948.

SEGALEN Victor :

- René Leys, Paris, Gallimard, 1971.

STENDHAL:

- Chroniques italiennes, Paris, Gallimard, 1973.

- Souvenirs d'égotisme, Paris, Gallimard, 1983.

SU Shi :

- Su Dongpo sur moi-même (recueil des essais et poèmes de Su Shi, traduit du chinois et présenté par Jacques Pimpaneau), Arles, Picquier, 2003.

SUZUKI D.T., Erich Fromm et Richard de Martino:

- Bouddhisme Zen et psychanalyse, traduit par Théo Léger, Paris, Puf (Quadrige), 1971.

SZONDI Peter :

- Théorie du drame moderne (traduit de l'allemend par Sibylle Muller), Belval, Circé, 2006.

TAYLOR Charles:

- Sources of the Self, Cambridge, Harvard University Press, 1989.

TIAN MIN :

- The Poetics of Difference and Displacement: Twentieth-Century Chinese-Western Intercultural Theatre, Hong Kong, Hong kong University Press, 2008.

TOURAINE Alain :

- Critique de la modernité, Paris, Fayard, 1992.

VALÉRY Paul :

- «Aphorisme », dans La Nouvelle Revue française, septembre 1930, no 204, 


\section{Bibliographie}

VANDERMEERSCH Léon:

- Les Deux raisons de la pensée chinoise. Divination et idéographie, Paris, Gallimard, 2013.

WANG Wei :

- Paysages : Miroirs du cœur (recueil des poèmes de Wangwei, traduit du chinois en française par Wei-penn Chang et Lucien Drivod), Gallimard, Paris, 1990.

WEBER Max:

- Confucianisme et Taoïsme (traduit de l'allemand par Jean-Pierre Grossein et Catherine Colliot-Thélène), Paris, Gallimard, 2000.

YU Pauline:

- The Reading of Imagery in the Chinese Poetic Tradition, Princeton, Princeton University Press, 1987.

ZHANG Xudong:

- Chinese Modernism in the Era of Reforms, Durham, Duke University Press, 1997.

ZHANG Ning :

- L'Appropriation par la Chine du théâtre occidental: un autre sens de l'Occident, 19781989, Paris, L'Harmattan, 1998.

ZHANG Yinde :

- Le Monde romanesque chinois au xx siècle - modernités identités, Paris, Honoré, Champion, 2003.

- Littérature comparée et perspectives chinoises, Paris, L'Harmattan, 2008.

ZHUANGZI :

- Chuang-Tzu, the Inner Chapters (traduit du chinois en anglais par A. C. Graham), London, Boston, Allen \& Unwin, 1981.

- Fuvre complète de Tchouang-tseu, (traduit du chinois en français par Liou Kiahway), Paris, Gallimard, 1969.

- Les Fuvres de maître Tchouang (traduit du chinois en français par Jean Levi), Paris, L'Encyclopédie des Nuisances, 2006.

ZIMA Pierre V.

- La Négation esthétique : le sujet, le beau et le sublime de Mallarmé et Valéry à Adorno et Lyotard, Paris, L'Harmattan, 2002. 



\section{Table des matières}

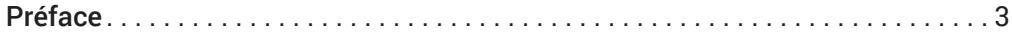

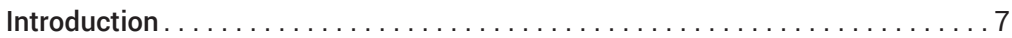

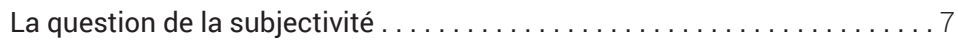

La modernité et la modernisation : l'individu et le collectif . . . . . . . . 10

De qui suis-je ? à où suis-je ?: le sujet percevant a-ontologique. . . . . . . . 13

\section{PREMIÈRE PARTIE}

\section{La modernité ambiguë: comment la subjectivité s'implante dans}

la littérature chinoise moderne. . . . . . . . . . . . . . . . . . 19

1. Y a-t-il du modernisme en Chine au début des années 1980 ? . . . . . . . . 19

L'école moderne .................................. 19

Entre « école moderne » et réalisme : ambiguïtés . . . . . . . . . . . 19

La recherche de la forme . . . . . . . . . . . . . . . . . . 26

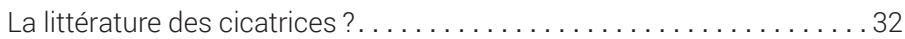

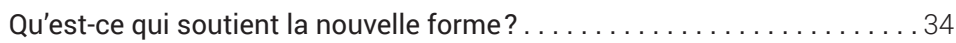

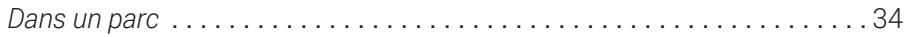

L'innovation de la forme et la réforme de la société . . . . . . . . . . 38

Qu'est-ce que la modernité chinoise $? \ldots \ldots \ldots \ldots \ldots \ldots \ldots \ldots \ldots \ldots 41$

Un modernisme retardé $? \ldots \ldots \ldots \ldots \ldots \ldots \ldots \ldots \ldots \ldots \ldots \ldots \ldots$

L'individualisme au début des années 1980 interprété par Li Zehou . . . 45

Le moi submergé et le moi expansif. ..................48

La littérature ne peut être que la voix d'un individu ............ 53

Auteur apolitique et politisé, moderne et héritant de la tradition . . . . . . 59

Le paradoxe du moi chez Gao . . . . . . . . . . . . . . . . . . . 61

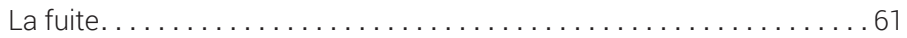

Entre le moi chaos et le moi qui ne s'appuie ni sur le

subjectivisme ni sur l'objectivisme ...................... 66

Le non-isme et le paradoxe de la liberté de l'individu . . . . . . . . . 70

2. Autour des notions de temps et d'ombre de la subjectivité . . . . . . . . 75

Les concepts de sujet et de temps dans L'Arrêt d'autobus

et En attendant Godot . . . . . . . . . . . . . . . . . . . . . . . . . . . . . . 75

Un présent éternel et un présent absent . . . . . . . . . . . . . 75

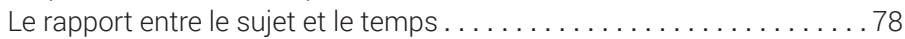

La montre et les temps modernes $\ldots \ldots \ldots \ldots \ldots \ldots \ldots \ldots \ldots$ 
De Lu Xun à Gao Xingjian: la bifurcation de la subjectivité. . . . . . . . 89

L'Arrêt d'autobus et Le Passant . . . . . . . . . . . . . . . . . . . . 89

L'ombre : le jeu entre la politique et la subjectivité..............93

La figure de Nü Wa ............................. 109

\section{DEUXIÈME PARTIE}

\section{Y a-t-il une subjectivité fluide?}

3. La narratologie des pronoms personnels et le sujet percevant . . . . . . . 117

La perception d'un « autre moi »........................ 117

Les pronoms personnels comme une manière de percevoir . . . . . . . 117

L'autre moi à percevoir . . . . . . . . . . . . . . . . . . . . . . . . . . 124

La fluidité de la perception : révélatrice du désir . . . . . . . . . . 129

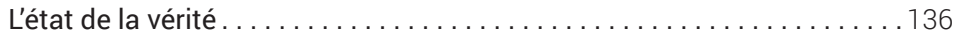

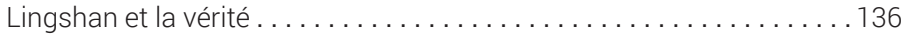

La vérité - zhenshi .......................... 140

Qui cherche et éprouve la vérité ? . . . . . . . . . . . . . . . . 144

Un autre mode du percevoir : I'« entre » (间) . . . . . . . . . . . . . . . 147

Comment percevoir le zhenshi et l'acteur neutre . . . . . . . . . . . . 147

$L^{\prime}$ « entre» (间) comme un passage. . . . . . . . . . . . . . . 153

4. Mon regard n'est pas en moi . . . . . . . . . . . . . . . . . . . . . . . . . . . . . 161

Mon regard est en dehors de mon corps . . . . . . . . . . . . . . 161

Le corps et l'œil. . . . . . . . . . . . . . . . . . . . . . . . . . . . . . 161

L'invisible du visible ............................ 167

Miroir transparent de l'intérieur. . . . . . . . . . . . . . . 173

Une autre manière de regarder le moi en rapport avec la pensée du

bouddhisme chán ...................................179

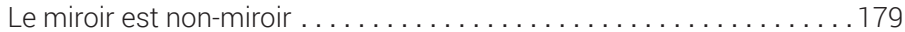

Se perdre pour atteindre le vrai moi ...................... 184

Je me perds dans la vision où je suis, je me « retrouve » dans la

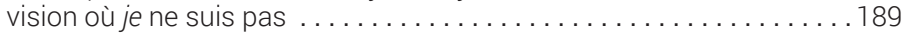

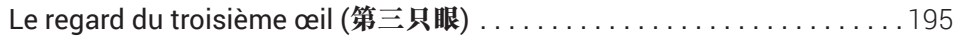

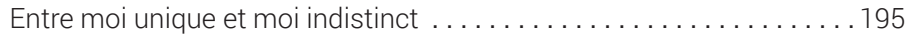

Un regard froid du moi sur moi-même sans rapport à la subjectivité . .200

L'extension entre le monde extérieur et le monde intérieur : le paysage 206

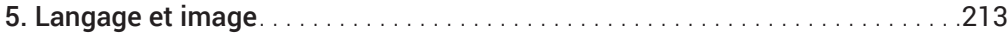

La subjectivité émerge-t-elle dans le langage $\ldots \ldots \ldots \ldots \ldots \ldots \ldots 213$

Pourquoi le courant de langage au lieu du courant de conscience? . . . 213

Une autre «logique » pour employer les pronoms personnels :

comment la subjectivité fluide émerge dans le langage . . . . . . . . . 224

Premier acte de Dialoguer-interloquer : le langage à la fois fermé et

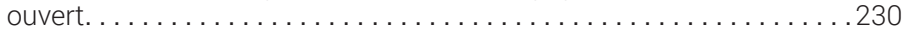

Deuxième acte de Dialoguer-interloquer: où suis-je lorsque je parle?. . . 238

Image et langage: ni subjectivé ni objectivé..................245

Qu'est-ce que le langage évocateur (唤起的语言) ? Le langage

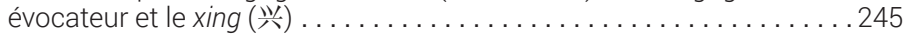

La réversibilité entre le moi et le monde et entre le moi intérieur et 
le moi extérieur . . . . . . . . . . . . . . . . . . . . . . . . 251

Le yixiang (意象) est un processus . . . . . . . . . . . . . . 259

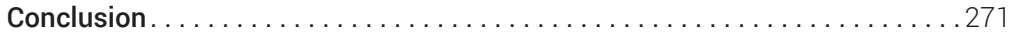

La subjectivité fluide dans la transculture $\ldots \ldots \ldots \ldots \ldots \ldots \ldots \ldots 271$

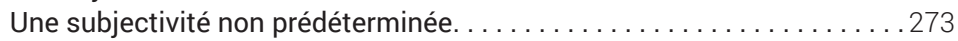

L'Errance de l'oiseau. . . . . . . . . . . . . . . . . . . . . . . . 276

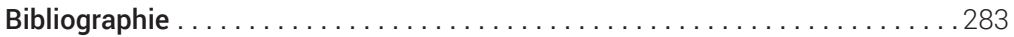

Ouvrages de Gao Xingjian . . . . . . . . . . . . . . . . . . . . . . . 283

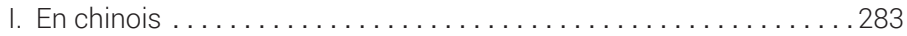

II. En Français et en anglais (y compris les traductions du chinois) . . .286

Études sur ou concernant Gao Xingjian. . . . . . . . . . . . . . . . . 289

Ouvrages généraux en langue chinoise . . . . . . . . . . . . . . . . . . 294

Ouvrages généraux en langues occidentales (y compris les traductions) 301 


\section{Déjà parus aux éditions DEMOPOLIS}

\begin{tabular}{|c|c|}
\hline $\begin{array}{l}\text { Brossat, Alain } \\
\text { Abécédaire Foucault }\end{array}$ & $\begin{array}{l}\text { Garo, Isabelle } \\
\text { Foucault, Deleuze, Althusser et Marx, Karl }\end{array}$ \\
\hline $\begin{array}{l}\text { Boltanski, Luc } \\
\text { Rendre la réalité inacceptable }\end{array}$ & $\begin{array}{l}\text { Goldberg, Jeffrey } \\
\text { Prisonnier en terre promise }\end{array}$ \\
\hline $\begin{array}{l}\text { Bourdieu, Pierre - Boltanski, Luc } \\
\text { La Production de l'idéologie dominante }\end{array}$ & $\begin{array}{l}\text { Guérin, Isabelle } \\
\text { La microfinance et ses dérives }\end{array}$ \\
\hline $\begin{array}{l}\text { Césaire, Aimé - Malcolm X } \\
\text { Black revolution }\end{array}$ & $\begin{array}{l}\text { Halliday, fred } \\
100 \text { idées reçues sur le Moyen-Orient }\end{array}$ \\
\hline $\begin{array}{l}\text { Chuzeville, François } \\
\text { Zimmerwald }\end{array}$ & $\begin{array}{l}\text { Hobsbawm, éric } \\
\text { Marx et l'histoire }\end{array}$ \\
\hline $\begin{array}{l}\text { Clover, Charles } \\
\text { Surpêche }\end{array}$ & $\begin{array}{l}\text { Hroub, Khaled } \\
\text { Le Hamas }\end{array}$ \\
\hline $\begin{array}{l}\text { Cohen-Seat, Patrice } \\
\text { Peuple! Les luttes de classes au xxie siècle }\end{array}$ & $\begin{array}{l}\text { Jennar, Raoul Marc } \\
\text { Khieu Samphan \& les Khmers rouges }\end{array}$ \\
\hline $\begin{array}{l}\text { Da Lage, Olivier (sous la dir. de) } \\
\text { Qatar: les nouveaux maîtres du jeu }\end{array}$ & $\begin{array}{l}\text { Khaldi, Eddy } \\
\text { Abc de la laïcité }\end{array}$ \\
\hline $\begin{array}{l}\text { Denord, François } \\
\text { Néo-libéralisme, version française } \\
\text { Dhume-Sonzoani. Fabrice }\end{array}$ & $\begin{array}{l}\text { Kautsky, Karl } \\
\text { Les luttes de classe pendant la Révolution } \\
\text { française }\end{array}$ \\
\hline $\begin{array}{l}\text { Communautarisme. Enquête sur une } \\
\text { chimère du nationalisme français }\end{array}$ & $\begin{array}{l}\text { Khaldy, Eddy - Fitoussi, Muriel } \\
\text { Main basse sur l'École publique }\end{array}$ \\
\hline $\begin{array}{l}\text { Duclert, Vincent } \\
\text { Occupy Gezi }\end{array}$ & $\begin{array}{l}\text { La République contre son école } \\
\text { Kalfon, Pierre }\end{array}$ \\
\hline $\begin{array}{l}\text { Durpaire, François - } \\
\text { Richomme, Olivier } \\
\text { L'Amérique de Barack Obama } \\
\text { Obama face à la crise }\end{array}$ & $\begin{array}{l}\text { Chroniques chiliennes } \\
\text { Kamata, Satoshi, } \\
\text { Toyota: I'usine du désespoir } \\
\text { Landriève, Sylvie }\end{array}$ \\
\hline $\begin{array}{l}\text { Ferrette, François } \\
\text { La véritable histoire du Parti communiste } \\
\text { français }\end{array}$ & $\begin{array}{l}\text { L'immobilier. Une passion française } \\
\text { Loubes, Olivier } \\
\text { Jean Zay. Réarmer la République }\end{array}$ \\
\hline $\begin{array}{l}\text { Gaulard, Mylène } \\
\text { Karl Max à Pékin - Les racines de la crise en } \\
\text { Chine capitaliste }\end{array}$ & $\begin{array}{l}\text { Mamdani, Mahmoud } \\
\text { La ClA et la fabrique } \\
\text { du terrorisme islamique }\end{array}$ \\
\hline
\end{tabular}




\author{
Mendes France, Pierre \\ Liberté Liberté chérie \\ Nadaud, Stéphane \\ Les Japons de Kenzaburo Oé \\ Labat, Séverine \\ Les islamistes tunisiens - entre l'état et la \\ mosquée \\ Latour, Bruno - Lippman, Walter \\ Le public fantôme \\ Lénine \\ Petit manuel pour rompre avec le \\ capitalisme \\ 1914, repenser le nationalisme et la guerre \\ Mandani, Mahmood \\ La CIA et la fabrique du terrosisme islamiste \\ Marx, Karl \\ Qu'est-ce que le capitalisme? \\ Les Crises du capitalisme \\ Le Capital financier \\ Mordillat, Gérard - \\ Prieur, Jérôme \\ De la crucifixion considérée \\ comme un accident du travail \\ Gérard Mordillat présente \\ « Le fascisme » de Mussolini \\ Nsar, Vali \\ Le renouveau chiite \\ Pivert, Marceau \\ Une histoire populaire de la laïcité \\ Prochasson, Christophe \\ L'Empire des émotions : \\ les historiens dans la mêlée
}

Rebérioux, Madeleine

Vive la République

Rodinson, Maxime

Islam et capitalisme

Sassen, Saskia

Critique de l'État

Saurin, Patrick

Les prêts toxiques: une affaire d'état

Shah, Sonia

Cobayes humains : le grand secret des

essais pharmaceutiques

Uchitelle, Louis

Le salarié jetable

Wallerstein, Immanuel

L'Universalisme européen :

de la colonisation au droit d'ingérence

Wilkinson, Richard

L'égalité, c'est la santé

Winder, Simon

James Bond

Walt, Stephen

Pourquoi le monde n'aime plus les états-Unis

Whitaker, Brian

Parias : Gays et Lesbiennes dans le monde arabe 


\section{Collection « Quaero »}

Cassin, Barbara et Wosny, Danièle (dir.) Les intraduisibles du patrimoine en Afrique subsaharienne

Dawod, Hosham (dir.) La constante "Tribu », variations arabomusulmanes

Cassin, Barbara et Gorog, Françoise Psychanalyser en langues. Intraduisibles et langue chinoise

Ehrenfreund, Christian et Schreiber, JeanPhilippe (dir.)

Les marranismes. De la religiosité cachée à la société ouverte

Ethis, Emmanuel

Le cinéma près de la vie

Fernadez Garcia, Alicia et Petithomme, Mathieu, Contester en Espagne

Fontaine, Alexandre

Aux heures suisses de l'école républicaine

Ghasarian, Christian

Rapa. Île du bout du monde, île dans le monde

Ghosn, Katia

Rachid El-Daiif. Le roman arabe dans la tourmente de la modernsisation

Aubert-Nguyen, Hoai Huong et Espagne, Michel (dir.)

Le Vietnam. Une histoire de transferts

culturels

Niveleau, Charles-édouard (dir.)

Vers une philosophie scientifique - Le programme de Brentano

Oléron Evans, Emilie

Nikaulos Pevsner. Arpenteur des arts

Rabault-Feuerhahn,

Pascale (dir.)

Théorie intercontinentales - Voyages du comparatisme postcolonial

Salamagne, Michèle- $\mathrm{H}$. et Thominet, Patrick (dir.),

Accompagner. Trente ans de soins palliatifs en France

\section{Collection Philosophie en cours}

Cavaillé, Christian

Les jeux de langage chez Wittgenstein

Chauve, Alain

Le Tractacus : logique et métaphysique

Sfez, Gérald

Machiavel et la vérité politique

Tomès, Arnaud

Castoriadis. L'imaginaire, le rationnel et le réel 

Achevé d'imprimer en France en 2017 dans les ateliers de Dupli-print à Domont (95)

$N^{\circ}$ d'impression : 2017021701

Dépôt légal : mars 2017 